\title{
AIR PARTICULATE MATTER IN POLLUTED \\ NEW ZEALAND URBAN ENVIRONMENTS: SOURCES, PATTERNS AND TRANSPORT
}

BY

\section{TRAVIS ANCELET}

\author{
A thesis \\ submitted to the Victoria University of Wellington \\ in fulfillment of the requirements for the degree of \\ Doctor of Philosophy
}

Victoria University of Wellington

2012 


\section{Abstract}

During the winters of 2010 and 2011, three intensive particulate matter (PM) monitoring campaigns were undertaken in Masterton, Alexandra and Nelson, New Zealand. The goal of these campaigns was, for the first time, to identify the sources and factors contributing to elevated PM concentrations on an hourly time-scale. In each location, hourly coarse ( $\mathrm{PM}_{10-2.5}$; particles with aerodynamic diameters $2.5 \mu \mathrm{m}<$ $d<10 \mu \mathrm{m})$ and fine $\left(\mathrm{PM}_{2.5}\right.$; particles with aerodynamic diameters $\left.<2.5 \mu \mathrm{m}\right)$ samples, $\mathrm{PM}_{10}$ (particles with aerodynamic diameters $<10 \mu \mathrm{m}$, incorporating the coarse and fine fractions) concentrations and meteorological variables were collected from a number of sites. Using elemental concentrations determined from ion beam analysis and black carbon concentrations determined from light reflection for each hourly sample, PM sources and their contributions on an hourly time-scale were identified using positive matrix factorization (PMF).

In Masterton, where two sampling sites were employed, $\mathrm{PM}_{10}$ concentrations displayed distinct diurnal cycles, with peak concentrations occurring in the evening (7 pm-midnight) and in the morning (7-9 am). Four PM sources were identified (biomass burning, marine aerosol, crustal matter and vehicles) at each of the sites and biomass burning was identified as the most dominant source of $\mathrm{PM}_{10}$ during both the evening and morning. One of the sites experienced consistently higher $\mathrm{PM}_{10}$ concentrations and katabatic flows across Masterton were identified to be the main contributor to this phenomenon.

In Alexandra and Nelson, three sampling sites on a horizontal transect (upwind, central and downwind of the general katabatic flow pathway) and a fourth site located centrally, but at a height of $26 \mathrm{~m}$, were incorporated in a novel study design. Each of the sites in Alexandra and Nelson also showed diurnal patterns in $\mathrm{PM}_{10}$ concentrations. The central site in Alexandra experienced consistently higher $\mathrm{PM}_{10}$ concentrations and four $\mathrm{PM}_{10}$ sources were identified at each of the sites (biomass burning, marine aerosol, vehicles and crustal matter). Biomass burning was identified as the main source of $\mathrm{PM}_{10}$ throughout the day at each of the sites. The convergence of numerous katabatic flows was identified as the contributing factor to the elevated $\mathrm{PM}_{10}$ concentrations measured at the central site. 
In Nelson, five PM sources were identified at each of the sites (biomass burning, vehicles, marine aerosol, shipping sulfate and crustal matter) and biomass burning was identified as the dominant source of $\mathrm{PM}_{10}$ throughout the day. Katabatic flows were also identified to play an important role in $\mathrm{PM}_{10}$ transport.

Analyses of source-specific (wood combustion and vehicles) PM samples was also undertaken, and the results of these analyses are included in this thesis. 


\section{Acknowledgements}

I am deeply appreciative to my supervisor at GNS Science, Dr. Perry Davy, for allowing me to be part of this exciting research program. Perry has taught me much of what I now know and his door was never closed, even when that door was at his home. Perry was also extremely flexible, providing me the freedom to pursue my interests, encouraging me to attend each and every conference that I could and even allowing me to bring my dog, Linus, with me to the South Island while the research program was in full-swing. I feel extremely fortunate to have worked for you.

I also appreciate the support of my supervisor at Victoria University of Wellington, Dr. David Weatherburn. David was always there to provide me with support, even in retirement. Thanks for all the helpful comments on manuscripts and for organizing anything I needed from the university.

Drs. Bill Trompetter and Andreas Markwitz are thanked for their support and insight. They were always more than happy to discuss anything, science-related or not. Bill, in particular, has taught me much about ion beam analysis and I am extremely grateful for that.

It would not have been possible to complete this thesis without the love and support of many individuals. My parents have always supported me, providing me with every opportunity to succeed, even if I was all the way around the world. Thank you for believing that I could achieve anything I put my mind to. I also want to thank my Papa, I know how hard it has been for me to be so far from home, but you have always supported me.

Last, but certainly not least, my wife Lindsay has been a fundamental pillar in my life. Thank you for being the most amazing woman I know, allowing me to leave for months at a time to collect samples or attend conferences. This journey has been as much yours as it has been mine and I want to thank you for your love, patience and all the beautiful things that you are. 


\section{Table of Contents}

Abstract.................................................................................................................................

Acknowledgements ....................................................................................................................... iii

Table of Figures............................................................................................................................ viii

Table of Tables .......................................................................................................................... xiii

List of Abbreviations ...............................................................................................................

1. Atmospheric Aerosols - An Introduction .....................................................1

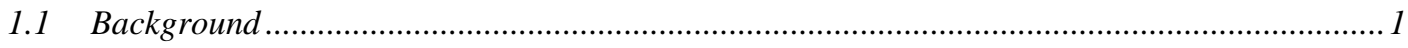

1.2 General Characteristics of Atmospheric Aerosols ...........................................................1

1.2.1 Size Distribution of Atmospheric Aerosol Particles ...........................................................2

1.2.2 Sources and Composition of Atmospheric Aerosol Particles ............................................. 4

1.2.3 Particle Lifetimes in the Atmosphere ...........................................................................

$1.3 \quad$ Health Effects of Atmospheric Aerosols ......................................................................

1.4 Environmental and Climate Effects of Atmospheric Aerosols.................................................6

2. Atmospheric Aerosols - Analysis ............................................................................10

2.1 Collection of Atmospheric Aerosols ...................................................................................10

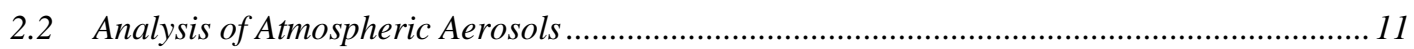

2.2.1 Atomic Absorption Spectroscopy (AAS) …………………………………………....... 12

2.2.2 Inductively Coupled Plasma - Atomic Emission Spectroscopy (ICP-AES) and Mass

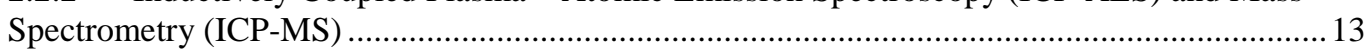

2.2.3 X-ray Fluorescence (XRF) ……………………………………………………... 13

2.2.4 Ion Beam Analysis (IBA) ……………………….................................................... 14

2.2.5 Instrumental Neutron Activation Analysis (INAA) ..................................................... 14

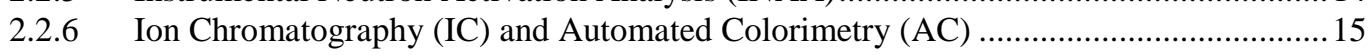

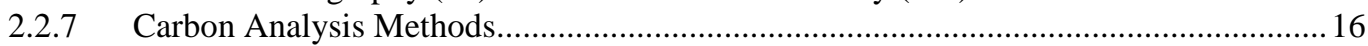

2.2.8 Scanning Electron Microscopy - Energy Dispersive Spectroscopy (SEM-EDS) ............20

3. Atmospheric Aerosols - Source Apportionment...................................................21

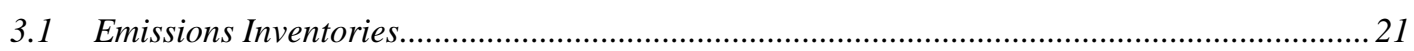

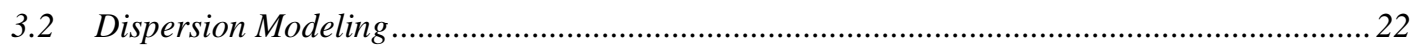

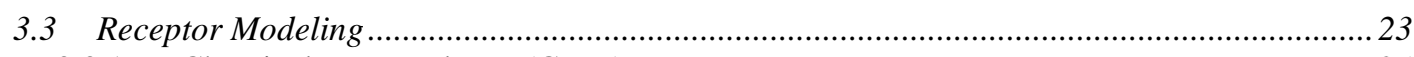

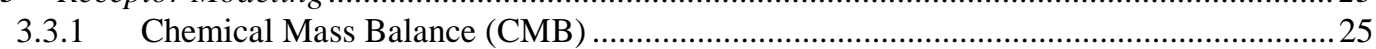

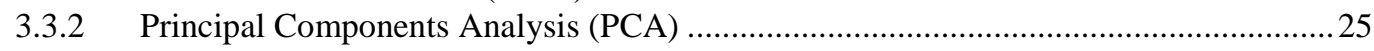

3.3.3 Positive Matrix Factorization (PMF) ..........................................................................2 26

4. A review of air particulate matter research in New Zealand: Where are we and where should we go from here?..............................................................................2

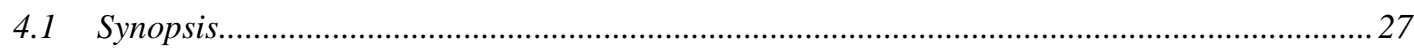

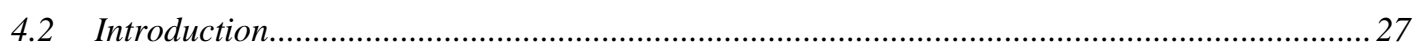

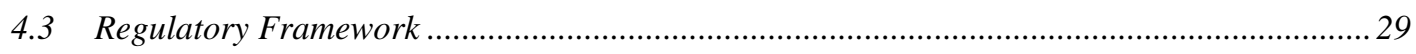

$4.4 \quad P M_{10}$ Concentrations Throughout New Zealand ...................................................................... 30

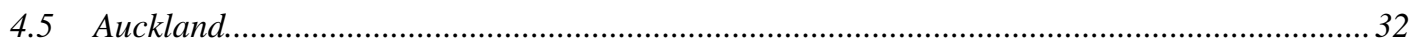




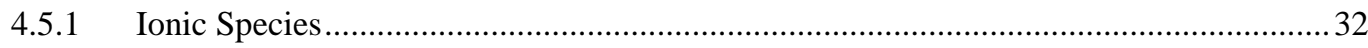

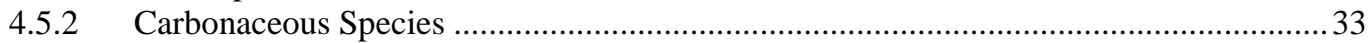

4.5.3 Source Apportionment Studies ................................................................................. 34

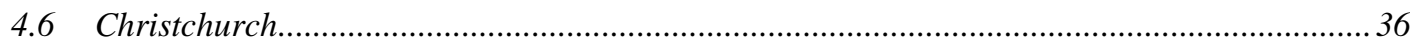

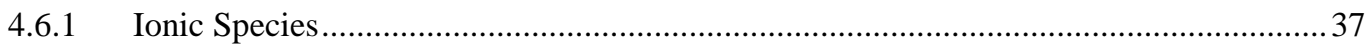

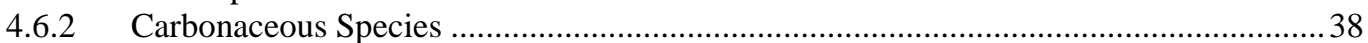

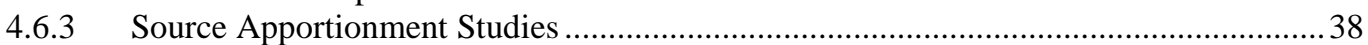

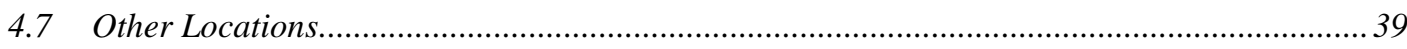

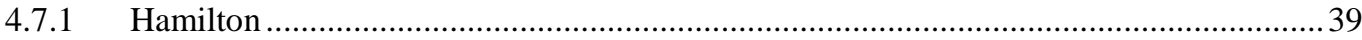

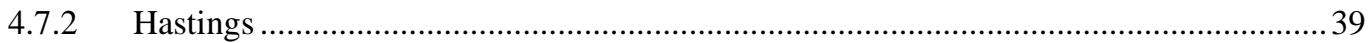

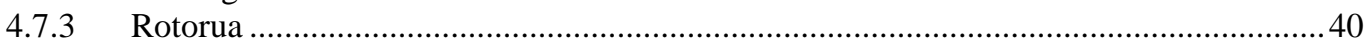

4.7.4 Greater Wellington Region.................................................................................. 40

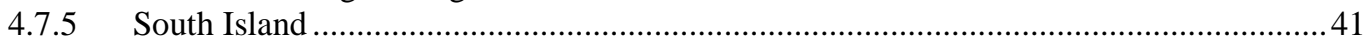

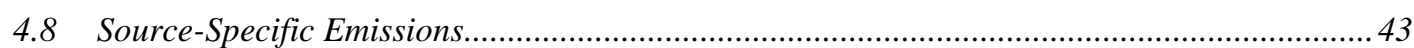

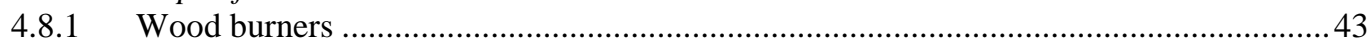

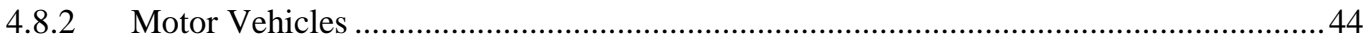

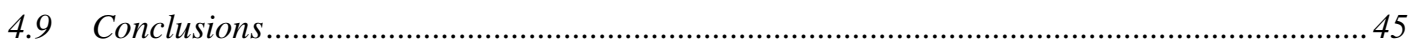

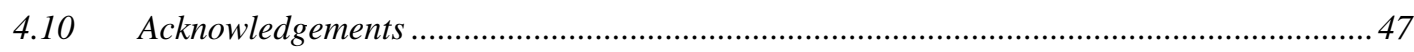

5. Research objectives...............................................................................................48

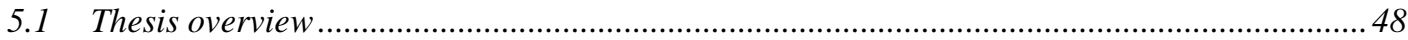

6. A comparison of particulate and particle-phase PAH emissions from a modern wood burner with those of an old wood burner........................................50

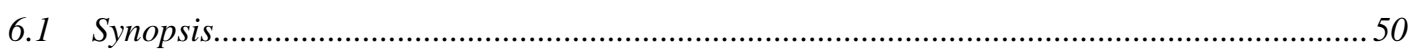

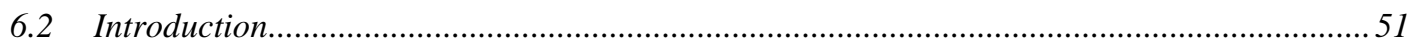

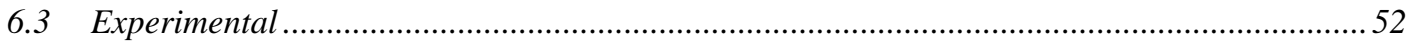

6.3.1 Wood burner emissions sampling .......................................................................... 52

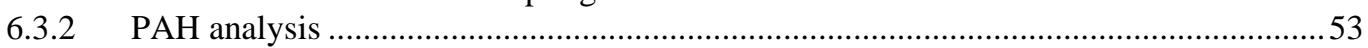

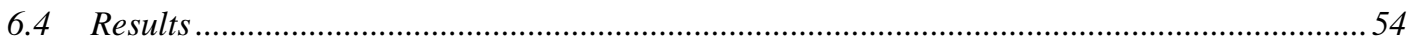

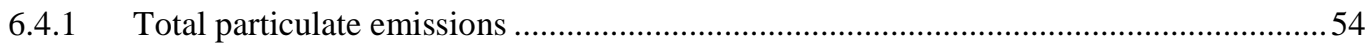

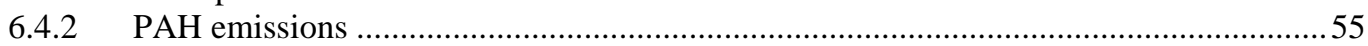

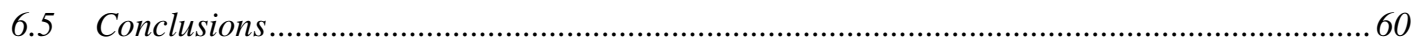

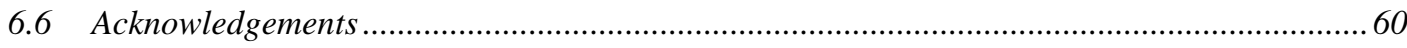

\section{Characterisation of particulate matter emissions from a modern wood} burner under varying burner conditions..................................................................661

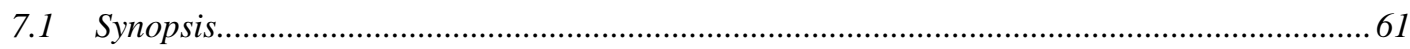

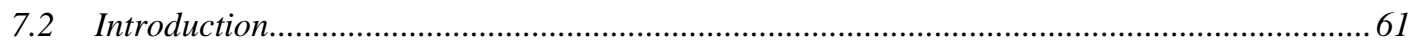

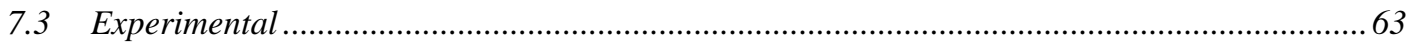

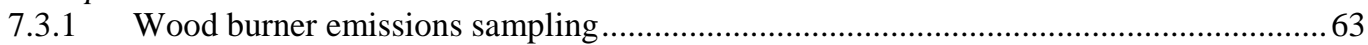

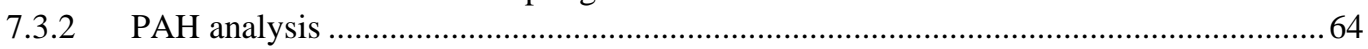

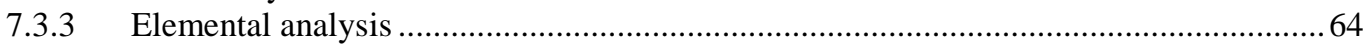

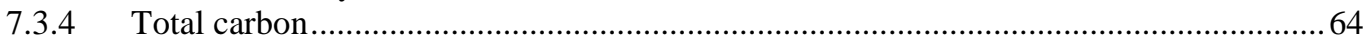

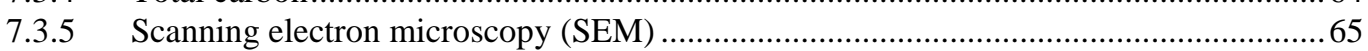

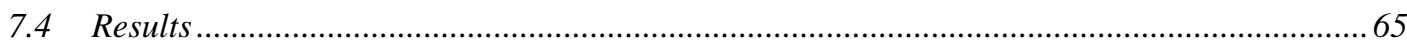

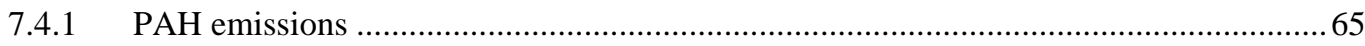

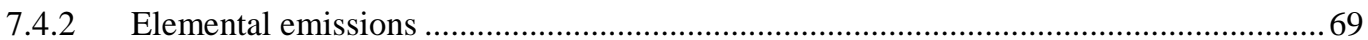

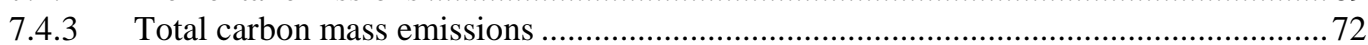

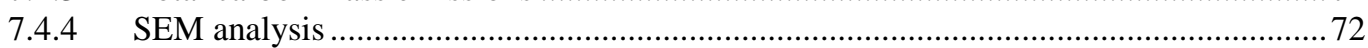




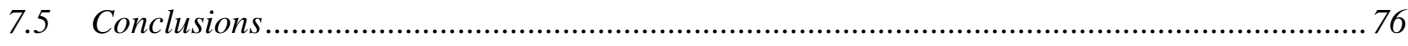

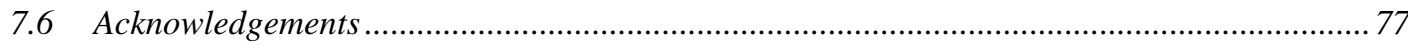

8. Carbonaceous aerosols in an urban tunnel ..........................................................78

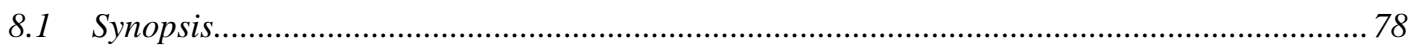

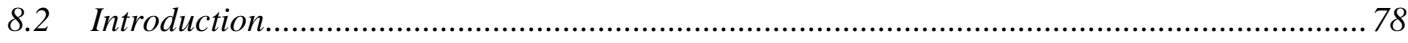

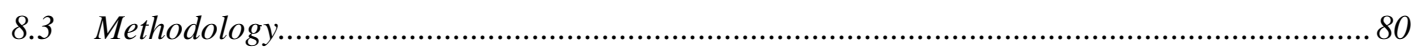

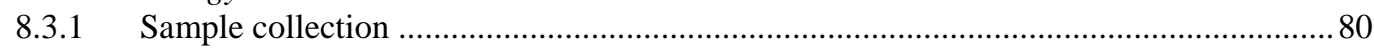

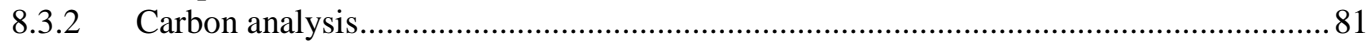

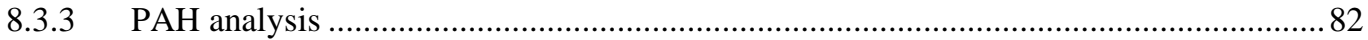

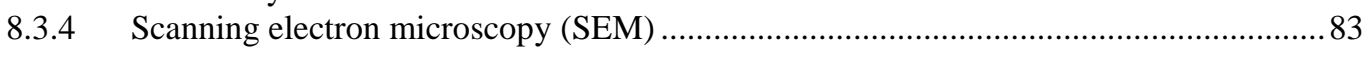

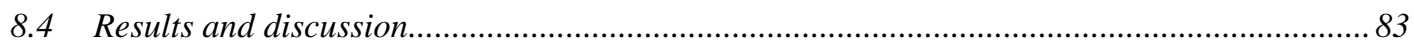

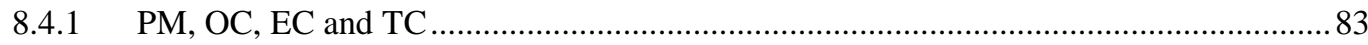

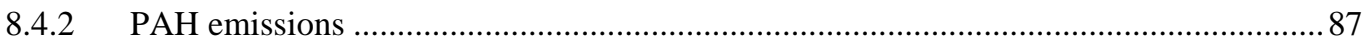

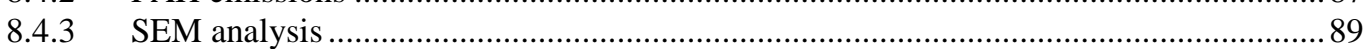

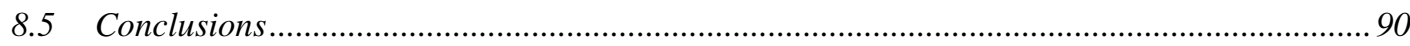

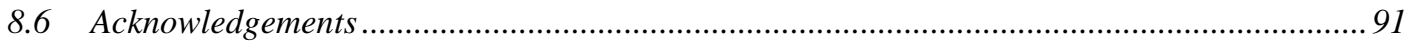

9. Masterton: Identification of particulate matter sources on an hourly timescale in a wood burning community...............................................................................92

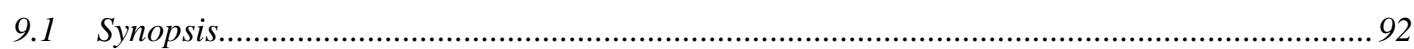

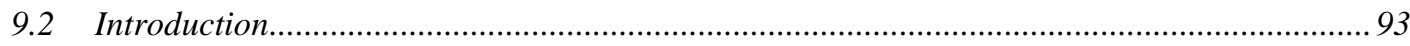

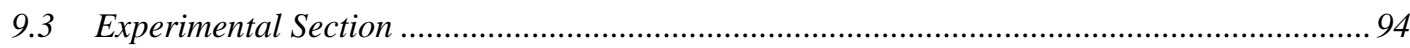

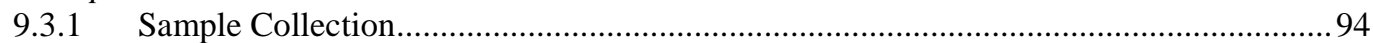

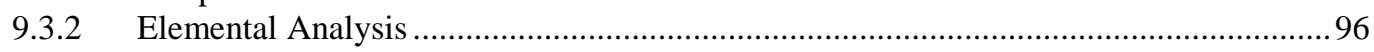

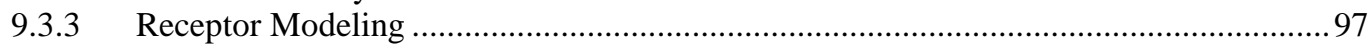

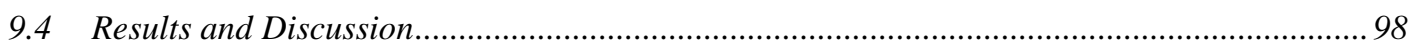

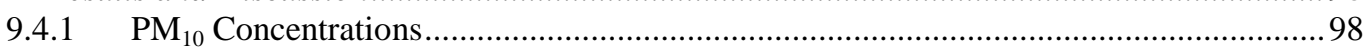

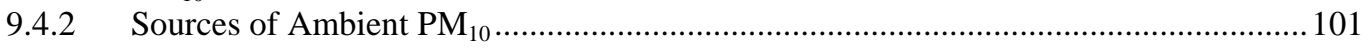

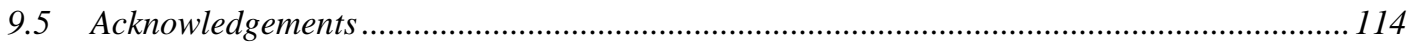

10. Nelson: Sources of particulate matter on an hourly time-scale during the winter in a small city....................................................................................................115

\begin{tabular}{|c|c|}
\hline 10.1 & 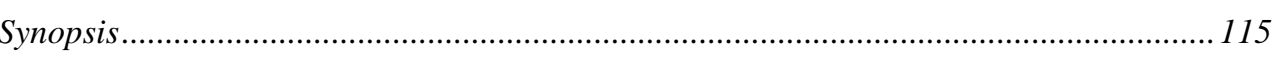 \\
\hline 10.2 & 116 \\
\hline 10.3 & Experimental Section..... \\
\hline 10.3 .1 & Sample Collection.... \\
\hline 10.3 .2 & Elemental Analysis ............. \\
\hline 10.3 .3 & Receptor Modeling ............ \\
\hline 10.4 & 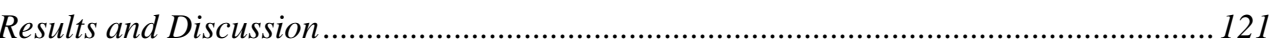 \\
\hline 10.4.1 & 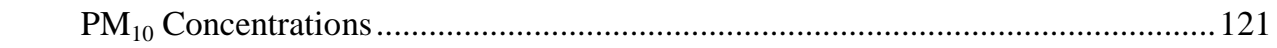 \\
\hline 10.4.2 & 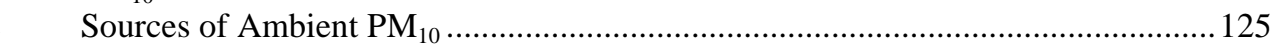 \\
\hline 10.4.3 & 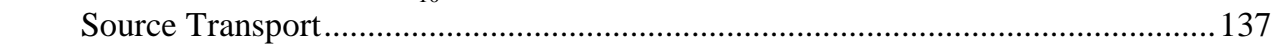 \\
\hline
\end{tabular}

11. Alexandra: Particulate matter sources on an hourly time-scale in a rural community during the winter .......................................................................................143

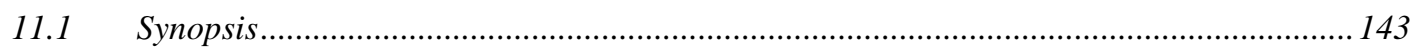

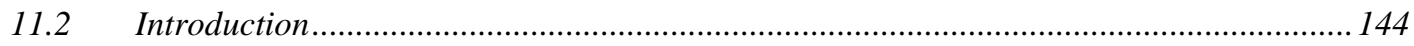




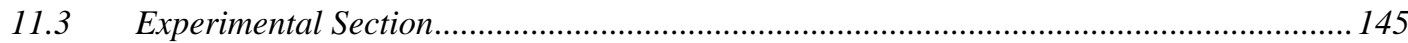

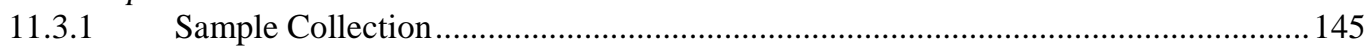

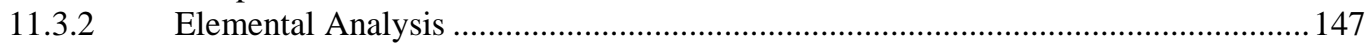

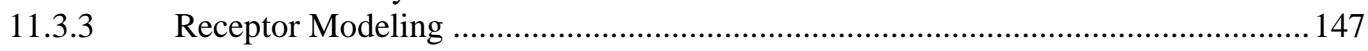

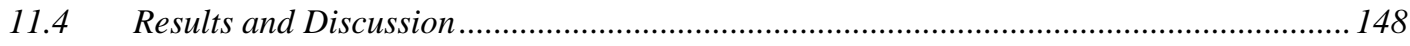

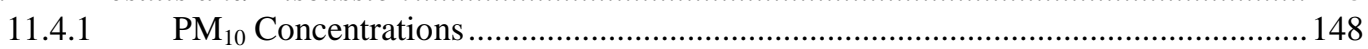

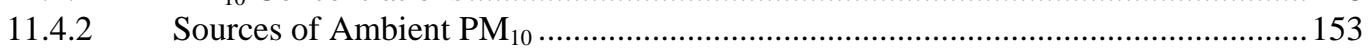

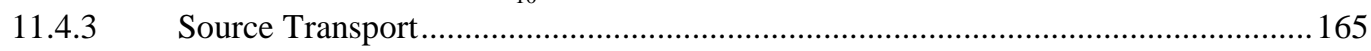

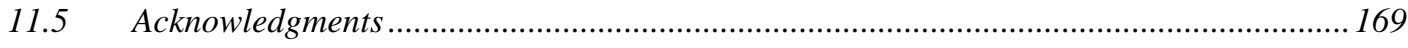

12. Concluding Remarks ...........................................................................170

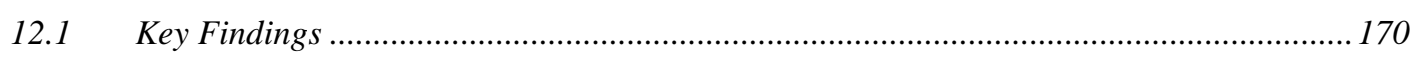

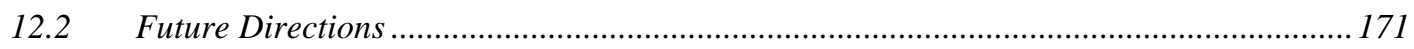

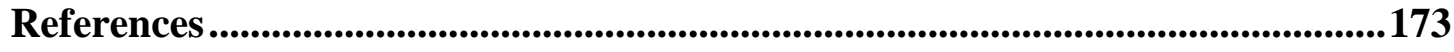

Appendix A. Analysis of Streaker filters by IBA................................................197 


\section{Table of Figures}

Figure 1.1 Typical ambient aerosol distributions by $A$ number, $B$ surface area and $\Gamma$ volume (from Colbeck and Lazaridis, 2010; originally published by Whitby, 1978) ........................................................

Figure 1.2 (a) Summary of the principle components of radiative forcing. (b) Schematic diagram of the radiative mechanisms in which aerosols affect climate change (from IPCC, 2007b)...........................8

Figure 4.1 Average hourly winter $\mathrm{PM}_{10}$ concentrations for ten urban areas across New Zealand (adapted from Trompetter et al., 2010)...........................................................................................................31

Figure 5.1 Locations of Masterton, Nelson and Alexandra. ...................................................................49

Figure 6.1 Comparison of PAH emissions during start-up between Masport Belvedere $(\mathrm{n}=20)$ and Masport LE3000 (n=8) ..........................................................................................................................5

Figure 6.2 Comparison of PAH emissions during high burn between Masport Belvedere $(n=20)$ and Masport LE3000 (n=8)

Figure 6.3 Comparison of PAH emissions during low burn between Masport Belvedere $(n=20)$ and Masport LE3000 ( $\mathrm{n}=8)$. . .59

Figure 7.1 Representative box and whisker plot of PAH emissions from pine during (a) start-up $(\mathrm{n}=4)$, (b) high burn ( $\mathrm{n}=4)$ and (c) low burn $(\mathrm{n}=4)$

Figure 7.2 Elemental mass emissions versus particulate matter emission concentrations during start-up and main burn (27 blue gum, 6 macrocarpa) . .71

Figure 7.3 SEM image of particles from the combustion of pine during start-up...............................73

Figure 7.4 SEM image of particles from the combustion of blue gum during start-up.......................74

Figure 7.5 SEM image of various particle morphologies from the combustion of blue gum during high burn $\quad 75$

Figure 7.6 SEM image of a particle cluster made up of $\mathrm{Fe}, \mathrm{Cr}$ and $\mathrm{Ni}$ .75

Figure 8.1 Plot of total carbon concentrations $\left(\mu \mathrm{g} \mathrm{m}^{-3}\right)$ determined using IMPROVE versus total carbon concentrations ( $\mu \mathrm{g} \mathrm{m}^{-3}$ ) determined using NIOSH

Figure 8.2 Plot of total carbon concentrations $\left(\mu \mathrm{g} \mathrm{m}^{-3}\right)$ determined using NIOSH and IMPROVE versus total carbon concentrations $\left(\mu \mathrm{g} \mathrm{m}^{-3}\right)$ determined using EA/IRMS . . .87

Figure 8.3 Representative SEM image of a carbonaceous particle agglomerate .90

Figure 9.1 Locations of the sampling sites within Masterton (WC-Wairarapa College; CC-Chanel College). $\quad 95$

Figure 9.2 Streaker sampler filter showing individual hourly samples (dark bands) .96 
Figure 9.3 Average hourly $\mathrm{PM}_{10}$ concentrations at the Wairarapa College and Chanel College sites from July-September 2010

Figure 9.4 Wind rose plot over the entire sampling period from the Wairarapa College site. The radial dimensions indicate the frequency (\%) of winds from each direction.

Figure 9.5 Hourly pollution roses from the Chanel College site (produced using the Openair package (Carlslaw and Ropkins, 2011)) indicating wind directions contributing the most to average hourly $\mathrm{PM}_{10}$ concentrations. The radial dimensions indicate the percentage of the total pollution that arrives from each wind sector during each one-hour period. .101

Figure 9.6 Source profiles obtained at Wairarapa College. 104

Figure 9.7 Source profiles obtained at Chanel College. 105

Figure 9.8 Plot of biomass burning contributions at Chanel College versus biomass burning contributions at Wairarapa College. 107

Figure 9.9 Hourly biomass burning source roses from the Chanel College site indicating wind directions contributing the most to average hourly $\mathrm{PM}_{10}$ concentrations. The radial dimensions indicate the percentage of the total biomass burning contribution that arrives from each wind sector during each one-hour period.

Figure 9.10 Hourly motor vehicle source roses from the Chanel College site indicating wind directions contributing the most to average hourly $\mathrm{PM}_{10}$ concentrations. The radial dimensions indicate the percentage of the total motor vehicle contribution that arrives from each wind sector during each one-hour period. 109

Figure 9.11 Hourly marine aerosol source roses from the Chanel College site indicating wind directions contributing the most to average hourly $\mathrm{PM}_{10}$ concentrations. The radial dimensions indicate the percentage of the total marine aerosol contribution that arrives from each wind sector during each one-hour period. 110

Figure 9.12 Hourly crustal matter source roses from the Chanel College site indicating wind directions contributing the most to average hourly $\mathrm{PM}_{10}$ concentrations. The radial dimensions indicate the percentage of the total crustal matter contribution that arrives from each wind sector during each one-hour period. 111

Figure 9.13 Average hourly source contributions at the Wairarapa College site.............................112

Figure 9.14 Average hourly source contributions at the Chanel College site. ....................................113

Figure 10.1 Schematic illustration of the sampling site locations in Nelson. In the Nelson case, the upwind site was located on the left, while the downwind site was located on the right. 118

Figure 10.2 Locations of the sampling sites within Nelson (NIS: Nelson Intermediate School (upwind); NCC: Nelson City Council (central); NFS: Nelson Fire Station (downwind)). The Aloft site was co-located with the NCC site. 119 
Figure 10.3 Average hourly $\mathrm{PM}_{10}$ concentrations at (a) the Nelson Intermediate School, Nelson City Council and Nelson Fire Station sites and (b) at the Nelson City Council and Aloft sites during periods when the knuckleboom was raised.

Figure 10.4 Wind rose plot over the entire sampling period from the Nelson City Council site. The radial dimensions indicate the frequency (\%) of winds from each direction.

Figure 10.5 Hourly pollution roses from the Nelson City Council site indicating wind directions contributing the most to average hourly $\mathrm{PM}_{10}$ concentrations. The radial dimensions indicate the percentage of the total pollution that arrives from each wind sector during each one-hour period...... 125

Figure 10.6 Source profiles obtained at the Nelson City Council site. ............................................130

Figure 10.7 Source profiles obtained at the Nelson Intermediate School site.................................131

Figure 10.8 Source profiles obtained at the Nelson Fire Station site. ........................................... 132

Figure 10.9 Source profiles obtained at the Aloft site................................................................133

Figure 10.10 Average hourly source contributions at the Nelson City Council site. ....................135

Figure 10.11 Average hourly source contributions at the Nelson Intermediate School site...........135

Figure 10.12 Average hourly source contributions at the Nelson Fire Station site.........................136

Figure 10.13 Average hourly source contributions at the Aloft site................................................136

Figure 10.14 Polar plots of biomass combustion contributions during the night (a) and day (b) at the NCC site. The radial dimensions indicate the wind speed in $1 \mathrm{~ms}^{-1}$ increments and the color contours indicate the average contribution to each wind direction/speed bin.

.138

Figure 10.15 Polar plots of vehicle contributions during the night (a) and day (b) at the NCC site. The radial dimensions indicate the wind speed in $1 \mathrm{~ms}^{-1}$ increments and the color contours indicate the average contribution to each wind direction/speed bin 139

Figure 10.16 Polar plots of marine aerosol contributions during the night (a) and day (b) at the NCC site. The radial dimensions indicate the wind speed in $1 \mathrm{~ms}^{-1}$ increments and the color contours indicate the average contribution to each wind direction/speed bin.

Figure 10.17 Polar plots of shipping sulfate contributions during the night (a) and day (b) at the NCC site. The radial dimensions indicate the wind speed in $1 \mathrm{~ms}^{-1}$ increments and the color contours indicate the average contribution to each wind direction/speed bin.

141

Figure 10.18 Polar plots of crustal matter contributions during the night (a) and day (b) at the NCC site. The radial dimensions indicate the wind speed in $1 \mathrm{~ms}^{-1}$ increments and the color contours indicate the average contribution to each wind direction/speed bin. 142

Figure 11.1 Schematic illustration of the sampling site locations in Alexandra. 145 
Figure 11.2 Locations of the sampling sites within Alexandra (ABC: Alexandra bowling club; GG: Girl Guides center; CODC: Central Otago District Council offices). The Aloft site was co-located with the GG site. The Clutha River flows from left to right in this figure.

Figure 11.3 Average hourly $\mathrm{PM}_{10}$ concentrations at (a) the Bowling Club, Girl Guides and Central Otago District Council office sites and (b) at the Girl Guides and Aloft sites during periods when the knuckleboom was raised. 149

Figure 11.4 Wind rose plot over the entire sampling period from the Girl Guides site. The radial dimensions indicate the frequency (\%) of winds from each direction.

Figure 11.5 Hourly pollution roses from the Girl Guides site indicating wind directions contributing the most to average hourly $\mathrm{PM}_{10}$ concentrations. The radial dimensions indicate the percentage of the total pollution that arrives from each wind sector during each one-hour period. 152

Figure 11.6 Hourly pollution roses from the Central Otago District Council site indicating wind directions contributing the most to average hourly $\mathrm{PM}_{10}$ concentrations. The radial dimensions indicate the percentage of the total pollution that arrives from each wind sector during each one-hour period.153

Figure 11.7 Source profiles obtained at the Alexandra Girl Guides site. 158

Figure 11.8 Source profiles obtained at the Alexandra Bowling Club site 159

Figure 11.9 Source profiles obtained at the Central Otago District Council offices site 160

Figure 11.10 Source profiles obtained at the Aloft site 161

Figure 11.11 Average hourly source contributions at the Alexandra Girl Guides site. 163

Figure 11.12 Average hourly source contributions at the Alexandra Bowling Club site. 164

Figure 11.13 Average hourly source contributions at the Central Otago District Council site.....164

Figure 11.14 Average hourly source contributions at the Aloft site. 165

Figure 11.15 Polar plots of biomass combustion contributions during the night (a) and day (b) at the GG site. The radial dimensions indicate the wind speed in $1 \mathrm{~ms}^{-1}$ increments and the color contours indicate the average contribution to each wind direction/speed bin. 166

Figure 11.16 Polar plots of vehicle contributions during the night (a) and day (b) at the GG site. The radial dimensions indicate the wind speed in $1 \mathrm{~ms}^{-1}$ increments and the color contours indicate the average contribution to each wind direction/speed bin

Figure 11.17 Polar plots of marine aerosol contributions during the night (a) and day (b) at the GG site. The radial dimensions indicate the wind speed in $1 \mathrm{~ms}^{-1}$ increments and the color contours indicate the average contribution to each wind direction/speed bin. 168

Figure 11.18 Polar plots of crustal matter contributions during the night (a) and day (b) at the GG site. The radial dimensions indicate the wind speed in $1 \mathrm{~ms}^{-1}$ increments and the color contours indicate the average contribution to each wind direction/speed bin. 168 
Figure A.1. Schematic illustration of the air particulate chamber detector layout (PIXE: particleinduced x-ray emission; PESA: particle elastic scattering; HPGE(PIGE): particle-induced gamma ray emission; RBS: Rutherford backscattering) (a) and a schematic illustration of the beam line (b).......198 


\section{Table of Tables}

Table 2.1 Measurement methods used to determine aerosol composition..........................................12

Table 6.1 Particulate emission factors for Masport Belvedere ( $n=60)$ and Masport LE3000 (n=24).

Table 6.2 PAH emission factors for Masport Belvedere $(n=60)$ and Masport LE3000 (n=24)......55

Table 7.1 PAH emission factors for softwood and hardwood ....................................................66

Table 7.2 BaP emission factors for all wood types studied under each operating condition ...........67

Table 7.3 Emission factors of elements (mg/kg wood) above their respective detection limits .....70

Table 8.1 Average OC, EC and TC concentrations (and associated standard deviations in parentheses) obtained from NIOSH and IMPROVE thermal/optical protocols along with OC/EC ratios and percentage of each component relative to fine particle concentration

Table 8.2 Average particle-phase PAH concentrations (and associated standard deviations in parentheses) obtained from TD/GC/MS 88

Table 9.1 Parameters used for PMF analyses and diagnostics obtained from the analyses for Wairarapa College and Chanel College.

Table 9.2 $\quad \mathrm{PM}_{10}\left(\mu \mathrm{g} \mathrm{m}^{-3}\right)$, BC $\left(\mathrm{ng} \mathrm{m}^{-3}\right)$ and elemental $\left(\mathrm{ng} \mathrm{m}^{-3}\right)$ data from the Wairarapa College site

Table 9.3 $\quad \mathrm{PM}_{10}\left(\mu \mathrm{g} \mathrm{m}^{-3}\right)$, BC $\left(\mathrm{ng} \mathrm{m}^{-3}\right)$ and elemental $\left(\mathrm{ng} \mathrm{m}^{-3}\right)$ data from the Chanel College site....

Table 10.1 Parameters used for PMF analyses and diagnostics obtained from the analyses for each of the sites

Table 10.2 $\mathrm{PM}_{10}\left(\mu \mathrm{g} \mathrm{m}^{-3}\right)$, BC $\left(\mathrm{ng} \mathrm{m}^{-3}\right)$ and elemental $\left(\mathrm{ng} \mathrm{m}^{-3}\right)$ data from the Nelson Intermediate School site (1081 samples) 126

Table 10.3 $\quad \mathrm{PM}_{10}\left(\mu \mathrm{g} \mathrm{m}^{-3}\right), \mathrm{BC}\left(\mathrm{ng} \mathrm{m}^{-3}\right)$ and elemental $\left(\mathrm{ng} \mathrm{m}^{-3}\right)$ data from the Nelson City Council site (1034 samples) 127

Table 10.4 $\mathrm{PM}_{10}\left(\mu \mathrm{g} \mathrm{m}^{-3}\right)$, BC $\left(\mathrm{ng} \mathrm{m}^{-3}\right)$ and elemental $\left(\mathrm{ng} \mathrm{m}^{-3}\right)$ data from the Nelson Fire Station site (816 samples).

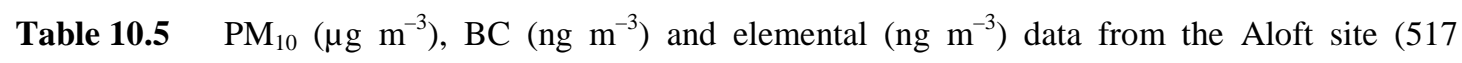
samples)

Table 11.1 Parameters used for PMF analyses and diagnostics obtained from the analyses for each of the sites

Table 11.2 $\mathrm{PM}_{10}\left(\mu \mathrm{g} \mathrm{m}^{-3}\right)$, BC $\left(\mathrm{ng} \mathrm{m}^{-3}\right)$ and elemental $\left(\mathrm{ng} \mathrm{m}^{-3}\right)$ data from the Alexandra Bowling Club site (892 samples) 
Table 11.3 $\quad \mathrm{PM}_{10}\left(\mu \mathrm{g} \mathrm{m}^{-3}\right)$, BC $\left(\mathrm{ng} \mathrm{m}^{-3}\right)$ and elemental ( $\mathrm{ng} \mathrm{m}^{-3}$ ) data from the Girl Guides site (1027 samples)

Table 11.4 $\mathrm{PM}_{10}\left(\mathrm{gg} \mathrm{m}^{-3}\right)$, BC $\left(\mathrm{ng} \mathrm{m}^{-3}\right)$ and elemental ( $\mathrm{ng} \mathrm{m}^{-3}$ ) data from the Central Otago District Council site (940 samples) 156

Table 11.5 $\mathrm{PM}_{10}\left(\mu \mathrm{g} \mathrm{m}^{-3}\right)$, BC $\left(\mathrm{ng} \mathrm{m}^{-3}\right)$ and elemental $\left(\mathrm{ng} \mathrm{m}^{-3}\right)$ data from the Aloft site (845 samples)

Table A.1. Sample program worksheet for the automated analysis of Streaker filters..... 199 


\section{List of Abbreviations}

AAS Atomic absorption spectroscopy

AAQG Ambient air quality guideline

AC Automated colorimetry

AMS Aerosol mass spectrometry

BAM Beta-particle attenuation monitor

BaP Benzo[a]pyrene

BC Black carbon

CCN Cloud condensation nuclei

CMB Chemical mass balance

EA/IRMS Elemental analysis-isotope ratio mass spectrometry

EC Elemental carbon

GC Gas chromatography

GC/MS Gas chromatography-mass spectrometry

HAPiNZ Health and Air Pollution in New Zealand

HPLC High-performance liquid chromatography

IBA Ion beam analysis

IC Ion chromatography

ICP-AES Inductively coupled plasma-atomic emission spectroscopy

ICP-MS Inductively coupled plasma-mass spectrometry

IMPROVE Interagency monitoring of protected visual environments

INAA Instrumental neutron activation analysis

IPCC Intergovernmental Panel on Climate Change

LC/MS Liquid chromatography-mass spectrometry

LOD Limit of detection

NES National Environmental Standard

NIOSH National Institute for Occupational Safety and Health

NO $_{\mathbf{x}} \quad$ Nitrogen oxides

OC Organic carbon

PAH Polycyclic aromatic hydrocarbon

PCA Principal components analysis 


\begin{tabular}{|c|c|}
\hline PESA & Particle elastic scattering analysis \\
\hline PIGE & Particle-induced gamma-ray emission \\
\hline PIXE & Particle-induced X-ray emission \\
\hline PM & Particulate matter \\
\hline $\mathbf{P M}_{10}$ & Particulate matter less than $10 \mu \mathrm{m}$ in aerodynamic diameter \\
\hline $\mathbf{P M}_{10-2.5}$ & Particulate matter with aerodynamic diameters $2.5 \mu \mathrm{m}<d<10 \mu \mathrm{m}$ \\
\hline $\mathbf{P M}_{2.5}$ & Particulate matter less than $2.5 \mu \mathrm{m}$ in aerodynamic diameter \\
\hline PMF & Positive matrix factorization \\
\hline QA/QC & Quality assurance-quality control \\
\hline RBA & Rutherford backscattering analysis \\
\hline $\mathbf{R C M}$ & Reconstructed mass \\
\hline RMA & Resource Management Act \\
\hline SEM-EDS & Scanning electron microscopy-energy dispersive spectroscopy \\
\hline $\mathbf{S} / \mathbf{N}$ & Signal-to-noise \\
\hline SFU & Stacked filter unit \\
\hline $\mathbf{S O}_{\mathrm{x}}$ & Sulfur oxides \\
\hline SVOC & Semi-volatile organic compound \\
\hline TC & Total carbon \\
\hline TD/GC/MS & Thermal desorption-gas chromatography-mass spectrometry \\
\hline TEOM & Tapered element oscillating microbalance \\
\hline TMO & Total manganese oxidation \\
\hline TOR & Thermal optical reflectance \\
\hline TOT & Thermal optical transmission \\
\hline USA & United States of America \\
\hline USEPA & United States Environmental Protection Agency \\
\hline VOC & Volatile organic compound \\
\hline XRF & $\mathrm{X}$-ray fluorescence \\
\hline
\end{tabular}




\section{Atmospheric Aerosols - An Introduction}

\subsection{Background}

The impacts of atmospheric aerosols on public health, climate and atmospheric composition are currently among the most widely studied topics in environmental science research. In particular, the issue of climate change has gained the widespread attention of the general public. The central importance of climate change to the future of all Earth's inhabitants was recognized in 2007, when the Nobel peace prize was jointly awarded to Al Gore for his documentary on climate change and the scientists on the Intergovernmental Panel on Climate Change (IPCC), who completed a comprehensive overview of the current scientific knowledge in regards to climate change (IPCC 2007a). With the overwhelming majority of the scientific community accepting that global warming is a well-documented phenomenon; many uncertainties still exist with regards to the role of anthropogenic activities and the rate of warming. Currently, the role of atmospheric aerosols on global warming presents the largest uncertainties in the quantification and modeling of global climate change.

\subsection{General Characteristics of Atmospheric Aerosols}

The origins of the term aerosol can be traced back to research performed during World War I to denote a system of particles of ultra-microscopic size dispersed in a gas, as suggested by Professor F. G. Donnon (Whytlaw-Gray 1923), although A. Schmauss was the first to publish the term (Colbeck and Lazaridis 2010). A more detailed discussion of aerosol was published by Gibbs (1924). An aerosol is generally defined as a stable suspension of solid and liquid particles in a gas (Hinds 1999). Examples of atmospheric aerosols include dust, smog, smoke and fog. The most evident examples of aerosols in the atmosphere are clouds, but traditionally the term aerosol refers to suspended particles that contain a large proportion of condensed matter other than water, so clouds are considered as a separate phenomenon (Pöschl 2005). 


\subsubsection{Size Distribution of Atmospheric Aerosol Particles}

Aerosol particle sizes span five orders of magnitude, ranging from aerodynamic diameters of several nanometers to $100 \mu \mathrm{m}$. Particles that are much greater than $100 \mu \mathrm{m}$ do not stay airborne long enough to be measured and observed as aerosols. Despite the large size range of atmospheric particles, they are generally grouped into one of two size modes: a coarse mode, made up of particles with an aerodynamic diameter $2.5 \mu \mathrm{m}<d<10 \mu \mathrm{m}$ (generally denoted as $\mathrm{PM}_{10-2.5}$ ), or a fine mode, made up of particles with aerodynamic diameters $<2.5 \mu \mathrm{m}$ (generally denoted as $\mathrm{PM}_{2.5}$ ). This differentiation is important because particles within each of these modes typically originate from different sources and have different physicochemical properties. Fine and coarse mode particles also undergo different atmospheric transformations and are removed from the atmosphere via different mechanisms. Generally fine mode particles are formed by combustion processes or gas-to-particle conversion, while coarse mode particles are produced through mechanical abrasion processes like entrainment of road dust, windblown soils and sea spray.

Fine mode particles can be further divided into several subcategories (Colbeck and Lazaridis 2010):

- Nucleation mode: those particles with diameter $<10 \mathrm{~nm}$ which are formed through nucleation processes.

- Aitken mode: particles with diameter $10 \mathrm{~nm}<d<100 \mathrm{~nm}$ which originate from vapor nucleation or growth of pre-existing particles due to condensation.

- Accumulation mode: particles with diameter $0.1 \mu \mathrm{m}<d<1 \mu \mathrm{m}$ which are formed from the coagulation of smaller particles or the condensation of vapor constituents.

- Ultrafine particles: particles in the Aitken and nucleation modes.

As discussed above, particles in the atmosphere have a distribution of sizes and for their description, Whitby et al. (1972a; 1972b) first concluded that typical particle size distributions could be explained by two log-normal curves superimposed on each other with a minimum in the 1-3 $\mu \mathrm{m}$ range. Figure 1.1 presents typical atmospheric aerosol distributions by number, surface area and volume. The form of aerosol size distributions can be very different, even though the distributions contain the same particle modes, depending on how the distribution is plotted. For example, plotting a particle number distribution places more emphasis on smaller particles, 
which are far more abundant, while a particle mass distribution places more emphasis on larger, heavier particles that are less abundant.

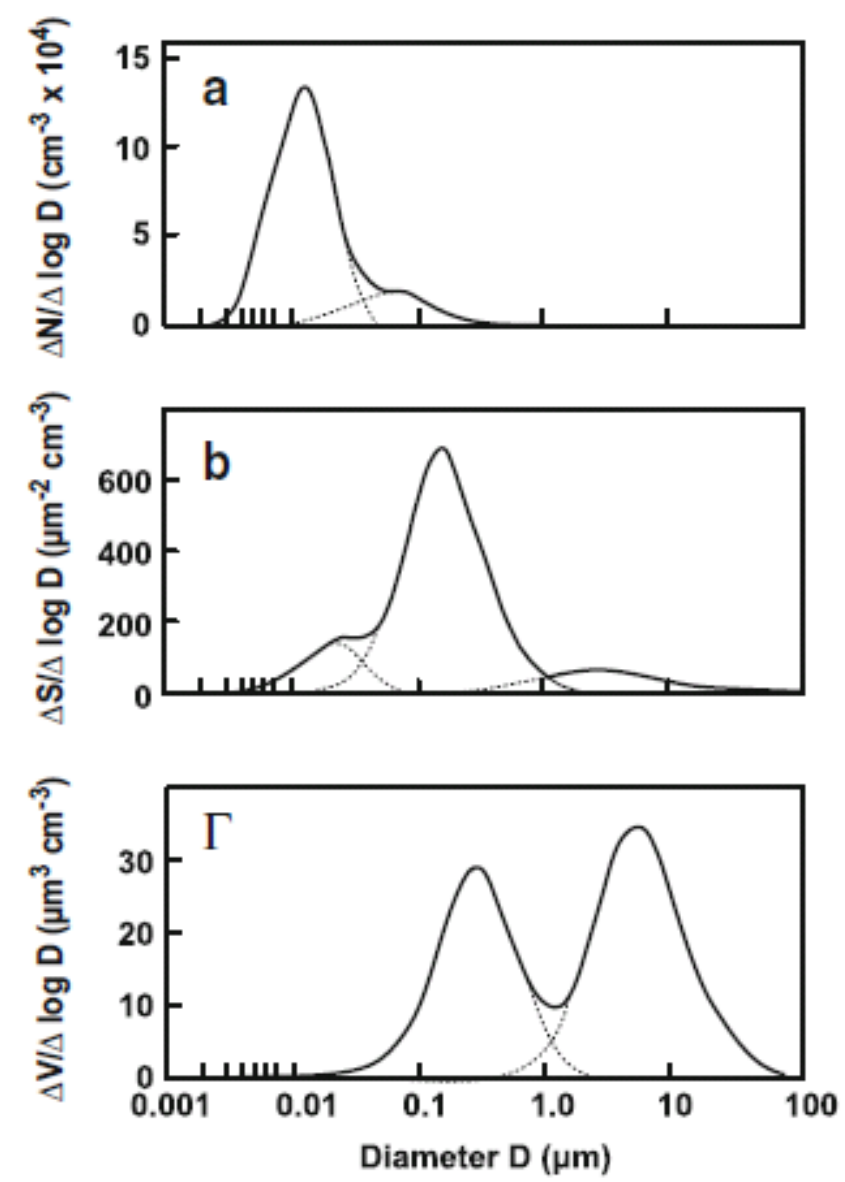

Figure 1.1 Typical ambient aerosol distributions by $A$ number, $B$ surface area and $\Gamma$ volume (from Colbeck and Lazaridis, 2010; originally published by Whitby, 1978)

From Figure 1.1 it is clear that ultrafine particles dominate the aerosol number distribution. The volume distribution is bimodal and displays a greater emphasis on larger particles. If the particle density is known a mass distribution can be derived from the volume distribution.

Particles in the fine mode tend to have long residence times in the atmosphere and can be transported large distances in the atmosphere. Coarse mode particles tend to have short residence times on the order of days in the atmosphere, settling out of the atmosphere due to gravity. Particle lifetimes and their removal from the atmosphere are discussed in more detail in Chapter 1.2.3. The large differences in residence times between coarse and fine mode particles means that fine particles collected at a monitoring site may have been transported from a distant source, while 
coarse mode particles are more likely to originate from local sources, creating a useful distinction for interpreting the analytical results of size-resolved aerosol particles.

\subsubsection{Sources and Composition of Atmospheric Aerosol Particles}

Aerosol particles in the atmosphere originate from both natural and anthropogenic sources. Major natural aerosol sources include volcanic activity, sea spray and mineral dust. Anthropogenic sources of atmospheric particles include industrial emissions, biomass burning, combustion of fossil fuels and non-industrial fugitive sources, for example, construction work. Sometimes, distinguishing between natural and anthropogenic sources is not clear. For example, smoke arising from natural wildfires has been categorized as anthropogenic in origin and mineral dust originating from agriculturally eroded regions has been classified as a natural source (Colbeck and Lazaridis 2010).

Particles in the atmosphere are classified as either primary or secondary based on their formation mechanism. Primary particles are emitted directly into the atmosphere, while secondary particles are formed from gas phase precursors. Secondary particle formation is initiated by photo-oxidation reactions of organic and inorganic gas phase species (Jimenez et al. 2009). These reactions tend to lower the vapor pressure of the product, resulting in condensation onto existing particles or undergoing nucleation to form new particles.

Fine particles are typically made up of inorganic ions such as sulfates, nitrates and ammonium, as well as combustion-derived products like organic carbon (OC; eg. hydrocarbons), elemental carbon (EC) and trace metals (Malm et al. 1994). Sulfate, nitrate and ammonium particles are all secondary particles formed from reactions of $\mathrm{SO}_{\mathrm{x}}, \mathrm{NO}_{\mathrm{x}}$ and $\mathrm{NH}_{3}$ in the atmosphere. Sources of these precursor gases include industrial emissions, such as coal-fired power plants and oil refineries, motor vehicle emissions, volcanic emissions, lightning discharges and biogenic sources. Animal husbandry and fertilization are also large $\mathrm{NH}_{3}$ emitters.

Coarse particles are formed from mechanical abrasion and wind action giving rise to particles that normally consist of minerals of crustal matter, elements present in sea water and organic matter from abraded plant detritus and pollens (Jacobson et al. 2000). 


\subsubsection{Particle Lifetimes in the Atmosphere}

The lifetime of a particle in the atmosphere is determined by the efficiency of the removal mechanism acting on that particle. Removal of particles from the atmosphere occurs by either wet or dry deposition. Wet deposition of particles occurs by precipitation scavenging aerosol particles from the atmosphere or aerosol particles acting as cloud condensation nuclei. Wet deposition is the main sink of atmospheric aerosol particles. Deposition of particles without precipitation is termed dry deposition and dry deposition occurs by convective transport, diffusion and finally adhesion to the Earth's surface. While this process is far less important on a global scale as a sink for aerosol particles, it is highly relevant with respect to local air quality, health effects and the soiling of buildings and cultural monuments.

The lifetimes of aerosol particles are also a function of their size, chemical composition, density and surrounding meteorological conditions. In general, particles have characteristic residence times that range from hours to weeks (Raes et al. 2000; Williams et al. 2002).

\subsection{Health Effects of Atmospheric Aerosols}

The negative impacts of atmospheric aerosols on human health were widely recognized by the early 1900s and Bachmann (2007) provides an exceptionally detailed account of these very early days of our understanding of the health impacts of aerosols. The health impacts of aerosols were ultimately highlighted by two major incidents in the mid-1900s. The first of these incidents occurred from October 30-31, 1948 in Donora, PA, USA. This pollution episode killed 20 people and made thousands of people ill (Seinfeld 2004). Following this episode, the "London Killer Fog” of December 1952 firmly established the negative health impact of air pollution. During this episode, an inversion caused large amounts of pollution from coal burning to be trapped in the city and resulted in approximately 4000 fatalities (Stone 2002). These events, along with more local observations and measurements, led many countries to implement legislation in an effort to reduce air pollution. In New Zealand, the National Environmental Standards (NES) for air quality were introduced in October 2004. The 14 standards include: seven standards banning activities that discharge significant quantities of dioxins and other toxins into the air; five standards for ambient air quality; a design standard for new wood burners installed in urban 
areas; and a requirement for all landfills with over 1 million tonnes of refuse to collect greenhouse gas emissions. The NES prescribes a mean daily maximum concentration of $50 \mu \mathrm{g} \mathrm{m}^{-3}$ for $\mathrm{PM}_{10}$. More details about the NES are provided in Chapter 4.3.

In an effort to better understand the health impacts of particulate matter (PM) on human health, studies in various cities have confirmed a positive relationship between PM and both hospital admissions for respiratory illness and mortality rates (Pope 1989; Pope 1996; Schwartz 1996; Klemm et al. 2000; Tsai et al. 2003). Possibly the most well-known study confirming the relationship between PM concentrations and health impacts was the Harvard Six Cities Study (Dockery et al. 1993). In their study, researchers at Harvard studied a cohort of people in six different cities over a period of 14 - 16 years and found a strong correlation between mortality rates and the concentrations of PM that individuals were exposed to. Since these early studies, additional approaches with more advanced methodologies have begun to link adverse health effects to specific sources (Grahame and Schlesinger 2007). As an example, a study in Southern California observed an increased risk of children developing asthma if they resided within $75 \mathrm{~m}$ of a major roadway during the first two years of their lives (McConnell et al. 2006). Chemical analysis of PM can be used to determine which chemical species, like polycyclic aromatic hydrocarbons (PAHs) and trace metals may be present and how they could interact with the body (Lewtas 1993; Hunt et al. 2003). Particle size has also been determined to play an important role on adverse health effects. Ultrafine particles in particular have been found to pose the most severe effects. These particles are able to penetrate very deeply into the body and can even be absorbed directly into the bloodstream (Gwinn and Vallyathan 2006). Ultrafine particles have the highest number concentration and a large surface area, meaning they have the capacity to carry many toxic species deep into the body where they can target organs like the liver or the brain (Hinds 1999; Nel 2005). More discussion regarding the relationship between particulate matter and health effects can be found in Vedal (1997), Pope and Dockery (2006) and Mauderly and Chow (2008).

\subsection{Environmental and Climate Effects of Atmospheric Aerosols}

In addition to the adverse health effects of PM, one of the most obvious impacts of PM pollution is the formation of "haze" or a reduction in visibility. Degradation of visibility is well known to occur in urban environments at the source 
of anthropogenic pollution, particularly in large cities like Los Angeles, but it can also occur in the pristine environments of national parks (Appel et al. 1985; Malm et al. 1994; Malm and Day 2000). Reduction of atmospheric visibility is largely caused by the scattering of light by particles with diameters that are close in size to the wavelength of visible light (particles in the accumulation mode) (Seinfeld and Pandis 2006). Due to the aesthetic value of a clear view, some countries, like the USA, have legislation which includes visibility as an air quality related value. More discussion on visibility and the US Regional Haze Rule can be found in Watson (2002a). In New Zealand, visibility is regarded as an important amenity; therefore a reduction in visibility due to air pollution must be considered and managed accordingly under the Resource Management Act 1991.

Less obvious impacts of atmospheric particles include the effects of particles on man-made structures and environmental media. In these instances, particles are deposited by wet or dry deposition, and the effects of the deposited particles are directly related to their chemical properties. Acidic aerosols, for example, can corrode metal structures and degrade building materials like concrete, limestone and marble, reducing structural integrity (Seinfeld and Pandis 2006). Aerosols that contain toxic metals or organic compounds can be deposited on land and in water bodies, where they directly affect flora and fauna, and indirectly, through bio-accumulation and ingestion, affect human health. Soot particles formed through combustion processes can spoil surfaces and increase cleaning and maintenance costs.

Aerosol particles in the atmosphere also provide surfaces for surface-mediated heterogeneous reactions with gases. The reactions can have a significant impact on atmospheric composition. For example, the Antarctic ozone hole is driven by chemical reactions associated with small particulates (Solomon 1999). Similarly, heterogeneous oxidation of particle-phase PAHs to yield oxygenated PAHs causes a significant increase in the toxicity of the resulting particles (George and Abbatt 2010).

Interest in atmospheric aerosols also extends to their influence on global climate change. The measure by which a climate factor alters the balance of Earth's atmosphere is given by the radiative forcing (IPCC 2007b). A positive radiative forcing represents a warming of the climate; conversely, a negative radiative forcing represents a cooling. Figure 1.2 presents the radiative forcing of aerosols and greenhouse gases and illustrates how aerosols can influence climate. The large uncertainty bars on the negative radiative forcing of aerosols in Figure 1.2(a) indicate 
that the absolute effects of aerosols have a high uncertainty, as reported by the IPCC. The warming effects of greenhouse gases like $\mathrm{CO}_{2}, \mathrm{CH}_{4}, \mathrm{~N}_{2} \mathrm{O}$ and halocarbons, on the other hand, are well-characterized and more precisely known.
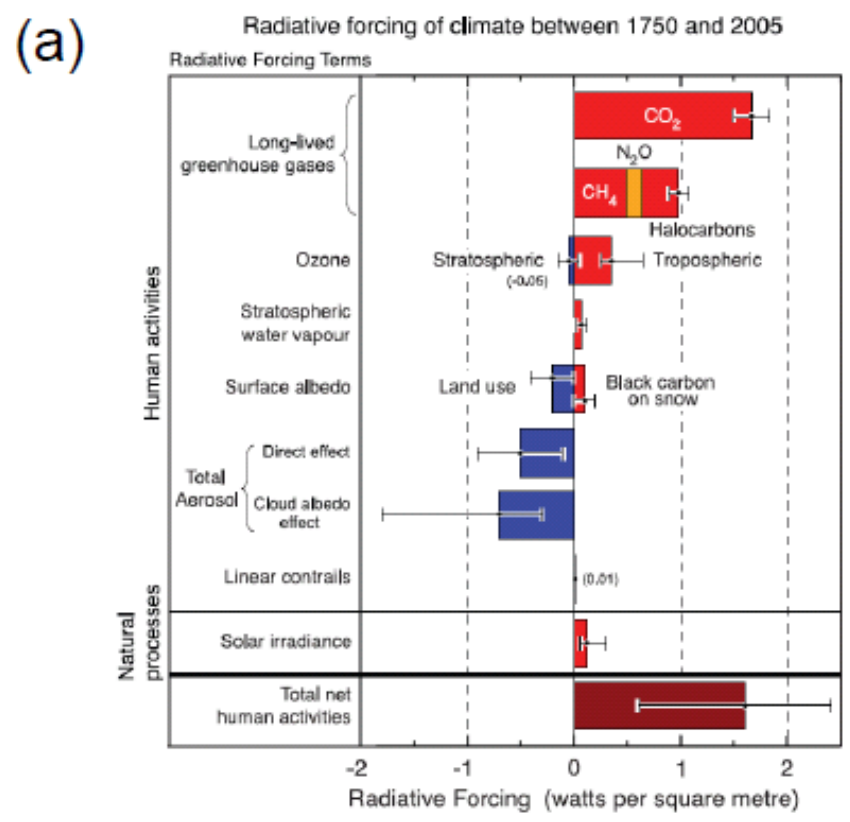

(b)

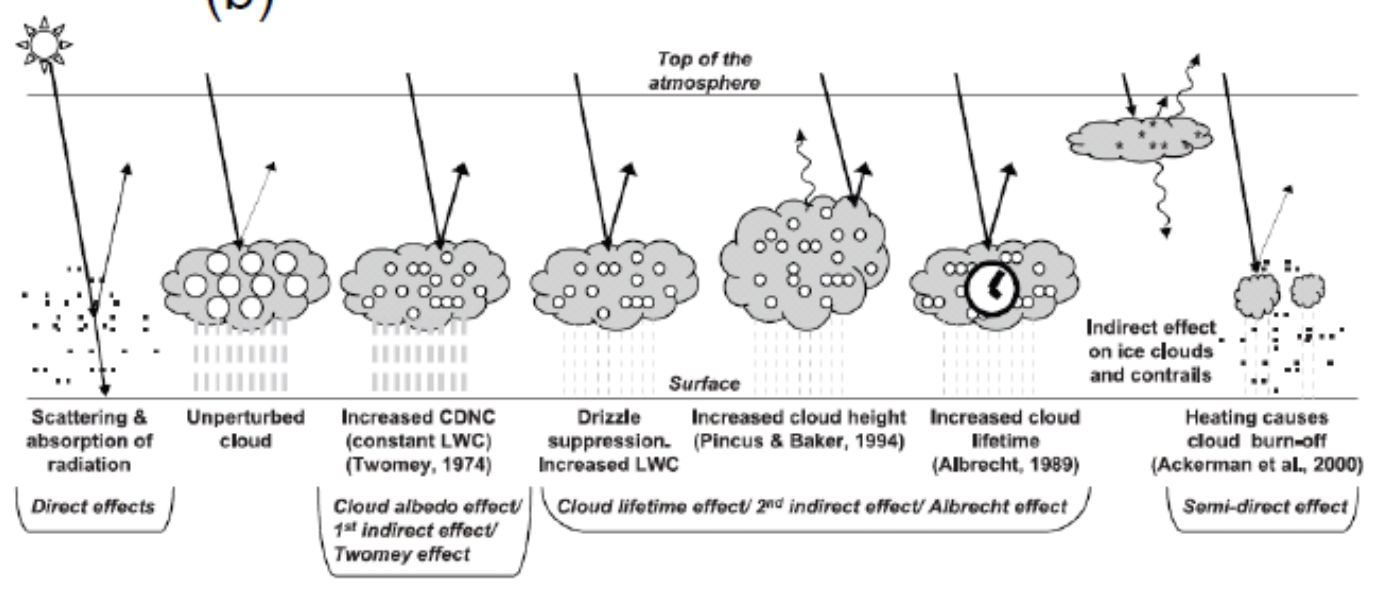

Figure 1.2 (a) Summary of the principle components of radiative forcing. (b) Schematic diagram of the radiative mechanisms in which aerosols affect climate change (from IPCC, 2007b)

The complex influence of aerosols on climate is illustrated in Figure 1.2 (b). Aerosols can have direct, indirect and semi-direct influences on climate. The direct scattering or absorption of solar radiation by aerosols is likely the simplest mechanism by which aerosols can influence climate. The scattering or reflection of incoming solar radiation causes atmospheric cooling, while absorption of radiation by aerosols causes a warming of the atmosphere. As an indirect effect, aerosol particles can act as cloud condensation nuclei (CCN) for cloud droplets, affecting cloud formation and 
lifetimes (Haywood and Boucher 2000). High particle concentrations lead to increased cloud droplet concentrations and brightness (albedo) of the cloud. A brighter cloud will reflect more radiation back to space, causing a cooling of the atmosphere (Twomey 1974; Twomey 1977). High cloud droplet concentrations also means there is less water per droplet, decreasing cloud precipitation efficiency and increasing cloud lifetime and thickness (Albrecht 1989). This effect also increases the reflection of radiation, and causes a cooling of the atmosphere. Alternatively, absorbing aerosols can heat and evaporate clouds, reducing cloud coverage and increasing the amount of radiation that reaches the Earth's surface (semi-direct effect) (Ackerman et al. 2000). The influence of atmospheric aerosols on global climate is a complex and diverse problem that will require more research before the large uncertainties in Figure 1.2 (a) can be reduced. 


\section{Atmospheric Aerosols - Analysis}

The collection and analysis of atmospheric aerosols is of central importance in atmospheric studies. Not only does particle analysis provide compositional information relevant in assessing possible health impacts of PM, but it also provides data that is absolutely essential for source apportionment analyses to determine the various sources contributing to ambient concentrations of aerosol. Aerosol collection and analysis is also important for regulatory authorities to determine their level of compliance with national air quality standards. Due to their central importance, this chapter will provide a review of commonly used techniques for particle collection and the compositional analysis of PM samples collected on filters.

\subsection{Collection of Atmospheric Aerosols}

Atmospheric aerosols are typically collected using filter-based methods following particle size differentiation. Filters composed of Teflon, Nylon, polycarbonate and quartz are commonly used for the collection of PM and the choice of filter composition is important when further characterization of the collected PM is necessary.

Particle sampling systems are generally simplistic in concept; their main requirement is maintaining a flow of ambient air through the system that is sufficient to achieve the desired particle size cut-points. Sampling systems are also required to measure the volume of air sampled over the relevant period. Some continuous particle sampling systems are available that are typically used for ambient air quality compliance monitoring and not for subsequent particle analysis. Examples of these types of systems are the Tapered Element Oscillating Microbalance (TEOM), which determines particle mass based on changes in the oscillating frequency of a small quartz crystal microbalance, and the beta-particle attenuation monitor (BAM), that determines collected particle mass by the attenuation of beta particle transmission through filter tape as it collects PM.

Because reliability and reproducibility in particle measurements is critical, quality assurance and quality control (QA/QC) are integral components in particle measurements (Hopke 1999b). There are a number of prescriptive monitoring methods, including QA/QC procedures for $\mathrm{PM}_{10}$ and $\mathrm{PM}_{2.5}$ monitoring that have been 
introduced, mainly through the United States Environmental Protection Agency (USEPA). Standard methods in Australia and New Zealand are essentially equivalent to those adopted by the USEPA, with minor modifications appropriate to the local conditions (Davy 2007). Prior to collection, filters are required to be conditioned in a temperature and humidity controlled environment for 24 hours before being weighed and conditioned again for re-weighing after exposure of the filter. Filter conditioning is necessary because moisture absorption and desorption can significantly affect gravimetric results, becoming a source of uncertainty when calculating the ambient concentration of particles.

\subsection{Analysis of Atmospheric Aerosols}

Table 2.1 provides an overview of the methods commonly used to characterize PM samples collected on filters and the chemical species that can be characterized in the aerosol (Landsberger and Creatchman 1999; Wilson et al. 2002; Davy 2007). Initially, filters are typically subjected to gravimetric analysis to determine the PM concentration as a mass per unit volume (generally reported as $\mu \mathrm{g} \mathrm{m}^{-3}$ ). Some compounds, for example volatile organic compounds (VOCs) or semi-volatile organic compounds (SVOCs), may not be analyzed gravimetrically due to difficulties associated with their high volatilities. To determine mass concentrations of these species, filters can be directly desorbed or aspirated for analysis by gas chromatography (GC) or high performance liquid chromatography (HPLC) using standards for quantification. The sections below provide a brief discussion of each of the analytical methods presented in Table 2.1. 


\begin{tabular}{|c|c|}
\hline Chemical species & Analysis method \\
\hline Particle mass & Gravimetric \\
\hline Elements (Na to U) & $\begin{array}{l}\text { Atomic Absorption Spectroscopy (AAS) } \\
\text { Inductively Coupled Plasma - Atomic } \\
\text { Emission Spectroscopy (ICP-AES) } \\
\text { Inductively Coupled Plasma - Mass } \\
\text { Spectrometry (ICP-MS) } \\
\text { X - ray Fluorescence (XRF) } \\
\text { Ion Beam Analysis (IBA) } \\
\text { Instrumental Neutron Activation Analysis } \\
\text { (INAA) }\end{array}$ \\
\hline $\begin{array}{l}\text { Water soluble cations: } \mathrm{NH}_{4}^{+}, \mathrm{Na}^{+}, \mathrm{K}^{+} \text {, } \\
\mathrm{Mg}^{2+}, \mathrm{Ca}^{2+} \\
\text { Water soluble anions: } \mathrm{F}^{-}, \mathrm{Br}^{-}, \mathrm{Cl}^{-}, \mathrm{NO}_{3}^{-} \text {, } \\
\mathrm{SO}_{4}^{2-}\end{array}$ & $\begin{array}{l}\text { Ion Chromatography (IC) } \\
\text { Automated Colorimetry (AC) } \\
\text { AAS - cations only }\end{array}$ \\
\hline Carbon: OC, EC, BC & $\begin{array}{l}\text { Thermal/Optical Reflectance (TOR) } \\
\text { Thermal/Optical Transmission (TOT) } \\
\text { Thermal Manganese Oxidation (TMO) } \\
\text { Light Transmission/Reflection } \\
\text { Isotope Analysis } \\
\text { Gas Chromatography - Mass } \\
\text { Spectrometry (GC/MS) - organics only } \\
\text { Liquid Chromatography (LC) - organics } \\
\text { only }\end{array}$ \\
\hline $\begin{array}{l}\text { Single-particle size, morphology and } \\
\text { composition }\end{array}$ & $\begin{array}{l}\text { Scanning Electron Microscopy - Energy } \\
\text { Dispersive Spectroscopy (SEM) }\end{array}$ \\
\hline
\end{tabular}

\subsubsection{Atomic Absorption Spectroscopy (AAS)}

AAS is performed by extracting the PM off a filter and into a solvent, followed by injection of the extract into an oxy-acetylene flame where the elements are vaporized. Since elements absorb light at different wavelengths in the visible spectrum, a light beam with wavelengths specific to the elements being measured is directed through the flame to be detected by a monochromator. By comparing the light absorbed by the flame containing the extract with the absorption from known standards, the elemental concentrations can be quantified. AAS has low detection limits and is a useful complement to other methods that have difficulty quantifying low molecular weight species like Be, $\mathrm{Na}$ and Mg (Wilson et al. 2002), but suffers from serious drawbacks since AAS requires an individual analysis for each element and typically requires several filters to obtain concentrations for a large variety of 
elements. AAS is also a destructive method, an obvious disadvantage if one wishes to perform further analysis on a particular filter or filters.

\subsubsection{Inductively Coupled Plasma - Atomic Emission Spectroscopy (ICP-AES) and} Mass Spectrometry (ICP-MS)

In ICP-AES, an extracted sample is introduced into a plasma. The plasma is an atmosphere of argon gas that has been seeded with free electrons induced by high voltage from a surrounding Tesla coil (Fassel and Kinseley 1974; Baldwin et al. 1994). The high temperature in the plasma excites the valence electrons out of their ground state and upon return of the electrons to their ground state, a photon of light is emitted that is characteristic of the element with the intensity of the light proportional to the concentration of element. Up to 48 elements can be determined simultaneously using small sample volumes with acceptable detection limits (USEPA 1999; Wilson et al. 2002).

ICP-MS analysis utilizes the elemental ions created by the plasma for separation and identification of their masses. ICP-MS suffers from some serious drawbacks; one main disadvantage is due to ion species from the plasma and sample matrix producing a significant background at certain masses, which results in the formation of polyatomic ions that limit the ability of the technique to detect some elements of interest. As with AAS, ICP techniques are destructive and require a sample to be extracted from the filter prior to analysis. Advantages of ICP-MS include detection limits that are about one order of magnitude lower than other elemental analyses, the ability to analyze 60-plus elements simultaneously, including their isotopes, and the instrument can be set up to analyze a wide dynamic range of aerosol concentrations (USEPA 1999; Wilson et al. 2002).

\subsubsection{X-ray Fluorescence $(X R F)$}

In XRF, incident X-rays are directed onto a sample which ejects an inner shell electron from an atom. The ejected electron creates a vacancy that is filled by an electron from a higher energy orbital in the atom, releasing a fluorescent X-ray as the electron drops in energy. This fluorescent X-ray is measured and its energy is characteristic of the emitting element, while the intensity of the X-ray peak is proportional to the number of atoms of that element in the sample (Watson et al. 
1999). Comparison of the resulting spectrum with known standards allows for the calculation of elemental concentrations in the sample (Davy 2007). Advantages of XRF include the ability to determine the concentrations of more than 40 elements, minimal sample preparation and the XRF process is non-destructive (USEPA 1999; Watson et al. 1999). A disadvantage of XRF is that X-rays from low atomic number elements are emitted at very low levels and are strongly absorbed by the filter material and aerosol deposit (Watson et al. 1999).

\subsubsection{Ion Beam Analysis (IBA)}

The term IBA is used to describe a range of elemental analysis techniques based on the use of ion beams generated by particle accelerators, including particle induced X-ray emission (PIXE), particle induced gamma-ray emission (PIGE), Rutherford backscattering analysis (RBA) and particle elastic scattering analysis (PESA) (Davy 2007). IBA is similar to XRF in that IBA is based on the measurement of X-rays and $\gamma$-rays produced by the ejection of an inner shell electron that are characteristic of the emitting element. In the case of IBA, inner shell electrons are ejected by high energy ions, usually protons, in the two to five million electron volts (MeV) range (Cohen 1999). Elemental concentrations are determined by the spectral intensities of the emitted photons. IBA suffers from the same disadvantage as XRF in quantifying lighter elements, but with more specialized techniques lighter elements like H, C and O can be quantified (Maenhaut and Malmqvist 2001). Similar to XRF, IBA techniques are non-destructive and require minimal sample preparation (Trompetter et al. 2005). A recent review of the use of PIXE for characterizing atmospheric aerosols found that PIXE is an excellent and useful analytical technique (Lucarelli et al. 2011).

\subsubsection{Instrumental Neutron Activation Analysis (INAA)}

INAA involves the irradiation of a sample in the core of a nuclear reactor where neutron bombardment transforms many elements into radioactive isotopes. These isotopes emit characteristic gamma rays that allow for their identification, therefore also allowing the identification of their parent elements. The gamma ray intensity is proportional to the amount of parent element present in the sample and 
elemental concentrations can be quantified through the use of known standards (Wilson et al. 2002). Disadvantages of INAA include the need for a nuclear reactor and INAA is not capable of quantifying some abundant species in ambient PM like Si, $\mathrm{Ni}$, Sn and $\mathrm{Pb}$ (Wilson et al. 2002). While INAA is technically non-destructive, the irradiation process makes the filter membrane brittle and radioactive, limiting the usefulness of the sample for further analysis. Advantages of INAA include the ability to quantify a wide range of elements and low detection limits.

With such a wide-range of choices available for the elemental characterization of PM, it is important for scientists to choose a method or methods that will provide them with the widest range of meaningful data possible. Each analysis method provides a balance of advantages and disadvantages and the choice of which technique or techniques to use are often limited by cost, time or availability of instrumentation.

\subsubsection{Ion Chromatography (IC) and Automated Colorimetry (AC)}

The most common ions of atmospheric interest are sulfate, nitrate, ammonium and chloride (Wilson et al. 2002). Several simple ions like $\mathrm{Na}^{+}, \mathrm{Mg}^{2+}, \mathrm{K}^{+}$and $\mathrm{Ca}^{2+}$ can be determined by AAS, but polyatomic ions must be quantified by other methods. To analyze these ions, PM samples must be extracted from the filter in deionized distilled water, followed by filtration to remove any insoluble material. To analyze water-soluble ions, the filter itself is typically made of a hydrophilic material that allows the water used for extraction to fully penetrate the filter and extract the desired species.

In ion chromatography (IC), sample extracts are passed through an ionexchange column that separates ions by retention time for individual quantification, which is usually done by an electroconductivity detector (Chow and Watson 1999). The ions are identified by their retention times and quantified by the conductivity peak area or peak height (Wilson et al. 2002). Advantages of IC include the ability to analyze more than one ion in a single analysis with low detection limits. The IC process can also be automated, allowing for the unattended analysis of as many as 400 samples (Wilson et al. 2002). The main disadvantages of IC include the need for several separation mechanisms, increasing the potential for interference and 
contamination, and the stringent operating conditions required (Chow and Watson 1999; Davy 2007).

Automated colorimetry (AC) applies colorimetric analyses to small sample volumes with automated throughput (Wilson et al. 2002). Ammonium is the most commonly analyzed ion by AC. AC provides high throughput with minimal operator attention and relatively low maintenance and material costs. Typically the choice between AC and IC is dictated by sensitivity, scheduling and cost since both methods have been found to provide comparable results for all ions within experimental errors (Wilson et al. 2002).

\subsubsection{Carbon Analysis Methods}

There are three classes of carbon that are commonly measured in ambient aerosol samples. The first class is organic carbon (OC), consisting of carbon that is made up of organic compounds like PAHs and hydrocarbons. The second class is elemental carbon (EC) or black carbon (BC) and refers to light-absorbing carbon species. Despite the term "elemental", it is not clear to what degree this fraction of carbon is actually graphitic $(\mathrm{C}(0))$ carbon (Jacobson et al. 2000). The third carbon class is carbonate carbon. The amounts of each class present in a sample depend on the sources contributing to the sample. In Dar es Salaam, Tanzania, $\mathrm{PM}_{10}$ mass was found to consist of $22-24 \%$ OC and 7 - $9 \%$ EC, while the $\mathrm{PM}_{2.5}$ mass was found to consist of $32-38$ \% OC and 14 - 20 \% EC (Mkoma et al. 2010). In the Pearl River Delta Region, China, $\mathrm{PM}_{10}$ mass was found to contain $18 \%$ OC and 7 \% EC on average and the $\mathrm{PM}_{2.5}$ mass was made up of $20 \%$ OC and $8 \%$ EC on average (Cao et al. 2003). $\mathrm{PM}_{10}$ in urban areas of Europe has been found to consist of $30-40 \%$ OC and 5 - $10 \%$ EC, with $\mathrm{PM}_{2.5}$ consisting of $40-50 \%$ OC and $10-20 \%$ EC (Salma et al. 2004; Viana et al. 2006). While each of these regions has their own unique sources of OC and EC, which is reflected in the variations in the relative amounts of OC and EC, they will also have common sources like automobile emissions and biomass burning. In New Zealand, OC and EC have been found to contribute $74 \%$ and $14 \%$ respectively to the $\mathrm{PM}_{2.5}$ mass during peak air pollution events in Christchurch (Scott 2006), and carbonaceous materials have been found to contribute roughly $70 \%$ and $30 \%$ to $\mathrm{PM}_{10}$ mass in Christchurch and Auckland respectively during the winter (Wang et al. 2005a). 
Despite the relatively simple general distinction between OC and EC, in practice the definitions of OC and EC are operational and reflect the method and purpose of the measurement. There are three commonly used thermal methods for the determination of OC and EC in aerosol samples; thermal/optical reflectance (TOR), thermal/optical transmission (TOT) and thermal manganese oxidation (TOM). Each of these methods will be discussed below.

The TOR method was developed in 1982 and has been adapted, with minor variations, by a number of laboratories for the quantification of OC and EC in samples (Huntzicker et al. 1982). In the most commonly applied version of the TOR method (Chow et al. 1993), a filter is subjected to volatilization in a pure helium atmosphere at temperatures ranging from ambient to $550^{\circ} \mathrm{C}$, followed by combustion at temperatures between 550 and $800^{\circ} \mathrm{C}$ in a $2 \%$ oxygen and $98 \%$ helium atmosphere with several ramping stages. The carbonaceous compounds that are evolved at each stage are converted to methane and quantified by a flame ionization detector (FID). The reflectance from the side of the filter that contains the deposit is monitored throughout the analysis. The reflectance typically decreases during volatilization, owing to pyrolysis of the organic material. Upon addition of oxygen, the reflectance increases as light absorbing carbon is combusted and removed. In this method, OC is defined as the fraction of carbon that evolves prior to re-attainment of the original reflectance, with EC defined as the fraction that evolves after the original reflectance has been obtained. It is important to note that by this definition, OC is actually carbon species that do not absorb light at the wavelength used (632.8 nm), and EC is defined as the carbon species that are light absorbing (Chow et al. 1993).

The TOT method applies the same thermal/optical analysis method as the TOR method, but instead of measuring the reflectance, transmission through the filter is measured (Wilson et al. 2002). The IMPROVE_A protocol (Chow et al. 2007a) is now the most commonly used method for determining OC and EC and reports both reflectance and transmittance.

The TMO method relies on manganese dioxide that is in contact with the sample as an oxidizing agent (Fung 1990). In this method, temperature is used to distinguish between OC and EC. Carbonaceous compounds that evolve at $525^{\circ} \mathrm{C}$ are classified as $\mathrm{OC}$ and carbon compounds that evolve at $850^{\circ} \mathrm{C}$ are classified as EC.

Measurement of BC on filters is performed by light reflection or transmission. In the atmosphere, $\mathrm{BC}$ is the most highly absorbing component in the visible spectrum 
with minor absorption contributions from soils, sulfates and nitrates (Horvath 1993; Horvath 1997). To the first order it can be assumed that all light absorption on filters is due to the presence of black carbon. BC is sometimes also referred to as soot in the literature, due to its method of production, namely through combustion processes. Optical methods for the detection of BC are non-destructive and can allow near realtime operation.

A number of studies have been performed to analyze the validity of the terms "elemental carbon" and "black carbon”, with the consensus being that both terms are poor and ill-defined (Andreae and Gelencśer 2006; Bond and Bergstrom 2006). The definition of EC is poor because not all light-absorbing species are graphitic; some organic species like motor oil, tar and coffee also absorb light. Also, a substance of three-bond ( $\mathrm{sp}^{2}$ hybridized) carbon molecules like pencil lead is black and completely absorbs light, while four-bond ( $\mathrm{sp}^{3}$ hybridized) carbon in a diamond is completely transparent and absorbs very little light. Both of these are elemental carbon. The definition of BC is also poor, since there are at least two different classes of organic material that contribute to the absorption of visible light, termed light-absorbing yellow or brown carbon (Andreae and Gelencśer 2006). Despite their rather poor descriptive characteristics, the terms EC and BC continue to be used in the literature and their usage will likely continue in the future.

Carbon isotope analysis has been used to distinguish sources of carbonaceous aerosols. This type of analysis uses ratios of ${ }^{13} \mathrm{C}$ and ${ }^{14} \mathrm{C}$ to the most common isotope of carbon ${ }^{12} \mathrm{C}$. Differences in the ${ }^{13} \mathrm{C} /{ }^{12} \mathrm{C}\left(\delta^{13} \mathrm{C}\right)$ ratio are due to chemical fractionation processes like photosynthesis and atmospheric oxidation. ${ }^{14} \mathrm{C}$, on the other hand, is radioactive, so its presence in the atmosphere is a function of age. ${ }^{14} \mathrm{C}$ is generated in the upper atmosphere by cosmic radiation and is subsequently mixed into the troposphere. Contemporary sources of carbon, like non-fossil fuel carbon and vegetation, have ${ }^{14} \mathrm{C}$ content comparable to the atmospheric concentration at the time it was formed; making ${ }^{14} \mathrm{C}$ a unique tracer for vegetative emissions. Carbon that is released through the combustion of fossil fuels has almost no ${ }^{14} \mathrm{C}$ because it is much older than the half-life of 5726 years.

A study of the ${ }^{14} \mathrm{C}$ content in ambient air samples in Launceston, Tasmania found that 97 - $99 \%$ of the non-carbonate carbon mass during the winter was due to wood smoke from domestic heating appliances and that the combustion of fossil fuels 
contributed a consistent mass of carbon to ambient concentrations year-round (Jordan et al. 2006).

The most commonly used method for the analysis of the speciation of organic compounds from ambient air samples is gas chromatography - mass spectrometry (GC/MS). The reason for the widespread use of this method is that GC can be used to separate a wide range of compounds on a single column and the GC is very easily coupled to a mass spectrometer, making organic compound identification simple and routine (Jacobson et al. 2000). Typically, filter samples are extracted into a solvent and injected into the GC column. The GC/MS method does have some limitations though; in particular, polar solvents cannot be introduced into the GC column, preventing the most polar constituents of a sample from being extracted and polar compounds, like carboxylic acids, that do dissolve in certain non-polar media may bind so strongly to the GC column that they may not elute or may not be sufficiently volatile (Jacobson et al. 2000). As a result of these limitations, only a fraction of the organic material on a filter may be analyzed by GC/MS.

As a way to avoid the tedious process of extracting OC from filters (using a Soxhlet apparatus for an extended time period) a process called thermal desorption has been introduced to efficiently remove OC. In the thermal desorption process, a small sample of the filter is heated in an oven to $300{ }^{\circ} \mathrm{C}$ for a short period of time and the desorbed organic species are condensed into a cryotrap. Once the desorption process is complete, the condensed organic species are rapidly heated and released to the GC column. Thermal desorption has been shown to provide comparable results to the USEPA method TO - 13A in the analysis of NIST reference materials (Waterman et al. 2000; Falkovich and Rudich 2001; Bates et al. 2008). More detailed information about thermal desorption GC/MS can be found in two reviews (Chow et al. 2007b; Hays and Lavrich 2007).

Numerous studies have been performed on the organic speciation of aerosols, with a particular emphasis on combustion processes like wood burning and motor vehicle fuel combustion (Fraser et al. 1999; Fine et al. 2002; Fraser et al. 2002). The combustion process results in a complex mixture of volatile and particle phase OC, formed due to partial combustion and high temperature oxidation (Simoneit 1999). Studies focused on OC emissions from various sources have allowed the identification of "tracer" compounds that are useful in the identification of the sources contributing to ambient PM. Examples of organic tracers commonly used include the 
PAH retene as a tracer for wood combustion (Ramdahl 1983), levoglucosan, a product of the breakdown of cellulose, as a marker for biomass combustion (Simoneit et al. 1998) and the PAH benzo[a]pyrene as a tracer for motor vehicles (Menichini 1992). GC/MS is very useful in quantifying PAHs in the atmosphere. The determination of PAH concentrations in the atmosphere, particularly in urban areas, is important since many are known or suspected of being carcinogens or mutagens (IARC 1998).

As an alternative to GC/MS, liquid chromatography-mass spectrometry (LC/MS) can be used for the characterization of OC. LC/MS is a far less common technique for the analysis of aerosols due to the need for a wide range of columns, many of which are designed to separate analytes within a narrow class of compounds (Jacobson et al. 2000). Since it is desirable to identify a wide variety of compounds, LC/MS is not often the most convenient route for OC characterization. LC does have two major advantages to GC, however. First, aqueous solutions can be injected directly into the column, and secondly, polar compounds do not need to go through a derivitization step in order to elute from most LC columns (Jacobson et al. 2000). Due to the convenience of GC/MS, it is probably best to consider LC/MS as a useful complementary method in order to account for limitations in GC/MS capability.

\subsubsection{Scanning Electron Microscopy - Energy Dispersive Spectroscopy (SEM-EDS)}

SEM-EDS can be used to provide information on single particle size, composition and morphology (Breed et al. 2002; Li et al. 2003; Moreno et al. 2003). SEM utilizes an electron beam to image samples through the scattering of electrons in a process similar to the way images are obtained by optical microscopes through the use of photons. Similarly to PIXE, SEM-EDS utilizes the interaction of electrons in an electron beam with a sample. The collisions of these electrons with the electrons of an atom cause the ejection of an inner shell electron of the atom and the subsequent emission of an X-ray as the atom returns to its ground state. The X-rays emitted by the sample are characteristic of the elements present in the sample. SEM-EDS has a lower sensitivity than IBA analyses due to the lower energy of the incident electrons $(10-20 \mathrm{keV})$. 


\section{Atmospheric Aerosols - Source Apportionment}

The term source apportionment refers to a variety of techniques that attempt to apportion the contributions of individual sources to ambient PM concentrations. The apportionment of sources is a key tool used in the assessment and management of air quality. There are three main categories of source apportionment methods available:

- Emission inventories

- Dispersion modeling

- $\quad$ Receptor modeling

Generally a combination of methods provides the most useful avenue to gaining a meaningful understanding of the relative contributions of various sources to ambient PM. This is particularly important in regards to human health and situations where regulatory action is being considered (Davy 2007).

\subsection{Emissions Inventories}

Emissions inventories are regarded as essential tools for evaluating, managing and regulating air pollution (Mobley and Cadle 2004). Emissions inventories provide measured data or calculated estimates of pollutants emanating from transportation, industrial, biogenic or other anthropogenic sources (Mobley and Cadle 2004). An emissions inventory provides information on the emission of pollutants from known sources on both local and national levels. As such, many countries, including New Zealand, the United States and the UK, have compiled national emissions inventories for various pollutants including $\mathrm{PM}_{10}$, PAHs, dioxins and greenhouse gases (Ministry for the Environment 2001; USEPA 2009; MacCarthy et al. 2010).

The process of establishing an emissions inventory involves gathering source activity data for the sources which may be of interest in a region. This data comes in the form of kilograms of wood burned, vehicle kilometers traveled by motor vehicles and tonnes of raw material processed, for example. This activity data is then multiplied by an emission factor that is specific to the pollutant being investigated and the source being considered, for example grams of pollutant per kilogram of wood burned in the case of domestic heating or grams of pollutant per kilometer traveled for motor vehicles. The process of multiplying source-specific emission factors by source 
activity data is performed for all pollutants of interest in a region or country to establish a "complete" emissions inventory.

Obviously, the value of an emissions inventory is limited by the availability and quality of data that is used to establish the inventory. Some estimates of emissions can be very accurate, for example industrial activities, where stack emissions can be measured directly and correlated very easily with production activity (Davy 2007). For other sources, like motor vehicles and domestic wood burning, emissions estimates require an averaging process in their calculation, limiting their accuracy. Emissions inventories suffer from many uncertainties as a result of the need to obtain information from a wide range of sources and the use of approximations and empirical models in their compilation (Mobley and Cadle 2004). Another drawback of emissions inventories is their resolution. Typically the output from emissions inventories is on a tonnes of pollutant per year basis, although this can be improved slightly to a tonnes of pollutant per average winter/summer day with a consequent increase in the cost of compiling the inventory (Davy 2007).

Perhaps the main drawback of emission inventories is their inability to account for long-range transport of pollutants, formation of secondary aerosols and biogenic sources like marine aerosol (Mobley and Cadle 2004). These factors can all have a significant impact on local air quality. Also, emissions inventories are unable to provide definitive evidence of the primary pollutants responsible for an air pollution episode, information that is relevant where health standards are being exceeded.

\subsection{Dispersion Modeling}

Dispersion, or source modeling, begins by quantifying pollutant emission rates from a source, followed by the application of a mathematical model that accounts for meteorology, chemical transformations and deposition to determine the downwind concentrations of a pollutant at a receptor site (Watson et al. 2002b). Theoretically, ambient concentrations of a pollutant can be calculated and the relative contributions of various sources determined using source modeling, provided that all sources and emission rates for pollutants are known. However, the complexity of the atmosphere, its vertical structure and interaction with topographical elements requires the simplification of the physical and chemical properties included in the dispersion model (Hopke 1999a). The addition of multiple sources to the equations makes 
modeling an entire urban area very difficult without significant simplification. Dispersion modeling is routinely used to assess the downwind impacts of individual sources, but in general, it should be regarded as a bridge between emissions inventories, discussed in Chapter 3.1, and receptor models, discussed in Chapter 3.3.

\subsection{Receptor Modeling}

Receptor models utilize ambient measurements of pollutants of interest to determine sources and the contribution of those sources to ambient concentrations of the pollutant. In general, receptor models are based on the conservation of mass of a pollutant from the point of emission to the point of sampling (Watson 1984; Hopke 1999a). The mathematical formulations of receptor models express ambient chemical concentrations as the sum of the product of species abundances in source emissions and source contributions, or more simply, the measured chemical profile is resolved mathematically to be the sum of a number of different sources (Davy 2007). Receptor models based on the conservation of mass are simplifications and rely on some general assumptions:

- Source emission compositions are constant over the period of ambient and source sampling

- Chemical species do not react with each other, therefore they add linearly

- All source contributions have been identified and their emissions characterized

- The number of sources is less than or equal to the number of species measured

- Source profiles are linearly independent of each other

- Measurement uncertainties are random, uncorrelated and normally distributed The effects of deviations from these assumptions are testable, so the accuracy of source quantification can be evaluated. Uncertainties in the inputted data can also be propagated to evaluate the uncertainty of source contribution estimates.

To illustrate the chemical mass balance approach we can consider the concentration of sodium measured at an urban New Zealand monitoring site. The concentration of sodium at a site like this would contain contributions from sources like domestic wood burning, motor vehicle emissions, soil and marine aerosol, and can be written as (adapted from (Hopke 1999a)):

$$
\mathrm{Na}_{\text {amb }}=\mathrm{Na}_{\text {fire }}+\mathrm{Na}_{\text {marine }}+\mathrm{Na}_{\text {soil }}+\mathrm{Na}_{\text {motorvehicle }}+\ldots
$$


The contribution of an individual source to the ambient concentration of sodium, like sodium due to marine aerosol, can be determined by considering the ambient concentration of sodium due to marine aerosol as the product of the fraction

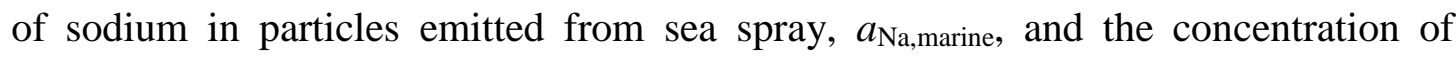
particles of marine aerosol in the air, $f_{\text {marine: }}$

$$
\mathrm{Na}_{\text {marine }}=a_{\mathrm{Na} \text {,marine }} f_{\text {marine }}
$$

Since normally it is desirable to determine a large number of chemical constituents, the chemical mass balance can be extended to account for all $m$ elements in the $n$ samples as contributions from $p$ independent sources

$$
x_{i j}=\sum_{k=1}^{p} a_{i k} f_{k j} \quad i=1, \mathrm{~m}, j=1, \mathrm{n}
$$

where:

$x_{i j}$ - is the $i$ th elemental concentration measured in the $j$ th sample, $a_{i k}-$ is the fraction of the ith element in the material from the $k$ th source, $f_{k j}$ - is the airborne mass concentration of material from the $k$ th source contributing to the $j$ th sample.

When developing a model for identifying and apportioning sources of ambient particle mass, there are a number of physical constraints that must be considered (Hopke 1999a). These constraints are:

(1) The model must explain the observations.

(2) The predicted source compositions must be non-negative.

(3) The predicted source contributions to the aerosol must be non-negative.

(4) The sum of the predicted elemental mass contributions for each source must be less than or equal to the total measured mass for each element.

While it is important to keep these constraints in mind, there are also a number of other factors that affect particle composition from a source and that source's 
contribution to ambient PM (Hopke 1999a). First, composition of particles from a source can vary over time. Using domestic heating as an example, changes in elemental and PAH concentrations occur depending on the type of wood burned and the setting used for wood burner operation (Ancelet et al. 2010). Secondly, the composition of aerosols in the atmosphere is modified through a multitude of processes, some of which are discussed in Chapter 1.2. Thirdly, analytical noise in the measurement process affects limits of detection and introduces analyte intereferences.

Methodologies of three main receptor modeling approaches based on the conservation of mass are discussed below.

\subsubsection{Chemical Mass Balance (CMB)}

The chemical mass balance method solves Equation (3) directly for each sample, assuming that the number of sources and their compositions at the receptor site are known (Hopke 1999a). The composition of an ambient sample is then used in a multiple linear regression against source compositions to derive the mass contribution of each source to the particular sample. Originally the CMB problem was solved using ordinary least squares (Friedlander 1973), but since then the method of effective variance weighting has become the approach that is used more commonly (Watson et al. 1984). Effective variance weighting includes uncertainties in the measurement of source composition profiles as well as uncertainties in ambient measurements. A method was also developed to permit the calculation of uncertainties in the mass contributions. The USEPA has developed software incorporating effective variance least squares to solve the CMB equation using a standard personal computer. The main disadvantages of CMB are that representative source profiles are required, but not always available and CMB assumes that all observed mass is due to the sources selected in advance by the person performing the analysis, introducing subjectivity into the analysis.

\subsubsection{Principal Components Analysis (PCA)}

PCA is a form of eigenvector analysis that tries to simplify the description of a system by determining a minimum set of basis vectors that span the data space to be interpreted (Hopke 1999a). In other words, a new set of variables are found as linear combinations of the measured variables so that the observed variations in the system 
can be reproduced by a smaller number of causal factors. PCA can only be performed on a set of samples in which various sources contribute different amounts of particles to each sample, so the mass balance equation must be expanded to a matrix equation:

$$
\mathrm{Z}=\mathrm{A} \cdot \mathrm{F}
$$

where:

$\mathrm{Z}$ - is a matrix of sample vectors

A - is the matrix of loading vectors related to source compositions

$\mathrm{F}$ - is the matrix of scores related to the contribution of a source type to the variance of the particular measured variable

PCA can identify major source types and relate secondary components to sources via correlations and is sensitive to the influence of unknown or minor sources. Disadvantages of the PCA method include sensitivity to extreme values and the vectors are usually related to broad source types instead of specific sources.

\subsubsection{Positive Matrix Factorization (PMF)}

Positive matrix factorization was introduced by Paatero and Tapper in 1993 (Paatero and Tapper 1993). PMF assumes that ambient concentrations can be explained by the linear product of a source matrix and contribution matrix. These matrices are obtained by an iterative minimization process, with a non-negativity constraint built into the model so that source contributions and profile abundances are always greater than zero. Selected factors can then be interpreted as source profiles by comparison with source measurements. PMF has many advantages including available software, the ability to incorporate uncertainties and missing or below detection limit data, the ability to weight species concentrations by their analytical precision and source profiles can be derived from ambient measurements as they would appear at the receptor. The main disadvantages of PMF are that large data sets are required, source profiles are required to verify the representativeness of calculated profiles, PMF is limited to the accuracy, precision and species measured at the monitoring site and PMF relies on a number of parameters and initial conditions as model input, making it sensitive to pre-set parameters (Davy 2007). 


\section{A review of air particulate matter research in New Zealand: Where are we and where should we go from here?}

\subsection{Synopsis}

This chapter has been reproduced in full, with permission, as it appears in Air Quality and Climate Change, 2012. Ancelet, T., Davy, P. K., Trompetter, W. J., Markwitz, A. and Weatherburn, D. C., 2012. A review of air particulate matter research in New Zealand: Where are we and where should we go from here? Air Quality and Climate Change, 46, 34-42. *Note*: The HAPiNZ report has been updated since this work was published http://www.hapinz.org.nz/HAPINZ\%20Update_Vol\%201\%20Summary\%20Report.p df.

This review was intended to provide an overview of PM research that has been undertaken in New Zealand. From this review it is evident that many urban areas in New Zealand suffer episodes of poor air quality during the winter as a result of PM. Furthermore, domestic heating emissions from wood burning were identified as the dominant source of PM during the winter throughout the country. This work also highlighted that concentrations of potentially harmful species like benzo[a]pyrene and arsenic are elevated during the winter in many areas, to the extent that these species may be exceeding national guidelines. Suggestions for further study were also provided, with an emphasis on better understanding long-term trends in PM pollution and its components.

\subsection{Introduction}

Particulate matter (PM) in the atmosphere is made up of a wide range of sizes, from diameters of a few nanometers to around $100 \mu \mathrm{m}$. A number of excellent reviews focusing on the various properties, including the formation, growth and transformation of PM are available (Jacobson et al. 2000; Pöschl 2005; Colbeck and Lazaridis 2010). Typically there are two particle metrics used for the purposes of monitoring and regulation, $\mathrm{PM}_{10}$ and $\mathrm{PM}_{2.5}$. $\mathrm{PM}_{10}$ is defined as those particles with aerodynamic diameters less than $10 \mu \mathrm{m}$, while $\mathrm{PM}_{2.5}$ is similarly defined and refers to 
particles smaller than $2.5 \mu \mathrm{m}$. $\mathrm{PM}_{2.5}$ is often also referred to as the fine fraction. The coarse fraction of $\mathrm{PM}_{10}$ is made up of particles with diameters in the 2.5-10 $\mu \mathrm{m}$ size range and is denoted as $\mathrm{PM}_{10-2.5}$. Unless explicitly stated, these definitions will be used throughout this work.

The adverse effects of PM on human health and the environment are wellknown (Dockery et al. 1993; Tsai and Cheng 1999; Nel 2005; Russell and Brunekreef 2009; Pósfai and Buseck 2010). While most of New Zealand generally has clean air for much of the year, local studies have reported adverse health effects resulting from exposure to PM (Epton et al. 2008; Howden-Chapman et al. 2008; Murdoch and Jennings 2009; Hales et al. 2010; Fukuda et al. 2011). The references provided are not intended to be exhaustive, as a full review of health effects associated with PM in New Zealand is outside the scope of this work, but are intended to highlight recent studies that show the diverse effects that PM has on the health of New Zealanders.

A study led by the National Institute of Water and Atmospheric Research (NIWA) found that $\mathrm{PM}_{10}$ emissions from motor vehicles in New Zealand likely resulted in 399 premature deaths each year, with a total of 970 premature deaths associated with $\mathrm{PM}_{10}$ pollution (Fisher et al. 2002). After this initial work, the Health and Air Pollution in New Zealand (HAPiNZ) study was commissioned to determine the environmental, health, social and economic costs of air pollution from all sources throughout New Zealand (Fisher et al. 2007). The HAPiNZ study estimated that $\mathrm{PM}_{10}$ contributed to approximately 900 premature deaths each year and was the most significant contributor to adverse health effects like bronchitis and acute respiratory and cardiac conditions. The study also suggested that anthropogenic $\mathrm{PM}_{10}$ pollution resulted in almost 2 million restricted-activity days. From an economic standpoint, the HAPiNZ study estimated the total cost of air pollution to be $\$ 1.3$ billion per year, when hospital admissions and restricted-activity days were included in the calculations. The most recent update of the HAPiNZ study suggested that $\mathrm{PM}_{10}$ emissions were the most significant contributor to the more than 1600 premature deaths resulting from air pollution (Kuschel and Mahon 2010).

Based on the significant adverse health effects and costs due to PM pollution, this work attempts to provide an overview on the current understanding of PM in New Zealand. Not surprisingly, a significant fraction of this research has focused on New Zealand's larger urban centers (Auckland and Christchurch), but a number of studies 
do exist for smaller towns and cities. Future implications and potential research opportunities are also presented and discussed.

It should also be noted that this paper is largely focused on research that has been published in peer-reviewed journals and has not necessarily accounted for each internal report or study that make up the 'grey' literature in New Zealand. This aspect will be discussed later in the manuscript.

\subsection{Regulatory Framework}

The management of air quality in New Zealand is governed by the Resource Management Act 1991 (RMA) and in October 2004 the New Zealand government introduced the National Environmental Standards for Air Quality (NESAQ). At their introduction, the NESAQ included: seven standards banning activities that discharge significant quantities of dioxins and other toxics, five ambient air quality standards (for $\mathrm{CO}, \mathrm{PM}_{10}, \mathrm{NO}_{2}, \mathrm{SO}_{2}$ and $\mathrm{O}_{3}$ ), a design standard for new woodburners installed in urban areas and a requirement for landfills with over 1 million tonnes of refuse to collect greenhouse gas emissions. The NESAQ prescribes a mean daily maximum concentration of $50 \mu \mathrm{g} \mathrm{m}^{-3}$ for $\mathrm{PM}_{10}$, which initially was not to be exceeded more than once per year by 2013. Regions breaching the NESAQ after 2013 would have been forced to decline all 'discharge-to-air' permits for emissions containing $\mathrm{PM}_{10}$. The NESAQ have since been revised based on a number of specific concerns regarding perceived stringency of the NESAQ, issues regarding inequity and inabilities in meeting the original timeframes (Ministry for the Environment 2011). The revised standards include an extension of the target date for regional councils to meet the ambient $\mathrm{PM}_{10}$ standard. The new target dates are 1 September 2016 for airsheds with between 1 and 10 exceedances of the standard and 1 September 2020 for airsheds with 10 or more exceedances. Also included are a provision for excluding exceptional events such as dust storms and volcanic eruptions, a requirement for 'offsets' from certain new industries with $\mathrm{PM}_{10}$ discharges in 'polluted' airsheds from September 2012 and from September 2012, new domestic solid-fuel burning open fires will be prohibited in polluted airsheds. Because the NESAQ are intended to provide a minimum acceptable safeguard for New Zealand's population, it is also possible for regional councils to impose stricter regulations locally. 
Alongside the NESAQ, there are health-based Ambient Air Quality Guidelines (AAQG) for 15 pollutants (Ministry for the Environment 2002b). These pollutants include: $\mathrm{CO}, \mathrm{PM}_{10}, \mathrm{NO}_{2}, \mathrm{SO}_{2}, \mathrm{O}_{3}, \mathrm{~Pb}, \mathrm{H}_{2} \mathrm{~S}$, acetaldehyde, benzene, 1,3-butadiene, formaldehyde, benzo(a)pyrene, $\mathrm{Hg}$, $\mathrm{Cr}$ and As. In preparing the NESAQ, the 24-hour average AAQG for $\mathrm{PM}_{10}$ of $50 \mu \mathrm{g} \mathrm{m}^{-3}$ was used and the AAQG also proposes an annual average $\mathrm{PM}_{10}$ concentration not exceeding $20 \mu \mathrm{g} \mathrm{m} \mathrm{m}^{-3}$. The AAQG recommends a monitoring value of $25 \mu \mathrm{g} \mathrm{m}^{-3}$ (24-hour average) for $\mathrm{PM}_{2.5}$ to be used for assessing monitoring results and to judge whether further investigations are needed to quantify $\mathrm{PM}_{2.5}$ sources. While some of the pollutants with AAQGs are routinely monitored by regional councils, many are not, based on cost considerations.

\section{4 $\quad$ PM $_{10}$ Concentrations Throughout New Zealand}

During the summer, $\mathrm{PM}_{10}$ concentrations throughout New Zealand tend to be low and are largely influenced by natural sources like marine aerosol and soil, particularly outside of Auckland. This is not the case, however, during the winter when $\mathrm{PM}_{10}$ concentrations regularly exceed the NESAQ in a number of regions. In a study of $\mathrm{PM}_{10}$ monitoring data from a number of New Zealand urban environments, Trompetter et al. (2010) showed that $\mathrm{PM}_{10}$ has distinct diurnal cycles, independent of community size or population. Peak $\mathrm{PM}_{10}$ concentrations were found to occur between 22:00 and midnight with a second, less pronounced peak also occurring between 8:00-11:00 am (Trompetter et al. 2010). Figure 4.1 presents a plot of the hourly average $\mathrm{PM}_{10}$ concentrations in each of the urban areas studied. Trompetter et al. (2010) determined that environmental confinement resulting from the development of stable inversion layers coupled with emissions from domestic fires was the most significant reason for the build-up of $\mathrm{PM}_{10}$ in urban areas. 


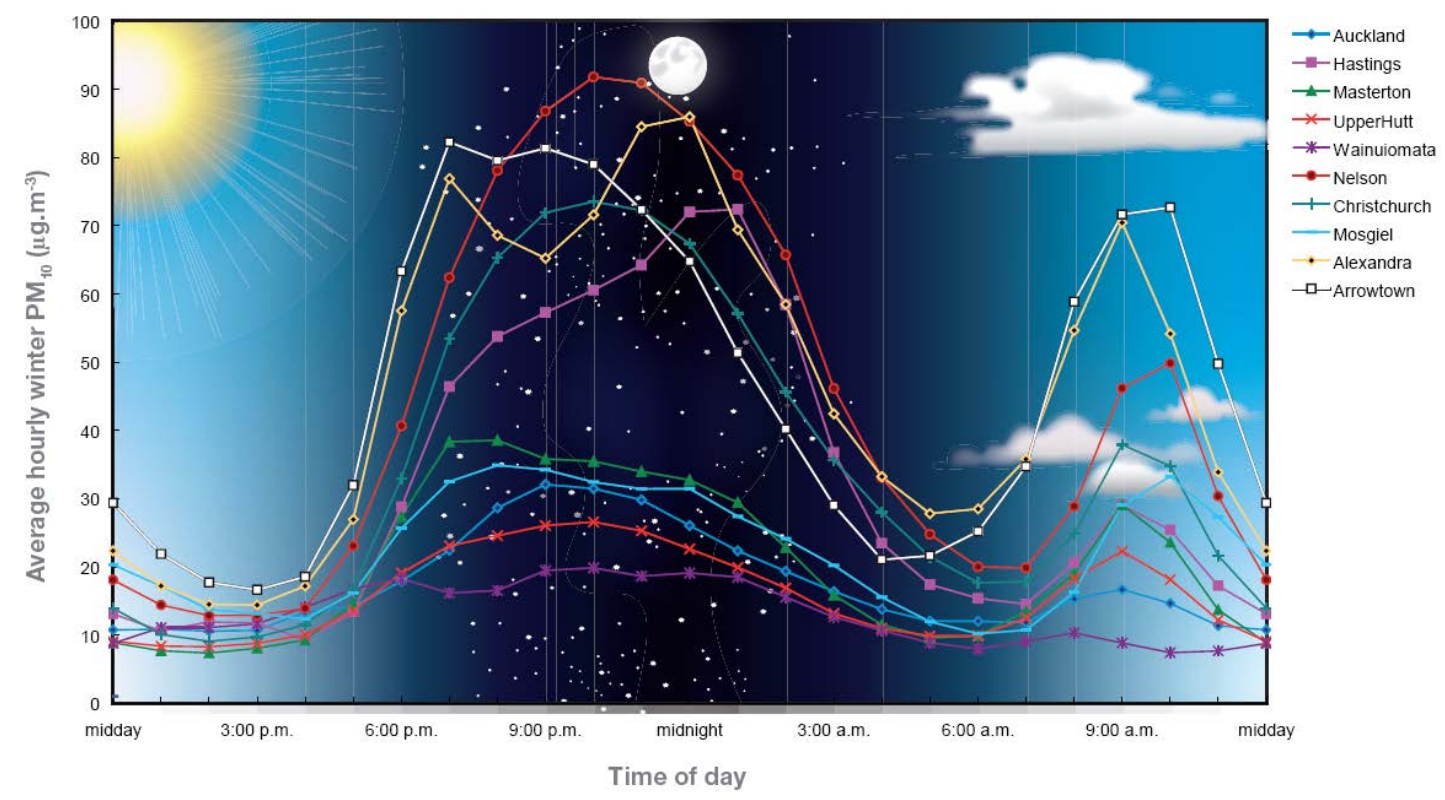

Figure 4.1 Average hourly winter $\mathrm{PM}_{10}$ concentrations for ten urban areas across New Zealand (adapted from Trompetter et al., 2010).

In addition to the report from Trompetter et al. (2010), a nationwide study by Pearce and Kingham (2008) found that residents of low income communities or neighbourhoods, along with other features associated with socioeconomic status, tended to have higher exposure to elevated $\mathrm{PM}_{10}$ concentrations. They suggested that this uneven exposure plays an important role in the disparate health status among low income communities in New Zealand (Pearce and Kingham 2008). Overall, these studies provide relevant and highly useful information about $\mathrm{PM}_{10}$ concentrations and patterns throughout New Zealand.

The regulatory framework for air quality management in New Zealand is centered on managing pollutant concentrations within designated airsheds to comply with the NESAQ. Effective management therefore necessitates an understanding of the sources of pollutants and the contribution from each source to overall pollutant loadings which, for particulate matter, is a driver for compositional research (e.g. chemical, particle size and particle number). For more detailed information about $\mathrm{PM}_{10}$ monitoring in specific airsheds, readers are encouraged to consult the New Zealand Ministry for the Environment website (www.mfe.govt.nz) or the websites of the respective regional and unitary councils.

The following sections describe PM compositional research undertaken for New Zealand urban areas. Auckland and Christchurch are mentioned specifically due 
to the number and variety of studies for each of the cities. The research has been divided into subsections based on the intent of the research and the nature of species studied.

\subsection{Auckland}

As New Zealand's largest city, Auckland (1.3 million people) has been the focus of a significant amount of wide-ranging PM research. As it is located on a narrow isthmus, generally Auckland is well-ventilated, but some areas are subject to the formation of localized micro-climates that can significantly elevate $\mathrm{PM}_{10}$ concentrations for a number of days (Sparrow 1968; Longley and Harper 2010). Early accounts of air pollution in Auckland and the establishment of the Auckland Air Pollution Research Committee have been reported (Sparrow et al. 1969; Fitzgerald et al. 1973).

\subsubsection{Ionic Species}

Water soluble inorganic species in Auckland were characterized in three separate studies (Wang and Shooter 2001; Wang and Shooter 2002; Wang et al. 2005b). Wang and Shooter (2001) characterized eight soluble ions in $\mathrm{PM}_{10}$ and found significant seasonal variations in $\mathrm{Na}^{+}, \mathrm{Cl}^{-}$and $\mathrm{SO}_{4}{ }^{2-}$ concentrations, with peak concentrations occurring during the summer. This feature was attributed to an increased marine influence during the summer. Wang and Shooter (2002) also examined fine/coarse and day/night variations for eight water soluble ions in Auckland during the winter. They found that $\mathrm{PM}_{10}$ concentrations did not have a pronounced day/night difference, but concentrations of $\mathrm{SO}_{4}{ }^{2-}$ and $\mathrm{NO}_{3}{ }^{-}$increased during the day and night, respectively. A clear difference was observed in the compositions of size-segregated samples. Coarse samples were enriched in sea salt ions $\left(\mathrm{Na}^{+}, \mathrm{Mg}^{2+}\right.$ and $\left.\mathrm{Cl}^{-}\right)$while fine samples were enriched with non-sea salt ions $\left(\mathrm{SO}_{4}{ }^{2-}\right.$ and $\left.\mathrm{NO}_{3}{ }^{-}\right)$. In a later study, Wang et al. (2005) determined the concentrations of different carbonaceous and ionic components in $\mathrm{PM}_{10}$ during the winter in Auckland. Using principal components analysis (PCA) they suggested that marine aerosol was the most significant contributor to $\mathrm{PM}_{10}$ concentrations followed by vehicular emissions. It is worth noting that in all three manuscripts the authors 
suggested that contributions to $\mathrm{PM}_{10}$ from domestic heating were insignificant, based on low concentrations of non-sea salt (nss) potassium.

\subsubsection{Carbonaceous Species}

Carbonaceous species in the atmosphere are primarily composed of organic compounds, such as polycyclic aromatic hydrocarbons (PAHs) and a multitude of other hydrocarbon compounds (Jacobson et al. 2000), elemental carbon (EC) as a byproduct of incomplete combustion and inorganic carbon compounds (mainly carbonates). Separate studies in different years on the composition of organic pollutants during the winter in Auckland resulted in different conclusions (Krivácsy et al. 2006; Wang et al. 2006). Krivácsy et al. (2006) characterized almost 100 organic compounds from $\mathrm{PM}_{10}$ samples collected in 2001 and found that, during winter, organic species accounted for $21-45 \%$ of $\mathrm{PM}_{10}$. Resin acids, fatty acids, tricyclic terpenoic acids and carbohydrates were the most abundant organics. Dehydroabietic acid, a tracer for biomass burning (Simoneit 2002), was the most abundant organic compound identified, suggesting that domestic wood combustion was the most dominant source of carbonaceous PM. The authors also noted that hopanes and sterans, markers for vehicular emissions (Cass 1998), were not detected. Wang et al. (2006) characterized 69 organic species in $\mathrm{PM}_{10}$ collected during the winter of 2004 and found that levoglucosan and dehydroabietic acid were the most abundant compound and fatty acids were the most abundant class of compounds characterized. They also reported that hopanes were detected in low concentrations $(0.02-0.05 \%$ of the total organic carbon (TOC)). Despite the vast abundance of biomass burning tracers (levoglucosan and dehydroabietic acid), the authors concluded that motor vehicles were the most significant contributor to carbonaceous $\mathrm{PM}_{10}$ in Auckland. It seems apparent from the results of both studies that, contrary to the conclusion of Wang et al. (2006), biomass burning during the winter was the primary source of carbonaceous species in Auckland.

PAHs are pollutants that are of concern because many are known or suspected to be carcinogens and mutagens (IARC 1998). Benzo(a)pyrene (BaP), for example, is classified as a priority pollutant by the Ministry for the Environment and has an

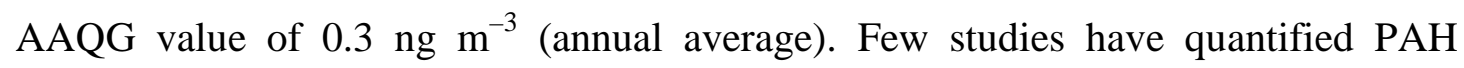
concentrations in Auckland (Khanal and Shooter 2004; Cavanagh et al. 2009). Khanal 
and Shooter (2004) collected $\mathrm{PM}_{10}$ samples over each season and quantified 16 PAHs, with a particular focus on BaP. The median BaP concentrations were 0.27, 0.02, 0.13 and $0.42 \mathrm{ng} \mathrm{m} \mathrm{m}^{-3}$ in spring, summer, autumn and winter, respectively. BaP concentrations correlated well with $\mathrm{PM}_{10}$ and total $\mathrm{PAH}$ concentrations, indicating similar emission sources. Based on their results, the authors calculated the annual average concentration of $\mathrm{BaP}$ to be $0.33 \mathrm{ng} \mathrm{m}^{-3}$. While this value was calculated using $\mathrm{BaP}$ concentrations from a limited number of days (56), it does indicate that $\mathrm{BaP}$ concentrations could be exceeding the AAQG in Auckland. In a portion of their study, Cavanagh et al. (2009) characterized 34 PAHs (16 USEPA priority PAHs and 18 other PAHs) in PM10 from Auckland and determined that the median total concentration of quantified PAHs was $91 \mathrm{ng} \mathrm{mg}^{-1}$ of $\mathrm{PM}_{10}$, with the highest concentrations measured from samples collected during March. The median BaP concentration was $6.24 \mathrm{ng} \mathrm{mg}^{-1}$ of $\mathrm{PM}_{10}$, with peak concentrations occurring in July. Contrary to the results of Khanal and Shooter (2004), PAH concentrations were not correlated with $\mathrm{PM}_{10}$ concentrations.

\subsubsection{Source Apportionment Studies}

Source apportionment studies provide information on the sources of PM and the contributions of the identified sources to PM mass. An investigation of 'brown haze' episodes in Auckland using enrichment factors and PCA concluded that emissions from diesel-fuelled vehicles, and to a lesser extent, coal combustion, were responsible for these events (Senaratne and Shooter 2004). Given the small number of samples collected (9 coarse and fine samples on brown haze days and 5 'normal' days; $6 \mathrm{PM}_{10}$ samples from brown haze days and 4 from 'normal' days), the seemingly arbitrary use of combined particle fractions for selected elements for source apportionment (especially because it was recognized that 'brown haze' events were primarily due to the light scattering properties of fine particles) and the lack of association of black carbon (BC) with the diesel particle source, it seems to us that this study is of limited scientific value and resulted in a number of contradictory statements and conclusions that are difficult to reconcile.

In a larger study, size-resolved samples collected during the summer (48) and winter (47) were collected and analyzed using positive matrix factorization (PMF) (Wang and Shooter 2005). Five factors were extracted for both the fine and coarse 
fractions. The fine fraction sources were identified as: soil (as a combination of road dust and other crustal matter sources), secondary ammonium sulphate, aged sea salt, motor vehicle emissions and industrial emissions/waste incineration. Generally the source profiles and the rationalizations for the assignments appear reasonable, but the two combustion sources could easily represent biomass burning and motor vehicles instead of motor vehicles and industry/incineration, respectively, because of the association of $\mathrm{K}$ with the fourth factor and $\mathrm{Ca}, \mathrm{Fe}$ and $\mathrm{Zn}$ with the fifth factor (Khalil and Rasmussen 2003; Thorpe and Harrison 2008). The coarse fraction sources were identified as: road dust/other crustal matter, wind-blown crustal matter, aged sea salt, sea salt and coarse nitrate. These source profiles also appeared to contain reasonable associations, although the aged sea salt profiles appears to have suffered more from $\mathrm{Na}$ loss than $\mathrm{Cl}$ loss and the appearance of secondary nitrate in the coarse fraction is at odds with the formation processes of gas-to-particle conversion and heterogeneous acid-base chemistry (Colbeck and Lazaridis 2010). Sea salt and motor vehicles were determined to be the predominant sources to both size fractions during both seasons.

A long-term, multi-site source apportionment study commissioned by Auckland Council has been ongoing since 2006 (Davy et al. 2010). During the study almost $5000 \mathrm{PM}_{2.5}$ and $\mathrm{PM}_{10}$ filters were analyzed for elemental content and black carbon (BC). Five common source contributors were identified for $\mathrm{PM}_{2.5}$ and $\mathrm{PM}_{10}$ samples from each site, including biomass burning, motor vehicles, sulphate, marine aerosol and soil or crustal matter. Site-specific sources such as industrial and shipping emissions and roadworks were also identified. The source profiles and assignments are in much better agreement with other receptor modeling studies than those reported by Wang and Shooter (2005). A number of important features have been identified by this study. First, $\mathrm{PM}_{2.5}$ was largely responsible for peak $\mathrm{PM}_{10}$ concentrations during the winter, with motor vehicles and biomass burning being the primary contributors to $\mathrm{PM}_{2.5}$. Peak $\mathrm{PM}_{2.5}$ concentrations were primarily the result of biomass burning. Second, motor vehicles provided consistent mass contributions to $\mathrm{PM}_{2.5}$ and $\mathrm{PM}_{10}$ throughout the year, illustrating that the coarse fraction is an important metric due to motor vehicle contributions to coarse fraction heavy metal concentrations. Third, marine aerosol was responsible for peak $\mathrm{PM}_{10}$ concentrations during the spring and summer, and at times, when coupled with contributions from other sources, may be sufficient to result in exceedences of the NESAQ. The duration of the study has also provided a clearer image of specific sources. For example, shipping emissions were 
identified to have significant local and regional impacts on primary and secondary sulphate concentrations. Elevated arsenic concentrations were also measured during the winter and were found to be associated with biomass burning, most likely the result of copper chrome arsenate (CCA)-treated timber being used as fuel. Elevated arsenic concentrations during the winter were also reported by Cavanagh et al. (2009). Finally, a trans-boundary event due to a dust storm in Australia in September 2009 that resulted in region-wide exceedances of the NESAQ was identified. This study demonstrates the usefulness of long-term PM monitoring for managing PM pollution in Auckland.

\subsection{Christchurch}

Despite having a lower population than Auckland, Christchurch (350 000 people) is well-documented to suffer from degraded air quality during the winter. The poor air quality in Christchurch is the result of two main factors, significant biomass burning (wood and coal) and frequent meteorological conditions conducive to pollutant build-up. The main meteorological factors contributing to elevated $\mathrm{PM}_{10}$ concentrations are low wind speeds, strong shallow surface-based inversions and topographically influenced local wind systems occurring during anticyclonic periods (Spronken-Smith et al. 2001). Nocturnal radiative cooling of the ground leads to the development of cold air drainage from the Canterbury Plains and Port Hills and the convergence of these drainage winds contributes to stagnation of the air over the city (Sturman and Zawar-Reza 2002; Kossmann and Sturman 2004). In a detailed vertical profile study it was found that PM (dominated by the fine fraction) from domestic fires was emitted into a highly stable and shallow katabatic flow layer, giving rise to high PM concentrations that can persist late into the night (McKendry et al. 2004). McKendry et al. (2004) also found that distinct layers of PM formed and undercutting by shallow drainage flows led to the elevation of layers with a daytime surface PM signature above the highly polluted katabatic layer. These mechanisms are also likely to occur in other New Zealand regions.

$\mathrm{PM}_{10}$ concentrations within Christchurch vary, with elevated locations tending to have lower PM10 concentrations as a result of dispersion by the prevailing katabatic flows (Wilson et al. 2006). Elevated $\mathrm{PM}_{10}$ concentrations in Christchurch have also been shown to be responsible for elevated concentrations of $\mathrm{HNO}_{2}$, 
produced through heterogeneous reactions of $\mathrm{NO}_{2}$ on PM surfaces (Reisinger 2000). Recently, the results of residential heating interventions in Christchurch and Timaru have been reported (Scott and Scarrott 2011). The introduction of intervention measures and a residential heater replacement program were found to markedly reduce wintertime $\mathrm{PM}_{10}$ emissions and concentrations in Christchurch. In Timaru, where lesser restrictions were applied, lower impacts on $\mathrm{PM}_{10}$ emissions and concentrations were observed. The study suggests that despite the reductions, there is a low probability of compliance with the NESAQ in Christchurch and much less likelihood of compliance with the NESAQ in Timaru.

Visibility in Christchurch is also a significant concern. It has been reported that PM (through scattering and absorption) accounted for the most significant amount of light extinction (Wilton 2003). Interestingly, PM from biomass burning was not found to be a significant contributor to reduced visibility. Motor vehicle emissions and secondary particles were found to be the largest contributors to reduced visibility.

\subsubsection{Ionic Species}

In their studies, Wang and Shooter (2001, 2002) found elevated concentrations of $\mathrm{NH}_{4}{ }^{+}, \mathrm{K}^{+}$and $\mathrm{SO}_{4}{ }^{2-}$ during the autumn and winter in Christchurch. It was suggested that acidic species and low wind speeds was responsible for the elevated $\mathrm{NH}_{4}{ }^{+}$concentrations and home heating was responsible for elevated concentrations of $\mathrm{K}^{+}$and $\mathrm{SO}_{4}{ }^{2-}$. Like Auckland, ions associated with marine aerosol $\left(\mathrm{Cl}^{-}, \mathrm{Na}^{+}\right.$and $\mathrm{Mg}^{2+}$ ) were abundant, particularly during the summer when home heating is not necessary. Nighttime concentrations of biomass burning tracers (nss- $\mathrm{K}^{+}$and nss- $\mathrm{SO}_{4}{ }^{2-}$ ) were significantly higher than daytime concentrations, while marine aerosol tracers did not show day-night variations. Ions associated with combustion processes were found to be enriched in the fine fraction, while ions associated with crustal matter and marine aerosol were enriched in the coarse fraction. Based on the abundance of biomass burning tracers during the winter, Wang and Shooter suggested that residential heating was the most significant contributor to the elevated $\mathrm{PM}_{10}$ concentrations measured during the winter. 


\subsubsection{Carbonaceous Species}

An early attempt to determine the sources of PAHs in Christchurch found that domestic heating emissions were the principal contributor to elevated concentrations of PAHs, with motor vehicles producing constant, but far lower concentrations of PAHs (Cretney et al. 1985). Cretney et al. (1985) reported total PAH concentrations ranging from $123-871 \mathrm{ng} \mathrm{m}^{-3}$ and $\mathrm{BaP}$ concentrations ranging from 8-72 $\mathrm{ng} \mathrm{m}^{-3}$ during the winter. In a study investigating the threshold and non-threshold toxicities of PM, PAH and BaP concentrations were reported to be higher than those reported by Cretney et al. (1985) during the winter (Brown et al. 2005). Brown et al. (2005) reported average $\mathrm{PAH}$ and $\mathrm{BaP}$ concentrations to be $52.08 \mathrm{ng} \mathrm{m}^{-3}$ and $7.72 \mathrm{ng} \mathrm{m}^{-3}$ during the winter. Cavanagh et al. (2009) reported USEPA criteria PAH concentrations ranging from 5.1-101 $\mathrm{ng} \mathrm{m}^{-3}$ in $\mathrm{PM}_{10}$ and $\mathrm{BaP}$ concentrations ranging from 0.03-12.31 $\mathrm{ng} \mathrm{m}^{-3}$ in $\mathrm{PM}_{10}$. Each study found that PAH concentrations were highly correlated with PM concentrations during the winter, although this correlation was not explicitly stated by Cretney et al. (1985). To determine the exposure of a population to PAHs, urinary 1-hydroxypyrene (1-OHP) was used as a marker for a group of schoolchildren in Christchurch (Cavanagh et al. 2007). The study found that during high pollution events urinary 1-OHP concentrations were significantly elevated compared with low pollution periods. While the highest concentrations of urinary 1-OHP were low by international standards, it is evident that increased PAH concentrations during the winter result in significantly higher exposures.

The studies of Krivácsy et al. (2006) and Wang et al. (2006) discussed in the Auckland carbonaceous species section also focused on Christchurch. Both studies found that, while the main organic species in $\mathrm{PM}_{10}$ from Christchurch were similar, organic compounds made up a much larger fraction of the $\mathrm{PM}_{10}$ mass in Christchurch, indicative of more intensive domestic wood combustion.

\subsubsection{Source Apportionment Studies}

A source apportionment study using enrichment factors, PCA and absolute PCA identified five $\mathrm{PM}_{10}$ sources in Christchurch (Senaratne et al. 2005). The five sources identified were: domestic emissions (biomass burning) (29\%), sea spray (20 $\%$ ), road dust/crustal matter (21\%), diesel and petrol emissions (30\% combined). The authors concluded that the impact of motor vehicles on $\mathrm{PM}_{10}$ concentrations in 
Christchurch needed to be studied further. However, this study suffered from characterizing too few elements in too few samples, making it difficult to identify the sources responsible for each factor produced and therefore the results may be unreliable.

A separate study on the sources of $\mathrm{PM}_{2.5}$ in Christchurch using PMF identified five sources, including biomass burning, marine aerosol, motor vehicles, secondary aerosols and aged aerosols (Scott 2006). Aged aerosols were found to be the most significant $\mathrm{PM}_{2.5}$ contributor during the summer, while biomass burning was by far the most significant $\mathrm{PM}_{2.5}$ source during the winter. The source profiles presented contained acceptable associations and accounted for $92 \%$ of the measured $\mathrm{PM}_{2.5}$ mass on average.

\subsection{Other Locations}

\subsubsection{Hamilton}

A preliminary study aimed at characterizing PM sources in Hamilton (130 000 people) through elemental fingerprinting roughly identified four major sources of PM (marine aerosol, soil, biomass burning and motor vehicles) (Petersen and Fisher 1999). Sea salt was identified to be the most significant contributor to the elemental concentrations measured. The sources identified appear to be accurate based on their elemental compositions, but the study suffered from characterizing only a limited number of samples and species. Since important species like organic carbon were not quantified, the relative importance of combustion sources could not be accounted for.

Wang and Shooter (2001) characterized water soluble ions in Hamilton as part of their wider study that included Auckland and Christchurch. The relative abundances of ionic species in Hamilton were similar to those in Christchurch and Hamilton had the lowest overall concentrations of all the ionic species measured among the three cities.

\subsubsection{Hastings}

A source apportionment study using PMF in Hastings (28 000 people) identified five major $\mathrm{PM}_{10}$ sources (sea spray, domestic heating, sulphate, soil and motor vehicles) (Wilton et al. 2009). The source profiles presented in this study are 
consistent with other studies and biomass burning was found to be the main source of $\mathrm{PM}_{10}$ from April-October, with smaller contributions during the summer. Wilton et al. (2009) also found that on days where the NESAQ was exceeded, contributions from domestic heating increased (from 82 \% (winter average) to $87 \%$ ). Using airshed modeling, the authors found that pollutants released from outside Hastings also contributed to measured $\mathrm{PM}_{10}$ concentrations in Hastings as a result of dispersion.

\subsubsection{Rotorua}

The development of an emissions inventory and an airshed model for Rotorua (58 000 people) were reported in two separate studies (Fisher et al. 2008; Graham and Iremonger 2008). Similar to other locations in New Zealand, Rotorua experiences high concentrations of $\mathrm{PM}_{10}$ during the winter that regularly exceed the NESAQ. The emissions inventory developed identified domestic heating emissions to have a contribution of $60 \%$ during the winter. The emissions inventory also suggested that small industrial and commercial sources were low contributors to overall $\mathrm{PM}_{10}$ concentrations. Through airshed modeling, the authors suggested that a $60 \%$ reduction in $\mathrm{PM}_{10}$ from domestic heating emissions was the optimum strategy to achieve compliance with the NESAQ.

\subsubsection{Greater Wellington Region}

The sources responsible for PM pollution in a number of areas within the Greater Wellington region (450 000 people) have been studied using multi-elemental analyses and PMF (Davy 2007). In Upper Hutt, three coarse (road dust, soil and sea salt) and four fine (sulphate, motor vehicles, wood burning and sea salt) sources were identified. During the winter, biomass burning was the dominant source, followed by motor vehicles. Secondary sulphate was found to be an important contributor to fine particle mass, particularly during the summer. In Seaview, an industrial area adjacent to the mouth of the Hutt River and Wellington Harbour, four coarse (seas salt, road dust, soil and zinc) and five fine (sulphate, motor vehicles, zinc, sea salt and soil) sources were identified. It was suggested that the zinc sources were associated with a local hot-dip galvanizing operation. Motor vehicles were found to be the most significant contributor to fine particle concentrations. Four coarse (sea salt, road dust, 
soil and wood burning) and five fine (wood burning, sulphate, motor vehicles, soil and sea salt) sources were identified in Masterton. Sea salt was found to dominate the coarse fraction all year, while the fine fraction was dominated by wood combustion, particularly during the winter.

A study in Wainuiomata identified five fine (biomass burning, motor vehicles, sulphate, marine aerosol and soil) and three coarse (marine aerosol, road dust and soil) sources (Davy et al. 2012). Biomass burning was found to dominate the fine fraction, particularly during autumn and winter.

Recently, a study in Masterton that focused on identifying $\mathrm{PM}_{10}$ sources and their contributions on an hourly time-scale at two locations within the rural township was reported (Ancelet et al. 2012b). Using PMF, four $\mathrm{PM}_{10}$ source contributors were identified at each location: biomass burning, vehicles, crustal matter and marine aerosol. Biomass burning was found to be the most significant contributor to the evening and morning peak $\mathrm{PM}_{10}$ concentrations measured. This result suggests that peak $\mathrm{PM}_{10}$ concentrations in the morning were the result of people re-lighting their fires. Ancelet et al. (2012b) also found that katabatic drainage was the most likely $\mathrm{PM}_{10}$ transport mechanism, with consistently higher $\mathrm{PM}_{10}$ concentrations measured downwind of the urban area.

\subsubsection{South Island}

Spatial variations in $\mathrm{PM}_{10}$ concentrations were studied between Rangiora (11 000 people) and Kaiapoi (9 000 people) in the Canterbury region (Hamilton et al. 2004). The study found that both towns experience extremely elevated $\mathrm{PM}_{10}$ concentrations, particularly in older residential neighbourhoods. During settled anticyclonic conditions conducive to high $\mathrm{PM}_{10}$ concentrations, it was found that nocturnal drainage flows could carry $\mathrm{PM}_{10}$ plumes from Rangiora to Kaiapoi, causing $\mathrm{PM}_{10}$ concentrations in Kaiapoi to be further increased.

Alexandra (4 800 people) is situated in a sheltered inland basin in central Otago and experiences extremely poor air quality during the winter. A study of the air pollution meteorology in Alexandra found that the best predictor of an air pollution event that night was when air temperatures at 08:00 or 09:00 were less than $0^{\circ} \mathrm{C}$ and wind speeds were less than $0.5 \mathrm{~m} \mathrm{~s}^{-1}$ (Tate and Spronken-Smith 2008). These criteria accounted for approximately $60 \%$ of high pollution days. The authors also concluded 
that variable emission rates, the complexity of local drainage flows and the occurrence of hoar frost limited the more accurate prediction of high pollution episodes. A later study of $\mathrm{PM}_{10}$ meteorology in Alexandra focused on understanding the bi-modal peak observed in $\mathrm{PM}_{10}$ concentrations during the evening (Figure 4.1) (Tate et al. 2011). The authors concluded that variations in the strengths of drainage winds were responsible for shifting plumes away from and then towards the monitoring site, suggesting that changes in the horizontal transport of plumes, and not changes in emissions from domestic heating, was responsible for the bi-modal peak observed during the evening. In addition to Christchurch, Brown et al. (2005) also reported $\mathrm{PAH}$ and $\mathrm{BaP}$ concentrations during the summer and winter in Alexandra and Dunedin (100 000 people). Winter PAH and BaP concentrations were higher than those during the summer, with average winter PAH concentrations of 128.87 and 5.83 ng $\mathrm{m}^{-3}$ in Alexandra and Dunedin, respectively, and average winter $\mathrm{BaP}$ concentrations of 17.96 and $0.76 \mathrm{ng} \mathrm{m}^{-3}$ in Alexandra and Dunedin, respectively. The exceptionally high PAH and BaP concentrations in Alexandra demonstrate that even though it is a very small community, Alexandra has a serious PM pollution problem.

Another study of air pollution meteorology in central Otago found that, on high pollution days, a stable boundary layer forms and is typically present for 18 hours (17:00-11:00) (Broadbent et al. 2010). A strongly stable layer is also formed near the ground surface, with an average depth of $25 \mathrm{~m}$, limiting the dispersion of pollutants emitted inside this layer. Broadbent et al. (2010) also suggested that a $50 \%$ reduction in domestic and industrial emissions was required for adherence to the NESAQ.

A study of particle-phase semivolatile organic contaminants (SOCs) at a remote site in New Zealand's Southern Alps found that numerous pesticides, PAHs and polychlorinated biphenyls had been transported from a number of origins (Lavin et al. 2012). A number of species measured at the site were identified as being Australian in origin, while chlorpyrifos was largely found to originate from the Canterbury Plains.

High $\mathrm{PM}_{10}$ episodes in Invercargill (50 000 people) were characterized by strong temperature inversions, large domestic heating emissions, spatial inhomogeneity in $\mathrm{PM}_{10}$ concentrations and diurnally switching winds transporting $\mathrm{PM}_{10}$ (Conway et al. 2007). The authors found that $\mathrm{PM}_{10}$ concentrations were highest in the southern residential suburbs of the city, moderate in the central business district 
and low in areas outside the city boundaries, suggesting that the Environment Southland monitoring site is not ideally suited for measuring maximum $\mathrm{PM}_{10}$ concentrations.

\subsection{Source-Specific Emissions}

This section describes specific research on PM emission sources known to impact on urban air quality in New Zealand and provides a basis for better understanding the nature of the PM composition from each source and the parameters that affect the chemistry and quantity of emissions. The complex nature of ambient PM composition and the physical and chemical transformations that PM can undergo between emission source and sampling location can make such source specific studies invaluable for air quality management purposes.

\subsubsection{Wood burners}

Since emissions from domestic heating significantly reduce wintertime air quality across New Zealand, it is important to characterize emissions from wood burners. As part of the NESAQ, New Zealand has introduced standards for new wood burners (particulate emissions of $1.5 \mathrm{~g} \mathrm{~kg}^{-1}$ or less, $65 \%$ efficiency). A report on the emission auditing of new wood burners in Australia and New Zealand has been reported (Todd 2008). A comparison study involving a NESAQ-compliant wood burner and a 15-year old wood burner found little difference in PM emissions between the two burners (Ancelet et al. 2010). A wider study by Auckland Council involving the same wood burners did, however, find that total PM emissions from the NESAQ-compliant wood burner were lower than from the older burner (Xie and Mahon 2010). Emissions from the NESAQ-compliant burner were also well above those reported for 'real-life' testing in Tokoroa, Nelson, Rotorua and Tamaranui (Kelly et al. 2007; Smith et al. 2008). These 'real-life' studies suggested that a significant reduction in PM emissions is observed with NESAQ-compliant wood burners. Ancelet et al. (2010) and Xie and Mahon (2010) also reported that wood burner operation played a significant role in PM emissions, with start-up and low burn phases producing the highest concentrations of PM. Ancelet et al. (2010) also evaluated PAH emissions from the NESAQ-compliant wood burner and the older 
wood burner. PAH emissions from the older wood burner were markedly higher than those from the newer burner, particularly during the high burn phase, when the combustion efficiency is expected to be at a maximum.

$\mathrm{PM}$, elemental and PAH emissions from an NESAQ-compliant burner have also been evaluated based on the type of wood used as fuel (Ancelet et al. 2011a). Softwood was found to have the lowest PM emission factors, but highest PAH emission factors. Elemental analysis revealed that approximately $4 \%$ of the PM emitted was composed of inorganic species, with the remaining species being predominantly OC followed by EC. This study highlights the fact that PM concentrations alone do not provide enough essential information about the potential toxicity of particles.

The effect of wood burners on indoor air quality has also been investigated (Longley and Gadd 2011). Using one minute data, the authors found that indoor $\mathrm{PM}_{10}$ in households using wood burners was characterized by short (1-5 minute), elevated $\mathrm{PM}_{10}$ concentrations, followed by a rapid exponential decay in $\mathrm{PM}_{10}$ concentrations. The $\mathrm{PM}_{10}$ elevations coincided with the wood burner start-up and studies in a 'control' home found that peak $\mathrm{PM}_{10}$ concentrations were linked to the lighting process, which is dependent on the operator. Longley and Gadd (2011) also found that no linear relationship existed between ambient and indoor $\mathrm{PM}_{10}$ concentrations and that $\mathrm{PM}_{10}$ exposure in wood burning households can be substantially higher than in non-wood burning homes and ambient air.

\subsubsection{Motor Vehicles}

Tunnel studies are extremely useful for understanding 'real-world' PM emissions from motor vehicles. A study in the Mount Victoria Tunnel in Wellington found that the average $\mathrm{PM}_{2.5}$ concentration during peak hours was $67.6 \mu \mathrm{g} \mathrm{m} \mathrm{m}^{-3}$ (Ancelet et al. 2011b). Carbonaceous species were found to be dominant in the tunnel, contributing 63-73\% to the total PM in the tunnel. Of these carbonaceous species, PAHs were found to contribute $0.1 \%$ to the total $\mathrm{PM}_{2.5}$ and low molecular weight PAHs were enriched, indicative of diesel-fuelled vehicles.

A source apportionment study in the Johnstone's Hill tunnel identified six primary sources contributing to $\mathrm{PM}_{10}$ concentrations: light duty vehicles, heavy commercial vehicles, smoky vehicles, re-suspended road dust, biomass burning and 
marine aerosol (Davy et al. 2011). Vehicle exhaust emissions and road dust were the predominant sources of $\mathrm{PM}_{10}$ in the tunnel, while, on occasion, $\mathrm{PM}_{10}$ sources from outside the tunnel (mainly marine aerosol) had a significant influence on $\mathrm{PM}_{10}$ concentrations inside the tunnel. Of the vehicle emissions, heavy commercial vehicles had the highest overall contribution to $\mathrm{PM}_{10}$ mass.

\subsection{Conclusions}

The focus of this work has been to review what is currently known about PM pollution in New Zealand. It is evident that New Zealand's unique geography and remote oceanic location play important roles in measured PM concentrations. It is also clear from the studies reviewed that, during winter, many urban areas experience poor air quality, largely as a result of domestic emissions from wood combustion coupled with poor meteorological dispersion conditions. Based on the studies presented in this work, a number of new objectives relating to PM have been identified and will be briefly discussed.

Since PM pollution during the winter is largely the result of domestic wood combustion, a $\mathrm{PM}_{10}$ standard alone is unlikely to be providing an adequate level of protection for the exposed populace due to the fact that combustion derived PM is primarily $\mathrm{PM}_{2.5}$ (and mostly much smaller, $<\mathrm{PM}_{1}$ ). It is likely that during pollution events $\mathrm{PM}_{10}$ is largely composed of $\mathrm{PM}_{2.5}$. As such, including a $\mathrm{PM}_{2.5}$ standard should better reflect both our increased understanding of the PM pollution problem in New Zealand and measures taken overseas. The World Health Organization suggests a $\mathrm{PM}_{2.5}$ standard of $25 \mu \mathrm{g} \mathrm{m}{ }^{-3}$ and the United States has adopted a $\mathrm{PM}_{2.5}$ standard of 35 $\mu g \mathrm{~m}^{-3}$ (both 24-hour averages). At the least, more $\mathrm{PM}_{2.5}$ monitoring needs to be undertaken in New Zealand to understand the extent of the $\mathrm{PM}_{2.5}$ problem and to better inform policy makers.

Many pollutants covered by AAQG are not routinely monitored throughout New Zealand. It is clear from the studies discussed in this work that concentrations of hazardous species associated with PM can be divided into two categories: inorganic (e.g. arsenic) and organic compounds (e.g. BaP). Inorganic species associated with $\mathrm{PM}$ in ambient air usually arise from some industrial process emission, or more likely in the New Zealand context, from the combustion of fuels that contain such species as constituents (arsenic from the use of treated timber as fuel). Monitoring can then be 
targeted towards those species known to be associated with fuels (or potential fuels) used in combustion processes. On the other hand most organic pollutants associated with PM result from incomplete combustion and are therefore generated in situ. Covering the swathe of such compounds produced is more difficult due to sampling requirements and not least, cost considerations. Targeting those that are known to be present in higher concentrations and/or more toxic is probably the most effective from a cost-benefit perspective. Certainly PAHs must be high on the list, and for PM those PAHs with four rings and above.

As these species are not routinely monitored, determining whether their concentrations are exceeding their respective AAQGs (as annual averages) is difficult. Given their detrimental health effects, it is important to understand whether pollutants with AAQG values are a problem in New Zealand.

Incorporating the two points already mentioned, long-term monitoring campaigns focusing on these species and others (elements, OC, etc.) should be undertaken to better understand temporal variations in species concentrations and source contributions. Long-term studies would not only allow for a more accurate assessment of the yearly concentrations of species like $\mathrm{BaP}$ and arsenic, but would also provide excellent datasets for source apportionment studies and a better understanding for managing air quality in different regions. The power of long-term air pollution studies for assessing the impact of mitigation strategies and air quality policy should not be underestimated.

Location-specific sources are also likely to have a significant impact on local air quality in New Zealand. Targeted ambient monitoring for industrial point sources, shipping emissions and vehicular sources (not just tailpipe emissions, but also the abrasion of mechanical parts such as brakes for $\mathrm{Cu}, \mathrm{Zn}, \mathrm{Sb}$ and $\mathrm{Ba}$ ) would allow for a better understanding of the contributions of these sources to ambient $\mathrm{PM}_{10}$ and $\mathrm{PM}_{2.5}$ concentrations and the exposure risk for local populations.

Another point worth mentioning is that, while this work focused largely on research published in peer-reviewed journals, there has been a significant amount of local research reported only for end-users. These reports make up the 'grey' literature and it is encouraged that as much research as possible be reported to the wider air quality community. This journal provides an excellent means of reporting valuable information and publication in it or other peer-reviewed media is greatly encouraged. 


\subsection{Acknowledgements}

The authors thank Tamsin Mitchell for providing manuscripts of older studies that could not be obtained. This work was partially funded by the New Zealand Ministry of Science and Innovation under contract C05X0903. 


\section{Research objectives}

The aim of this research was to investigate the chemical composition of PM to identify the primary sources of PM emissions in New Zealand urban environments. Particulate matter emission patterns and transport within urban environments were also investigated to gain an understanding of the effect of meteorological and topographical conditions on air pollution episodes. The study by Trompetter et al. (2010), discussed in Chapter 4.4, identified common diurnal patterns in $\mathrm{PM}_{10}$ concentrations during the winter throughout New Zealand. Critically, the two peaks in $\mathrm{PM}_{10}$ concentrations apparent throughout New Zealand can cause exceedances of the NES. While it is evident from Chapter 4 that residential wood combustion for home heating is the most significant source of $\mathrm{PM}_{10}$ in many locations during the winter, the sources and/or factors responsible for the morning peak in $\mathrm{PM}_{10}$ concentrations were not clear. Furthermore, nothing was known about how source contributions vary throughout a day. As such, this research targeted these knowledge gaps to answer fundamental questions about PM composition, emission sources and atmospheric processing, information that is critical to understanding and managing air pollution in New Zealand. This work has, for the first time, investigated PM source variations at an hourly resolution at multiple sites within an airshed to identify the sources responsible for the observed diurnal patterns in $\mathrm{PM}_{10}$ concentrations (Figure 4.1) and has also investigated PM transport (horizontally and vertically) within an airshed.

\subsection{Thesis overview}

When undertaking a source apportionment study, it is important to gain an understanding of particle emissions from an emission source. As such, Chapters 6 and 7 present the results of separate studies on wood burners commonly used throughout New Zealand. Chapter 6 presents a comparison of PM and PAH emissions from a modern, NES-certified wood burner and an older, non-compliant wood burner. Chapter 7 presents an evaluation of PM, PAH and elemental emissions from a separate, NES-compliant wood burner. To evaluate PM emissions from motor vehicles, PM samples were collected from the Mount Victoria Tunnel in Wellington, New Zealand. Chapter 8 focuses on carbonaceous emissions from motor vehicles. 
Chapters 9, 10 and 11 present the results of intensive PM sampling campaigns in Masterton, Nelson and Alexandra, respectively. Figure 5.1 presents the locations of Masterton, Alexandra and Nelson.

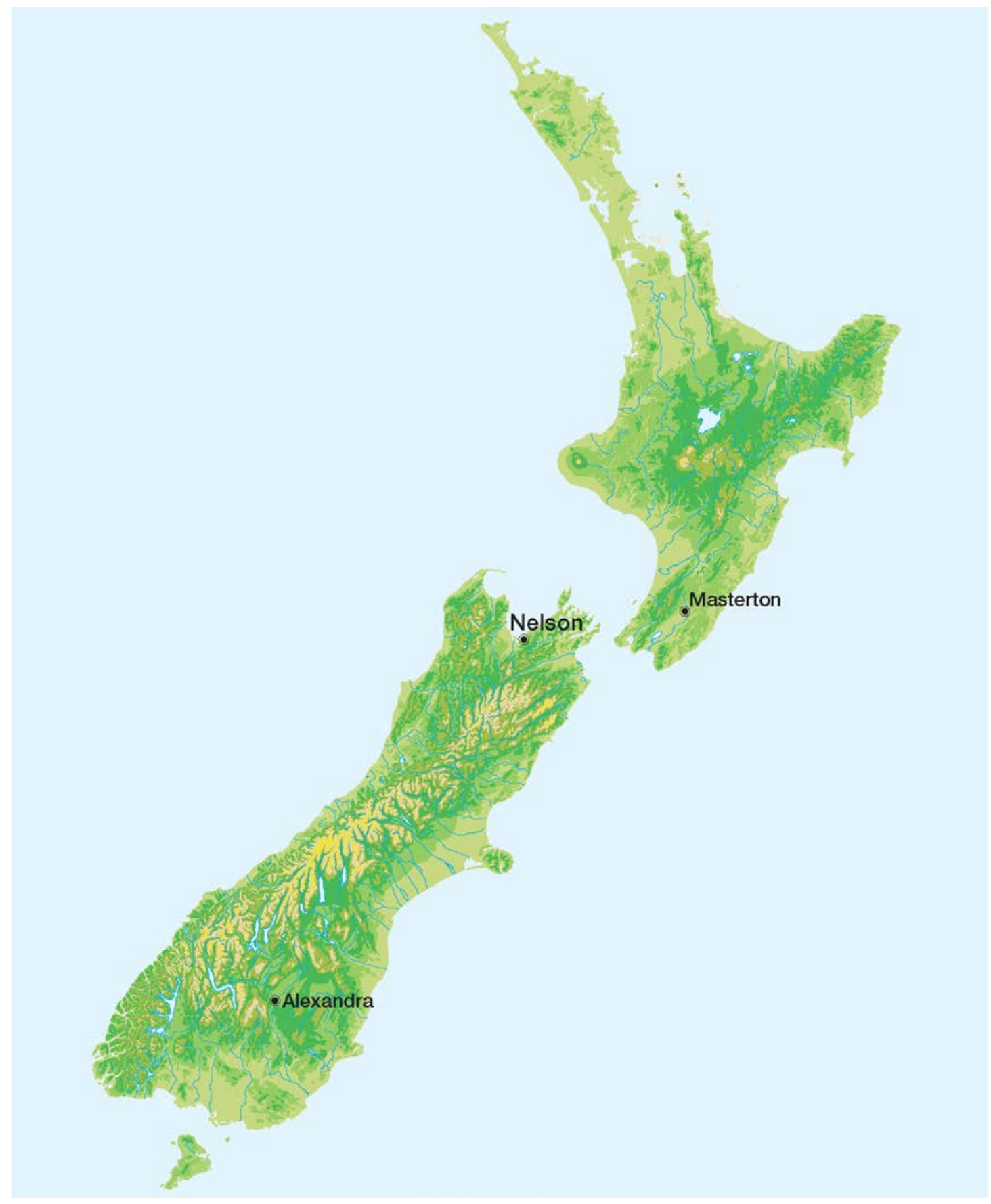

Figure 5.1 Locations of Masterton, Nelson and Alexandra. 


\section{A comparison of particulate and particle-phase PAH emissions from a modern wood burner with those of an old wood burner}

\subsection{Synopsis}

This chapter has been reproduced in full, with permission, as it appears in Air Quality and Climate Change, 2010. Ancelet, T., Davy, P. K., Trompetter, W. J., Markwitz, A. and Weatherburn, D. C., 2010. A comparison of particulate and particle-phase PAH emissions from a modern wood burner with those of an old wood burner. Air Quality and Climate Change, 44, 21-24. *Note*: The National Environmental Standards (NES) have been updated since this manuscript was published. A full description of the revised NES was provided in Chapter 4.

In an effort to establish reliable emission factors and assess wood burner improvements, particle-phase PAH emissions were determined for the combustion of pine under different operating conditions from a modern, NES-compliant wood burner and an older non-compliant burner. Particle-phase PAH concentrations were determined for 84 different test cycles by thermal desorption GC/MS. The analysis revealed that total particulate matter emissions were similar from both burners for our data set, with emissions from the NES-compliant burner above the prescribed level. Total particulate emissions for both burners were determined to be highest during low burn, with start-up also producing significant particulate emissions. PAH emissions were found to vary significantly between the two burners, with emissions from the old burner considerably higher than those from the NES-compliant burner. Operating condition had little effect on PAH emissions from the old burner, but played an important role in emissions from the NES-compliant burner, with start-up producing the highest PAH emissions. The results presented in this study improve the current knowledge base for pollutants emitted from wood combustion and their emission factors in New Zealand. 


\subsection{Introduction}

The utilisation of wood as a renewable energy source has been gaining interest throughout many parts of the world as a way to reduce emissions of greenhouse gases. In New Zealand, 41 \% of households use wood as a source of heating, demonstrating the relative importance of wood burners for home heating throughout the country (Statistics New Zealand 2006). While wood is appealing as a renewable fuel, it has been shown to significantly contribute to local air pollution episodes throughout New Zealand and Australia (Keywood et al. 2000; McGowan et al. 2002; Davy et al. 2005; Trompetter et al. 2010).

Particulate Matter (PM) has been well documented to have adverse effects on human health (Schwartz 1991; Nel 2005; Russell and Brunekreef 2009). The health risk posed by PM is due to a number of factors, namely, particle size, surface area and chemical composition (Hannigan et al. 2005; Staniswalis et al. 2005). Residential combustion of wood results in the emission of a complex mixture of varying PM sizes and composition including elemental carbon, inorganic components (fly ash), volatile organic compounds (VOCs), other semi- or non-volatile organic components and polycyclic aromatic hydrocarbons (PAHs) (Lighty et al. 2000). Emissions of PAHs are of particular concern since many are known or suspected to be carcinogens and mutagens (IARC 1998; Rybicki et al. 2006). Benzo[a]pyrene is classified as a priority pollutant by the Ministry for the Environment in New Zealand and has an annual average guideline concentration of $3 \mathrm{ng} \mathrm{m}^{-3}$ (Ministry for the Environment 2002a). Ambient monitoring in Auckland and Christchurch has revealed that concentrations of benzo[a]pyrene can be highly elevated during winter months, when wood burning for residential heating is common (Cavanagh et al. 2009).

In October 2004, the New Zealand government introduced National Environmental Standards (NES) for air quality. The fourteen standards include: seven standards banning activities that discharge significant quantities of dioxins and other toxins into the air; five standards for ambient (outdoor) air quality; a design standard for new wood burners installed in urban areas (particulate emissions of $1.5 \mathrm{~g} / \mathrm{kg}$ or less, $65 \%$ efficiency); and a requirement for all landfills with over 1 million tonnes of refuse to collect greenhouse gas emissions. The NES prescribes a mean daily maximum concentration of $50 \mu \mathrm{g} \mathrm{m}{ }^{-3}$ for $\mathrm{PM}_{10}$, which is not to be exceeded more 
than once per year by 2013. Regions which breach the NES after 2013 will be forced to decline all ‘discharge-to-air’ permits for emissions containing PM10.

Emission inventories can be used to estimate emissions from sources in order to establish relative contributions to air pollution and project future scenarios based on a range of interventions for air quality management purposes. As an alternative to emission inventories, receptor modeling can be used to determine the sources contributing to local PM concentrations by measuring elemental and chemical components of the PM. Prior knowledge of elemental and chemical composition is required to identify sources and apportion mass contributions accurately.

In order to assess the improvement in wood burner technology after the introduction of the NES design criteria for wood burners and to establish PAH emissions factors, this study compared total PM emissions and particle-phase PAH emissions from a NES-compliant Masport LE3000 burner (AS/NZS standard emission factor $=0.76 \mathrm{~g} / \mathrm{kg}$ ) with the emissions from a 15-year old Masport Belvedere burner. The effect of burner operating condition on PAH emissions was also investigated.

\subsection{Experimental}

\subsubsection{Wood burner emissions sampling}

The particulate matter emissions from Masport LE3000 and Masport Belvedere wood burners were measured by Spectrum Laboratories Limited on behalf of Auckland Regional Council. PM emissions from 60 different test cycles for the Masport Belvedere and 24 different test cycles for the Masport LE3000 were used in this study. The burners were operated in a laboratory using a calorimeter room and sampling train prescribed in standard AS/NZS 4012/3. In order to simulate real-life operations, each test burning cycle consisted of three operating conditions from cold start to high burn and low burn. High burn and low burn were achieved by setting the heat output control to achieve a maximum or minimum burning rate respectively. The start-up phase lasted for approximately 50 minutes with the high burn and low burn phases lasting approximately 120 minutes each. PM emissions were collected throughout the whole operating cycle and were determined for each operating 
condition. Loaded filters were kept refrigerated at $4^{\circ} \mathrm{C}$ until just prior to $\mathrm{PAH}$ analysis.

Locally sourced commercial pine was used as fuel and included pieces with some bark and/or knots which may be a source of variation between individual tests.

\subsubsection{PAH analysis}

The concentrations of sixteen PAHs classified as priority pollutants by the United States Environmental Protection Agency (USEPA) were determined using thermal desorption GC/MS (TD/GC/MS). The analysis of PAHs in particulate matter using thermal desorption has previously been shown to provide comparable results to USEPA method TO-13A in the analysis of NIST reference materials (Waterman et al. 2000; Falkovich and Rudich 2001; Bates et al. 2008).

Prior to analysis, a small sample was taken from each filter using a punch (6.35 mm diameter). Analysis of multiple punches from the same filter revealed that each punch was highly representative of the deposited PAHs on the whole filter. A total of 84 sets (front and back) of glass fibre filters were analyzed. The filter punches were then placed into individual, empty stainless steel cups (Frontier Lab) and spiked with naphthalene- $d_{8}$, phenanthrene- $d_{10}$ and pyrene- $d_{12}$ obtained from Aldrich. PAHs were not detected in the empty cups or on blank filters. Once spiked, the samples were placed in a Frontier Lab Auto-Sampler in preparation for desorption. Thermal desorption was performed using a Frontier Lab Double-Shot Pyrolyzer in the single shot mode at $300{ }^{\circ} \mathrm{C}$ for 3 minutes in order to avoid organic compound degradation. The desorbed analytes were trapped on a cryogenically cooled Frontier Lab Micro-Jet Cryo Trap until desorption was complete. The completeness of desorption was confirmed to be greater than $99 \%$ by a repeated desorption of an already analyzed filter. The pyrolyzer was directly interfaced to a GC/MS unit (Agilent 7890A GC and 5975C MSD) with helium as the carrier gas.

Upon completion of the desorption step, the cooled analytes were released to an Agilent HP-5 column (30 m x $320 \mu \mathrm{m}$ x $0.25 \mu \mathrm{m}$ ). The GC oven was held at $80^{\circ} \mathrm{C}$ for $3 \mathrm{~min}$, ramped at $6^{\circ} \mathrm{C} / \mathrm{min}$ to $150^{\circ} \mathrm{C}$, at $3^{\circ} \mathrm{C} / \mathrm{min}$ to $260^{\circ} \mathrm{C}$ and at $6^{\circ} \mathrm{C} / \mathrm{min}$ to 280 ${ }^{\circ} \mathrm{C}$ when it was held constant for $12 \mathrm{~min}$. The MS detector was operated in Selected Ion Monitoring (SIM) mode. PAHs were quantified using relative response ratios determined from certified authentic and isotopically labeled internal standards. A 
standard PAH solution used for calibration was obtained from Supelco and was used as received (TCL Polynuclear Aromatic Hydrocarbons Mix). Adequate linearity $\left(r^{2} \geq\right.$ 0.976) was obtained from calibrations for all sixteen PAHs.

\subsection{Results}

\subsubsection{Total particulate emissions}

A detailed analysis of particulate matter emissions from the two wood burners used in this study, along with results from another burner for the combustion of pine, macrocarpa and blue gum has been reported previously (Xie and Mahon 2010). Total particulate emissions from both wood burners tested are presented in Table 6.1. It is evident from Table 6.1 that the total particulate emissions from both wood burners are highest during low burn, probably due to poor combustion conditions resulting from the lower temperature and decreased combustion efficiency.

Table 6.1 Particulate emission factors for Masport Belvedere $(n=60)$ and Masport LE3000 $(n=24)$

\begin{tabular}{|c|c|c|c|c|c|c|}
\hline \multirow[b]{2}{*}{ Phase } & \multicolumn{3}{|c|}{$\begin{array}{l}\text { Masport Belvedere PM emission factors } \\
(\mathrm{g} / \mathrm{kg})\end{array}$} & \multicolumn{3}{|c|}{$\begin{array}{l}\text { Masport LE3000 PM emission factors } \\
(\mathrm{g} / \mathrm{kg})\end{array}$} \\
\hline & Start-Up & High Burn & Low Burn & Start-Up & High Burn & Low Burn \\
\hline Average & 7 & 8 & 17 & 7 & 4 & 16 \\
\hline Median & 5 & 7 & 19 & 6 & 4 & 14 \\
\hline Std Dev & 4 & 6 & 12 & 5 & 3 & 12 \\
\hline
\end{tabular}

Emissions during start-up, while apparently similar to those during high burn, could also be considered high due to the shorter duration of the start-up cycle. Also evident from Table 6.1 is that particulate emissions from both wood burners are very similar under start-up and low burn conditions with average values of $7 \pm 4 \mathrm{~g} / \mathrm{kg}$ and 7 $\pm 5 \mathrm{~g} / \mathrm{kg}$ during start-up and $17 \pm 12 \mathrm{~g} / \mathrm{kg}$ and $16 \pm 12 \mathrm{~g} / \mathrm{kg}$ during low burn for the Masport Belvedere and Masport LE3000, respectively. Only during high burn were particulate emissions slightly different, with emissions from the NES compliant Masport LE3000 averaging $4 \pm 3 \mathrm{~g} / \mathrm{kg}$, well above the NES prescribed $1.5 \mathrm{~g} / \mathrm{kg}$. The wider study by Auckland Regional Council did find that total PM emissions from the Masport LE3000 were lower than PM emissions from the Masport Belvedere (Xie and Mahon 2010). The PM emissions from the Masport LE3000 are well above the 
PM emissions for "real-life" testing in Tokoroa, Nelson, Rotorua and Tamaranui (Kelly et al. 2007; Smith et al. 2008). These studies suggest that a significant reduction in particulate matter is observed with NES-compliant wood burners, over older, non-compliant burners. Despite emissions from our data set being greater than the NES, these results are in good agreement with previously reported softwood emission factors of 2.9-9.0 g/kg (McDonald et al. 2000), $13.0 \pm 4 \mathrm{~g} / \mathrm{kg}$ (Hildemann et al. 1991) and $15.8 \mathrm{~g} / \mathrm{kg}$ (Gras et al. 2002).

\subsubsection{PAH emissions}

Despite total particulate emissions being similar between the two wood burners, PAH emissions from the burners were found to be significantly different. Table 6.2 presents PAH emission factors from both burners studied. The Masport Belvedere produced higher quantities of every $\mathrm{PAH}$ analysed, with total PAH emissions almost 13 times greater than those from the Masport LE3000.

Table 6.2 PAH emission factors for Masport Belvedere $(n=60)$ and Masport LE3000 ( $\mathrm{n}=24)$

\begin{tabular}{|c|c|c|c|c|c|c|}
\hline \multirow[b]{3}{*}{ naphthalene } & \multicolumn{3}{|c|}{ Masport Belvedere (mg/kg) } & \multicolumn{3}{|c|}{ Masport LE3000 (mg/kg) } \\
\hline & Average & Median & Std dev & Average & Median & Std dev \\
\hline & 0.7 & 0.2 & 1 & 0.1 & 0 & 0.2 \\
\hline acenaphthylene & 16 & 3 & 28 & 1 & 0.1 & 2 \\
\hline acenaphthene & 2 & 0.6 & 3 & 0.1 & 0 & 0.2 \\
\hline fluorene & 13 & 3 & 19 & 0.8 & 0.1 & 2 \\
\hline phenanthrene & 201 & 98 & 292 & 10 & 4 & 14 \\
\hline anthracene & 47 & 25 & 63 & 2 & 0.5 & 3 \\
\hline fluoranthene & 114 & 60 & 164 & 9 & 7 & 12 \\
\hline pyrene & 93 & 45 & 137 & 7 & 4 & 10 \\
\hline benzo[a]anthracene & 36 & 21 & 49 & 6 & 3 & 10 \\
\hline chrysene & 45 & 23 & 66 & 6 & 3 & 9 \\
\hline benzo[b]fluoranthene & 0.7 & 0.4 & 0.8 & 0.5 & 0.2 & 0.6 \\
\hline benzo[k]fluoranthene & 0.2 & 0.1 & 0.3 & 0.1 & 0 & 0.1 \\
\hline benzo[a]pyrene & 2 & 0.6 & 3 & 0.7 & 0.2 & 1 \\
\hline indeno[1,2,3-c,d]pyrene & 3 & 1 & 4 & 1 & 0.3 & 2 \\
\hline dibenzo[a,h]anthracene & 0.4 & 0.1 & 0.5 & 0.3 & 0.1 & 0.6 \\
\hline benzo[g,h,i]perylene & 2 & 0.5 & 3 & 0.5 & 0.2 & 0.7 \\
\hline Sum & 576 & 281.5 & & 45 & 23 & \\
\hline
\end{tabular}


The dramatic difference in PAH emissions is likely to be due to the more modern design of the Masport LE3000 creating more efficient combustion conditions than the Masport Belvedere, resulting in fewer PAH emissions. The significant difference in $\mathrm{PAH}$ emissions is interesting with regards to ambient air monitoring, since it is obvious that PM emissions alone are not a sufficient indicator of $\mathrm{PAH}$ emissions and future air quality regulations may need to address this. The predominant $\mathrm{PAH}$ emissions were phenanthrene, fluoranthene, pyrene, benzo[a]anthracene and chrysene for both burners.

In order to compare PAH emissions between the two wood burners in more detail, PAH emissions during start-up, high burn and low burn were investigated and compared between the two burners. Figure 6.1 presents a comparison of PAH emissions from both wood burners during start-up. It is obvious from Figure 6.1 that PAH emissions are significantly higher from the old Masport Belvedere burner than from the Masport LE3000. It can also be seen that relative PAH emissions are different between the two burners. PAH emissions from the Masport Belvedere are dominated by phenanthrene; however, this is not the case for the Masport LE3000 where fluoranthene and pyrene emission factors were higher than phenanthrene. 


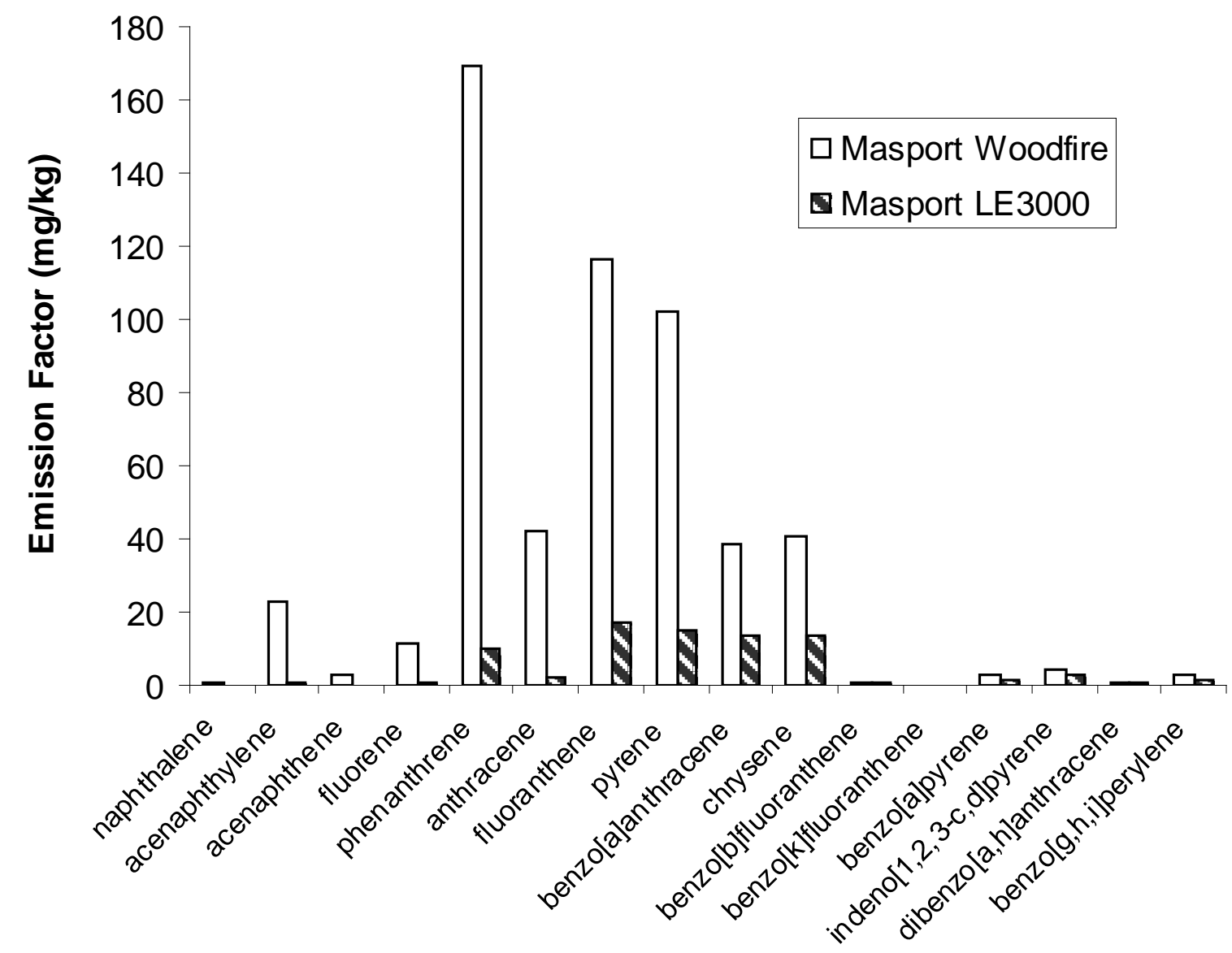

Figure 6.1 Comparison of PAH emissions during start-up between Masport Belvedere $(\mathrm{n}=20)$ and Masport LE3000 (n=8) 


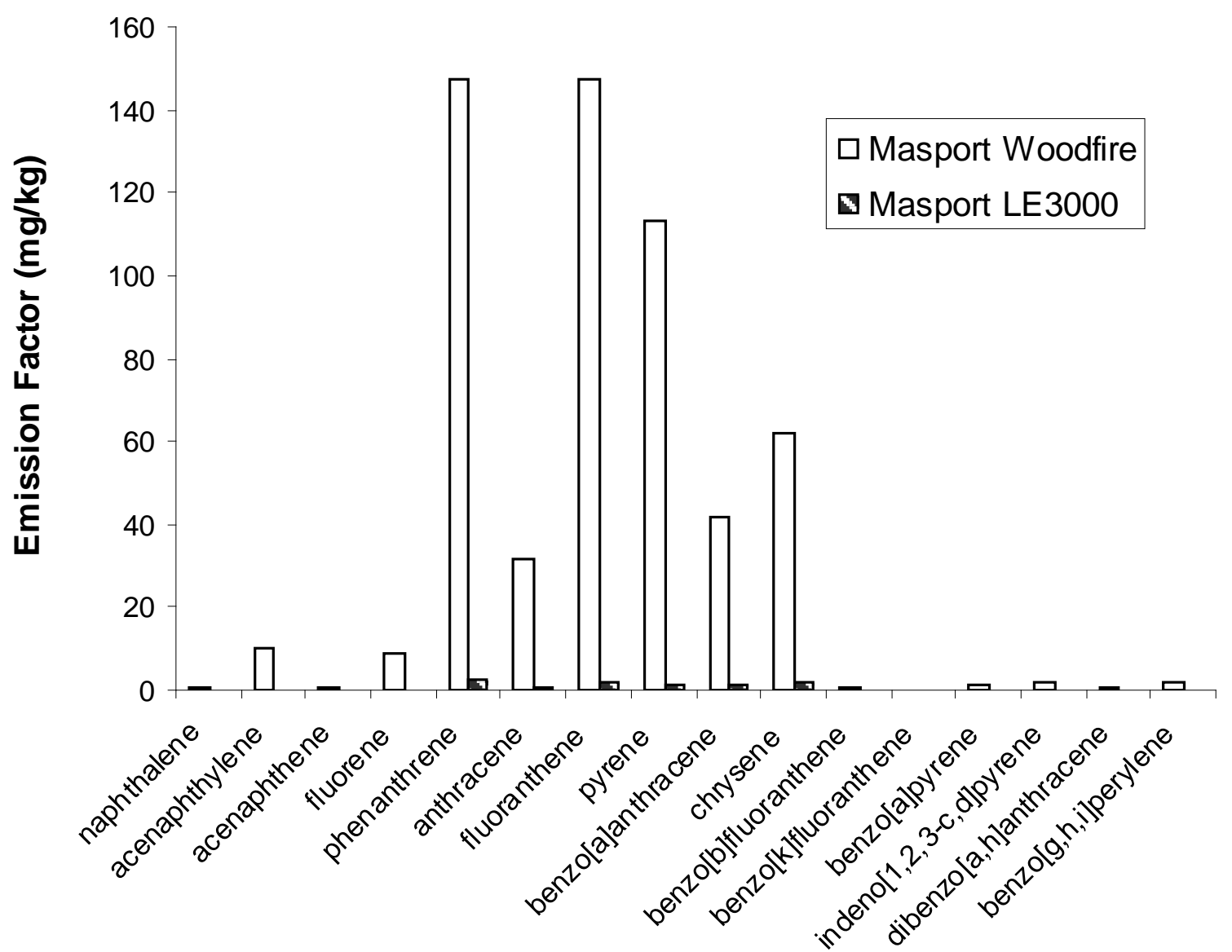

Figure 6.2 Comparison of PAH emissions during high burn between Masport Belvedere $(\mathrm{n}=20)$ and Masport LE3000 (n=8) 


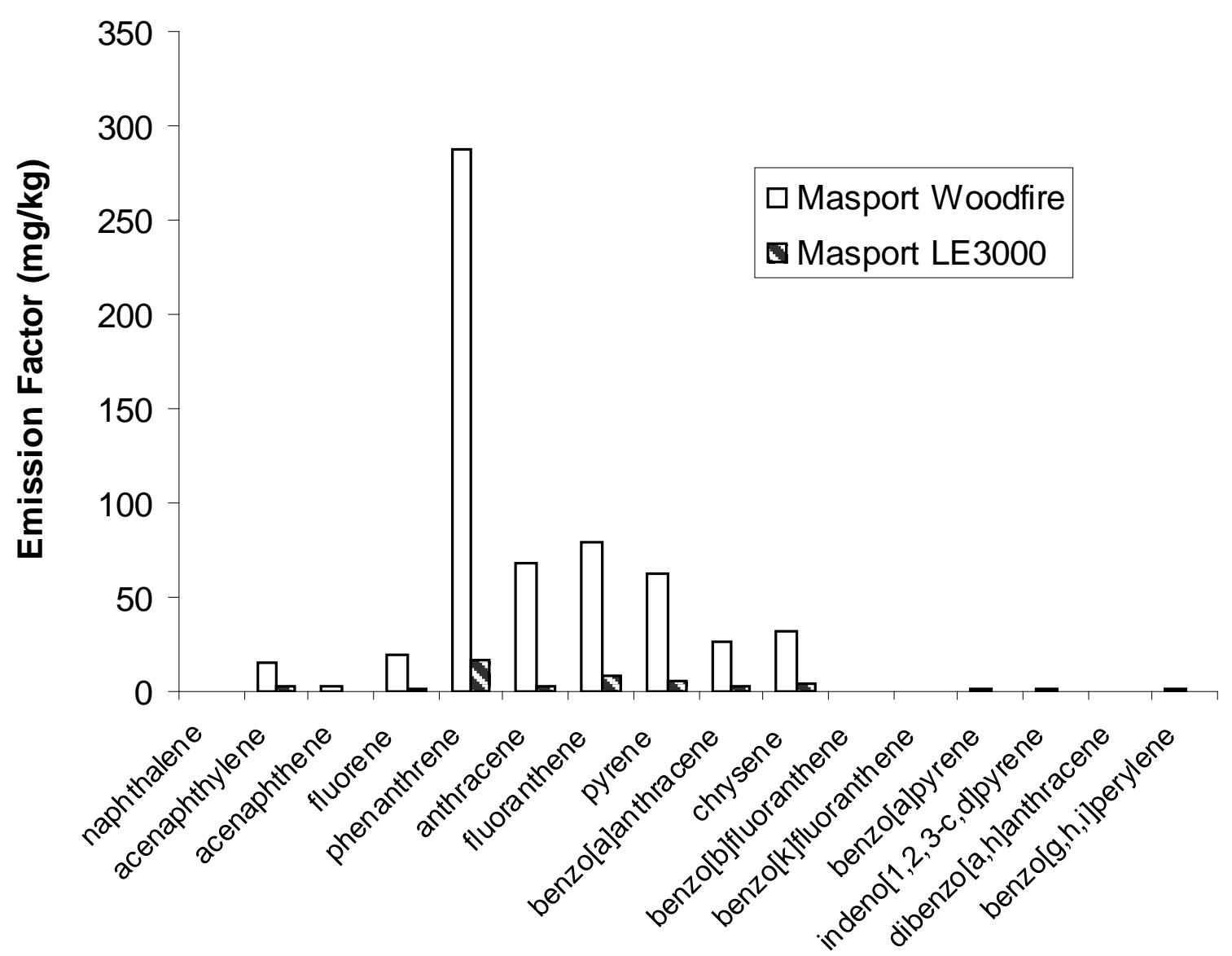

Figure 6.3 Comparison of PAH emissions during low burn between Masport Belvedere $(\mathrm{n}=20)$ and Masport LE3000 (n=8)

Figure 6.2 presents a comparison of $\mathrm{PAH}$ emissions from both burners during high burn. PAH emissions during high burn would be expected to be significantly lower than emissions during start-up and low burn, since combustion is likely to be most efficient under high burn conditions due to a greater air supply. Figure 6.2 shows that PAH emissions from the Masport LE3000 are markedly lower than emissions during start-up (Figure 6.1). There is, however, insignificant change in PAH emissions from the Masport Belvedere during high burn compared to start-up. This is probably a reflection of the older wood burner design resulting in more unfavorable combustion conditions within the burner.

PAH emissions during low burn for the two burners are presented in Figure 6.3. Again, emissions from the Masport LE3000 burner are significantly lower than PAH emissions from the Masport Belvedere. Interestingly, emissions of phenanthrene were determined to be at their maximum during low burn, with emissions of the other 15 PAHs below their corresponding emission levels during start-up. 


\subsection{Conclusions}

This study assessed the improvement in wood burner technology after the introduction of NES wood burner design standards by comparing total particulate matter emissions and particle-phase $\mathrm{PAH}$ emissions under different operating conditions from one brand of NES-compliant burner and a 15-year old burner of the same brand. From the studied dataset, total particulate matter emissions showed little difference between the two burners, with a minor difference in emissions coming during the high burn cycle, where combustion conditions are likely be most efficient. While total particulate matter emissions were similar between the two burners, it was found that $\mathrm{PAH}$ emissions were significantly higher from the older Masport Belvedere burner. Average total PAH emission factors were determined to be 576 $\mathrm{mg} / \mathrm{kg}$ and $45 \mathrm{mg} / \mathrm{kg}$ for the Masport Belvedere and Masport LE3000 respectively. PAH emissions from the Masport Belvedere burner were found to vary little, independent of the operating condition, whereas PAH emissions from the Masport LE3000 burner were found to be significantly lower during high burn when combustion is most efficient. These results suggest that the current approach of basing wood burner certification on PM emissions alone is inadequate and may need to be updated to include other analyses like PAH emissions.

On the basis of the results presented here the Masport LE3000 burner provided a significant improvement in PAH emissions over the 15-year old Masport Belvedere. This is a significant result that warrants further investigation with a wider class of NES-compliant and non-compliant burners to assess if a similar reduction in PAHs are a general trend. If so, this result would have significant implications for the reduction of air toxics in New Zealand and Australia due to the widespread use of wood burners for residential heating.

\subsection{Acknowledgements}

The authors thank Auckland Regional Council for providing the wood burner emissions samples used in this study. T.A. thanks Andy Phillips for his help with the GC/MS measurements. The work was funded by the New Zealand Foundation for Research, Science and Technology under contract C05X0903. 


\section{Characterisation of particulate matter emissions from a modern wood burner under varying burner conditions}

\subsection{Synopsis}

This chapter has been reproduced in full with permission as it appears in Air Quality and Climate Change, 2011. Ancelet, T., Davy, P. K., Trompetter, W. J., Markwitz, A. and Weatherburn, D. C., 2011. Characterisation of particulate matter emissions from a modern wood burner under varying burner conditions. Air Quality and Climate Change, 45, 21-27.

In an effort to assess PM, particle-phase PAH and elemental emissions from a NES-compliant wood burner, PM samples were collected from the combustion of different wood types, sizes and moisture contents under different burner operating conditions. The woods were chosen based on their commercial availability and the frequency of their use in New Zealand households. The different burner operating conditions used were meant to reflect real-life operating conditions. It was determined that PM emissions were highly dependent on wood type, with emissions from the combustion of hardwood highly elevated compared to emissions from softwood. Despite the higher PM emissions, PAH emissions were found to be highest from the combustion of softwood, indicating that overall PM emissions may not be the best indicator of potential negative health effects. Overall, wood moisture content and wood size were found to play little role in PM emissions, while wood type and burner operating condition had the most significant influence on overall emissions.

\subsection{Introduction}

The utilisation of wood as a renewable energy source has been gaining interest throughout many parts of the world as a way to reduce emissions of greenhouse gases. In New Zealand, $41 \%$ of households use wood as a source of heating, demonstrating the relative importance of wood burners for home heating throughout the country (StatisticsNewZealand 2006). While wood is appealing as a renewable fuel, it has been shown to significantly contribute to local air pollution episodes throughout New 
Zealand and in other parts of the world (Fine et al. 2002; McGowan et al. 2002; Davy et al. 2005; Trompetter et al. 2010; Ward and Lange 2010).

Particulate matter (PM) has been well documented to have adverse effects on human health (Nel 2005; Russell and Brunekreef 2009). The health risk posed by PM is due to a number of factors, namely, particle size, surface area and chemical composition (Hannigan et al. 2005; Staniswalis et al. 2005). Residential combustion of wood results in the emission of a complex mixture of varying PM sizes and composition including elemental carbon, inorganic components (fly ash), volatile organic compounds (VOCs), polycyclic aromatic hydrocarbons (PAHs) and other semi- or non-volatile organic components (Lighty et al. 2000). Emission of PAHs is of particular concern since many are known or suspected to be carcinogens and mutagens (IARC 1998; Rybicki et al. 2006). Benzo[a]pyrene, for example, is classified as a priority pollutant by the Ministry for the Environment in New Zealand and ambient air quality monitoring in Auckland and Christchurch has revealed that concentrations of benzo[a]pyrene can be highly elevated during winter months, when wood burning for residential heating is common (Cavanagh et al. 2009). In many countries residential heating is the most significant source of PAHs in the atmosphere, which makes emission data from wood burners critical for developing more precise emission inventories (Celis et al. 2003; Lee et al. 2005; Junninen et al. 2009).

Significant variability in PM, organic and ionic species emission factors have been previously reported in New Zealand (Scott 2005; Wang et al. 2005a; Wang et al. 2006). This study characterised PM emissions to the atmosphere from a National Environmental Standards (NES)-compliant wood burner typical of those that would be used for home heating in New Zealand. The NES design standard for wood burners installed in urban areas prescribes that the PM emission factor must be $1.5 \mathrm{~g} / \mathrm{kg}$ or less and the wood burner must have a minimum efficiency of $65 \%$. A detailed analysis of particulate matter emissions from the wood burner used in this study, along with the results from two other burners for the combustion of pine has previously been reported (Xie and Mahon 2010). This study aimed to improve the current understanding of pollutants emitted from wood combustion and their emission factors. Parameters studied included the emission of particle-phase PAHs and inorganic elements during different operating conditions (start-up, high burn and low burn). The effects of fuel type, moisture content and whether the wood was split or unsplit on the 
concentration of inorganic elements, particle concentration and PAH concentration were also investigated.

\subsection{Experimental}

\subsubsection{Wood burner emissions sampling}

The particulate matter emissions from a Pioneer Metro Eco wood burner (PM emission factor $<1.0 \mathrm{~g} / \mathrm{kg}$ ) were measured by Spectrum Laboratories Limited on behalf of Auckland Regional Council between December 2007 and March 2009. The burner was operated in a laboratory using a calorimeter room and sampling train prescribed in standard AS/NZS 4012/3. In order to simulate real-life operations, each test burning cycle consisted of three operating conditions from cold start to high burn and low burn on a daily basis, with the filters collected under each operating condition making up a set for the particular wood type combusted. High burn and low burn were achieved by setting the heat output control to achieve a maximum or minimum burning rate respectively. The start-up phase lasted for approximately 50 minutes with the high burn and low burn phases lasting approximately 120 minutes each. PM emissions were collected throughout the whole operating cycle and were determined for each operating condition. Loaded filters were kept refrigerated until just prior to analysis.

Locally sourced commercial wood (pine, macrocarpa and blue gum) was used as fuel and included pieces with some bark and/or knots which may be a source of variation between individual tests. These wood species are the most commonly burned wood types in New Zealand solid fuel appliances for home heating (Scott 2005). The testing was designed to test two levels of moisture content (25\% and 35 \%) as representative of damp and wet wood respectively. The firewood was preprocessed to achieve the required moisture levels. The wood moisture content was measured using a Carrel \& Carrel electrical resistance moisture meter. Another fuel parameter investigated was whether or not the wood was cut (split) or uncut (unsplit) had any effect on the PM emissions. 


\subsubsection{PAH analysis}

The concentrations of sixteen PAHs classified as priority pollutants by the United States Environmental Protection Agency (USEPA) were determined using thermal desorption GC/MS (TD/GC/MS) on 48 quartz fiber filters (four sets each of pine, macrocarpa, split blue gum and unsplit blue gum). The analysis of PAHs in particulate matter using thermal desorption has previously been shown to provide comparable results to USEPA method TO-13A in the analysis of NIST reference materials (Waterman et al. 2000; Falkovich and Rudich 2001; Bates et al. 2008). The analysis of particle-phase PAHs using TD/GC/MS has been previously reported (Ancelet et al. 2010).

\subsubsection{Elemental analysis}

A total of 33 samples (two sets of macrocarpa, four sets of split blue gum and five sets of unsplit blue gum) were collected on $42 \mathrm{~mm}$ diameter Teflon filters and the mass concentrations of airborne particle samples were determined gravimetrically. Elemental concentrations of particle samples were determined by accelerator-based Ion Beam Analysis (IBA) and black carbon (BC) was determined by optical reflectance at the New Zealand Ion Beam Facility (Trompetter et al. 2005). The full suite of analyses included Proton-induced X-ray Emission (PIXE); Proton-induced Gamma-ray Emission (PIGE); Rutherford Backscattering (RBS) and Proton Elastic Scattering Analysis (PESA). Black carbon was measured using the M43D Digital Smoke Stain Reflectometer.

\subsubsection{Total carbon}

Total carbon concentrations for 12 samples (one set each of pine, macrocarpa, split blue gum and unsplit blue gum) of quartz fiber filters were determined using elemental analysis - isotope ratio mass spectrometry (EA/IRMS). The isotope analysis was performed by the Stable Isotope Laboratory at the National Isotope Centre in Gracefield, New Zealand using a dual inlet GVI IsoPrime mass spectrometer with leucine as a working standard. 


\subsubsection{Scanning electron microscopy (SEM)}

Scanning electron microscopy (SEM) and energy dispersive spectroscopy (EDS) are commonly used to determine aerosol particle size distributions, particle morphology and particle composition to assist with source identification (Breed et al. 2002; Li et al. 2003; Moreno et al. 2003).

All SEM and EDS was carried out on a JEOL 6500F Scanning Electron Microscope at Victoria University of Wellington. Filter samples for analysis were prepared by taking a punch (10 mm diameter) from six glass fiber filters and attaching the punches with carbon glue to a $12.5 \mathrm{~mm}$ aluminum stub. Each sample was then sputter coated with approximately $15 \mathrm{~nm}$ of platinum to prevent sample charging during the measurement. The coated filters were then degassed using a PD3 Edwards Plate Degasser. Once prepared, filter samples were placed in a multi-stub holder and inserted into the electron microscope for analysis.

\subsection{Results}

\subsubsection{PAH emissions}

The emission of particle-phase PAHs was found to be highly dependent on wood burner operating condition for all wood species investigated. Whether or not the wood was softwood or hardwood also had an effect on PAH emission factors, with softwood combustion resulting in the greatest PAH emissions.

Table 7.1 presents individual and total PAH emission factors determined for softwood (pine) and hardwoods (macrocarpa and blue gum) in this study. It is evident from Table 7.1 that combustion of softwood produces the most significant quantities of particle-phase PAHs, even though total particulate emissions from hardwood were found to be greater. Softwood PAH emissions from the Pioneer Metro Eco burner were found to be very similar to an old, non-compliant wood burner studied previously (Ancelet et al. 2010). Total softwood particulate emissions were found to be $7.2 \pm 5.9 \mathrm{~g} / \mathrm{kg}$, in a similar range to previously reported results of $2.9-9.0 \mathrm{~g} / \mathrm{kg}$ (McDonald et al. 2000), $13.0 \pm 4 \mathrm{~g} / \mathrm{kg}$ (Hildemann et al. 1991) and more recently, $10.7 \pm 7.3 \mathrm{~g} / \mathrm{kg}$, in New Zealand (Ancelet et al. 2010). Total hardwood particulate emissions were found to be $13.3 \pm 7.9 \mathrm{~g} / \mathrm{kg}$, in agreement with previously reported 
results of 2.3-8.0 g/kg (McDonald et al. 2000) and $8.5 \pm 0.8 \mathrm{~g} / \mathrm{kg}$ (Schauer et al. 2001).

Table 7.1

PAH emission factors for softwood and hardwood

\begin{tabular}{|c|c|c|c|c|c|c|}
\hline \multirow{2}{*}{} & \multicolumn{2}{|c|}{$\begin{array}{c}\text { Softwood Emission Factors } \\
\text { (mg/kg wood) }\end{array}$} & \multicolumn{3}{c|}{ Hardwood Emission Factors (mg/kg } \\
& Ave & Median & Std dev & Ave & Median & Std dev \\
\cline { 2 - 7 } & & & & & & \\
\hline $\begin{array}{c}\text { Total Particulate } \\
\text { Emissions }\end{array}$ & 7233 & 5569 & 5940 & 13274 & 13449 & 7885 \\
\hline naphthalene & 0.3 & 0.1 & 0.7 & 0.1 & 0 & 0.1 \\
\hline acenaphthylene & 2.9 & 0.2 & 9 & 0.3 & 0.1 & 0.8 \\
\hline acenaphthene & 0.4 & 0 & 0.9 & 0 & 0 & 0.1 \\
\hline fluorene & 0.3 & 0.1 & 0.8 & 0.2 & 0 & 0.6 \\
\hline phenanthrene & 32 & 5 & 68 & 5 & 2 & 9 \\
\hline anthracene & 10 & 1 & 21 & 1 & 0.4 & 2 \\
\hline fluoranthene & 52 & 21 & 66 & 5 & 2 & 7 \\
\hline pyrene & 35 & 14 & 44 & 4 & 1 & 5 \\
\hline benzo[a]anthracene & 19 & 12 & 24 & 4 & 2 & 7 \\
\hline chrysene & 18 & 12 & 23 & 6 & 3 & 10 \\
\hline benzo[b]fluoranthene & 2 & 1 & 2 & 0.7 & 0.3 & 0.9 \\
\hline benzo[k]fluoranthene & 1 & 0.2 & 2 & 0.3 & 0 & 0.7 \\
\hline benzo[a]pyrene & 6 & 3 & 8 & 1 & 0.2 & 2 \\
\hline indeno[1,2,3-c,d]pyrene & 5 & 2 & 7 & 1 & 0.3 & 2 \\
\hline dibenzo[a,h]anthracene & 3 & 1 & 5 & 0.1 & 0 & 0.2 \\
\hline benzo[g,h,i]perylene & 2 & 2 & 3 & 0.6 & 0.2 & 0.8 \\
\hline Sum & 188.9 & 74.6 & & 29.3 & 11.5 & \\
\hline
\end{tabular}

The greater hardwood emissions in this study may be due to the hardwood having higher moisture content than the softwood. High moisture content will cause an appreciable amount of energy to be used in order to vaporise excess moisture, decreasing combustion efficiency and causing an increase in particulate smoke formation (Rogge et al. 1998). Although total hardwood particulate emissions were greater than those of softwood, particle-phase PAH emissions were greater from the combustion of softwood, a trend observed by Fine et al. (2002).

Benzo[a]pyrene $(\mathrm{BaP})$ is classified as a priority pollutant by the Ministry for the Environment in New Zealand due to its carcinogenic and mutagenic properties, making the analysis of $\mathrm{BaP}$ emissions from different wood types highly relevant. Table 7.2 presents average BaP emission factors for pine, macrocarpa, split blue gum and unsplit blue gum under each operating condition. Combustion of pine produced the highest quantities of $\mathrm{BaP}$ per $\mathrm{kg}$ wood burned, while blue gum produced the lowest quantities of BaP, suggesting that a reduction in the quantity of pine used in 
households around New Zealand could reduce ambient BaP concentrations. Wood burner operating condition was also found to have a large effect on BaP emissions. Except in the case of unsplit blue gum, start-up produced the highest quantities of BaP followed by low burn. High burn produced the lowest quantities of $\mathrm{BaP}$ in all cases except for unsplit blue gum. It is likely that the larger pieces of unsplit blue gum were not as significantly combusted during start-up as the smaller pieces, resulting in higher BaP emissions during main burn. Overall, unsplit blue gum had the lowest BaP emissions, suggesting that if larger pieces of wood are used during wood burner startup, $\mathrm{BaP}$ emissions can be significantly reduced from the highest emitting operating condition.

Table 7.2 BaP emission factors for all wood types studied under each operating condition

\begin{tabular}{|c|c|c|c|}
\hline Wood Type & \multicolumn{3}{|c|}{ Average BaP Emission Factor (mg/kg wood) } \\
\hline & Start-up & High burn & Low burn \\
\hline Pine & 9.9 & 0.6 & 8.7 \\
\hline Macrocarpa & 4.1 & 0.1 & 0.9 \\
\hline Split blue gum & 2.7 & $<0.1$ & 0.2 \\
\hline Unsplit blue gum & 0.3 & 0.5 & $<0.1$ \\
\hline
\end{tabular}

$\mathrm{PAH}$ emissions were further investigated by individual wood type and under each burner operating condition. The predominant PAH emissions from all wood types were phenanthrene (Phen), fluoranthene (Flt), pyrene (Pyr), benzo[a]anthracene (BaA) and chrysene (Chr). Figure 7.1 presents a box and whisker plot, representative of all the wood types studied, of particle-phase PAH emissions from the combustion of pine during start-up, high burn and low burn respectively. Inspection of Figure 7.1 reveals that $\mathrm{PAH}$ emissions are greatest during start-up and low burn when combustion of the wood is least efficient. Unsurprisingly, when combustion is most efficient (high burn), particulate PAH emissions are lowest. 


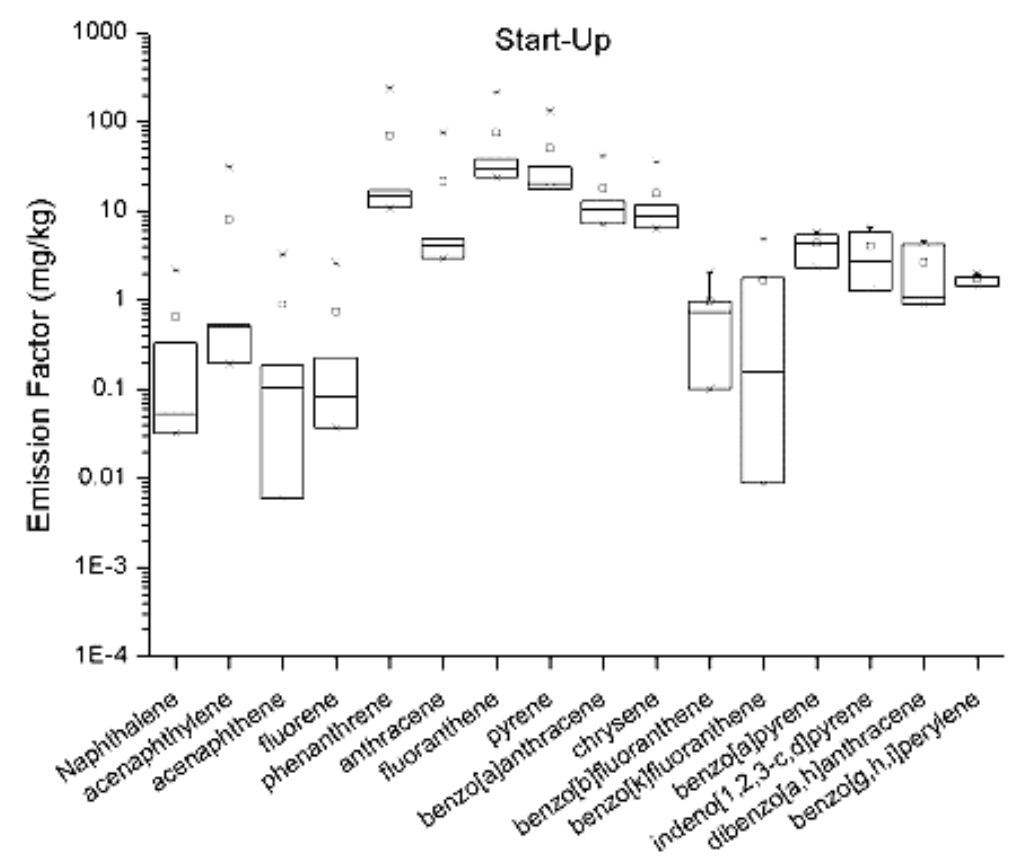

(a)

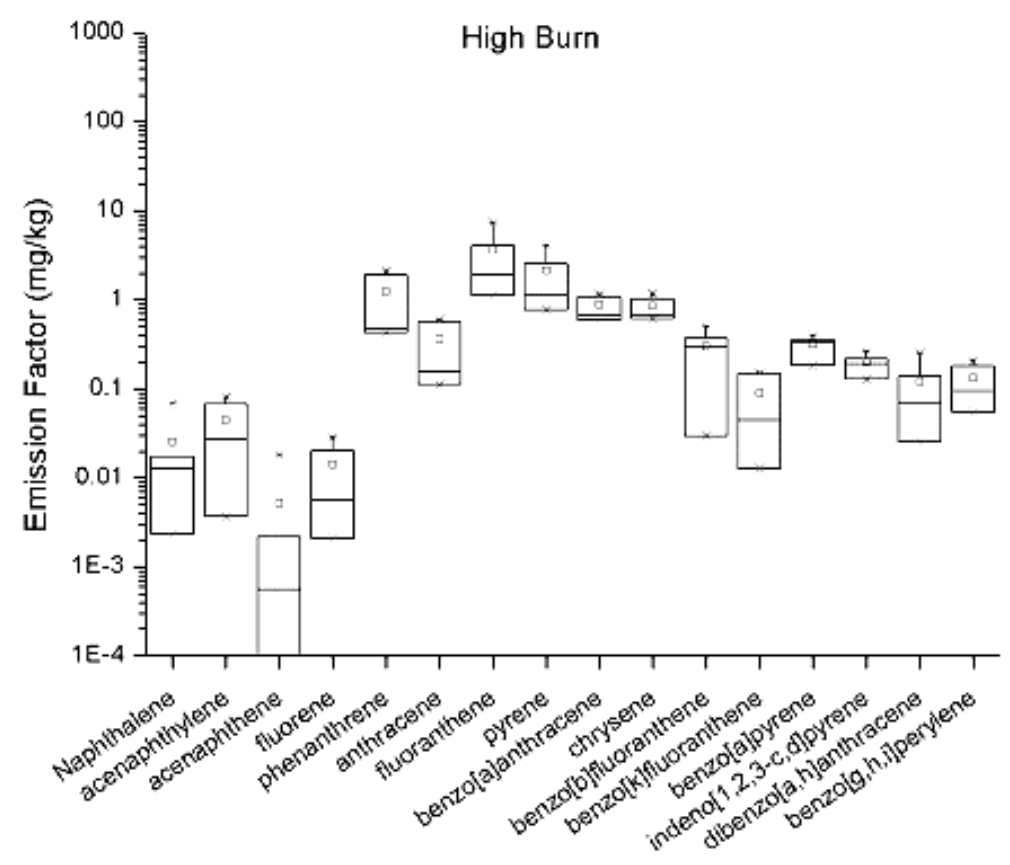

(b) 


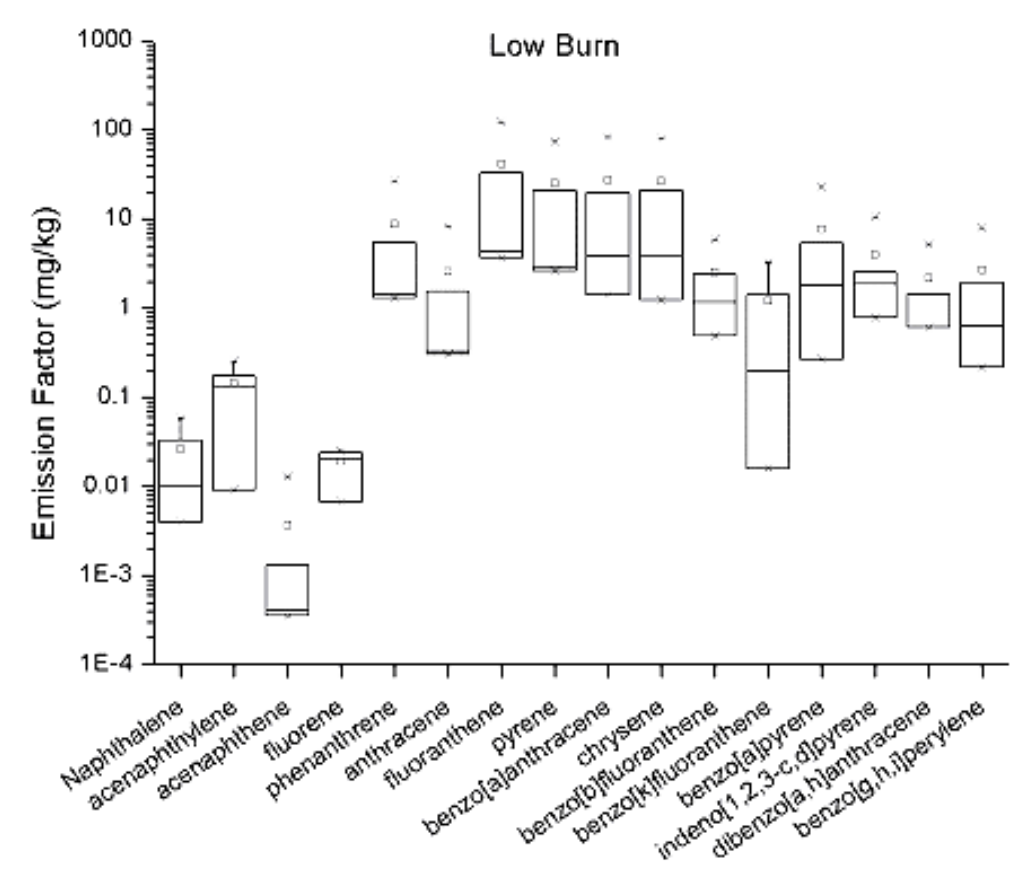

Figure 7.1 Representative box and whisker plot of PAH emissions from pine during (a) start-up $(\mathrm{n}=4)$, (b) high burn ( $\mathrm{n}=4)$ and (c) low burn $(\mathrm{n}=4)$

Particle-phase PAH emissions from macrocarpa followed a similar trend to PAH emissions from pine. Again, start-up and low burn produced the highest particlephase PAH emissions. Under all operating conditions PAH emissions were lower from the combustion of macrocarpa than from the combustion of pine.

$\mathrm{PAH}$ emissions from split and unsplit blue gum were similar to $\mathrm{PAH}$ emissions from macrocarpa. Split blue gum $\mathrm{PAH}$ emissions followed the trend of being highest during start-up and low burn, as seen with pine and macrocarpa. Unsplit blue gum, however, did not show a marked decrease in emissions under the high burn condition. This is likely due to the fact that larger pieces of wood will not be completely combusted as efficiently as smaller pieces of wood, resulting in more significant particulate PAH emissions under high burn than would normally be expected.

\subsubsection{Elemental emissions}

Elemental emissions were greatly affected by burner operating condition and largely unaffected by wood type and moisture content. The elemental composition of wood burner emissions was found to be dominated by hydrogen (a measure of the 
organic compound content) and black carbon (BC), which represents the elemental carbon (soot) component. Other important elemental constituents were $\mathrm{Na}, \mathrm{K}, \mathrm{S}$, and $\mathrm{Cl}$, which together contribute about $26 \%$ of the elemental mass. Table 7.3 presents a summary of the elemental emissions determined in this study. Potassium is typically used alongside $\mathrm{BC}$ as a marker for biomass burning and wood combustion in particular (Fine et al. 2002; Khalil and Rasmussen 2003). Iron observed in the samples was likely due to the composition of the wood burner (cast iron/steel) and ducting (galvanized steel) rather than the composition of the wood based on results discussed in the SEM analysis.

Table 7.3 Emission factors of elements (mg/kg wood) above their respective detection limits

\begin{tabular}{|c|c|c|c|c|c|c|}
\hline Element & \multicolumn{5}{|c|}{ Emission Factors (mg/kg wood) } & \\
\hline & Average & Max & Min & Median & Std dev & Number of samples above LOD \\
\hline $\mathrm{H}$ & 50 & 186 & 2 & 49 & 43 & 32 \\
\hline $\mathrm{BC}$ & 92 & 202 & 32 & 94 & 50 & 32 \\
\hline $\mathrm{Na}$ & 10 & 49 & 0 & 4 & 13 & 18 \\
\hline $\mathrm{Al}$ & 0.2 & 0.4 & 0 & 0.2 & 0.1 & 20 \\
\hline $\mathrm{Si}$ & 0.4 & 2 & 0.1 & 0.3 & 0.3 & 32 \\
\hline $\mathrm{P}$ & 2 & 31 & 0 & 0.1 & 6 & 16 \\
\hline$S$ & 4 & 14 & 0.9 & 3 & 3 & 32 \\
\hline $\mathrm{Cl}$ & 22 & 91 & 0.4 & 14 & 25 & 32 \\
\hline $\mathrm{K}$ & 14 & 55 & 0.2 & 6 & 16 & 32 \\
\hline $\mathrm{Ca}$ & 0.4 & 2 & 0 & 0.4 & 0.3 & 26 \\
\hline $\mathrm{Mn}$ & 0.2 & 1 & 0 & 0.1 & 0.3 & 14 \\
\hline $\mathrm{Fe}$ & 0.1 & 0.5 & 0 & 0.1 & 0.1 & 10 \\
\hline $\mathrm{Zn}$ & 0.3 & 3 & 0 & 0.1 & 0.5 & 16 \\
\hline $\mathrm{Ba}$ & 0.2 & 0.6 & 0 & 0.1 & 0.2 & 11 \\
\hline Sum & 196 & & & & & \\
\hline
\end{tabular}

The average total emission of elements was determined to be $196 \mathrm{mg} / \mathrm{kg}$ wood burned. The average and maximum emission factors for $\mathrm{Cl}$ were found to be 22 $\mathrm{mg} / \mathrm{kg}$ wood and $91 \mathrm{mg} / \mathrm{kg}$ wood, respectively, in good agreement with the results of Schauer et al. (2001) who reported average $\mathrm{Cl}$ emission of $110 \mathrm{mg} / \mathrm{kg}$ wood for eucalyptus. Although the elemental emission factors of Schauer et al. (2001) are larger than those obtained in our study, the relative composition of emissions was very similar to our results.

The relationship of various elemental components with wood type, moisture content and burner operating conditions were examined in detail. Figure 7.2 presents a plot of measured elemental mass emissions versus particulate matter emission 
concentrations during start-up and main burn (combination of particles collected during high and low burn).

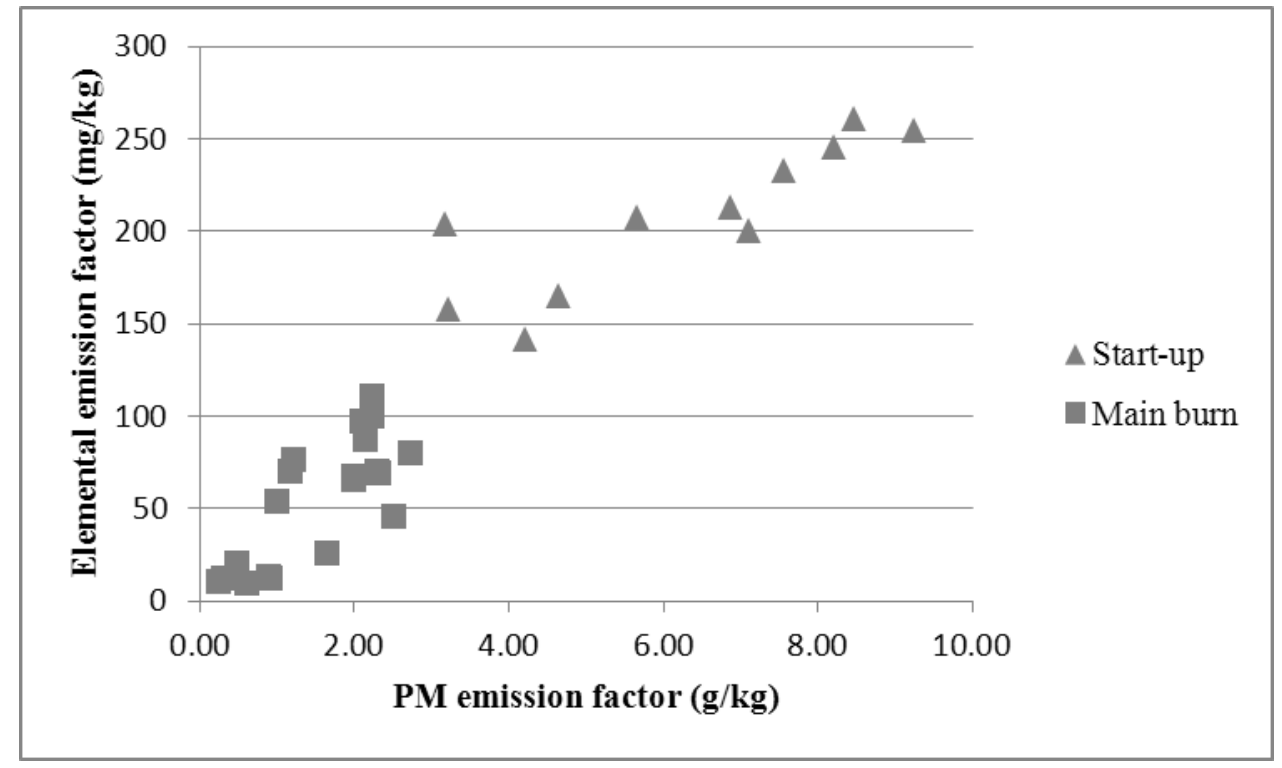

Figure 7.2 Elemental mass emissions versus particulate matter emission concentrations during start-up and main burn (27 blue gum, 6 macrocarpa)

Figure 7.2 shows that the highest elemental ( $\mathrm{H}$ and $\mathrm{BC}$ mainly) and particulate emissions occur during start-up conditions. This is most likely to be due to poor combustion during a cold start which produces greater amounts of smoke and pyrolysis products (largely made up of organic compounds formed during incomplete combustion). A plot of the inorganic emissions ( $\mathrm{H}$ and BC excluded; not shown) versus particulate matter concentration shows that contrary to the results for total elemental emissions, the inorganic elements of wood burner emissions were found to be highest during the main burn sequence. This is probably because woody organic material needs to be substantially combusted before inorganic elements are released in any quantity. The average inorganic component comprised approximately $4 \%$ of particulate matter emissions from this wood burner. Wood type was found to have very little impact on elemental emissions.

Wood moisture content is an important parameter in combustion. As such, elemental emissions from wood with $25 \%$ and $35 \%$ moisture content were investigated. Again, start-up produced higher elemental and particulate mass emissions than the main burn. Wood moisture content did not have a significant effect 
on emissions during start-up, but did have some effect on the particulate and elemental emissions during main burn conditions.

\subsubsection{Total carbon mass emissions}

Total carbon (TC) emissions and $\delta^{13} \mathrm{C}$ values were determined by EA/IRMS for samples of each wood type. Not only is TC concentration a useful measure of total carbonaceous species present in PM, but the TC concentration can be used in association with $\mathrm{BC}$ concentrations to determine the concentration of organic carbon (OC) in a sample. TC emissions are highest during start-up, in agreement with the IBA results for total particulate emissions discussed in the elemental emissions section. Wood type was found to play a significant role in TC emissions, with pine and unsplit blue gum producing the highest TC emissions. Stable isotope analysis of wood combustion emissions yielded $\delta^{13} \mathrm{C}$ values ranging from -22.01 to $-26.89 \%$, with no obvious differences due to wood type or operating conditions. The $\delta^{13} \mathrm{C}$ values presented are within the expected range for wood combustion emissions, but the use of $\delta^{13} \mathrm{C}$ values as a marker of wood smoke is not definitive since many other sources, including motor vehicle emissions, exhibit similar $\delta^{13} \mathrm{C}$ values (Okuda et al. 2002a; Lima et al. 2005; Jordan et al. 2006). This study does however add to the existing knowledgebase of $\delta^{13} \mathrm{C}$ values for wood smoke.

\subsubsection{SEM analysis}

Analysis of PM by SEM-EDS provides a useful measure of particle size and morphology, information that can be relevant in regards to possible health effects. SEM-EDS can also be used to identify the composition of individual particles to provide a better understanding of the inorganic species formed during the combustion process. Figures 7.3 and 7.4 present representative SEM images of pine combustion and blue gum combustion respectively. These images were obtained from filters collecting particulates during the start-up phase. The particle morphologies for both types of wood were found to be amorphous and a distinct difference in particle concentration was observed, with pine emissions completely coating the glass fiber filters and blue gum emissions sparsely distributed.

From the SEM images it can be seen that wood burner emissions tend to form large aggregates from much smaller spherules approximately 30 to $100 \mathrm{~nm}$ in 
diameter. The aggregates tend to 'coat' the glass fibers in the case of pine combustion or appear sporadically along a fiber in the case of blue gum combustion. Particle sizes due to combustion have previously been reported to occur in the $3-900 \mathrm{~nm}$ range, with peak concentrations in the 100 - $300 \mathrm{~nm}$ range (Kleeman et al. 1999; Hedberg et al. 2002). The masses of amorphous particles shown in Figures 7.3 and 7.4 are considered to be composed of a core of soot (elemental carbon) surrounded by a tarlike mass of organic compounds resulting from the incomplete combustion of wood.

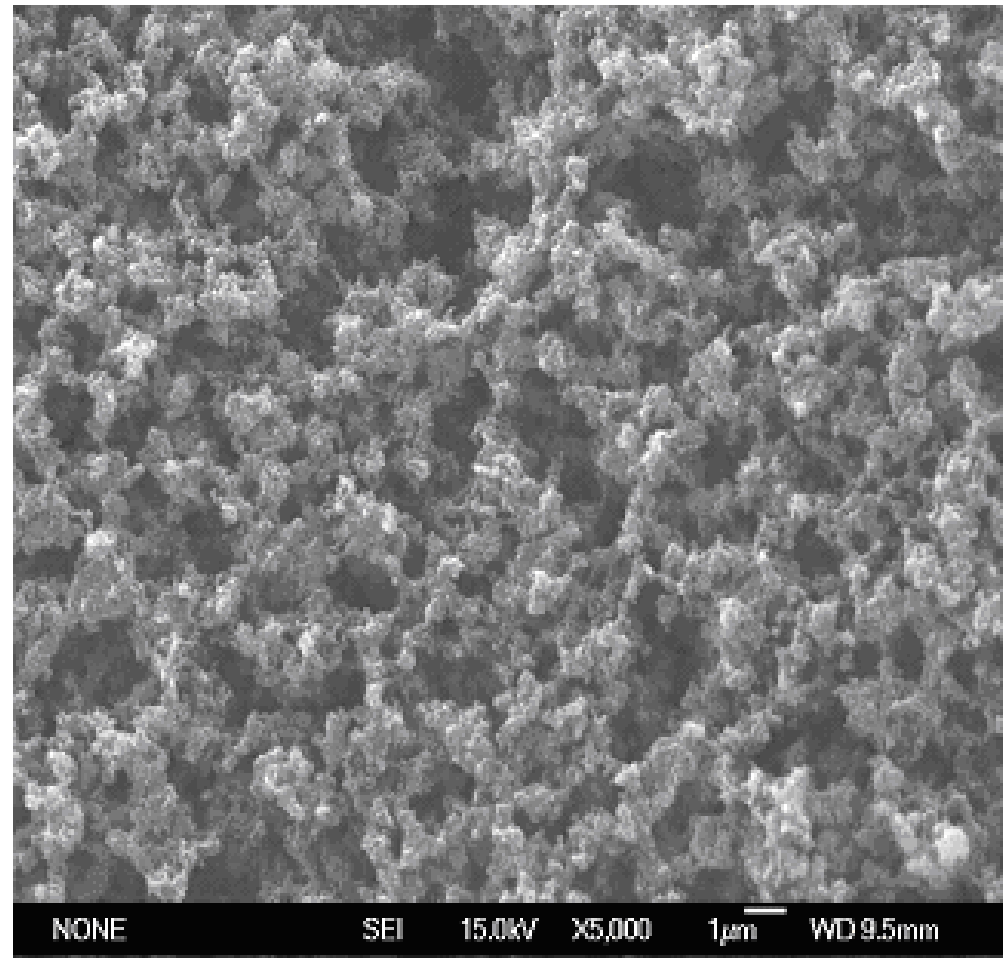

Figure 7.3 SEM image of particles from the combustion of pine during start-up 


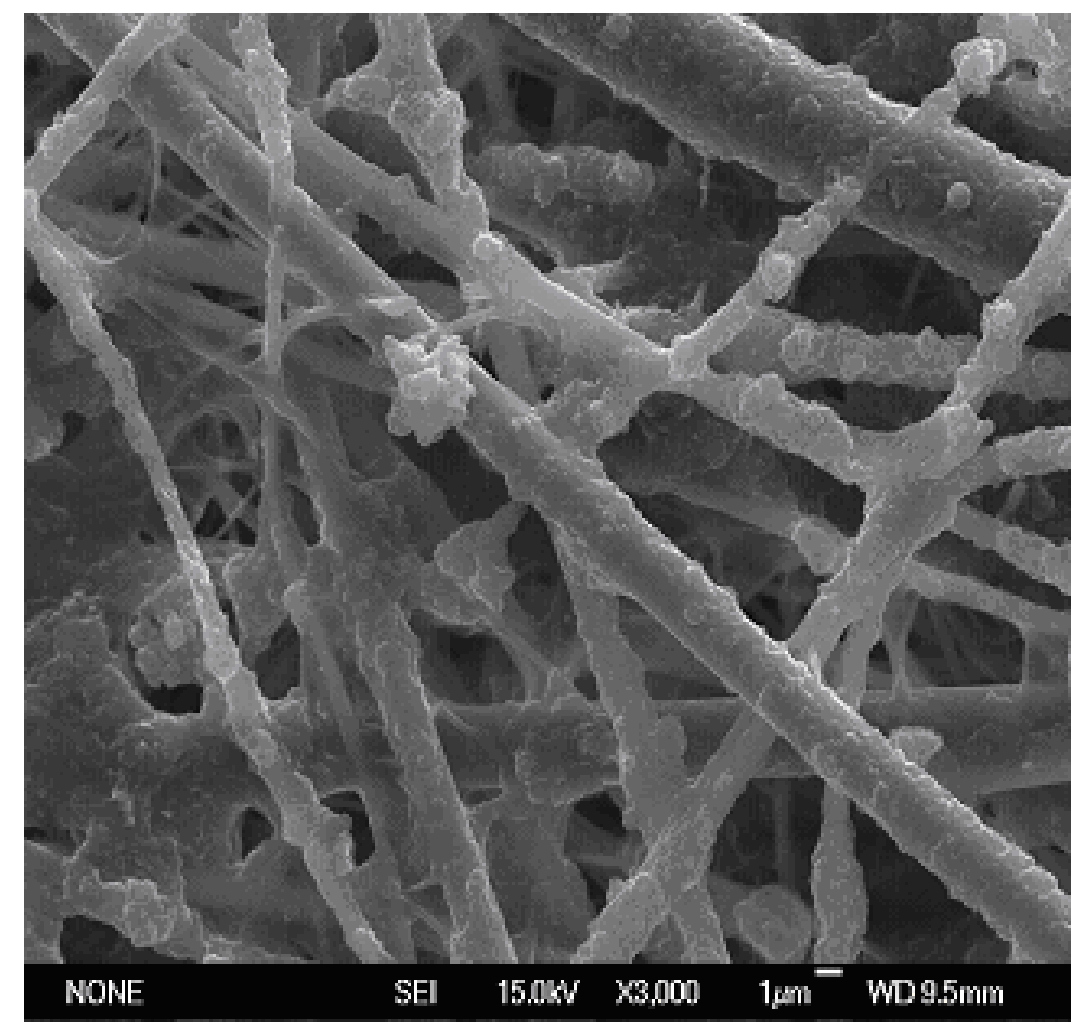

Figure 7.4 SEM image of particles from the combustion of blue gum during start-up

Interspersed amongst the carbonaceous particle mass collected on the filters were particles of different morphologies. In particular, when blue gum was used as a fuel and the wood burner was operated in high burn many inorganic fly ash particles were observed as shown in Figure 7.5. Figure 7.5 shows particles with platelet-like and crystalline morphologies, with EDS confirming that these larger, more welldefined particles are actually inorganic fly ash components. More accurately, EDS confirmed fly ash particles in wood smoke emissions were made up of $\mathrm{K}, \mathrm{Ca}, \mathrm{Cl}, \mathrm{S}, \mathrm{O}$ and $\mathrm{Ba}$, likely as the salts $\mathrm{KCl}, \mathrm{K}_{2} \mathrm{SO}_{4}, \mathrm{CaSO}_{4}$ and $\mathrm{BaSO}_{4}$ along with other minor components like nitrates, carbonates and magnesium salts.

An analysis of individual aerosol particles from biomass burning found that potassium salts and organic species were the predominant species in the smoke produced and were the most internally mixed (Li et al. 2003). Li et al. (2003) also found that $\mathrm{KCl}$ particles tend to occur in fresh smoke but reacted with sulfur and nitrogen species over time to produce $\mathrm{K}_{2} \mathrm{SO}_{4}$ and $\mathrm{KNO}_{3}$ particles. EDS analysis of pine emissions determined that a potassium sulfate salt made up the greatest proportion of fly ash, with very minor amounts of $\mathrm{Ca}$, Ba and Mg salts, in contrast with blue gum fly ash emissions discussed above. 


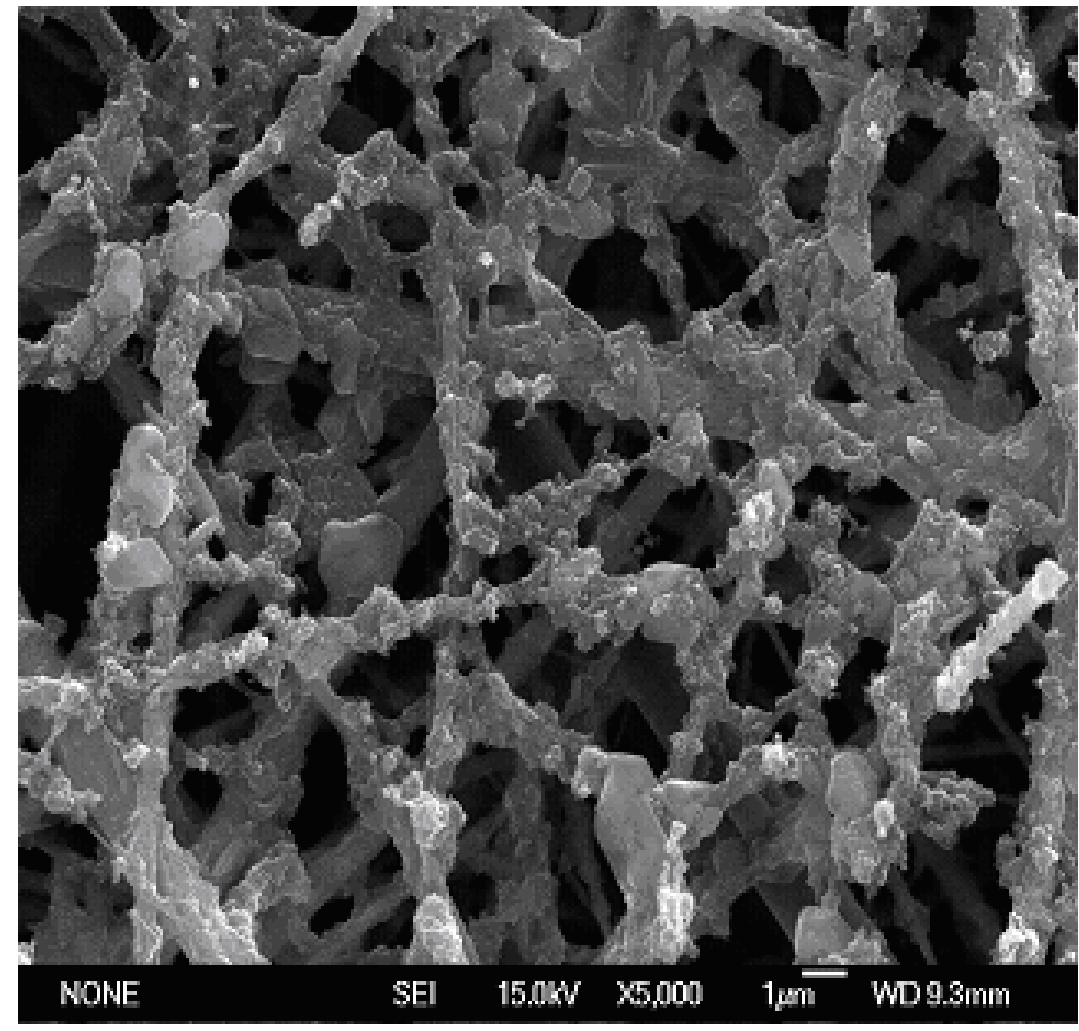

Figure 7.5 SEM image of various particle morphologies from the combustion of blue gum during high burn

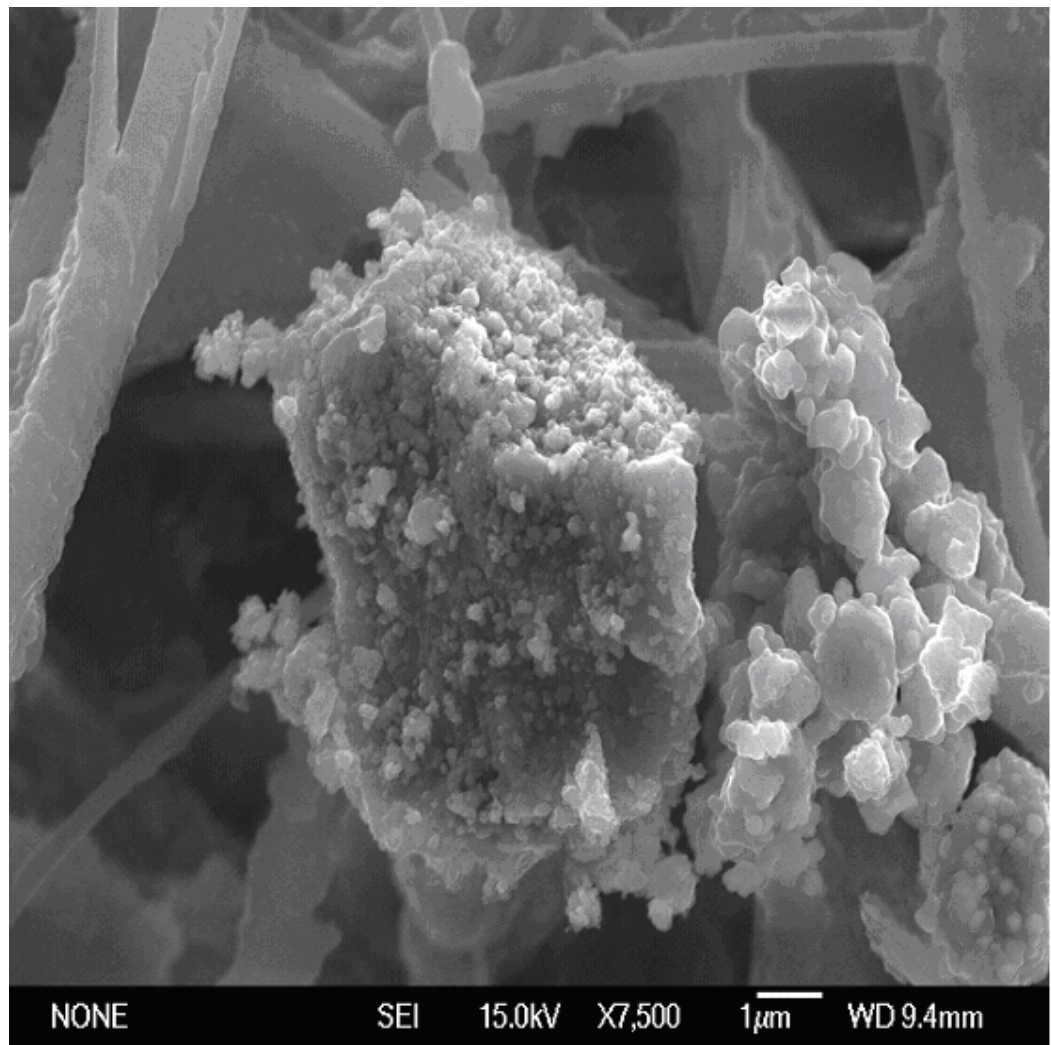

Figure 7.6 $\quad$ SEM image of a particle cluster made up of $\mathrm{Fe}, \mathrm{Cr}$ and $\mathrm{Ni}$ 
Particulate emissions from the combustion of wood are not always due to wood composition. Figure 7.6 presents a small amorphous cluster made up of iron, chromium and nickel, likely the result of some of the actual burner being emitted. Since elemental analysis did not reveal any significant quantities of chromium and nickel, it is likely that only small pieces of burner are emitted as discrete particle clusters and elemental analysis of a whole filter would not be sensitive enough to quantify a small amount of material at a discrete location.

\subsection{Conclusions}

This study characterised particulate matter emissions from a NES-compliant wood burner under varying burner conditions for the combustion of hardwood and softwood. Wood burner operating condition was found to have a significant influence on elemental and particle-phase PAH emissions, with start-up and low burn producing the highest concentrations. Although hardwood was found to produce the greatest $\mathrm{PM}_{10}$ emissions, softwood was found to produce significantly higher concentrations of particle-phase PAHs. In particular, $\mathrm{BaP}$ emissions were highest from the combustion of pine and significantly lower from the combustion of blue gum. Elemental analysis by IBA revealed that approximately 4\% of the PM emitted was composed of inorganic species. SEM analysis revealed that the particles tended to form large aggregates made up of small spherules, with amorphous morphologies. Interspersed amongst the carbonaceous species were inorganic, platelet-like particles primarily made up of $\mathrm{K}, \mathrm{Ca}, \mathrm{Cl}, \mathrm{S}, \mathrm{O}$ and $\mathrm{Ba}$, particularly for particles collected during the high burn cycle when combustion conditions were the most efficient.

On the basis of the results presented here, combustion of pine should be minimized due to the significantly greater PAH emissions of this wood versus the hardwoods studied here. Wood burners should be maintained in the high burn mode as much as possible in order to reduce particulate, elemental and PAH emissions. The results presented also demonstrate that PM concentration alone do not provide enough essential information about potential toxicity of the particles, since softwood combustion produced lower PM concentrations but dramatically higher PAH concentrations than hardwoods. The data presented in this study improves the current 
knowledge base for pollutants emitted from wood combustion and their emission factors.

\subsection{Acknowledgements}

The authors thank Auckland Regional Council for providing the wood burner emissions samples used in this study. TA thanks Andy Phillips for his assistance with the GC/MS and David Flynn for his assistance with the SEM-EDS measurements. This work was funded by the New Zealand Foundation for Research, Science and Technology under contract C05X0903. 


\section{Carbonaceous aerosols in an urban tunnel}

\subsection{Synopsis}

This chapter has been reproduced in full, with permission, as it appears in Atmospheric Environment, 2011. Ancelet, T., Davy, P. K., Trompetter, W. J., Markwitz, A. and Weatherburn, D. C., 2011. Carbonaceous aerosols in an urban tunnel. Atmospheric Environment, 45, 4463-4469.

Motor vehicle emissions can have a significant effect on local air quality, particularly in areas where heavy-duty vehicles are common. This study aimed to assess carbonaceous emissions from vehicles travelling through the Mount Victoria Tunnel in Wellington during peak traffic hours. Due to traffic congestion, PM emissions in the tunnel were high during these times and, correspondingly, particlephase PAH concentrations were also high. Diagnostic ratios suggested that diesel emissions were the most significant source of PAHs in the tunnel.

Total carbon (TC), organic carbon (OC) and elemental carbon (EC) were also quantified in this study using the thermal/optical IMPROVE_A and NIOSH protocols. In addition, TC was determined using elemental analysis - isotope ratio mass spectrometry (EA/IRMS) and black carbon (BC) was determined using light reflection. It was found that TC determined using EA/IRMS showed a direct 1:1 correlation with TC determined using the thermal/optical protocols. When TC concentrations from EA/IRMS are used in conjunction with BC concentrations it was suggested that OC concentrations could be determined from the difference between TC and BC. This was the first time that this result has been reported in the literature and provides a new method to determine the carbonaceous species present in a sample.

\subsection{Introduction}

Atmospheric pollution due to carbonaceous aerosol has important implications for global climate change and human health (Nel 2005; Russell and Brunekreef 2009; Pósfai and Buseck 2010). The primary components of carbonaceous aerosols are organic compounds such as polycyclic aromatic hydrocarbons (PAHs) and a 
multitude of other hydrocarbon compounds (Jacobson et al. 2000), elemental carbon (soot) as a by-product of incomplete combustion and inorganic carbon compounds (mainly carbonates). The emission of PAHs to atmosphere is of particular concern since many are known or suspected to be carcinogens and mutagens (IARC 1998). A variety of analytical methods are available for the quantification of organic compound content, soot and the total amount of carbon present in an aerosol sample.

In urban environments, motor vehicles are a major source of carbonaceous aerosols and despite improvements in diesel and gasoline engine technologies, motor vehicle pollution remains a significant issue for air pollution control in urban areas. Generally there are two methods used to investigate vehicular emissions; the dynamometer test and the real-world traffic study. While the dynamometer test allows for a relatively straightforward determination of engine-specific emission factors, it suffers from a number of serious drawbacks, including that it cannot account for a fleet of vehicles from a variety of makes, models and ages and an inability to account for other emissions associated with vehicles, like break and tire wear and resuspension of road dust (Thorpe and Harrison 2008). Consequently, dynamometer testing does not accurately reflect real-world particulate matter burden associated with vehicular emissions, making real-world traffic studies necessary. Real-world traffic studies are performed by sampling particulate matter (PM) at roadsides or in tunnels. Numerous real-world traffic studies have been performed in a variety of geographic locations with a variety of traffic volumes. (Kristensson et al. 2004; Chellam et al. 2005; Grieshop et al. 2006; Ho et al. 2009; Hung-Lung and Yao-Sheng 2009).

In this study, the carbonaceous content of PM in an urban road tunnel during peak hours in Wellington, New Zealand was analyzed for OC, EC and TC by thermal/optical methods, using both NIOSH and IMPROVE_A protocols, BC by light reflection and also TC by carbon isotope analysis. Particle-phase PAH concentrations from motor vehicles were quantified and single particle composition and morphology from motor vehicle emissions were examined by SEM-EDS. A comparison between the carbon analysis methods used to determine OC, EC, BC and TC concentrations is also presented. 


\subsection{Methodology}

\subsubsection{Sample collection}

The Mount Victoria Tunnel in Wellington, New Zealand is $623 \mathrm{~m}$ in length with two opposing traffic lanes aligned in a West-East direction. The tunnel has a grade of $1.7 \%$ as a vehicle travels East through the tunnel. Inside the tunnel there is a $1.5 \mathrm{~m}$ wide pedestrian footpath along one side that is raised $1.8 \mathrm{~m}$ above the eastern traffic lane. The cross-sectional area of the tunnel is $41 \mathrm{~m}^{2}$. Average traffic flow through the tunnel was approximately 2070-2300 vehicles per hour. Typical vehicle stream through the tunnel is 97-98 \% passenger cars and light commercial vehicles with a mix of diesel and gasoline fuelled engine types. The tunnel has a fully transverse ventilation system with two supply and two exhaust fans. All fans are turned on under normal operating conditions. Polluted air is removed from the tunnel by the two exhaust fans located at each side of the tunnel through vertical ventilation shafts, about $2.5 \mathrm{~m}$ in diameter.

PM emissions from the tunnel were collected with co-located Gent samplers, one employing a stacked filter unit (SFU) with coarse $\left(\mathrm{PM}_{10-2.5}\right)$ Nucleopore polycarbonate membrane filters $\left(0.8 \mu \mathrm{m}\right.$ pore size) and fine $\left(\mathrm{PM}_{2.5}\right)$ quartz fiber filters and the second employing a SFU with coarse Nucleopore polycarbonate membrane filters (0.8 $\mu \mathrm{m}$ pore size) and fine Teflon filters (Maenhaut et al. 1993). Co-located samplers were utilized in an effort to more accurately determine fine particle mass, since quartz fiber filters are well-known to display both positive and negative sampling artefacts, making the measurement of an accurate gravimetric mass difficult (Turpin et al. 2000; Mader et al. 2001; Viana et al. 2007). The samplers were placed in a ventilation outlet on the eastern side of the tunnel. The ventilation outlet house contains an exhaust fan for the tunnel and consists of a concrete bunker with dimensions 3m x 3m x 3m. The ventilation shaft from the tunnel below discharges directly into the fan room where the foul air is extracted and discharged to the atmosphere by the fan. The sampler was therefore sampling well-mixed air extracted directly from the tunnel itself. Sampling was carried out during peak traffic flows on weekdays, either between 04:00 and 08:00 or between 14:00 and 18:00, in an effort to sample maximum particulate matter concentrations in the tunnel exhaust stream. The monitoring program ran from December 2008 to March 2009 and a total of 33 PM$_{2.5}$ and $\mathrm{PM}_{10-2.5}$ filters were collected over the sampling period. 


\subsubsection{Carbon analysis}

Filter punches (11 mm diameter) from all of the loaded fine quartz filters were analyzed for OC and EC in the laboratory of Philip Hopke at Clarkson University using both the NIOSH 5040 and IMPROVE_A protocols. The NIOSH protocol was performed using a Sunset Laboratory OC/EC Analyzer and the IMPROVE_A protocol was performed using a DRI Model 2001. The IMPROVE_A and NIOSH protocols utilize the same thermal evolution method, only their temperature and optical monitoring protocols differ. The IMPROVE_A and NIOSH protocols have previously been described in detail and comparisons of the OC, EC and TC values obtained using each protocol on PM samples from the same filter have been reported (Birch and Cary 1996; Chow et al. 2001, 2007a).

Black carbon was measured on all 33 fine filters using a M43D Digital Smoke Stain Reflectometer. Since the amount of light absorption by the filter is directly proportional to the amount of $\mathrm{BC}$ present on the filter, Equation 1 can be used to obtain BC concentrations ( $\mu \mathrm{g} \mathrm{cm}^{-2}$ ) for quartz fiber filters (W. Maenhaut, pers. comm.)

$$
\mathrm{BC}=\left[1000 \mathrm{x} \log \left(\mathrm{R}_{\text {blank }} / \mathrm{R}_{\text {sample }}\right)+2.39\right] / 45.8
$$

Where $\mathrm{R}_{\text {blank }}$ is the average reflectance for a series of blank filters with a value close to 100 (indicating $100 \%$ reflectance) and $\mathrm{R}_{\text {sample }}$ is the reflectance of a loaded filter. The constants 2.39 and 45.8 were derived from a series of 100 quartz fiber filters which served as secondary standards for which the BC loadings $\left(\mu \mathrm{g} \mathrm{cm}^{-2}\right.$ ) had been determined by Prof. Dr. M. O. Andreae relative to standards that were prepared by collecting burning acetylene soot on filters and determining the mass concentrations gravimetrically. Filter blanks were analyzed every 6 samples to ensure reflectance measurements returned to 100 .

In addition to the TC results from the thermal methods, TC was also determined on all fine filters using elemental analysis-isotope ratio mass spectrometry (EA/IRMS). The isotope analysis was performed by the Stable Isotope Laboratory at the National Isotope Centre in Gracefield, New Zealand using a GVI IsoPrime mass spectrometer with leucine $\left(\delta^{13} \mathrm{C}=-22.7 \%\right.$ ) as a working standard for $\delta^{13} \mathrm{C}$ values and TC concentrations. The protocol used for TC and $\delta^{13} \mathrm{C}$ determination using EA/IRMS involved completely combusting loaded filters at $850{ }^{\circ} \mathrm{C}$ under continuous 
flow, with the $\mathrm{CO}_{2}$ produced analyzed by IRMS to yield $\delta^{13} \mathrm{C}$ values. TC concentrations were determined using MS signal intensities of different weights of leucine. Since the percentage of carbon in leucine is known, the mass of carbon in the standard was calculated for each different weight of leucine used and a standard curve was generated. The standard curve produced was then used to calculate the mass of carbon on the loaded filters.

\subsubsection{PAH analysis}

The concentrations of sixteen PAHs classified as priority pollutants by the United States Environmental Protection Agency (USEPA) were determined using thermal desorption GC/MS (TD/GC/MS). The analysis of PAHs in particulate matter using thermal desorption has previously been shown to provide comparable results to USEPA method TO-13A in the analysis of a NIST standard reference material (SRM1649a) (Waterman et al. 2000; Falkovich and Rudich 2001; Bates et al. 2008).

Prior to analysis, a small sample was taken from each filter using a punch (11 mm diameter). The filter punches were then placed into individual, empty stainless steel cups (Frontier Lab) and spiked with naphthalene- $d_{8}$, phenanthrene- $d_{10}$ and pyrene- $d_{12}$ obtained from Aldrich. PAHs were not detected in the empty cups or on blank filters. Once spiked, the samples were placed in a Frontier Lab Auto-Sampler in preparation for desorption. Thermal desorption was performed using a Frontier Lab Double-Shot Pyrolyzer in the single shot mode at $300{ }^{\circ} \mathrm{C}$ for 3 minutes in order to avoid organic compound degradation. The pyrolyzer was directly interfaced to a GC/MS unit (Agilent 7890A GC and 5975C MSD) with helium as the carrier gas. The desorbed analytes were trapped on a cryogenically cooled Frontier Lab Micro-Jet Cryo Trap until desorption was complete. The completeness of desorption was confirmed to be greater than $99 \%$ by a repeated desorption of an already analyzed filter.

Upon completion of the desorption step, the cooled analytes were released to an Agilent HP-5 column (30 m x $320 \mu \mathrm{m}$ x $0.25 \mu \mathrm{m}$ ). The GC oven was held at $80^{\circ} \mathrm{C}$ for $3 \mathrm{~min}$, ramped at $6^{\circ} \mathrm{C} / \mathrm{min}$ to $150^{\circ} \mathrm{C}$, at $3{ }^{\circ} \mathrm{C} / \mathrm{min}$ to $260^{\circ} \mathrm{C}$ and at $6^{\circ} \mathrm{C} / \mathrm{min}$ to 280 ${ }^{\circ} \mathrm{C}$ when it was held constant for $12 \mathrm{~min}$. The MS detector was operated in Selected Ion Monitoring (SIM) mode. PAHs were quantified using relative response ratios determined from certified authentic and isotopically labeled internal standards. A 
standard PAH solution used for calibration was obtained from Supelco and was used as received (TCL Polynuclear Aromatic Hydrocarbons Mix). Adequate linearity $\left(r^{2}\right.$ $\geq 0.98$ ) was obtained from calibrations for all sixteen PAHs. Analysis of multiple punches from the same filter revealed that PAH concentrations between filter punches varied by less than two percent overall. This was repeated for a number of filters which yielded the same result, verifying that the filter punches were highly representative of the filter as a whole.

\subsubsection{Scanning electron microscopy (SEM)}

Scanning electron microscopy (SEM) and energy dispersive spectroscopy (EDS) are commonly used to determine aerosol particle size distributions, particle morphology and particle composition to assist with source identification (Breed et al. 2002; Li et al. 2003; Moreno et al. 2003).

All SEM and EDS was carried out on a JEOL 6500F Scanning Electron Microscope at Victoria University of Wellington. Filter samples for analysis were prepared by taking a punch (11 mm diameter) from ten fine filters and two coarse filters and attaching the punches with carbon glue to a $12.5 \mathrm{~mm}$ aluminum stub. Each sample was then sputter coated with approximately $15 \mathrm{~nm}$ of platinum to prevent sample charging during the measurement. The coated filters were then degassed using a PD3 Edwards Plate Degasser. Once prepared, filter samples were placed in a multistub holder and inserted into the electron microscope for analysis. Images were collected over a constant range of magnifications in order to facilitate ease of comparison between samples.

\subsection{Results and discussion}

\subsubsection{PM, OC, EC and TC}

The average fine particle $\left(\mathrm{PM}_{2.5}\right)$ concentration in the Mount Victoria Tunnel was found to be $67.6 \pm 19.4 \mu \mathrm{g} \mathrm{m} \mathrm{m}^{-3}$. Average OC, EC and TC concentrations determined by NIOSH and IMPROVE are displayed in Table 8.1 along with OC/EC ratios and the percentage of each component relative to fine particle concentration. From Table 8.1 it is evident that the OC, EC and TC concentrations determined using the IMPROVE and NIOSH protocols are very similar. In particular, differences in TC 
concentrations were not statistically significant between NIOSH and IMPROVE, in good agreement with other studies (Bae et al., 2009; Cheng et al., 2011; Chow et al., 2001).

Table 8.1 Average OC, EC and TC concentrations (and associated standard deviations in parentheses) obtained from NIOSH and IMPROVE thermal/optical protocols along with OC/EC ratios and percentage of each component relative to fine particle concentration

\begin{tabular}{ccc}
\hline & NIOSH & IMPROVE \\
\hline OC $\left(\mu \mathrm{g} \mathrm{m}^{-3}\right)$ & $30.9(8.9)$ & $21.7(6.9)$ \\
$\mathrm{EC}\left(\mu \mathrm{g} \mathrm{m}^{-3}\right)$ & $18.6(8.0)$ & $21.3(6.2)$ \\
$\mathrm{TC}\left(\mu \mathrm{g} \mathrm{m}^{-3}\right)$ & $49.5(16.6)$ & $43.0(15.8)$ \\
OC/EC & 1.7 & 1.4 \\
$\% \mathrm{OC}$ & 46 & 32 \\
$\% \mathrm{EC}$ & 27 & 31 \\
$\% \mathrm{TC}$ & 73 & 63
\end{tabular}

A plot of TC concentrations determined using IMPROVE versus TC concentrations determined using NIOSH is presented in Figure 8.1.

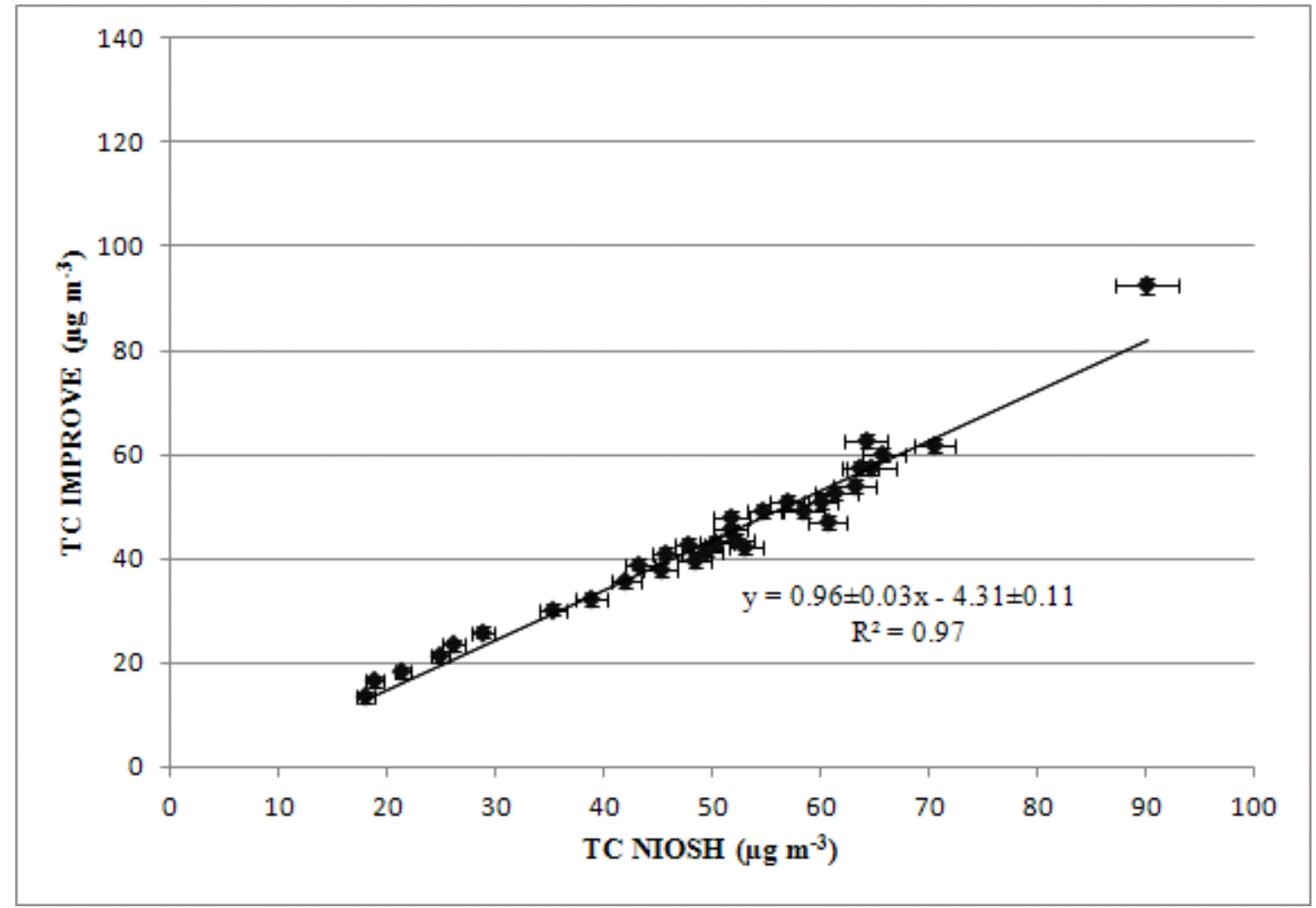


Figure 8.1 Plot of total carbon concentrations $\left(\mu \mathrm{g} \mathrm{m}^{-3}\right)$ determined using IMPROVE versus total carbon concentrations $\left(\mu \mathrm{g} \mathrm{m}^{-3}\right)$ determined using NIOSH

TC concentrations determined by IMPROVE and NIOSH shows excellent correlation and within measurement uncertainties the slope equals unity. The intercept, however, was not found to be zero within the measurement uncertainties as would be expected for the methods to be equivalent. It is likely that the different instruments used for the NIOSH and IMPROVE protocols in this study contributed to the small difference in TC concentrations observed. It is also possible that the lower temperature plateau for the IMPROVE method contributed to a TC underestimation relative to NIOSH.

The NIOSH protocol OC/EC ratio was 1.7, while the IMPROVE protocol OC/EC ratio was 1.0. The higher OC/EC ratio from the NIOSH protocol compared to the IMPROVE protocol is not surprising, since the NIOSH protocol incorporates a higher temperature organic carbon final heating step that is known to also incorporate some EC, therefore increasing the concentration of carbon that is attributed to OC (Chow et al. 2001). The low OC/EC ratios in this study are indicative of motor vehicles and suggest that diesel emissions are the largest contributor since the OC/EC ratios in this study are closer to those reported for diesel vehicles $(0.28-0.92)$ than to those reported for gasoline vehicles (> 2) (Cadle et al. 1999; Gillies and Gertler 2000; Allen et al. 2001; Watson et al. 2001). OC and EC were found to make up more than $70 \%$ of PM mass in this study. Fine particle OC and EC concentrations in this study were found to be higher than those in Taiwan in the Chung-Liao Tunnel $\left(10 \mu \mathrm{g} \mathrm{m}^{-3}\right.$ and $20 \mu \mathrm{g} \mathrm{m}^{-3}$ for OC and EC respectively) and Xueshan Tunnel (23.9 $\mu \mathrm{g} \mathrm{m}^{-3}$ and $18.9 \mu \mathrm{g} \mathrm{m}^{-3}$ for OC and EC respectively), but much lower than in the Zhujiang Tunnel in China (53 $\mu \mathrm{g} \mathrm{m}^{-3}$ and $94 \mu \mathrm{g} \mathrm{m}^{-3}$, for OC and EC respectively) (Huang et al. 2006; Hung-Lung and Yao-Sheng 2009; Zhu et al. 2010). The high OC and EC concentrations found in the Mount Victoria Tunnel compared with the tunnels in Taiwan is likely due to the fact that the Mount Victoria Tunnel is an urban tunnel, where traffic flow is significantly impeded during rush hour, as opposed to a freeway tunnel like those in Taiwan. Compared to the Zhujiang Tunnel in China though, OC and EC concentrations in the Mount Victoria Tunnel are low, since the urban Zhujiang Tunnel experiences higher traffic volumes and a significantly higher proportion of that traffic is made up of diesel vehicles. The lower OC/EC ratios in the Chung-Liao and Zhujiang tunnels when compared to the Mount Victoria Tunnel is not 
surprising, since the vehicle fleet travelling through those tunnels was made up of a larger proportion of diesel vehicles.

Using light reflection, the average BC concentration was determined to be $35.1 \pm 9.8 \mu \mathrm{g} \mathrm{m}^{-3}$. BC concentrations in this study were found to correlate extremely well with EC concentrations from both the NIOSH and IMPROVE protocols ( $r=0.96$ and 0.90 respectively). The determination of $\mathrm{BC}$ on filters using light reflection is non-destructive and allows for near real-time operation, but since some high molecular weight organic species are known to also absorb light, BC concentrations from light reflection are likely to overestimate the "elemental" carbon present in a sample (Andreae and Gelencśer 2006). Application of paired t-tests revealed that BC concentrations determined by light reflection were not significantly different than EC concentrations determined using thermal/optical procedures.

Average TC concentrations using EA/IRMS were found to be $51.0 \pm 16.7 \mu \mathrm{g}$ $\mathrm{m}^{-3}$, in excellent agreement with the TC results obtained using the NIOSH protocol and not significantly different than the TC results obtained using IMPROVE. Figure 8.2 presents a plot of TC concentrations determined using NIOSH and IMPROVE versus TC concentrations determined using EA/IRMS. From Figure 8.2 it is clear that within measurement uncertainties the slope equals unity and the intercept is zero when TC from EA/IRMS is compared with TC determined using the NIOSH protocol. This result is extremely interesting, since to the best of our knowledge no direct comparison between TC determined using thermal/optical methods and EA/IRMS has been reported and the direct, 1:1 correlation between the NIOSH protocol and EA/IRMS demonstrates that EA/IRMS can be used as an accurate measure of TC in air particulate matter samples. Also, since BC determined using optical reflectance has been shown in this work to produce comparable results to EC concentrations from thermal/optical methods, the combination of TC determined using EA/IRMS and BC determined using light reflection can be used to accurately assess carbonaceous species present in a sample. OC determined using this technique would then be described as $\mathrm{OC}=\mathrm{TC}-\mathrm{BC}$. $\mathrm{OC}$ concentrations calculated using this method correlated very well with OC concentrations determined using NIOSH and IMPROVE ( $r=0.91$ and 0.88 respectively) and the average OC concentration was determined to be $15.9 \pm 9.8 \mu \mathrm{g} \mathrm{m}^{-3}$. As an alternate methodology to thermal/optical reflectance methods, by combining EA/IRMS with light reflectance, it is possible to 
determine TC, EC and OC accurately with unattended operation and low cost per filter.

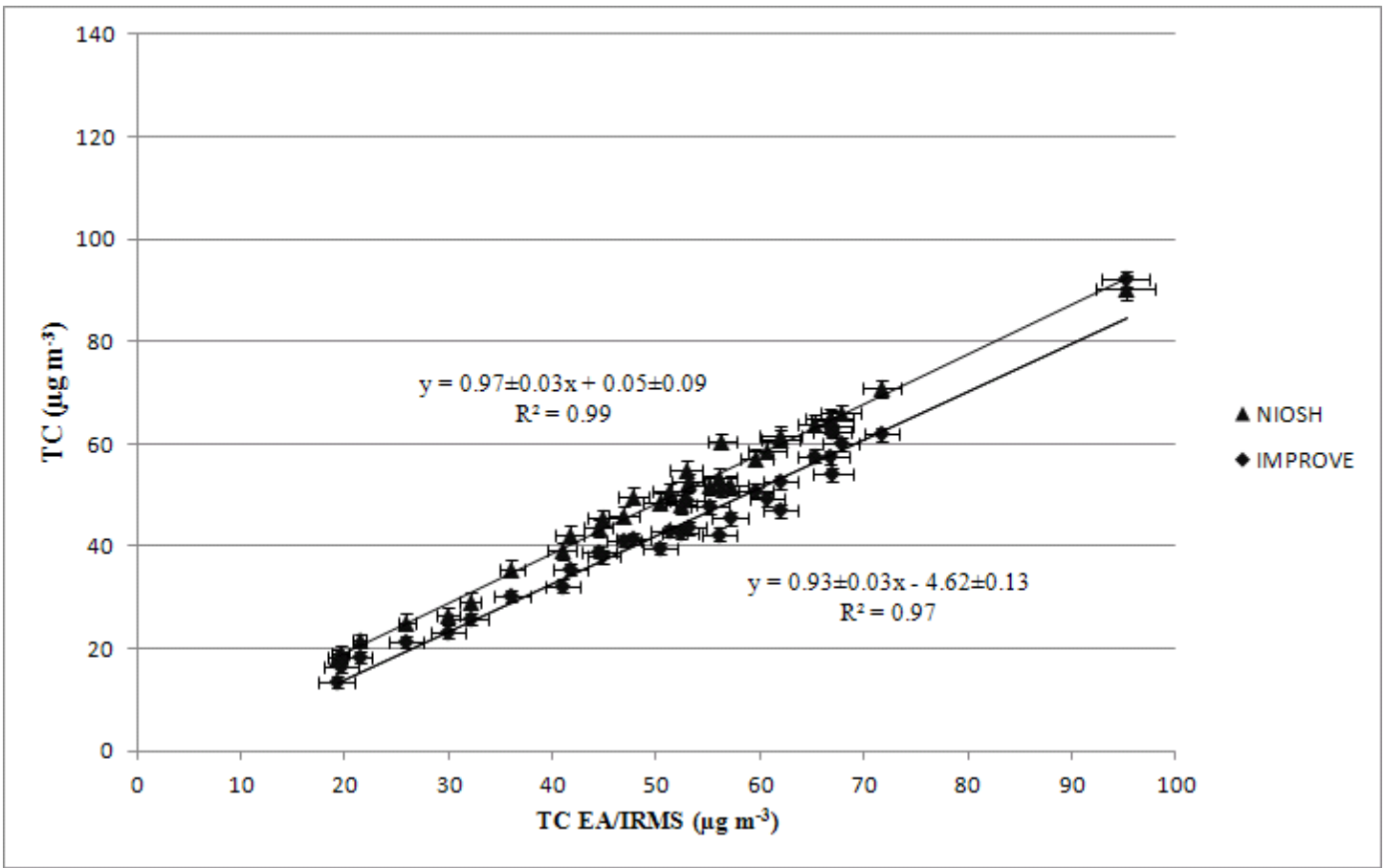

Figure 8.2 Plot of total carbon concentrations $\left(\mu \mathrm{g} \mathrm{m}^{-3}\right)$ determined using NIOSH and IMPROVE versus total carbon concentrations $\left(\mu \mathrm{g} \mathrm{m}^{-3}\right)$ determined using EA/IRMS

Stable isotope analysis of motor vehicle emissions yielded $\delta^{13} \mathrm{C}$ values ranging from $-24.7 \pm 0.2$ to $-28.3 \pm 0.2 \%$ with an average value of $-25.9 \pm 0.8 \%$, in agreement with previously reported ranges of -12.9 to $-26.6 \%$ (Okuda et al., 2002b), -22.6 to $-25.4 \%$ (O’Malley et al., 1994) and -26 to $-28 \%$ (Widory, 2006). The range in $\delta^{13} \mathrm{C}$ values is not unexpected, since motor vehicle emissions have been shown to display a large inter-species variation in $\delta^{13} \mathrm{C}$ values (Okuda et al., 2002a; Okuda et al., 2002b). While the $\delta^{13} \mathrm{C}$ values obtained in this study are within the expected range for motor vehicles, the use of $\delta^{13} \mathrm{C}$ values as a marker of motor vehicle emissions is not definitive since other sources, including wood smoke, exhibit similar $\delta^{13} \mathrm{C}$ values (Okuda et al. 2002a; Okuda et al. 2002b; Lima et al. 2005; Jordan et al. 2006).

\subsubsection{PAH emissions}

Average particle-phase PAH concentrations in the Mount Victoria Tunnel are presented in Table 8.2. The total average concentration of the 16 PAHs analyzed for

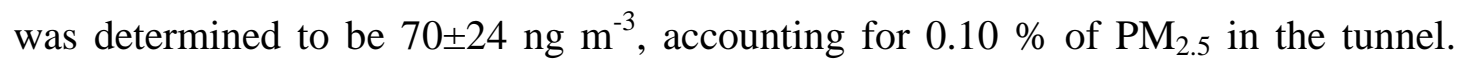


Fluoranthene and pyrene are the most abundant particle-phase PAHs in the tunnel, with concentrations ranging from 8.7 to $21.1 \mathrm{ng} \mathrm{m}^{-3}$ and 12.1 to $25.7 \mathrm{ng} \mathrm{m}^{-3}$ respectively. Fluroanthene and pyrene have also been reported to be the most abundant particle-phase PAHs in tunnels in Hong Kong (20.0 to $42.2 \mathrm{ng} \mathrm{m}^{-3}$ and 26.5 to $52.3 \mathrm{ng} \mathrm{m}^{-3}$ respectively) and Marseille (45 and $23.3 \mathrm{ng} \mathrm{m}^{-3}$ respectively) (El Haddad et al. 2009; Ho et al. 2009). The results presented in Table 8.2 also demonstrate that $\mathrm{PAH}$ emissions in the tunnel are enriched in low molecular weight PAHs (those that have four rings or less). Previous studies have suggested that diesel vehicle emissions are enriched in low molecular weight PAHs $(\leq 4$ rings), while emissions from gasoline vehicles are enriched in high molecular weight PAHs, indicating diesel emissions in the Mount Victoria Tunnel contribute more significantly to total PAH concentration compared to gasoline-fueled vehicles (Schauer et al. 1999; Schauer et al. 2002; Zielinska et al. 2004).

Table 8.2 Average particle-phase PAH concentrations (and associated standard deviations in parentheses) obtained from TD/GC/MS

\begin{tabular}{|c|c|c|}
\hline PAH & No. of rings & Concentration $\left(\mathrm{ng} \mathrm{m}^{-3}\right)$ \\
\hline Naphthalene & 2 & $0.8(0.4)$ \\
\hline Acenaphthylene & 3 & $0.5(0.3)$ \\
\hline Acenaphthene & 3 & $0.4(0.2)$ \\
\hline Fluorene & 3 & $0.4(0.2)$ \\
\hline Phenanthrene & 3 & $4.8(1.2)$ \\
\hline Anthracene & 4 & $2.1(0.9)$ \\
\hline Fluoranthene & 4 & $14.1(4.4)$ \\
\hline Pyrene & 4 & $18.2(6.0)$ \\
\hline Benzo[a]anthracene & 4 & $10.5(3.2)$ \\
\hline Chrysene & 5 & $0.9(0.4)$ \\
\hline Benzo[b]fluoranthene & 5 & $0.9(0.3)$ \\
\hline Benzo[k]fluoranthene & 5 & $1.7(0.5)$ \\
\hline Benzo[a]pyrene & 6 & $1.5(0.7)$ \\
\hline Indeno[1,2,3-c,d]pyrene & 5 & $0.2(0.1)$ \\
\hline Dibenz[a,h]anthracene & 6 & $3.2(1.1)$ \\
\hline Benzo[g,h,i]perylene & & \\
\hline
\end{tabular}




\begin{tabular}{l|l|r} 
Sum & $70(24)$ \\
\hline
\end{tabular}

Another indication that diesel emissions contribute more than gasoline emissions to the total PAH concentration can be found in the ratio of indeno[1,2,3c,d]pyrene to the sum of indeno[1,2,3-c,d]pyrene and benzo[ghi]perylene (I[cd]P/(I[cd]P+B[ghi]P), which has been reported to be 0.18 for gasoline and 0.37 for diesel (Gogou et al. 1996). In the Mount Victoria Tunnel this ratio was 0.32, indicating a significant contribution from diesel vehicles in agreement with the enrichment of low molecular weight PAHs.

Benzo[a]pyrene (BaP) is a PAH that is of particular interest due to its role as a probable human carcinogen (IARC 1998). The average particle-phase BaP concentration in the Mount Victoria Tunnel was determined to be $1.7 \pm 0.5 \mathrm{ng} \mathrm{m}^{-3}$, within the range reported for severe winter pollution episodes in two major New Zealand cities (Cavanagh et al. 2009).

\subsubsection{SEM analysis}

Relatively few studies have performed single particle characterization and morphology analysis of motor vehicle emissions (Berube et al. 1999; Paoletti et al. 1999; Dye et al. 2000; Kocbach et al. 2005). Similarly to these studies, PM in the Mount Victoria Tunnel was found to consist largely of carbonaceous material and individual particles exhibited amorphous morphologies. Motor vehicle emissions formed large aggregates from much smaller spherules approximately 30 to $100 \mathrm{~nm}$ in diameter. Figure 8.3 presents a representative SEM image of agglomerated carbonaceous particles. The particle aggregates tended to appear sporadically along the quartz fibers.

Interspersed amongst the carbonaceous particle mass on the filters were particles made up of $\mathrm{Ca}, \mathrm{S}, \mathrm{Fe}, \mathrm{P}, \mathrm{Si}, \mathrm{Al}, \mathrm{Cl}, \mathrm{Na}, \mathrm{O}$ and $\mathrm{Zn}$. These elements tended to be present in low quantities compared to carbon, but their presence is not surprising since these elements are known to be present in motor vehicle exhaust or produced through motor vehicle actions like brake wear, tire wear and road dust re-suspension (Thorpe and Harrison 2008). Also apparent from the SEM-EDS analysis is the presence of sea salt inside the tunnel, most likely due to the presence of marine aerosol in tunnel intake air. SEM-EDS analysis of particulate matter from the Mount 
Victoria Tunnel revealed that PM consisted largely of carbonaceous material, in agreement with the carbon analysis discussed in Chapter 8.4.1.

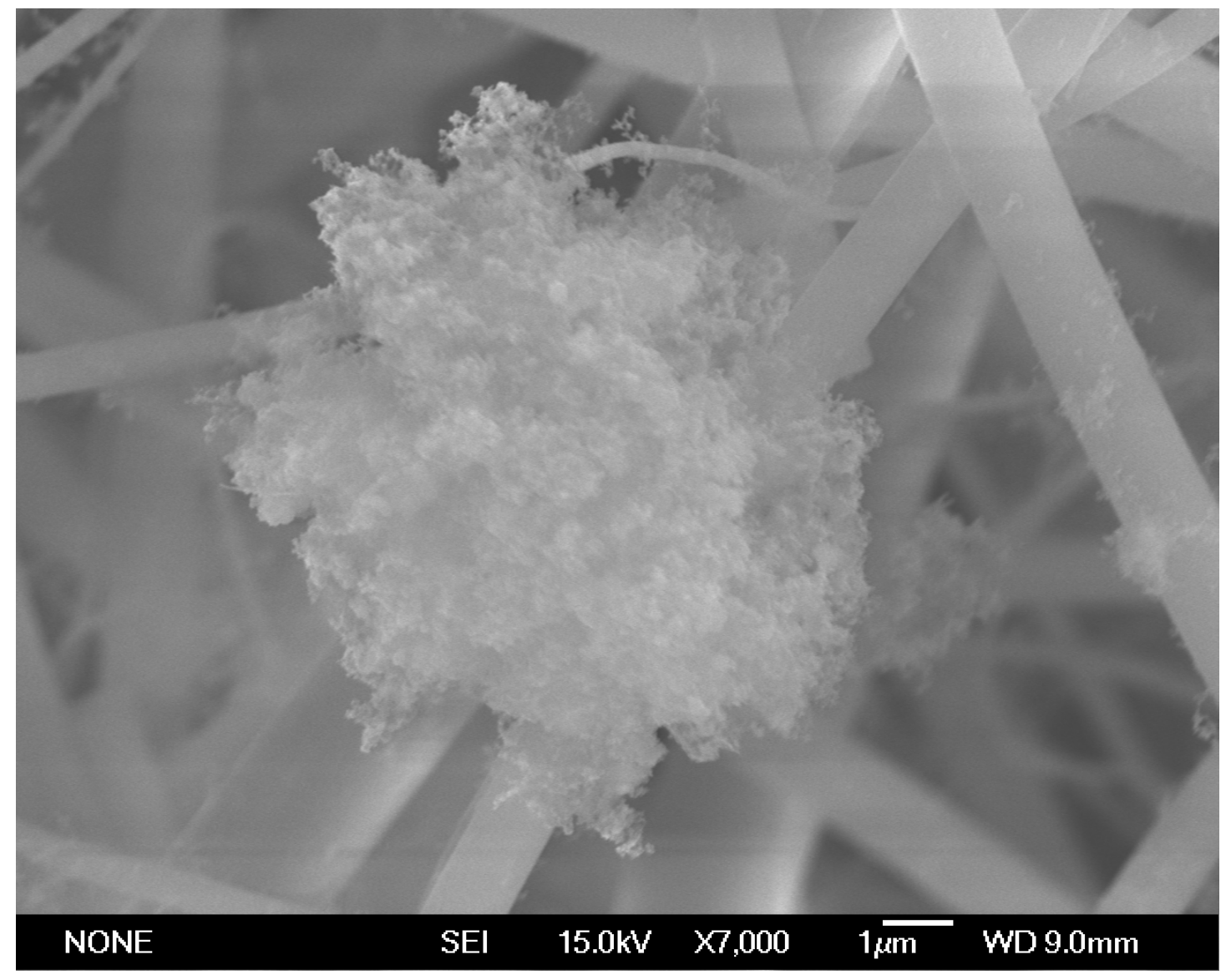

Figure 8.3 Representative SEM image of a carbonaceous particle agglomerate

\subsection{Conclusions}

This study aimed to characterize carbonaceous aerosols in the Mount Victoria Tunnel, an urban tunnel located in Wellington, New Zealand. The average $\mathrm{PM}_{2.5}$ concentration in the tunnel was $67.6 \pm 19.4 \mu \mathrm{g} \mathrm{m}^{-3}$, with between 63 and $73 \%$ of the PM collected made up of carbonaceous species. Organic carbon was found to be the primary constituent of the tunnel PM, contributing between 32 and $46 \%$ to total particulate matter mass. OC and EC was determined using both the NIOSH and IMPROVE thermal/optical protocols, with OC concentrations of $30.9 \mu \mathrm{g} \mathrm{m}^{-3}$ and 21.7 $\mu \mathrm{g} \mathrm{m}^{-3}$ for NIOSH and IMPROVE respectively, and EC concentrations of $18.6 \mu \mathrm{g} \mathrm{m}^{-3}$ and $21.3 \mu \mathrm{g} \mathrm{m}^{-3}$ for NIOSH and IMPROVE respectively. Total carbon concentrations determined by NIOSH and IMPROVE did not differ significantly, demonstrating that both techniques provide an adequate measure of total carbon in a sample. Most 
notably, it was found that total carbon determined using EA/IRMS directly correlated with TC determined using NIOSH, providing the first example of this correlation in the literature. In this study, when TC from EA/IRMS is combined with BC data determined using light reflection it was possible to obtain a measure of the OC present in a sample, providing an alternative analysis to thermal/optical methods for OC and EC determination in PM samples. Further research is required to validate this method for all aerosol types.

Stable isotope analysis of PM from the tunnel revealed $\delta^{13} \mathrm{C}$ values ranging from -24.7 to $-28.3 \%$, within the expected range for motor vehicles. The total average particle-phase PAH concentration in the tunnel was determined to be $70 \mathrm{ng}$ $\mathrm{m}^{-3}$, with low molecular weight PAHs ( $\leq 4$ rings) enriched relative to higher molecular weight PAHs. This result suggests that diesel vehicles contribute higher concentrations of PAHs compared to gasoline vehicles. The $\mathrm{I}[\mathrm{cd}] \mathrm{P} /(\mathrm{I}[\mathrm{cd}] \mathrm{P}+\mathrm{B}$ [ghi]P ratio was determined to be 0.32 , also suggesting that $\mathrm{PAH}$ emissions from diesel vehicles are the dominant source of PAHs in the tunnel.

SEM analysis of PM collected in the tunnel revealed that carbonaceous species made up the majority of particles collected in the tunnel. These species exhibited an amorphous morphology and formed large aggregates made up of 30 $100 \mathrm{~nm}$ spherules. Inorganic elements like $\mathrm{Fe}, \mathrm{O}, \mathrm{Na}, \mathrm{S}, \mathrm{Al}$ and $\mathrm{Zn}$ were found interspersed amongst the carbonaceous material, with their presence due to nonexhaust processes like brake and tire wear, and road dust re-suspension.

\subsection{Acknowledgements}

The authors thank David Flynn for providing support with the electron microscopy, Troy Baisden for use of the GC/MS and Philip Hopke for the OC/EC analysis. This work was partially funded by the New Zealand Foundation for Research, Science and Technology under contract C05X0903. 


\section{Masterton: Identification of particulate matter sources on an hourly time-scale in a wood burning community}

\subsection{Synopsis}

This chapter has been reproduced in full, with permission, as it appears in Environmental Science and Technology, 2012. Ancelet, T., Davy, P. K., Mitchell, T., Trompetter, W. J., Markwitz, A. and Weatherburn, D. C., 2012. Identification of particulate matter sources on an hourly time-scale in a wood burning community. Environmental Science and Technology, 46, 4767-4774.

In this study, PM sources at two different sites (Wairarapa College and Chanel College) in Masterton, New Zealand were investigated on an hourly time-scale. Streaker samplers were used to collect hourly, size-segregated $\mathrm{PM}_{10-2.5}$ and $\mathrm{PM}_{2.5}$ samples that were analyzed for elemental content using ion beam analysis techniques. Black carbon concentrations were determined using light reflection and $\mathrm{PM}_{10}$ concentrations were recorded using co-located continuous PM monitors. Prior to this study, the use of E-BAMs alongside Streaker samplers had never been reported.

$\mathrm{PM}_{10}$ concentrations at both sites displayed a diurnal pattern, with hourly $\mathrm{PM}_{10}$ concentration maxima in the evening (7 pm-midnight) and in the morning (7-9 am). The Chanel College site experienced consistently higher average $\mathrm{PM}_{10}$ concentrations during every hour and analysis indicated that katabatic flows across the urban area contributed to the increased concentrations observed. Source apportionment using positive matrix factorization on the hourly data revealed four primary $\mathrm{PM}_{10}$ sources for each site: biomass burning, motor vehicles, marine aerosol and crustal matter. Biomass burning was found to be the most dominant source at both sites and was responsible for both the evening and morning $\mathrm{PM}_{10}$ concentration peaks. While it was not surprising that biomass burning dominated the evening peak, since many Masterton residents use wood burners to heat their homes, it was surprising that biomass burning dominated the morning peak as well. This suggested that Masterton residents were re-lighting their fires when they rose in the morning. The use of elemental speciation combined with $\mathrm{PM}_{10}$ concentrations for source apportionment on an hourly time-scale had never been reported prior to this study, but it is clear that this 
type of analysis provides unique and useful information on PM sources for air quality management.

\subsection{Introduction}

Particulate matter (PM) is well-known to have adverse effects on human health (Dockery et al. 1993; Nel 2005; Russell and Brunekreef 2009). It also has a range of environmental effects, including local reductions in visibility and effects on the radiative balance (Tsai and Cheng 1999; Pósfai and Buseck 2010). Because of these effects, PM concentrations are routinely monitored in numerous countries and managed according to local legislation. In New Zealand, the National Environmental Standard (NES) for PM sets a 24-hour average limit for $\mathrm{PM}_{10}$ (particulate matter with aerodynamic diameters less than $10 \mu \mathrm{m}$ ) concentrations at $50 \mu \mathrm{g} \mathrm{m}^{-3}$. Many urban areas in New Zealand exceed the NES numerous times each year, particularly during the winter when wood combustion for home heating is common (http://www.mfe.govt.nz/environmental-reporting/report-cards/air/2009/index.html).

Particulate matter concentrations in New Zealand urban environments have been shown to have distinct diurnal cycles, independent of community size or population (Trompetter et al. 2010), with peak $\mathrm{PM}_{10}$ concentrations occurring between $10 \mathrm{pm}-$ midnight and 8-11 am. Critically, these peaks can push daily $\mathrm{PM}_{10}$ concentrations above the NES. Currently nothing is known about PM sources or their contributions on an hourly time-scale, information that is critical for the effective management of air quality. In an effort to identify the sources and factors contributing to PM pollution episodes on an hourly time-scale, an intensive monitoring campaign was undertaken in Masterton, New Zealand during the winter 2010. Masterton is a rural town located on a flat river plain with a population of approximately 20000 . During the winter, when wood combustion for home heating is common, Masterton exceeds the NES several times, primarily because of temperature inversion conditions limiting the dispersion of pollutants.

In this study, hourly coarse ( $\left.\mathrm{PM}_{10-2.5}\right)$ and fine $\left(\mathrm{PM}_{2.5}\right)$ samples were collected from two sites in Masterton (latitude $-40.9597^{\circ}$, longitude $175.658^{\circ}$ ) and analyzed for elemental content and black carbon (BC). Hourly $\mathrm{PM}_{10}$ concentrations were determined using continuous $\mathrm{PM}_{10}$ (BAM) monitors. Positive matrix factorization (PMF) was used to determine the PM sources and their contributions on an hourly 
time-scale at each site using the hourly elemental, $\mathrm{BC}$ and $\mathrm{PM}_{10}$ data. PMF is a powerful and commonly used multivariate method capable of resolving factors contributing to PM samples without prior source knowledge. PMF has a number of advantages over traditional factor analysis techniques including non-negativity constraints and the ability to accommodate missing or below detection limit data. The results of the analysis are directly interpretable as mass contributions from each factor (source) (Paatero and Tapper 1994; Paatero 1997; Song et al. 2001). Two receptor models are available to perform PMF, PMF2 (Paatero 1997) and EPA PMF (USEPA 2008). EPA PMF adopts a bilinear model which is solved by the Multilinear Engine (Paatero 1999) and incorporates a graphical user interface. When compared with PMF2, EPA PMF has been shown to provide similar results with some minor differences in the final solutions (Kim and Hopke 2007).

$\mathrm{PM}_{10}$ concentrations and PM sources and their contributions between the two sites were compared to identify potential PM transport mechanisms within Masterton. While the elemental characterization of hourly samples has been reported (Prati et al. 2000; D'Alessandro et al. 2003; Mazzei et al. 2007), to the best of our knowledge, no studies have applied PMF to hourly elemental data. PMF has previously been applied to 3-hourly size-resolved samples (Peré-Trepat et al. 2007). The application of PMF to hourly samples provides important information on source contributions at a temporal resolution capable of identifying not only the main PM sources at a particular time, but also more intermittent sources that may have too short a duration to be observed at a lower temporal resolution, for example in 24-hour integrated samples.

\subsection{Experimental Section}

\subsubsection{Sample Collection}

Ambient air monitoring was conducted on the grounds of two secondary schools in Masterton, Wairarapa College (WC) (latitude $-40.949945^{\circ}$, longitude $175.646574^{\circ}$ ) and Chanel College (CC) (latitude $-40.959873^{\circ}$, longitude $175.654693^{\circ}$ ). Figure 9.1 presents the locations of the two sampling sites. The CC site was approximately $120 \mathrm{~m}$ from the nearest road and $1 \mathrm{~km}$ from the central business

district and was situated along the general katabatic flow path (Manins and Sawford 
1979), approximately $1.2 \mathrm{~km}$ southeast of the WC site. The WC site was co-located with an ambient air quality monitoring station used for compliance monitoring and operated by the Greater Wellington Regional Council (site established October 2002). The site was $150 \mathrm{~m}$ from the nearest road and approximately $1 \mathrm{~km}$ from the central business district. The land around both sites was flat and surrounded by open space or school and residential buildings no more than two stories high.

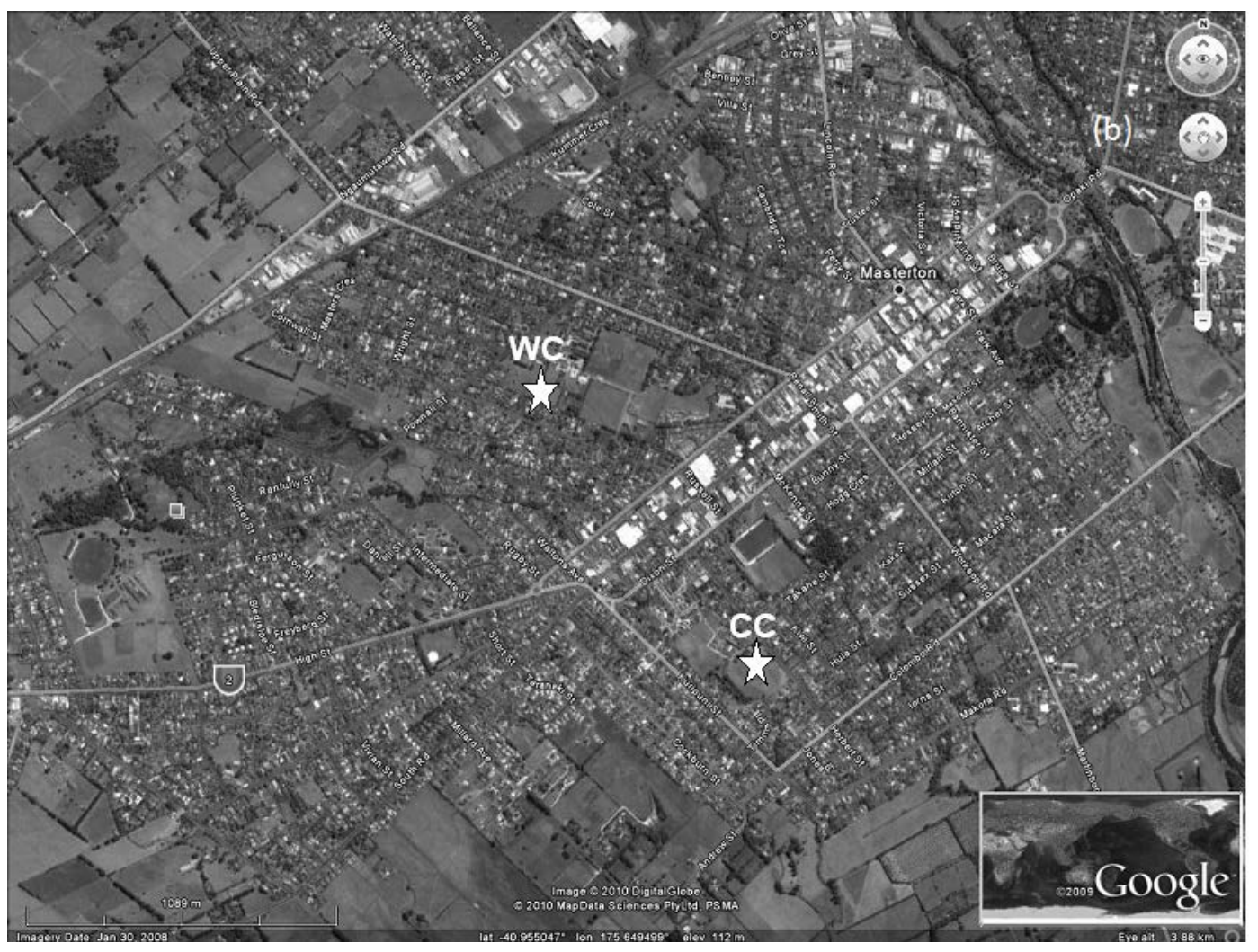

Figure 9.1 Locations of the sampling sites within Masterton (WC-Wairarapa College; CC-

Chanel College).

Size-segregated coarse and fine PM samples at each site were collected using Streaker samplers (PIXE International Corporation, USA). The Streaker sampler has previously been described in detail (Annegarn et al. 1988), and used in a number of studies (Annegarn et al. 1992; Annegarn et al. 1996; Filippi et al. 1999; D'Alessandro et al. 2004). Briefly, the Streaker consists of a pre-impactor that removes particles larger than $\mathrm{PM}_{10}$ from the incoming air flow, a thin Kapton foil that collects coarse particles through impaction and a Nucleopore film that collects fine particles. Hourly samples were collected with discrete spacing between each deposit to ensure that each deposit only consisted of PM collected during the intended hour. An image of a typical fine Streaker filter after sampling is presented in Figure 9.2. A total of 60 
samples, or 60 hours, were collected on each set of filters. The monitoring program ran from July-September 2010 and a total of 1251 samples were collected between the two sites (571 from WC and 680 from CC).

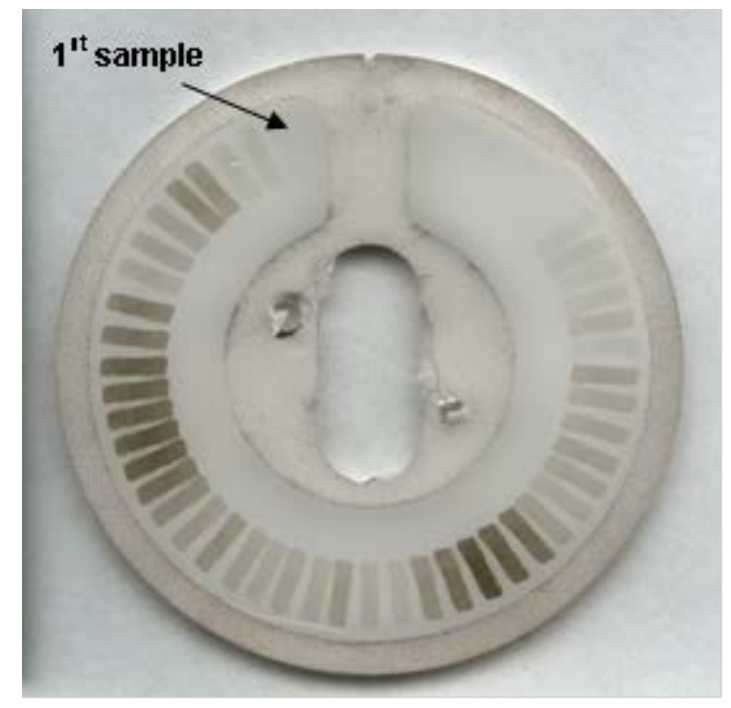

Figure 9.2 Streaker sampler filter showing individual hourly samples (dark bands)

Because the 60 discrete hourly PM samples are collected on a single Streaker filter, gravimetric determination of the hourly PM mass was not possible. Therefore a continuous E-BAM (Met One Instruments, Inc.) was co-located with each Streaker sampler to obtain hourly $\mathrm{PM}_{10}$ concentrations. E-BAMs calculate particle mass based on beta-particle attenuation through a filter tape. The co-location of Streaker samplers with E-BAMs has never been reported before. Sonic anemometers (Vaisala WXT520) at heights of $10 \mathrm{~m}$, were used at both sites to obtain meteorological parameters such as wind speed and direction, temperature and relative humidity.

\subsubsection{Elemental Analysis}

Ion beam analysis (IBA) was used to measure the concentrations of elements with atomic numbers above neon in the PM collected. IBA measurements were carried out at the New Zealand Ion Beam Analysis Facility operated by the Institute of Geological and Nuclear Sciences (GNS) in Gracefield, Lower Hutt, New Zealand (Trompetter et al. 2005). The full suite of analyses included particle-induced X-ray emission (PIXE), particle-induced gamma-ray emission (PIGE) and Rutherford backscattering (RBS). Black carbon was measured using a M43D Digital Smoke Stain Reflectometer. The determination of BC concentrations from the reflectometer 
measurements has been reported previously (Ancelet et al. 2011b). The IBA process provides analytical uncertainties and limits of detection (LODs) for each element in each of the discrete hourly PM samples, with variations in these values dependent on the experimental conditions, filter matrix and sample loading. Prior to the PMF analyses, data and uncertainty matrices were prepared for each site in the same manner as previous studies (Polissar et al. 1998; Song et al. 2001).

\subsubsection{Receptor Modeling}

Receptor modeling and apportionment of PM mass by PMF was performed using the EPAPMF version 3.0.2.2 program in accordance with the User's Guide (USEPA 2008). With PMF, sources are constrained to have non-negative species concentrations, no sample can have a negative source contribution and error estimates for each observed point are used as point-by-point weights. This is a distinct advantage of PMF, since it can accommodate missing or below detection limit data that is a common feature of environmental monitoring (Song et al. 2001). Since the signal-to-noise ratio $(\mathrm{S} / \mathrm{N})$ for an individual element can have a significant influence on a receptor model, it has been strongly suggested to down-weight or discard noisy variables (Paatero and Hopke 2003). Therefore, the data in this study were screened by their $\mathrm{S} / \mathrm{N}$. Variables with very low $\mathrm{S} / \mathrm{N}(\leq 0.2)$ were excluded from the analysis, while weak variables $(0.2 \leq \mathrm{S} / \mathrm{N} \leq 2.0)$ were down-weighted. Rotational freedom in solutions was explored and controlled using FPEAK (Paatero et al. 2002) and observing the effect on the $\mathrm{Q}$ values (chi squared), G-vector and residual plots (Paatero et al. 2005). Solutions were also assessed for consistency across multiple runs by comparing $Q$ values and residual analysis ( $D$ values) to ensure that a true global minimum had been achieved (USEPA 2008). Only those solutions that could be related to physical sources were considered acceptable. PMF was applied to the coarse and fine elemental data together to obtain the $\mathrm{PM}_{10}$ sources and their contributions. Table 9.1 provides a summary of the parameters used for the PMF analyses and the diagnostics obtained for the WC and CC sites. 
Table 9.1 Parameters used for PMF analyses and diagnostics obtained from the analyses for Wairarapa College and Chanel College

\begin{tabular}{|l|l|l|}
\hline & Wairarapa College & Chanel College \\
\hline $\begin{array}{l}\text { Extra modeling } \\
\text { uncertainty (\%) }\end{array}$ & 15 & 15 \\
\hline Number of base runs & 20 & 20 \\
\hline Base run seed & Random & Random \\
\hline Number of bootstraps & 200 & 200 \\
\hline $\begin{array}{l}\text { Minimum correlation R- } \\
\text { value }\end{array}$ & 0.6 & 0.6 \\
\hline Bootstrap seed & Random & Random \\
\hline Block size & 24 & 24 \\
\hline Theoretical Q & 7613 & 8806 \\
\hline Robust Q & 6097 & 5900 \\
\hline True Q & 6098 & 5901 \\
\hline $\begin{array}{l}\text { Number of bootstrapped } \\
\text { factors mapped to original } \\
\text { factor 1 }\end{array}$ & 200 & 200 \\
\hline $\begin{array}{l}\text { Number of bootstrapped } \\
\text { factors mapped to original } \\
\text { factor 2 }\end{array}$ & 200 & 200 \\
\hline $\begin{array}{l}\text { Number of bootstrapped } \\
\text { factors mapped to original } \\
\text { factor 3 }\end{array}$ & 200 & 197 \\
\hline $\begin{array}{l}\text { Number of bootstrapped } \\
\text { factors mapped to original } \\
\text { factor 4 }\end{array}$ & 199 & 0.6 \\
\hline $\begin{array}{l}\text { Number of bootstrapped } \\
\text { factors mapped to no } \\
\text { original factor }\end{array}$ & 1 & 200 \\
\hline Maximum D value & 0.4 & \\
\hline
\end{tabular}

\subsection{Results and Discussion}

\subsection{1 $P M_{10}$ Concentrations}

$\mathrm{PM}_{10}$ concentrations at the two sites displayed distinct diurnal cycles. Figure 9.3 presents the average hourly $\mathrm{PM}_{10}$ concentrations at the WC and CC sites over the entire sampling period. Two distinct features are apparent in Figure 9.3. First, the diurnal cycles at the two sites were similar, with peak $\mathrm{PM}_{10}$ concentrations occurring between 7 pm-midnight and 7-9 am. Secondly, average hourly $\mathrm{PM}_{10}$ concentrations at CC were higher than those at WC. The morning peak, in particular, was significantly higher at CC. Comparison of daily hourly average $\mathrm{PM}_{10}$ concentrations between the two sites revealed that WC exceeded the New Zealand NES for $\mathrm{PM}_{10}$ of 
$50 \mu \mathrm{g} \mathrm{m}^{-3}$ six times during the sampling period, while the CC site experienced 18 exceedances of the NES. It should be noted that E-BAMs are not certified for air quality compliance monitoring, but the results of this analysis do indicate that $\mathrm{PM}_{10}$ concentrations within Masterton differ depending on the sampling location.

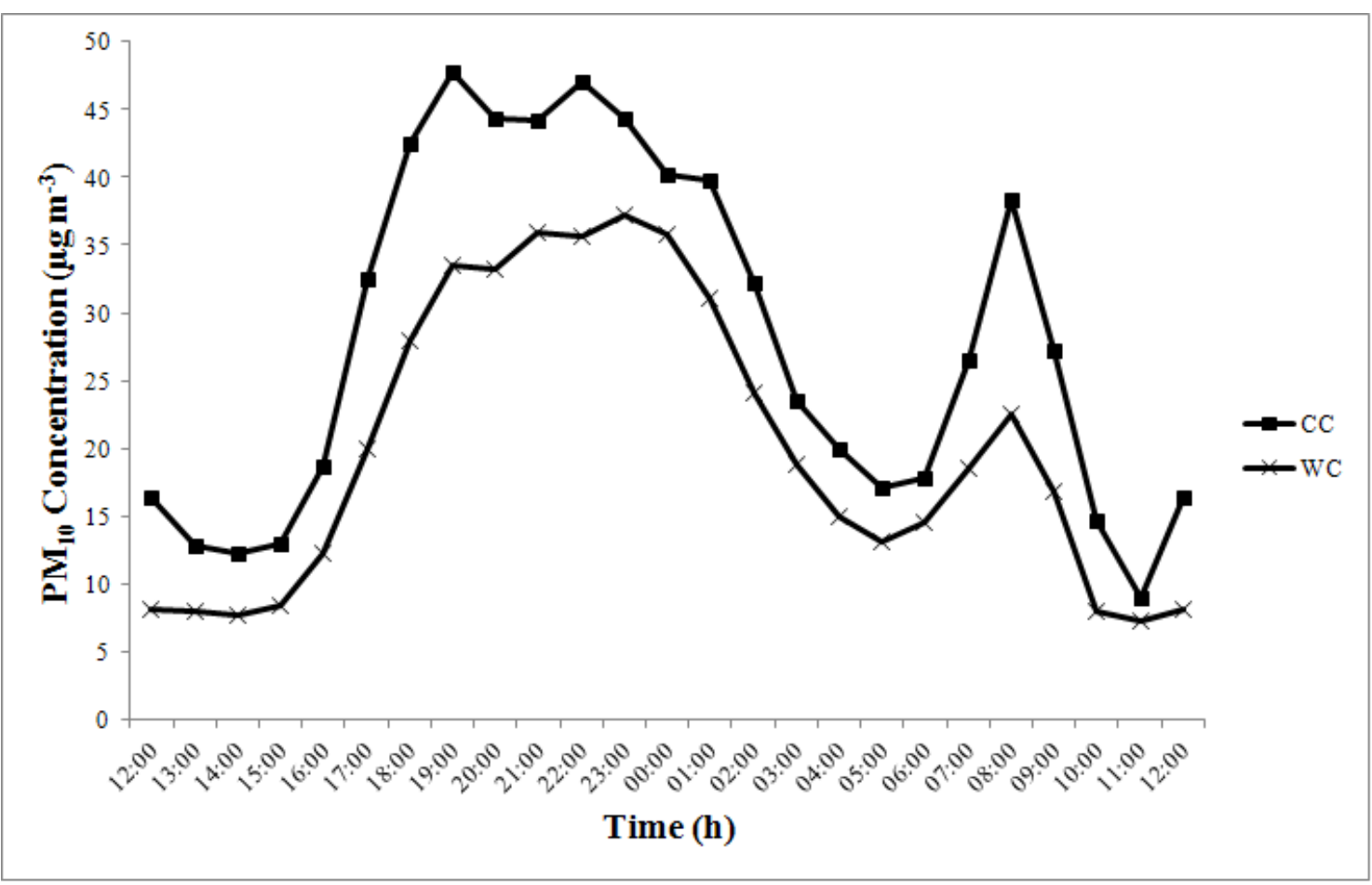

Figure 9.3 Average hourly $\mathrm{PM}_{10}$ concentrations at the Wairarapa College and Chanel College sites from July-September 2010.

To gain a better understanding of PM transport within Masterton, the local meteorology was investigated on an hourly basis. Figure 9.4 presents a wind rose plot over the entire sampling period. Winds during the sampling period were predominantly from the southwest, with smaller proportions from the north and northeast. With the high temporal resolution data available in this study, hourly pollution roses for the CC site were developed using the R statistical and Openair packages (Carslaw and Ropkins 2011; R Development Core Team 2011) and are presented in Figure 9.5. The hourly pollution roses indicated that $\mathrm{PM}_{10}$ measured at the CC site during the evening and morning was largely transported from the north, suggesting that the increased $\mathrm{PM}_{10}$ concentrations measured at $\mathrm{CC}$ were the result of katabatic flows southeastward following the downslope contour of the river plain. A similar katabatic flow mechanism has been observed in Christchurch, New Zealand (McKendry et al. 2004). 


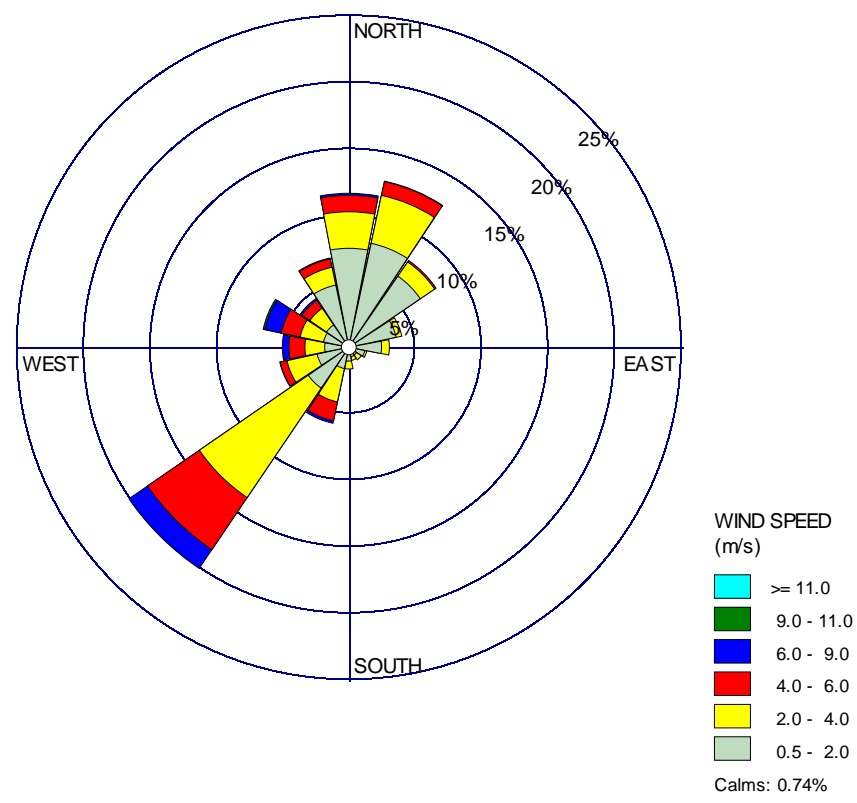

Figure 9.4 Wind rose plot over the entire sampling period from the Wairarapa College site. The radial dimensions indicate the frequency (\%) of winds from each direction. 


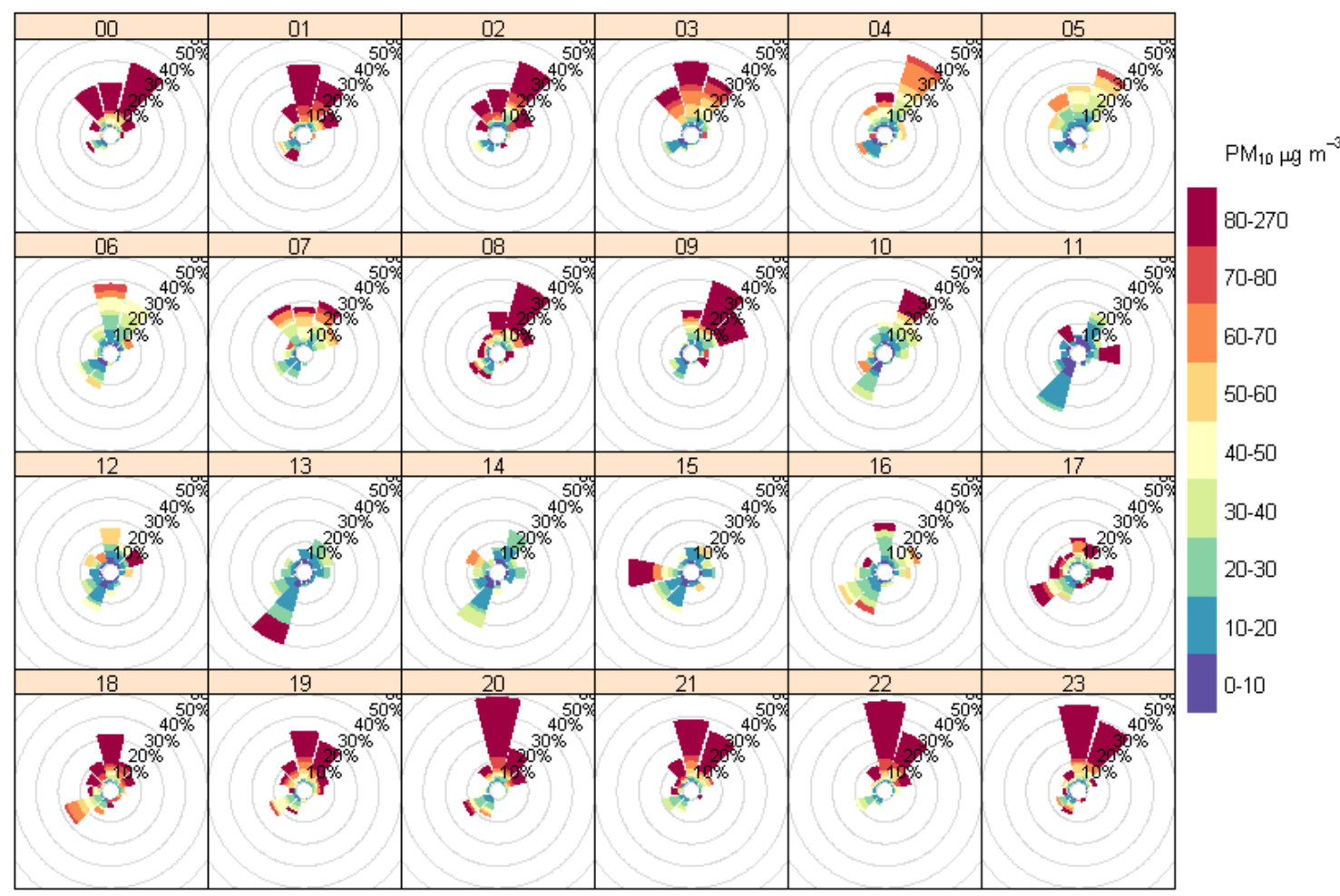

Proportion contribution to the mean (\%)

Figure 9.5 Hourly pollution roses from the Chanel College site (produced using the Openair package (Carlslaw and Ropkins, 2011)) indicating wind directions contributing the most to average hourly $\mathrm{PM}_{10}$ concentrations. The radial dimensions indicate the percentage of the total pollution that arrives from each wind sector during each one-hour period.

\subsubsection{Sources of Ambient $P M_{10}$}

Tables 9.2 and 9.3 present the average, maximum, minimum and median hourly concentrations of species used for source apportionment at the WC and CC sites, respectively, along with standard deviations, average uncertainties, average limits of detection and the number of samples above the limit of detection for each species. 


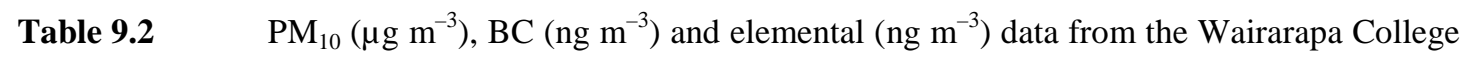
site

\begin{tabular}{|c|c|c|c|c|c|c|c|c|}
\hline & Average & Maximum & Minimum & Median & $\begin{array}{c}\text { Standard } \\
\text { Deviation }\end{array}$ & $\begin{array}{c}\text { Average } \\
\text { Uncertainty }\end{array}$ & $\begin{array}{c}\text { Average } \\
\text { LOD }\end{array}$ & $\begin{array}{c}\text { Number } \\
\text { LOD }\end{array}$ \\
\hline $\mathrm{PM}_{10}$ & 25 & 213 & 0 & 14 & 30 & & & \\
\hline $\begin{array}{c}\text { Fine } \\
\mathrm{BC}\end{array}$ & 3144 & 21652 & 157 & 1566 & 3797 & 611 & 800 & 552 \\
\hline Fine S & 234 & 1549 & 97 & 210 & 129 & 32 & 28 & 571 \\
\hline Fine K & 231 & 2036 & 0 & 117 & 273 & 113 & 27 & 565 \\
\hline $\begin{array}{c}\text { Fine } \\
\text { Ca }\end{array}$ & 104 & 770 & 0 & 81 & 91 & 109 & 24 & 565 \\
\hline $\begin{array}{c}\text { Fine } \\
\text { Fe }\end{array}$ & 38 & 376 & 0 & 29 & 35 & 115 & 9 & 553 \\
\hline $\begin{array}{c}\text { Fine } \\
\text { As }\end{array}$ & 7 & 91 & 0 & 0 & 15 & 108 & 35 & 41 \\
\hline $\begin{array}{c}\text { Coarse } \\
\text { Na }\end{array}$ & 1544 & 57044 & 0 & 718 & 2779 & 283 & 567 & 466 \\
\hline $\begin{array}{c}\text { Coarse } \\
\text { Al }\end{array}$ & 375 & 17901 & 0 & 71 & 1578 & 340 & 77 & 374 \\
\hline $\begin{array}{c}\text { Coarse } \\
\text { Si }\end{array}$ & 475 & 9453 & 121 & 302 & 638 & 31 & 51 & 571 \\
\hline $\begin{array}{c}\text { Coarse } \\
\text { S }\end{array}$ & 197 & 4767 & 0 & 101 & 303 & 150 & 39 & 520 \\
\hline $\begin{array}{c}\text { Coarse } \\
\text { Cl }\end{array}$ & 1555 & 51507 & 0 & 386 & 2914 & 119 & 36 & 571 \\
\hline $\begin{array}{c}\text { Coarse } \\
\text { K }\end{array}$ & 134 & 3099 & 0 & 83 & 179 & 157 & 37 & 532 \\
\hline $\begin{array}{c}\text { Coarse } \\
\text { Ca }\end{array}$ & 764 & 25749 & 114 & 351 & 2468 & 25 & 32 & 512 \\
\hline $\begin{array}{c}\text { Coarse } \\
\text { Fe }\end{array}$ & 137 & 7428 & 0 & 52 & 383 & 78 & 18 & 539 \\
\hline
\end{tabular}




\begin{tabular}{|c|c|c|c|c|c|c|c|c|}
\hline & Average & Maximum & Minimum & Median & $\begin{array}{c}\text { Standard } \\
\text { Deviation }\end{array}$ & $\begin{array}{c}\text { Average } \\
\text { Uncertainty }\end{array}$ & $\begin{array}{c}\text { Average } \\
\text { LOD }\end{array}$ & $\begin{array}{c}\text { Number } \\
\text { > LD }\end{array}$ \\
\hline PM $_{10}$ & 32 & 484 & 0 & 19 & 39 & & & \\
\hline $\begin{array}{c}\text { Fine } \\
\text { BC }\end{array}$ & 3716 & 20449 & 139 & 2011 & 4007 & 623 & 800 & 631 \\
\hline Fine S & 236 & 645 & 106 & 228 & 71 & 22 & 37 & 680 \\
\hline Fine K & 148 & 891 & 0 & 82 & 163 & 15 & 25 & 601 \\
\hline $\begin{array}{c}\text { Fine } \\
\text { Ca }\end{array}$ & 56 & 339 & 0 & 48 & 38 & 21 & 22 & 554 \\
\hline $\begin{array}{c}\text { Fine } \\
\text { Fe }\end{array}$ & 38 & 2774 & 0 & 22 & 152 & 11 & 8 & 554 \\
\hline $\begin{array}{c}\text { Fine } \\
\text { As }\end{array}$ & 6 & 110 & 0 & 0 & 14 & 31 & 34 & 38 \\
\hline $\begin{array}{c}\text { Coarse } \\
\text { Na }\end{array}$ & 508 & 13098 & 0 & 300 & 713 & 294 & 499 & 322 \\
\hline $\begin{array}{c}\text { Coarse } \\
\mathrm{Al}\end{array}$ & 110 & 2297 & 25 & 81 & 163 & 36 & 55 & 612 \\
\hline $\begin{array}{c}\text { Coarse } \\
\mathrm{Si}\end{array}$ & 168 & 2498 & 62 & 113 & 190 & 32 & 42 & 680 \\
\hline $\begin{array}{c}\text { Coarse } \\
\mathrm{S}\end{array}$ & 71 & 1510 & 0 & 49 & 89 & 34 & 51 & 410 \\
\hline $\begin{array}{c}\text { Coarse } \\
\mathrm{Cl}\end{array}$ & 643 & 17110 & 0 & 280 & 1046 & 57 & 41 & 672 \\
\hline $\begin{array}{c}\text { Coarse } \\
\mathrm{K}\end{array}$ & 42 & 1916 & 0 & 29 & 81 & 21 & 32 & 465 \\
\hline $\begin{array}{c}\text { Coarse } \\
\mathrm{Ca}\end{array}$ & 164 & 5565 & 40 & 106 & 387 & 27 & 28 & 680 \\
\hline $\begin{array}{c}\text { Coarse } \\
\text { Fe }\end{array}$ & 54 & 4172 & 0 & 14 & 232 & 14 & 13 & 498 \\
\hline
\end{tabular}

Application of PMF to the hourly elemental data revealed four primary $\mathrm{PM}_{10}$ sources at the WC and CC sites. The source profiles obtained for WC and CC are presented in Figures 9.6 and 9.7, respectively. The error bars in Figures 9.6 and 9.7 indicate standard deviations determined from bootstrapping in the EPAPMF program. The EPAPMF bootstrapping reanalysis (block size $=24$ ) of the dataset provides an indication of the variation around the original factor profile concentrations and a measure of the rotational ambiguity in the selected solution. Unfortunately, the bootstrapping technique is unable to fully account for the rotational ambiguity of the solution, but for the solutions presented in this manuscript we found that FPEAK rotations had little or no effect on the solution (factor profiles and time-series), indicating a stable and robust result.

Reconstructed masses (RCMs) obtained using the elemental data accounted for 22 and 27\% of the $\mathrm{PM}_{10}$ mass at the WC and CC sites, respectively (Malm et al. 1994). The relatively low RCMs for both sites were not surprising, since numerous species, including organic carbon (OC) were not quantified. For sources featuring little contribution from unmeasured species (marine aerosol and crustal matter), the RCMs were close to those identified from PMF. 

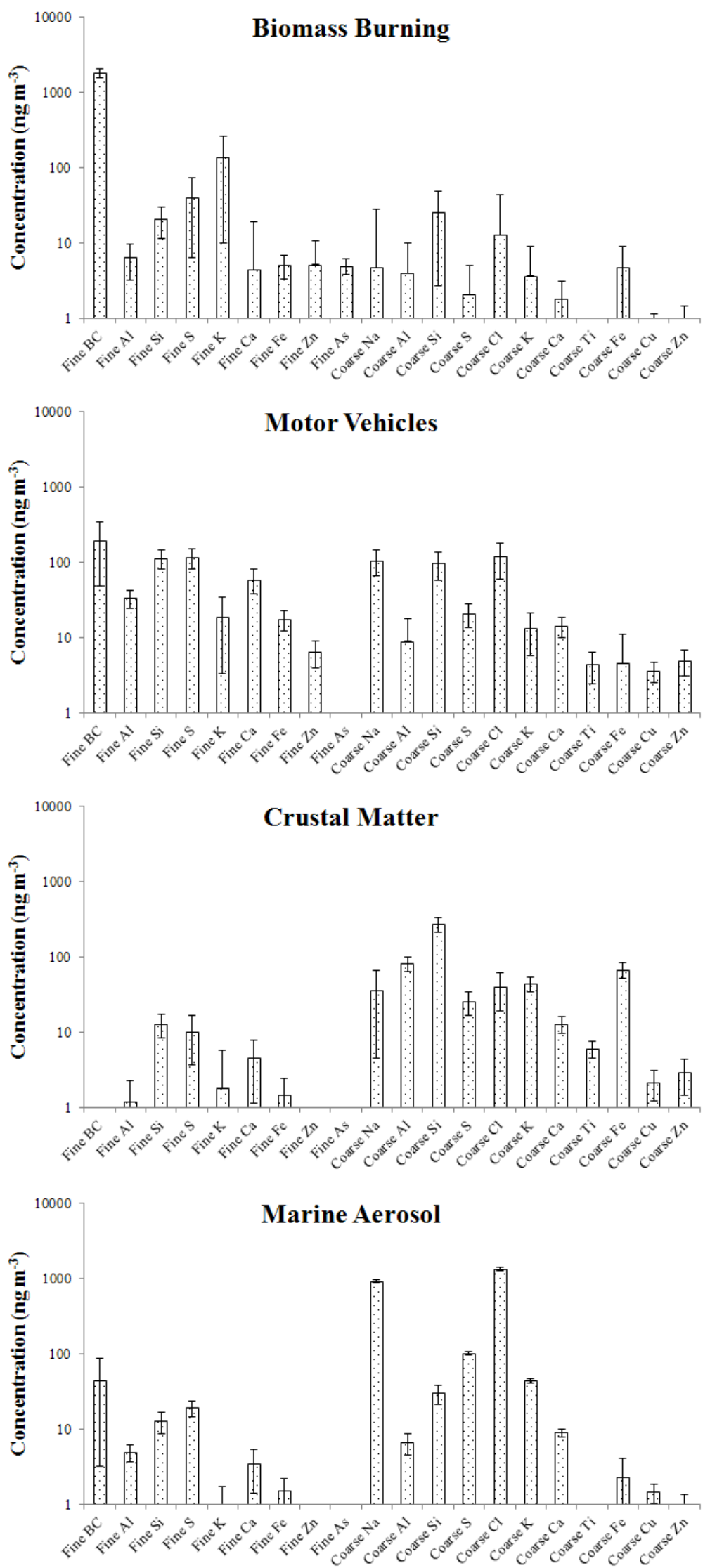

Figure 9.6 Source profiles obtained at Wairarapa College. 

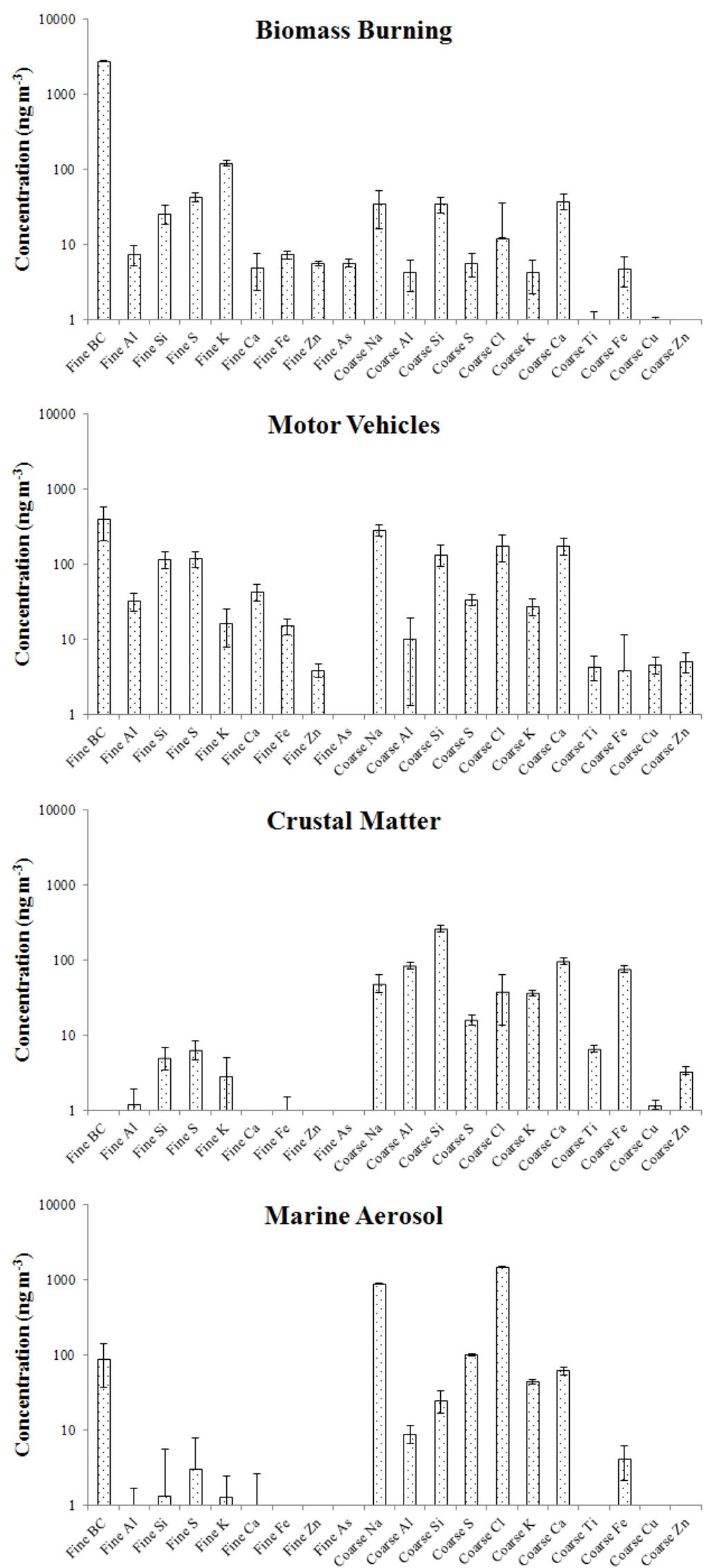

Figure 9.7 Source profiles obtained at Chanel College. 
The sources presented in Figures 9.6 and 9.7 were found to explain, on average, 89 and $90 \%$ of the $\mathrm{PM}_{10}$ mass measured by the E-BAMs at WC and CC, respectively, after regression using $\mathrm{PM}_{10}$ concentrations. Factor one made up 85 and $84 \%$ of the $\mathrm{PM}_{10}$ mass at the WC and CC sites, respectively. It was identified as a biomass burning source because of the high $\mathrm{BC}$ and fine $\mathrm{K}$ loadings. Potassium is typically used alongside BC as a marker for biomass burning, and wood combustion in particular (Fine et al. 2002; Khalil and Rasmussen 2003). Iron was also present in the biomass burning source profiles, likely as a result of the composition of home heating appliances (cast iron/steel) rather than the composition of the wood (Ancelet et al. 2011a). The biomass burning source profiles for each site were the only profiles that featured arsenic, indicating that at least some homes were using copper chrome arsenate (CCA)-treated timber as fuel for domestic heating purposes. CCA-treated timber is no longer commonly used in other parts of the world, but it is frequently used for construction purposes in New Zealand to prevent fungal and insect attacks. While the average arsenic concentration at both sites was $7 \mathrm{ng} \mathrm{m}^{-3}$, concentrations as high as 91 and $110 \mathrm{ng} \mathrm{m}^{-3}$ were observed at WC and CC, respectively. The use of CCA-treated timber was probably intermittent and opportunistic, since only a moderate correlation $\left(r^{2}=0.62\right)$ was observed between arsenic concentrations and the biomass burning source contributions on an hourly basis. To the best of our knowledge, the association of arsenic with biomass burning through receptor modeling has never been reported. We suggest that the higher concentrations observed at the CC site resulted from the greater number of emission sources (residential dwellings) upwind in the katabatic flow and this is supported by the discussion in Chapter 9.4.1 and by a plot of the biomass burning source contributions, for matching dates and times, from both sites (Figure 9.8). Figure 9.8 indicated that the biomass burning source contribution was $20 \%$ higher at the CC site. 


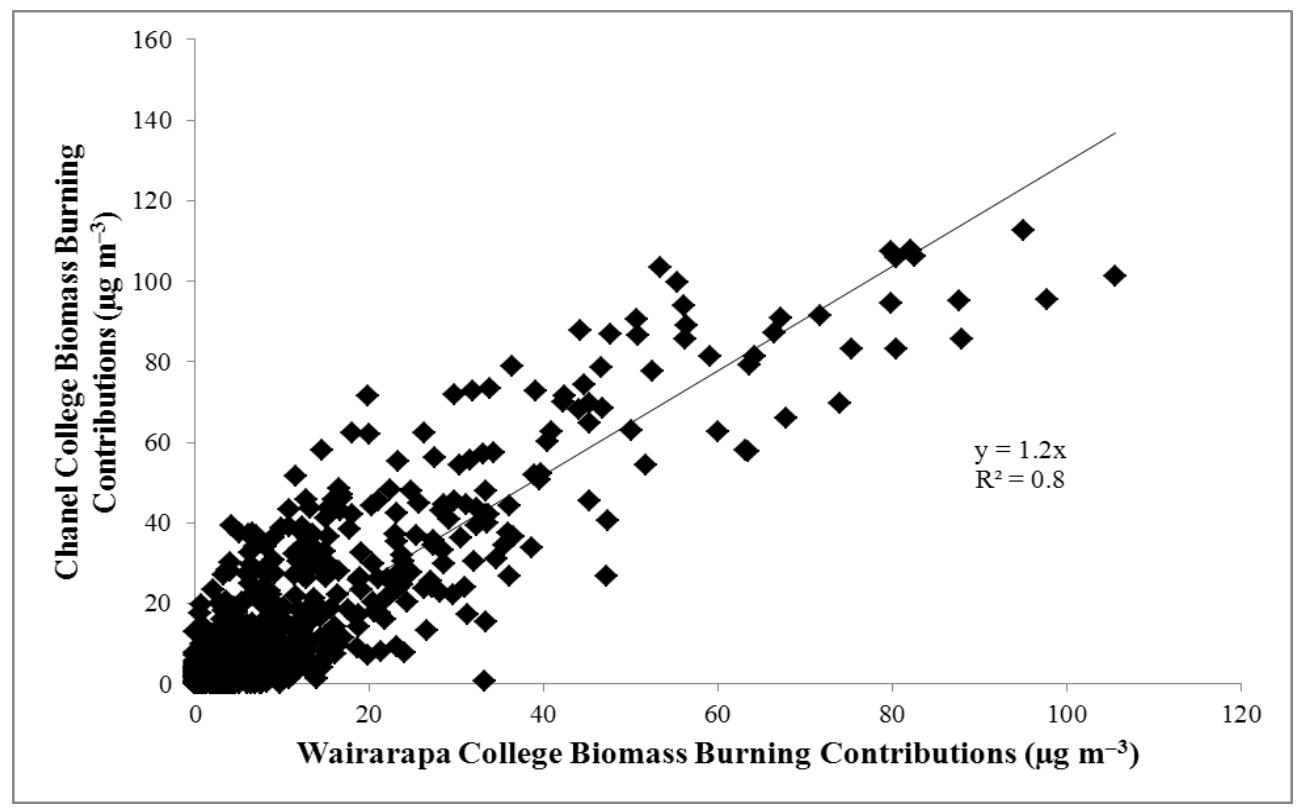

Figure 9.8 Plot of biomass burning contributions at Chanel College versus biomass burning contributions at Wairarapa College.

Factor two accounted for 10 and $13 \%$ of the $\mathrm{PM}_{10}$ mass at the WC and CC sites, respectively. This factor was characterized as a motor vehicle source, which included vehicular exhaust and non-exhaust emissions (road dust). Road dust is generated by the turbulent passage of motor vehicles over local roads and the source profile features crustal elements ( $\mathrm{Al}$ and $\mathrm{Si}$ ) enriched with $\mathrm{BC}, \mathrm{Ca}$ and Fe. Similar road dust source profiles have been reported (Garg et al. 2000; Schauer et al. 2006). Black carbon in the profile is associated with exhaust emissions, deposited tailpipe emissions and the abrasion of tar-sealed surfaces. Iron is typically present in brake wear dust (Thorpe and Harrison 2008).

The third factor was characterized as a soil, or crustal matter, source based on the presence of $\mathrm{Al}, \mathrm{Si}, \mathrm{K}, \mathrm{Ca}$ and Fe. The crustal matter factor contributed 2 and $0.6 \%$ of the $\mathrm{PM}_{10}$ mass at WC and CC, respectively. The fourth factor contributed 3 and 2.4 $\%$ of the $\mathrm{PM}_{10}$ mass and was marine aerosol because of high $\mathrm{Na}$ and $\mathrm{Cl}$ concentrations. Marine aerosol is a common $\mathrm{PM}_{10}$ source throughout the country due to New Zealand's remote oceanic location.

Hourly source contribution roses were prepared in a similar manner to the hourly pollution roses discussed in Section 9.4.1. Figure 9.9 presents the hourly biomass burning source roses for the CC site, while pollution roses for the motor vehicle, marine aerosol and crustal matter sources at the CC site are presented in 
Figures 9.10, 9.11 and 9.12, respectively. Similar source contribution roses were observed for the WC site. Figure 9.9 indicates that during the morning and evening large contributions from biomass burning resulted from air masses north of the site, providing further support for the katabatic flow mechanism suggested in Chapter 9.4.1. The motor vehicle source was less influenced by katabatic flows than the biomass burning source, while the marine aerosol and crustal matter sources were mainly influenced only by the prevailing wind directions. This result can be considered typical, since katabatic flows result in low wind speeds that are not conducive to elevated marine aerosol and crustal matter concentrations.

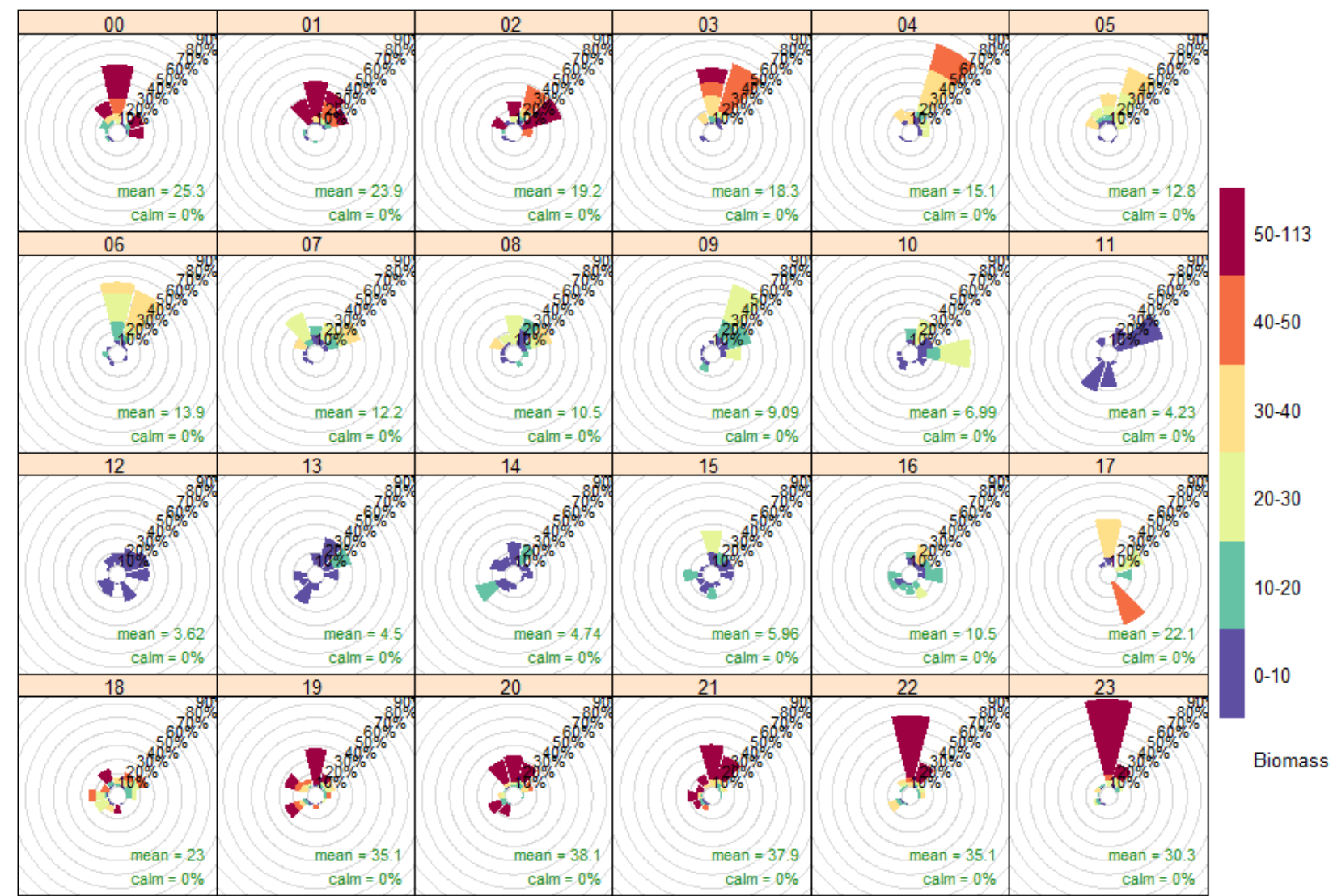

Proportion contribution to the mean (\%)

Figure 9.9 Hourly biomass burning source roses from the Chanel College site indicating wind directions contributing the most to average hourly $\mathrm{PM}_{10}$ concentrations. The radial dimensions indicate the percentage of the total biomass burning contribution that arrives from each wind sector during each one-hour period. 


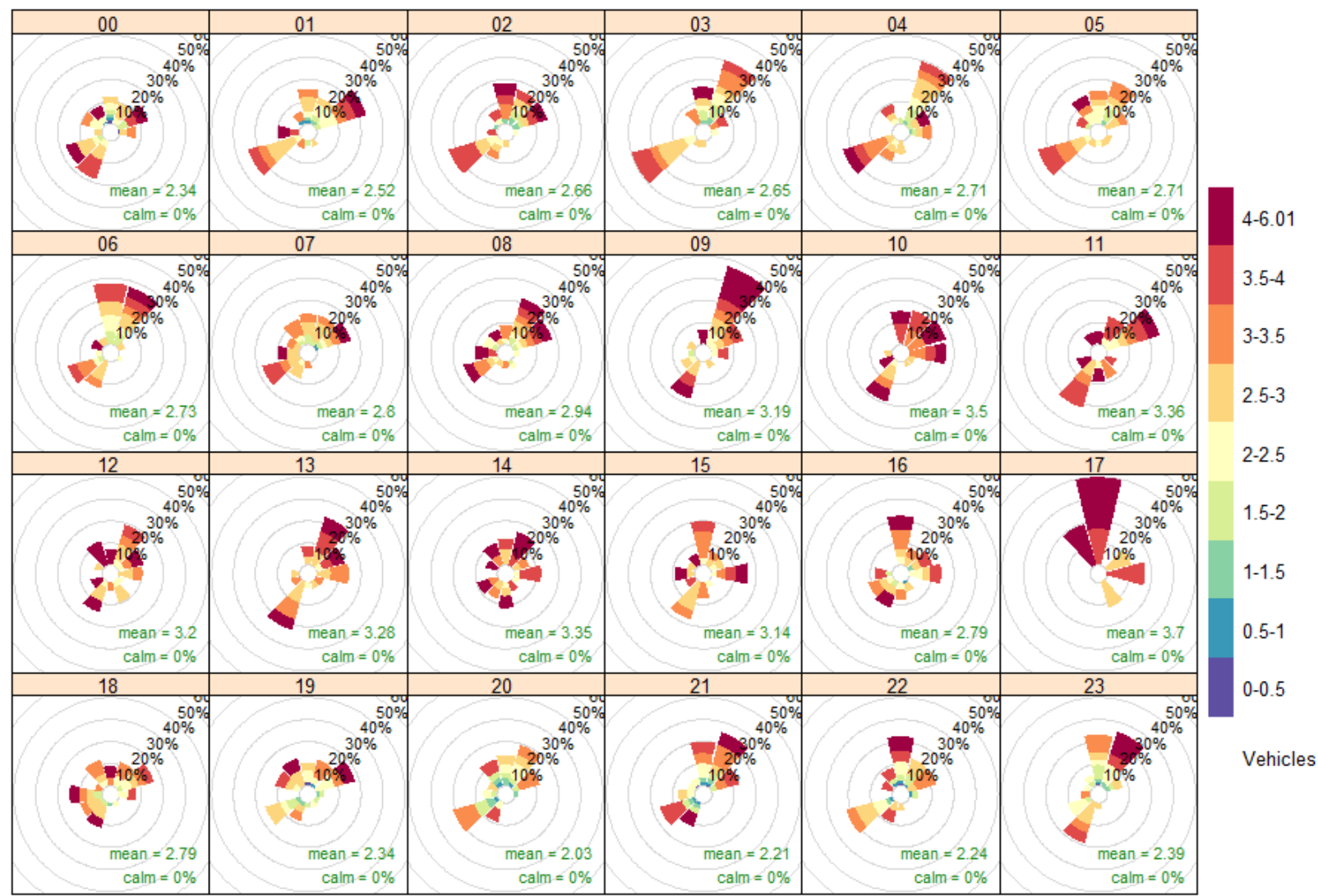

Proportion contribution to the mean (\%)

Figure 9.10 Hourly motor vehicle source roses from the Chanel College site indicating wind directions contributing the most to average hourly $\mathrm{PM}_{10}$ concentrations. The radial dimensions indicate the percentage of the total motor vehicle contribution that arrives from each wind sector during each one-hour period. 


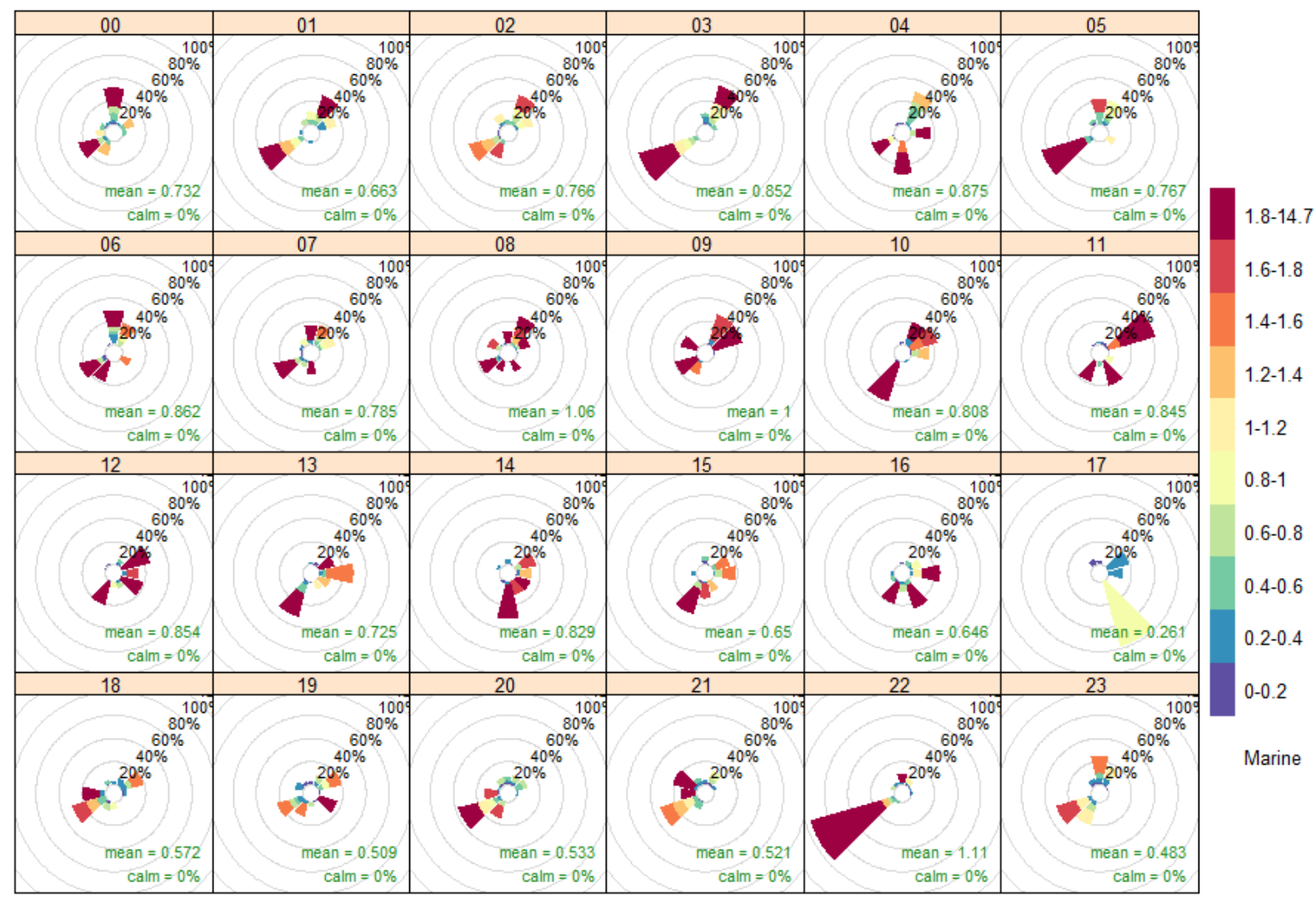

Proportion contribution to the mean (\%)

Figure 9.11 Hourly marine aerosol source roses from the Chanel College site indicating wind directions contributing the most to average hourly $\mathrm{PM}_{10}$ concentrations. The radial dimensions indicate the percentage of the total marine aerosol contribution that arrives from each wind sector during each one-hour period. 


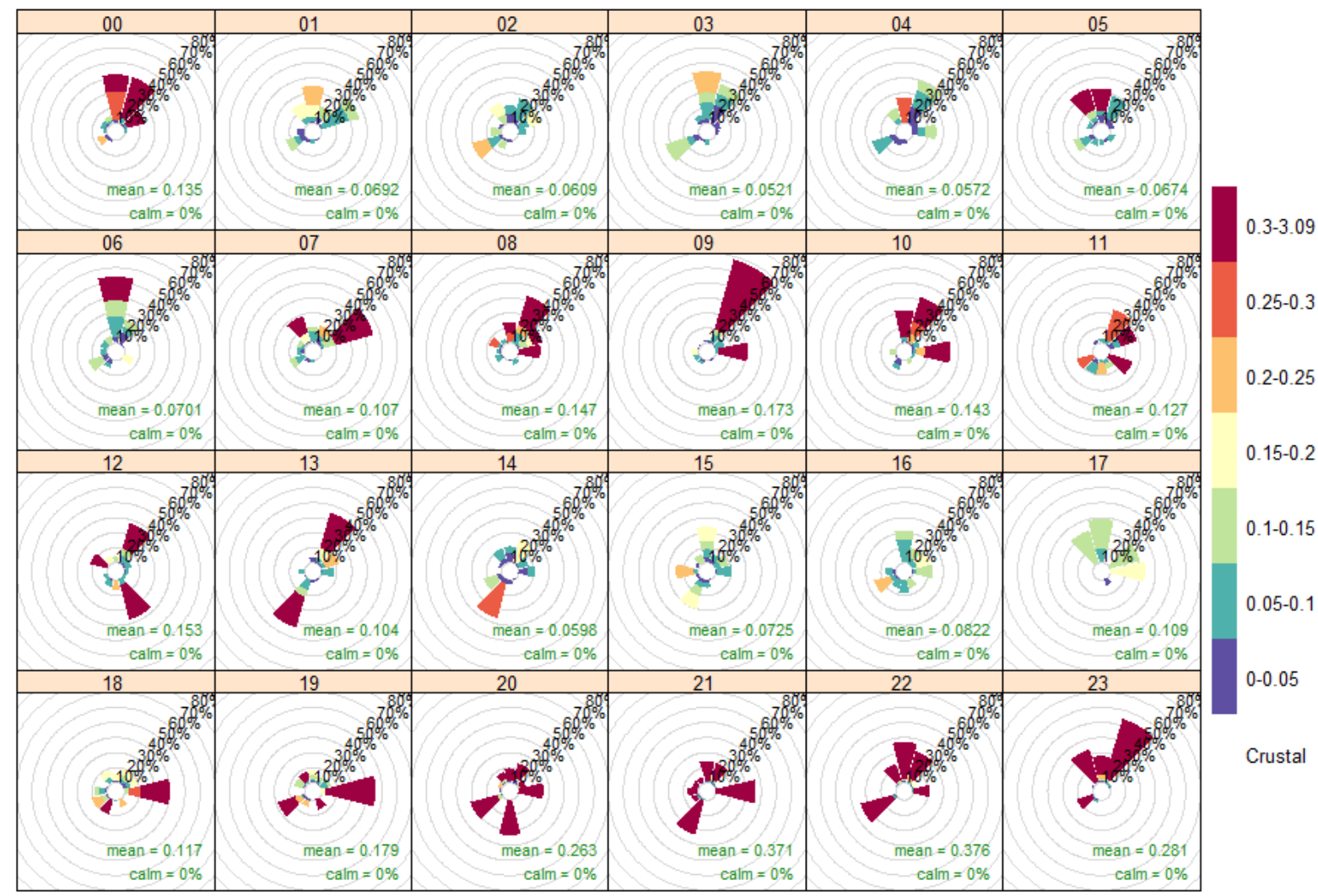

Proportion contribution to the mean (\%)

Figure 9.12 Hourly crustal matter source roses from the Chanel College site indicating wind directions contributing the most to average hourly $\mathrm{PM}_{10}$ concentrations. The radial dimensions indicate the percentage of the total crustal matter contribution that arrives from each wind sector during each one-hour period.

The average hourly contributions from each source at both sites were calculated to assess source contributions over every hour in a day. Figures 9.13 and 9.14 present the average hourly source contributions at WC and CC, respectively. From Figures 9.13 and 9.14 it is evident that biomass burning is a significant PM source every hour during the winter. It is also clear that biomass burning is the most significant contributor to both peaks observed in the diurnal cycle. It is not surprising that biomass burning dominated the evening peak, since on cold winter evenings home heating is a necessity, and in Masterton many households rely on wood burners as their main heating source. More surprisingly, biomass burning also dominated the morning PM peak. Trompetter et al. (2010) reported that in larger urban areas the morning peak was anecdotally attributed to motor vehicles, since peak concentrations occur around the morning rush hour, but no firm evidence existed for this suggestion. Based on our source apportionment, it seems likely that Masterton residents are re- 
lighting their fires when they rise in the morning. It was suggested by Trompetter et al. (2010) that the morning peak may also be the result of built-up $\mathrm{PM}_{10}$ at the inversion layer being re-entrained to ground level by atmospheric mixing associated with a break-up of the inversion, which has been observed previously (Aryal et al. 2009). Based on vertical profile measurements taken during the campaign (Trompetter, unpublished results) and ground observations this mechanism was ruled out. We suggest that katabatic flows result in dispersion and a consequent decrease in $\mathrm{PM}_{10}$ concentrations during the early morning and the morning peak results from new particles being emitted as fires are lit or re-stoked in the morning.

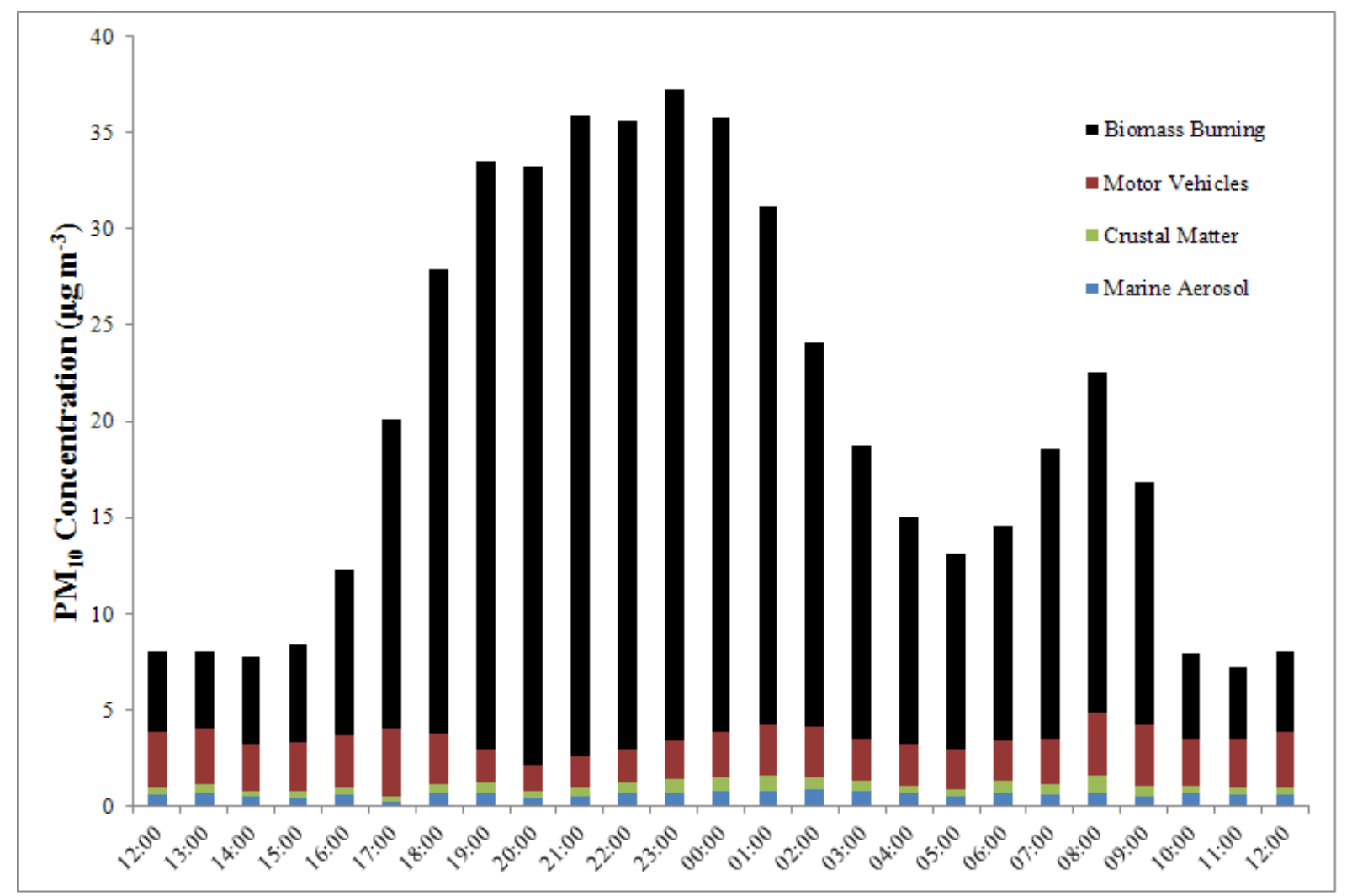

Figure 9.13 Average hourly source contributions at the Wairarapa College site. 


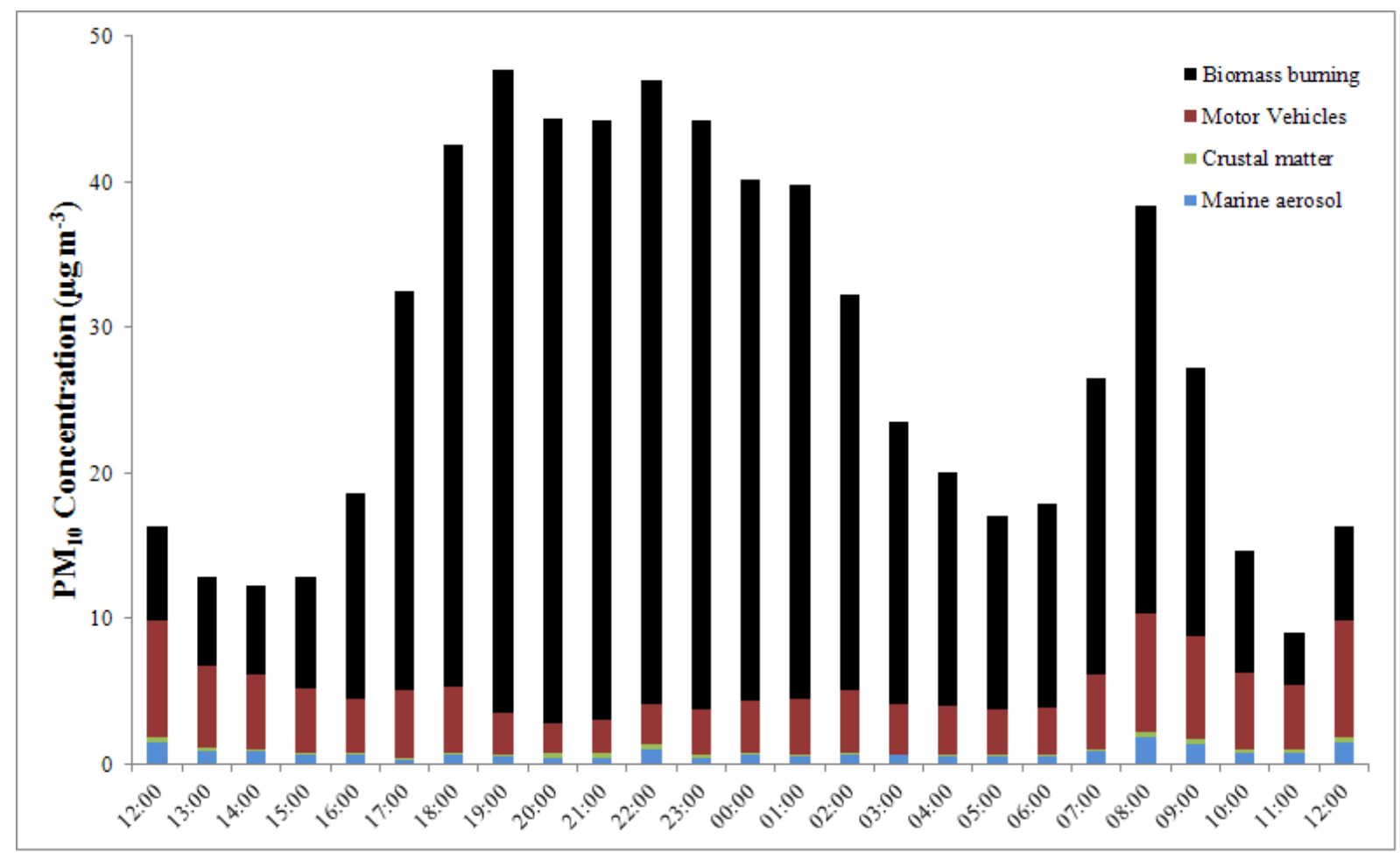

Figure 9.14 Average hourly source contributions at the Chanel College site.

Contributions from the motor vehicle sources at both sites increased during peak traffic hours. At both sites motor vehicle contributions were also evident during hours when traffic would be expected to be minimal, however, Masterton is located along a major highway (State Highway 2) and it is likely that traffic, particularly heavy-duty diesel vehicles, provide a consistent $\mathrm{PM}_{10}$ source, regardless of the time of day. Contributions from the motor vehicle source at the CC site were higher than at the WC site. This is likely because the CC site was closer to the road and in a dense residential area, with more streets in close proximity, than the WC site.

The marine aerosol and crustal matter source contributions at both sites were more pronounced when hourly wind speeds were highest. It is well-known that marine aerosol and crustal matter concentrations increase under increased wind speeds (Fitzgerald 1991), and the results of the hourly source apportionment are consistent with this. Differences in crustal matter contributions between the two sites probably resulted from the slightly different terrains at the two sites. The WC site was located in an open field with little grass cover, leaving the soil highly exposed, while the CC site had much better grass cover and little exposed soil. 


\subsection{Acknowledgements}

The authors thank the Greater Wellington Regional Council for their support and contributions. The authors also thank Bruce Crothers and Ed Hutchinson for their support in the sampling and set-up and Chris Purcell for setting up and maintaining the $2.5 \mathrm{MeV}$ accelerator used for IBA. This work was funded by the Ministry of Science and Innovation under contract C05X0903. 


\section{Nelson: Sources of particulate matter on an hourly time-scale during the winter in a small city}

\subsection{Synopsis}

This chapter has been reproduced in full, as submitted to Environmental Science and Technology, 2012. Ancelet, T., Davy, P. K., Trompetter, W. J., Markwitz, A. and Weatherburn, D. C., 2012. Sources of particulate matter on an hourly time-scale during the winter in a small city. Environmental Science and Technology, submitted.

In this study, PM sources at four different monitoring sites in Nelson, New Zealand were investigated on an hourly time-scale. Three of the sites were located on a horizontal transect, upwind, central and downwind of the general katabatic flow pathway. The fourth monitoring site was located at the central site, but at a height of $26 \mathrm{~m}$, using a knuckleboom, when wind conditions permitted. $\mathrm{PM}_{10}$ concentrations at each of the sites displayed distinct diurnal patterns, with $\mathrm{PM}_{10}$ concentration maxima in the evening (7-11 pm) and in the morning (9-10 am). The central monitoring site experienced consistently higher $\mathrm{PM}_{10}$ concentrations and analysis indicated that inversion events in the confined urban valley were the main factor contributing to the elevated concentrations during the late evening, followed by the dispersion of pollutants by katabatic flows, effectively clearing the boundary layer leading to decreased $\mathrm{PM}_{10}$ concentrations during the early morning. New PM emissions were responsible for the morning peaks in $\mathrm{PM}_{10}$ concentrations. $\mathrm{PM}_{10}$ concentrations at the $26 \mathrm{~m}$ monitoring site were lower than those at ground level. Temperatures and wind speeds were also higher at the elevated site, suggesting that it was located above a shallow inversion layer that limited the vertical mixing of pollutants. Five PM sources were identified for each site: biomass combustion, vehicles, marine aerosol, shipping sulfate and crustal matter. Biomass combustion was the most dominant source at each of the sites and was responsible for both the evening and morning peak $\mathrm{PM}_{10}$ concentrations, suggesting that Nelson residents are re-lighting their fires when they rise in the morning. Prior to this study, contributions from shipping emissions had never been identified in Nelson. 


\subsection{Introduction}

Particulate matter (PM) is well-known to have adverse effects on human health and a range of environmental effects, including local reductions in visibility and effects on the Earth's radiative balance (Dockery et al. 1993; Tsai and Cheng 1999; Nel 2005; Russell and Brunekreef 2009; Pósfai and Buseck 2010). As in many countries, PM concentrations in New Zealand are routinely monitored and the National Environmental Standard (NES) for PM sets a 24-hour average limit for $\mathrm{PM}_{10}$ (PM with aerodynamic diameters less than $10 \mu \mathrm{m}$ ) concentrations at $50 \mu \mathrm{g} \mathrm{m}^{-3}$. Many urban areas in New Zealand exceed the NES numerous times each year, particularly during the winter when wood combustion for home heating is common (http://www.mfe.govt.nz/environmental-reporting/report-cards/air/2009/index.html).

Particulate matter concentrations in New Zealand's urban environments have distinct diurnal cycles, independent of community size or population (Trompetter et al. 2010). During these cycles, $\mathrm{PM}_{10}$ concentrations peak between 10 pm-midnight, with a less pronounced peak also occurring between 8-11 am. These peak concentrations can result in daily $\mathrm{PM}_{10}$ concentrations that exceed the NES. Currently very little is known about PM sources and their contributions on an hourly time-scale, information that is critical for effective air quality management (Ancelet et al. 2012a). To identify the sources and factors contributing to PM pollution episodes on an hourly time-scale, an intensive monitoring campaign was undertaken in Nelson, New Zealand (population 43 000; latitude $-41.16^{\circ}$, longitude $173.17^{\circ}$ ) during the 2011 winter. Nelson is a small city located on the northern coast of New Zealand's South Island. Nelson is known to suffer from poor air quality during the winter, when domestic combustion for home heating is common, and exceeds the NES several times each year, primarily because of the formation of strong temperature inversions that limit the dispersion of pollutants.

We have recently reported the first PM source apportionment study using hourly data obtained from two sampling sites in the rural community of Masterton, New Zealand (Ancelet et al. 2012b). In the current study, hourly coarse ( $\left.\mathrm{PM}_{10-2.5}\right)$ and fine $\left(\mathrm{PM}_{2.5}\right)$ samples were collected from four sites in Nelson and were analyzed for elemental content and black carbon (BC). Hourly $\mathrm{PM}_{10}$ concentrations were determined using continuous $\mathrm{PM}_{10}$ (BAM) monitors and positive matrix factorization (PMF) was used to determine the PM sources and their contributions on an hourly 
time-scale at each site using the hourly elemental, $\mathrm{BC}$ and $\mathrm{PM}_{10}$ data. The results of PMF analyses are directly interpretable as mass contributions from each factor (source) (Paatero and Tapper 1994; Paatero 1997; Song et al. 2001). Two receptor models are available to perform PMF, PMF2 (Paatero 1997) and EPA PMF (USEPA 2008). EPA PMF adopts a bilinear model which is solved by the Multilinear Engine (Paatero 1999) and incorporates a graphical user interface. When compared with PMF2, EPA PMF has been shown to provide similar results with some minor differences in the final solutions (Kim and Hopke 2007).

Using hourly source contributions and meteorological data from each of the sites, potential PM transport mechanisms were also identified. High-temporal resolution source apportionment studies can provide unique and highly relevant information for the implementation of PM mitigation strategies.

\subsection{Experimental Section}

\subsubsection{Sample Collection}

Ambient air monitoring was conducted at four locations in an urban valley in Nelson. Three of the sites were located along the general katabatic flow pathway (upwind, central and downwind) (Manins and Sawford 1979). The fourth site (Aloft) was located alongside the central site, but was raised to a height of $26 \mathrm{~m}$ above the ground level site using a knuckleboom (when wind conditions permitted). This type of experimental setup has not been reported before and Figure 10.1 presents a schematic illustration of the sampling site locations. The sampling site locations were designed to provide an indication of PM transport horizontally and vertically within the Nelson airshed. 


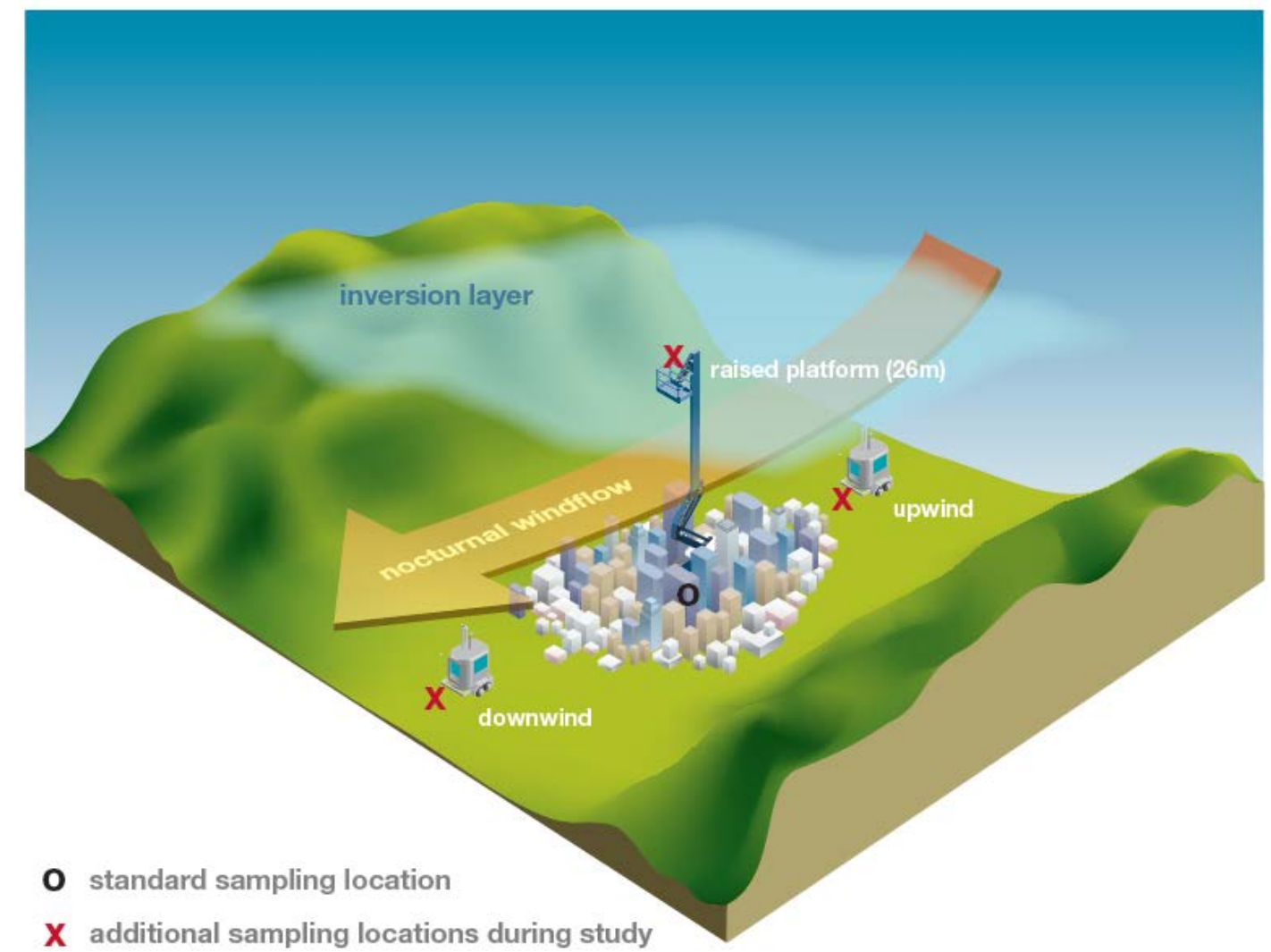

Figure 10.1 Schematic illustration of the sampling site locations in Nelson. In the Nelson case, the upwind site was located on the left, while the downwind site was located on the right.

The upwind site was located on the grounds of the Nelson Intermediate School (NIS) (latitude $-41.172266^{\circ}$, longitude $173.260500^{\circ}$ ). The NIS site was situated approximately $1.4 \mathrm{~km}$ southwest of the central site and $90 \mathrm{~m}$ from the nearest road. The central (NCC) and elevated (Aloft) sites were co-located with a Nelson City Council ambient air quality monitoring station used for compliance monitoring (latitude $-41.164150^{\circ}$, latitude $173.162447^{\circ}$ ) and were approximately $90 \mathrm{~m}$ from the nearest road. The downwind site was located within the Nelson Fire Station compound (NFS) (latitude $-41.162241^{\circ}$, longitude $173.164400^{\circ}$ ) and was approximately $600 \mathrm{~m}$ north of the NCC site and $60 \mathrm{~m}$ from the nearest road. The land around each of the sites was flat and surrounded by open space or buildings no more than two stories high. Figure 10.2 presents the locations of each of the sampling sites. 


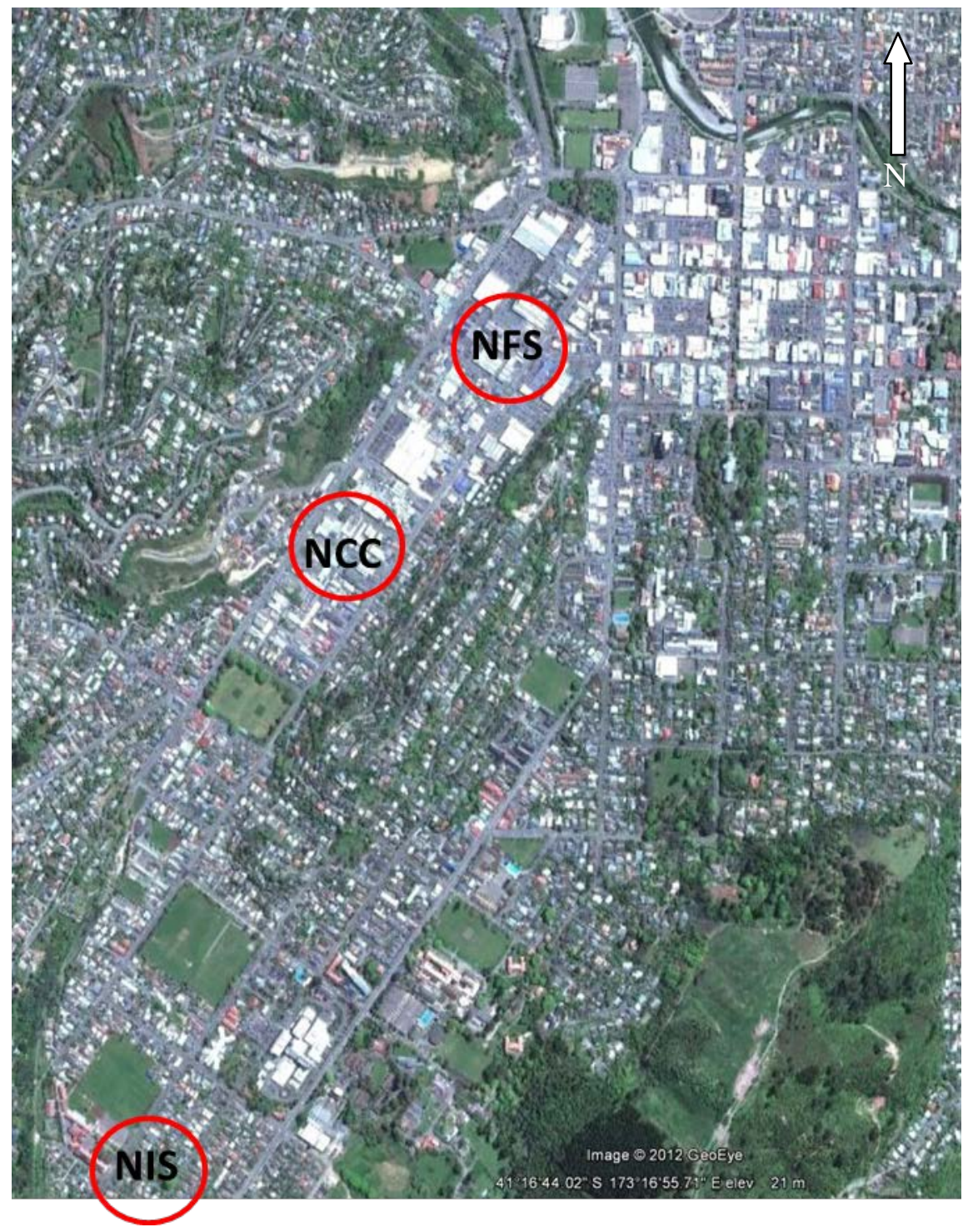

Figure 10.2 Locations of the sampling sites within Nelson (NIS: Nelson Intermediate School (upwind); NCC: Nelson City Council (central); NFS: Nelson Fire Station (downwind)). The Aloft site was co-located with the NCC site.

Each site was equipped with a Streaker sampler (PIXE International Corporation, USA), an E-BAM (Met One Instruments, Inc.) and a meteorological station (Vaisala WXT520) in the same fashion as previously reported (Ancelet et al. 2012b). In this study, a total of 47 samples, or 47 hours, were collected on each set of size-resolved ( $\mathrm{PM}_{10-2.5}$ and $\left.\mathrm{PM}_{2.5}\right)$ filters. The monitoring program ran from JuneAugust 2011 and a total of 6896 samples were collected among the four sites (2162 from NIS, 2068 from NCC, 1632 from NFS and 1034 (414 when raised to $26 \mathrm{~m}$ ) from the Aloft site). 


\subsubsection{Elemental Analysis}

Ion beam analysis (IBA) was used to measure the concentrations of elements with atomic numbers above neon in the PM collected. IBA measurements were carried out at the New Zealand Ion Beam Analysis Facility operated by the Institute of Geological and Nuclear Sciences (GNS) in Gracefield, Lower Hutt, New Zealand (Trompetter et al. 2005). Further details on the IBA techniques used, analytical uncertainties and limits of detection have been reported previously (Ancelet et al. 2012b). Black carbon was measured using a M43D Digital Smoke Stain Reflectometer (Ancelet et al. 2011b). Prior to the PMF analyses, data and uncertainty matrices were prepared for each site in the same manner as previous studies (Polissar et al. 1998; Song et al. 2001).

\subsubsection{Receptor Modeling}

Receptor modeling and apportionment of PM mass by PMF was performed using the EPAPMF version 3.0.2.2 program in accordance with the User's Guide (USEPA 2008). With PMF, sources are constrained to have non-negative species concentrations, no sample can have a negative source contribution and error estimates for each observed point are used as point-by-point weights. This is a distinct advantage of PMF, since it can accommodate missing or below detection limit data that is a common feature of environmental monitoring (Song et al. 2001). Data screening and the source apportionment were performed in the same manner as previously reported (Ancelet et al. 2012b). Table 10.1 provides a summary of the parameters used for the PMF analyses and the diagnostics obtained for each of the sites. 
Table 10.1 Parameters used for PMF analyses and diagnostics obtained from the analyses for each of the sites

\begin{tabular}{|c|c|c|c|c|}
\hline & $\begin{array}{c}\text { Nelson Intermediate } \\
\text { School }\end{array}$ & Nelson City Council & Nelson Fire Station & Aloft \\
\hline $\begin{array}{l}\text { Extra modeling } \\
\text { uncertainty (\%) }\end{array}$ & 5 & 5 & 5 & 5 \\
\hline $\begin{array}{l}\text { Number of base } \\
\text { runs }\end{array}$ & 20 & 20 & 20 & 20 \\
\hline Base run seed & Random & Random & Random & Random \\
\hline $\begin{array}{c}\text { Number of } \\
\text { bootstraps }\end{array}$ & 200 & 200 & 200 & 200 \\
\hline $\begin{array}{c}\text { Minimum } \\
\text { correlation R-value }\end{array}$ & 0.6 & 0.6 & 0.6 & 0.6 \\
\hline Bootstrap seed & Random & Random & Random & Random \\
\hline Block size & 24 & 24 & 24 & 24 \\
\hline Theoretical Q & 9729 & 10685 & 7344 & 5327 \\
\hline Robust Q & 7510 & 9433 & 5304 & 3633 \\
\hline True Q & 7831 & 9861 & 5426 & 3853 \\
\hline $\begin{array}{c}\text { Number of } \\
\text { bootstrapped factors } \\
\text { mapped to original } \\
\text { factor } 1\end{array}$ & 200 & 190 & 199 & 200 \\
\hline $\begin{array}{c}\text { Number of } \\
\text { bootstrapped factors } \\
\text { mapped to original } \\
\text { factor } 2 \\
\end{array}$ & 190 & 200 & 200 & 200 \\
\hline $\begin{array}{c}\text { Number of } \\
\text { bootstrapped factors } \\
\text { mapped to original } \\
\text { factor } 3\end{array}$ & 200 & 197 & 200 & 200 \\
\hline $\begin{array}{c}\text { Number of } \\
\text { bootstrapped factors } \\
\text { mapped to original } \\
\text { factor } 4\end{array}$ & 200 & 200 & 188 & 194 \\
\hline $\begin{array}{c}\text { Number of } \\
\text { bootstrapped factors } \\
\text { mapped to original } \\
\text { factor } 5\end{array}$ & 192 & 187 & 195 & 200 \\
\hline $\begin{array}{c}\text { Number of } \\
\text { bootstrapped factors } \\
\text { mapped to no } \\
\text { original factor }\end{array}$ & 3 & 9 & 7 & 5 \\
\hline Maximum D value & $<0.1$ & 0.1 & 0.1 & $<0.1$ \\
\hline
\end{tabular}

\subsection{Results and Discussion}

\subsection{1 $\mathrm{PM}_{10}$ Concentrations}

$\mathrm{PM}_{10}$ concentrations at each of the sites displayed distinct diurnal cycles. Figure 10.3 (a) presents the average hourly PM $_{10}$ concentrations at the NIS, NCC and NFS sites over the entire sampling period. A number of features are apparent from Figure 10.3 (a). First, the diurnal profiles of the three sites are very similar, with peak $\mathrm{PM}_{10}$ concentrations occurring between 7-11 pm and 9-10 am. Second, $\mathrm{PM}_{10}$ concentrations at each of the sites decreased in similar fashions, suggesting a common 
PM sink or dispersion mechanism among the sites. Finally, hourly $\mathrm{PM}_{10}$ concentrations during peak hours at NCC were higher than at NIS and NFS.

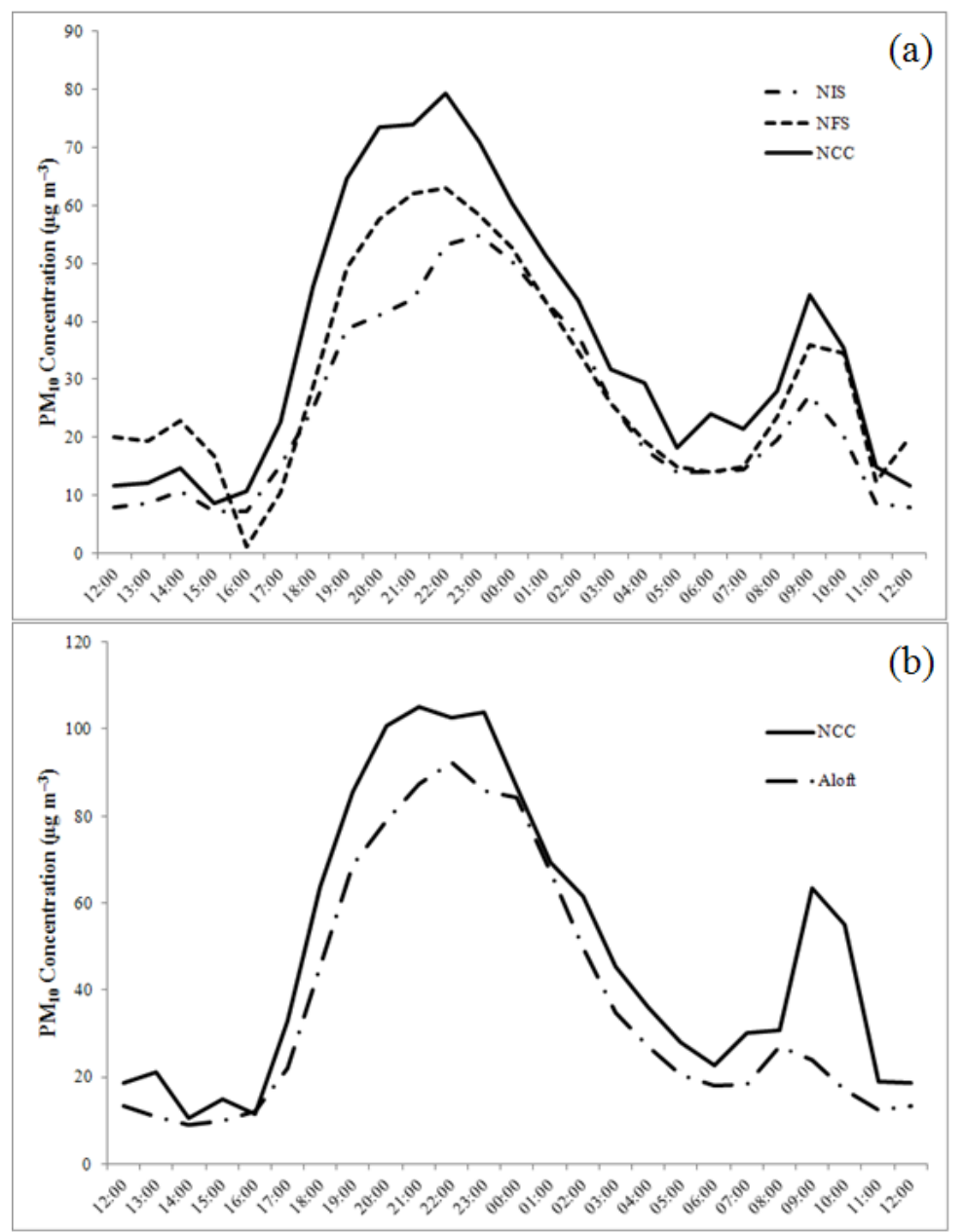

Figure 10.3 Average hourly $\mathrm{PM}_{10}$ concentrations at (a) the Nelson Intermediate School, Nelson City Council and Nelson Fire Station sites and (b) at the Nelson City Council and Aloft sites during periods when the knuckleboom was raised.

Figure 10.3 (b) presents the average hourly $\mathrm{PM}_{10}$ concentrations at the NCC and Aloft sites during periods when the knuckleboom was raised (212 hours total). Since the knuckleboom was only raised when wind speeds were low (during anticyclonic atmospheric conditions), $\mathrm{PM}_{10}$ concentrations measured at the NCC site were higher than during periods when the knuckleboom was not raised. Average hourly $\mathrm{PM}_{10}$ concentrations at both sites featured peak $\mathrm{PM}_{10}$ concentrations occurring between 8 pm-midnight. A small morning peak ( $8 \mathrm{am})$ in $\mathrm{PM}_{10}$ concentrations at the Aloft site was apparent, while the NCC site featured a much larger morning peak in $\mathrm{PM}_{10}$ concentrations between 9-10 am. The higher concentrations measured, particularly during the morning, at the ground level NCC site indicated the formation of a shallow inversion layer that limited the vertical dispersion of $\mathrm{PM}_{10}$. Supporting 
this conclusion, average wind speeds and air temperatures at the Aloft site were higher than at the ground-based NCC site during hours with peak $\mathrm{PM}_{10}$ concentrations.

Comparisons of daily average $\mathrm{PM}_{10}$ concentrations among the four sites revealed that NIS did not exceed the New Zealand NES for $\mathrm{PM}_{10}$ of $50 \mathrm{\mu g} \mathrm{m}^{-3}$ on any day during the study period, while the NFS, Aloft and NCC sites had 5, 2 and 15 exceedances, respectively, during this study. It is important to note that E-BAMs are not certified for air quality compliance monitoring, but it is clear that the sampling location, both horizontally and vertically, can have a significant impact on measured $\mathrm{PM}_{10}$ concentrations in Nelson.

The local meteorology in Nelson was investigated on an hourly basis to gain a better understanding of PM transport. Figure 10.4 presents a wind rose plot over the entire sampling period from NCC. Wind rose plots for the other sites were nearly identical to that from NCC. Figure 10.4 shows that winds during the sampling period were predominantly from the southwest, with smaller contributions from the northeast, in line with the direction of the valley. Using the high temporal resolution data available in this study, hourly pollution roses for each of the sites were developed using the $\mathrm{R}$ statistical and Openair packages (Carslaw and Ropkins 2011; R Development Core Team 2011). Figure 10.5 presents hourly $\mathrm{PM}_{10}$ pollution roses for the NCC site. Figure 10.5 suggests that the elevated $\mathrm{PM}_{10}$ concentrations measured at NCC were the result of the transport of $\mathrm{PM}_{10}$ by katabatic flows from the southwest along the downslope contour of the valley. According to this mechanism, it would be expected that $\mathrm{PM}_{10}$ concentrations at the NFS site, which was further downwind than the NCC site, would be the highest of all the sites. From Figure 10.3 (a) it is clear that this is not the case. We suggest that this results from both the urban valley opening up just before the NFS site, allowing for more effective dispersion conditions that result in lower $\mathrm{PM}_{10}$ concentrations and a reduction in the number of local emission sources as largely residential areas turn into commercial areas near the NFS site. 


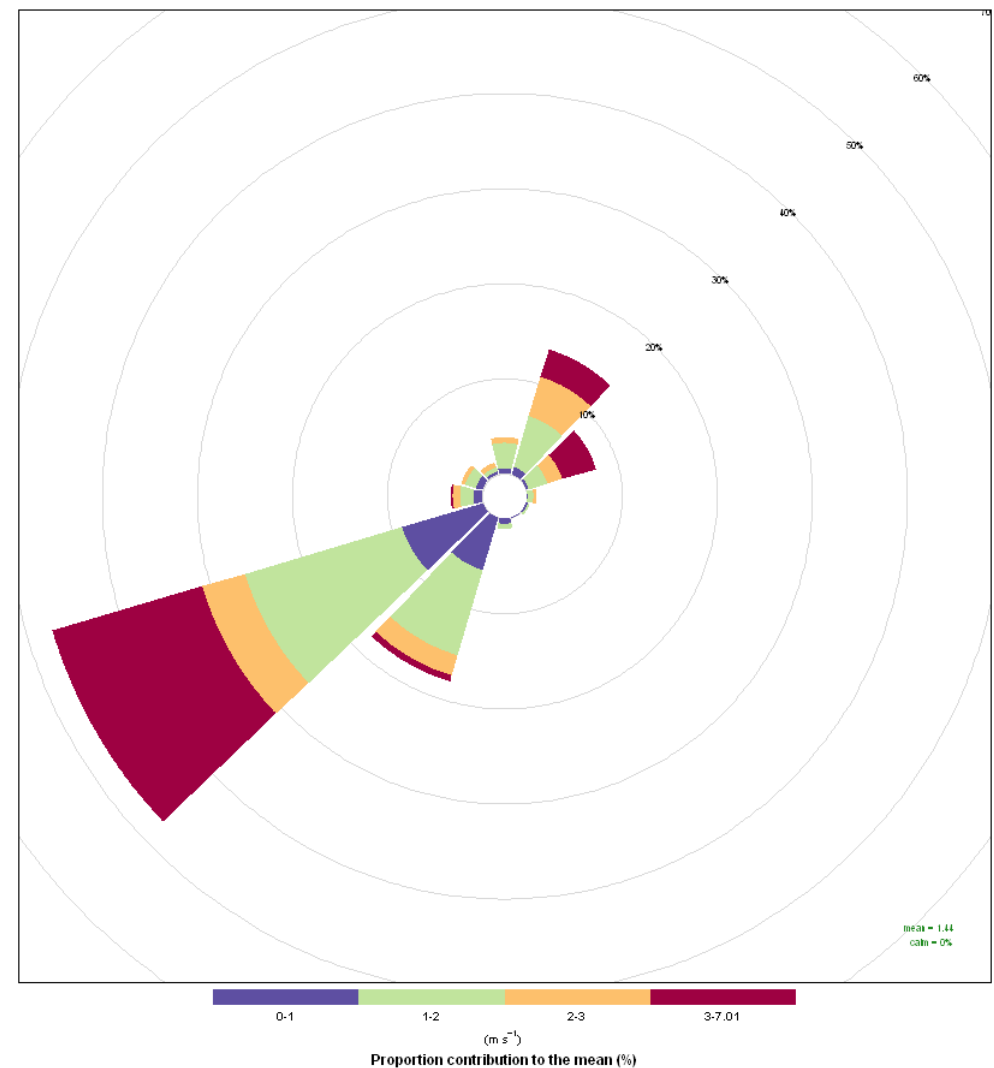

Figure 10.4 Wind rose plot over the entire sampling period from the Nelson City Council site. The radial dimensions indicate the frequency (\%) of winds from each direction. 


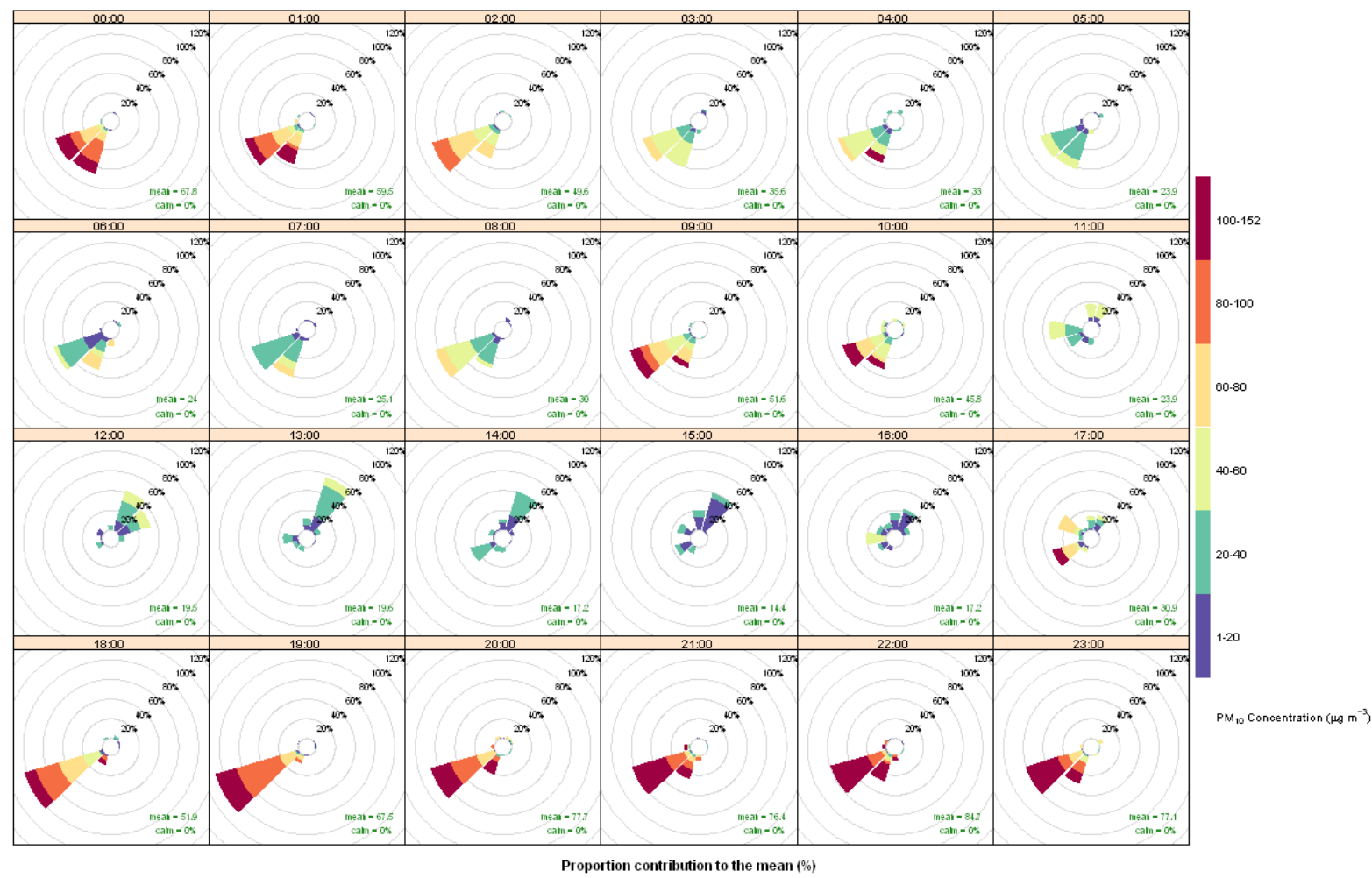

Figure 10.5 Hourly pollution roses from the Nelson City Council site indicating wind directions contributing the most to average hourly $\mathrm{PM}_{10}$ concentrations. The radial dimensions indicate the percentage of the total pollution that arrives from each wind sector during each one-hour period.

\subsubsection{Sources of Ambient $P M_{10}$}

Tables 10.2, 10.3, 10.4 and 10.5 present the average, maximum and median hourly concentrations of species used for source apportionment at the NIS, NCC, NFS and Aloft sites, respectively, along with standard deviations, average uncertainties, average limits of detection and the number of samples above the limit of detection for each species. Reconstructed masses (RCMs) determined using the elemental data accounted for 19, 21, 19 and $18 \%$ of the $\mathrm{PM}_{10}$ mass at the NIS, NCC, NFS and Aloft sites, respectively (Malm et al. 1994). Because numerous species, including organic carbon (OC) were not quantified and shipping sulfate was not accounted for, the relatively low RCMs are not surprising. Fine $\mathrm{Al}$ and $\mathrm{Si}$ were quantified, but the values were not used because the filters, used as received, were unevenly contaminated with $\mathrm{Al}$ and $\mathrm{Si}$, so a background correction could not be performed without unintentionally affecting species variance within the dataset. Marine aerosol (a source featuring little contribution from unmeasured species) RCMs were close to those identified from PMF. 
Table 10.2 $\mathrm{PM}_{10}\left(\mu \mathrm{g} \mathrm{m}^{-3}\right)$, BC $\left(\mathrm{ng} \mathrm{m}^{-3}\right)$ and elemental $\left(\mathrm{ng} \mathrm{m}^{-3}\right)$ data from the Nelson Intermediate School site (1081 samples)

\begin{tabular}{|c|c|c|c|c|c|c|c|}
\hline & Average & Maximum & Median & $\begin{array}{l}\text { Standard } \\
\text { Deviation }\end{array}$ & $\begin{array}{c}\text { Average } \\
\text { Uncertainty }\end{array}$ & $\begin{array}{c}\text { Average } \\
\text { LOD } \\
\end{array}$ & $\begin{array}{c}\text { Number }> \\
\text { LOD } \\
\end{array}$ \\
\hline $\mathrm{PM}_{10}$ & 27 & 121 & 19 & 25 & & & \\
\hline Fine BC & 5203 & 14444 & 4489 & 2376 & 565 & 800 & 1081 \\
\hline Fine $S$ & 228 & 2357 & 217 & 128 & 23 & 30 & 1081 \\
\hline Fine $\mathrm{K}$ & 155 & 1051 & 118 & 137 & 22 & 30 & 930 \\
\hline Fine Ca & 72 & 566 & 62 & 49 & 21 & 26 & 990 \\
\hline Fine V & 4 & 48 & 0 & 6 & 17 & 23 & 92 \\
\hline Fine Fe & 37 & 366 & 31 & 29 & 9 & 11 & 966 \\
\hline Fine $\mathrm{Cu}$ & 5 & 142 & 3 & 10 & 11 & 15 & 122 \\
\hline Fine Zn & 15 & 273 & 10 & 25 & 13 & 17 & 351 \\
\hline $\begin{array}{c}\text { Coarse } \\
\mathrm{Al}\end{array}$ & 143 & 2878 & 120 & 161 & 42 & 65 & 938 \\
\hline $\begin{array}{c}\text { Coarse } \\
\text { Si }\end{array}$ & 553 & 3616 & 505 & 251 & 37 & 45 & 1081 \\
\hline $\begin{array}{c}\text { Coarse } \\
\text { S }\end{array}$ & 36 & 818 & 29 & 44 & 23 & 35 & 499 \\
\hline $\begin{array}{c}\text { Coarse } \\
\mathrm{Cl}\end{array}$ & 168 & 2451 & 71 & 274 & 24 & 33 & 794 \\
\hline $\begin{array}{c}\text { Coarse } \\
\mathrm{K} \\
\end{array}$ & 33 & 580 & 26 & 35 & 22 & 34 & 459 \\
\hline $\begin{array}{c}\text { Coarse } \\
\mathrm{Ca} \\
\end{array}$ & 202 & 662 & 192 & 53 & 24 & 27 & 1081 \\
\hline $\begin{array}{c}\text { Coarse } \\
\mathrm{Fe} \\
\end{array}$ & 50 & 1387 & 31 & 73 & 10 & 12 & 905 \\
\hline $\begin{array}{c}\text { Coarse } \\
\mathrm{Cu}\end{array}$ & 9 & 1110 & 0 & 52 & 13 & 16 & 137 \\
\hline $\begin{array}{c}\text { Coarse } \\
\text { Zn }\end{array}$ & 10 & 657 & 4 & 39 & 14 & 18 & 137 \\
\hline
\end{tabular}


Table 10.3 $\quad \mathrm{PM}_{10}\left(\mu \mathrm{g} \mathrm{m}^{-3}\right), \mathrm{BC}\left(\mathrm{ng} \mathrm{m}^{-3}\right)$ and elemental $\left(\mathrm{ng} \mathrm{m}^{-3}\right)$ data from the Nelson City Council site (1034 samples)

\begin{tabular}{|c|c|c|c|c|c|c|c|}
\hline & Average & Maximum & Median & $\begin{array}{l}\text { Standard } \\
\text { Deviation } \\
\end{array}$ & $\begin{array}{c}\text { Average } \\
\text { Uncertainty } \\
\end{array}$ & $\begin{array}{c}\text { Average } \\
\text { LOD } \\
\end{array}$ & $\begin{array}{c}\text { Number }> \\
\text { LOD }\end{array}$ \\
\hline $\mathrm{PM}_{10}$ & 40 & 152 & 29 & 35 & & & \\
\hline Fine BC & 5514 & 16061 & 4516 & 3081 & 547 & 800 & 1034 \\
\hline Fine $S$ & 305 & 1796 & 269 & 174 & 22 & 31 & 1034 \\
\hline Fine K & 227 & 1077 & 161 & 206 & 24 & 29 & 919 \\
\hline Fine Ca & 86 & 641 & 82 & 47 & 22 & 27 & 974 \\
\hline Fine V & 5 & 40 & 0 & 7 & 19 & 22 & 98 \\
\hline Fine Fe & 52 & 455 & 38 & 49 & 11 & 14 & 999 \\
\hline Fine $\mathrm{Cu}$ & 5 & 104 & 3 & 7 & 10 & 13 & 105 \\
\hline Fine Zn & 21 & 430 & 14 & 35 & 14 & 18 & 352 \\
\hline $\begin{array}{c}\text { Coarse } \\
\mathrm{Al}\end{array}$ & 188 & 3835 & 145 & 210 & 44 & 64 & 947 \\
\hline $\begin{array}{l}\text { Coarse } \\
\text { Si }\end{array}$ & 735 & 3867 & 631 & 430 & 35 & 42 & 1034 \\
\hline $\begin{array}{c}\text { Coarse } \\
\mathrm{S} \\
\end{array}$ & 59 & 769 & 42 & 64 & 21 & 38 & 522 \\
\hline $\begin{array}{c}\text { Coarse } \\
\mathrm{Cl}\end{array}$ & 285 & 3864 & 111 & 460 & 23 & 35 & 784 \\
\hline $\begin{array}{c}\text { Coarse } \\
\mathrm{K} \\
\end{array}$ & 51 & 1056 & 39 & 61 & 20 & 31 & 464 \\
\hline $\begin{array}{c}\text { Coarse } \\
\mathrm{Ca} \\
\end{array}$ & 239 & 1466 & 209 & 105 & 25 & 29 & 1034 \\
\hline $\begin{array}{c}\text { Coarse } \\
\mathrm{Fe}\end{array}$ & 91 & 1289 & 52 & 122 & 11 & 14 & 894 \\
\hline $\begin{array}{c}\text { Coarse } \\
\mathrm{Cu}\end{array}$ & 6 & 517 & 0 & 21 & 15 & 13 & 121 \\
\hline $\begin{array}{c}\text { Coarse } \\
\text { Zn } \\
\end{array}$ & 11 & 1078 & 6 & 40 & 16 & 14 & 134 \\
\hline
\end{tabular}


Table 10.4 $\quad \mathrm{PM}_{10}\left(\mu \mathrm{g} \mathrm{m}^{-3}\right)$, BC $\left(\mathrm{ng} \mathrm{m}^{-3}\right)$ and elemental $\left(\mathrm{ng} \mathrm{m}^{-3}\right)$ data from the Nelson Fire Station site (816 samples)

\begin{tabular}{|c|c|c|c|c|c|c|c|}
\hline & Average & Maximum & Median & $\begin{array}{l}\text { Standard } \\
\text { Deviation }\end{array}$ & $\begin{array}{c}\text { Average } \\
\text { Uncertainty } \\
\end{array}$ & $\begin{array}{c}\text { Average } \\
\text { LOD } \\
\end{array}$ & $\begin{array}{c}\text { Number }> \\
\text { LOD }\end{array}$ \\
\hline $\mathrm{PM}_{10}$ & 32 & 160 & 21 & 30 & & & \\
\hline Fine BC & 5049 & 15612 & 4266 & 2427 & 551 & 800 & 815 \\
\hline Fine $S$ & 202 & 886 & 190 & 91 & 25 & 33 & 816 \\
\hline Fine $\mathrm{K}$ & 136 & 1677 & 95 & 143 & 21 & 25 & 786 \\
\hline Fine $\mathrm{Ca}$ & 64 & 464 & 60 & 38 & 25 & 26 & 801 \\
\hline Fine V & 5 & 39 & 1 & 7 & 21 & 24 & 100 \\
\hline Fine $\mathrm{Fe}$ & 36 & 294 & 31 & 24 & 13 & 16 & 800 \\
\hline Fine $\mathrm{Cu}$ & 5 & 79 & 4 & 8 & 12 & 15 & 99 \\
\hline Fine $\mathrm{Zn}$ & 15 & 236 & 10 & 20 & 15 & 17 & 361 \\
\hline $\begin{array}{c}\text { Coarse } \\
\mathrm{Al}\end{array}$ & 124 & 2005 & 105 & 157 & 41 & 59 & 801 \\
\hline $\begin{array}{c}\text { Coarse } \\
\text { Si }\end{array}$ & 465 & 1768 & 429 & 156 & 34 & 45 & 816 \\
\hline $\begin{array}{l}\text { Coarse } \\
\text { S }\end{array}$ & 43 & 631 & 31 & 59 & 23 & 33 & 411 \\
\hline $\begin{array}{c}\text { Coarse } \\
\mathrm{Cl}\end{array}$ & 279 & 10967 & 79 & 852 & 22 & 34 & 802 \\
\hline $\begin{array}{c}\text { Coarse } \\
\mathrm{K}\end{array}$ & 36 & 399 & 27 & 39 & 19 & 32 & 655 \\
\hline $\begin{array}{c}\text { Coarse } \\
\mathrm{Ca}\end{array}$ & 202 & 779 & 191 & 60 & 21 & 27 & 808 \\
\hline $\begin{array}{c}\text { Coarse } \\
\text { Fe }\end{array}$ & 70 & 623 & 45 & 76 & 12 & 15 & 812 \\
\hline $\begin{array}{c}\text { Coarse } \\
\mathrm{Cu}\end{array}$ & 7 & 329 & 0 & 21 & 17 & 19 & 103 \\
\hline $\begin{array}{c}\text { Coarse } \\
\mathrm{Zn} \\
\end{array}$ & 9 & 430 & 5 & 24 & 14 & 17 & 122 \\
\hline
\end{tabular}


Table 10.5 $\quad \mathrm{PM}_{10}\left(\mu \mathrm{g} \mathrm{m}^{-3}\right)$, BC $\left(\mathrm{ng} \mathrm{m}^{-3}\right)$ and elemental $\left(\mathrm{ng} \mathrm{m} \mathrm{m}^{-3}\right)$ data from the Aloft site (517 samples)

\begin{tabular}{|c|c|c|c|c|c|c|c|}
\hline & Average & Maximum & Median & $\begin{array}{l}\text { Standard } \\
\text { Deviation } \\
\end{array}$ & $\begin{array}{c}\text { Average } \\
\text { Uncertainty }\end{array}$ & $\begin{array}{c}\text { Average } \\
\text { LOD }\end{array}$ & $\begin{array}{c}\text { Number }> \\
\text { LOD } \\
\end{array}$ \\
\hline $\mathrm{PM}_{10}$ & 37 & 129 & 24 & 33 & & & \\
\hline Fine BC & 6136 & 21105 & 4897 & 3525 & 555 & 800 & 517 \\
\hline Fine $\mathrm{S}$ & 282 & 2766 & 241 & 217 & 25 & 33 & 516 \\
\hline Fine K & 235 & 1168 & 162 & 208 & 24 & 31 & 511 \\
\hline Fine $\mathrm{Ca}$ & 63 & 338 & 57 & 38 & 19 & 28 & 498 \\
\hline Fine V & 5 & 51 & 1 & 7 & 19 & 21 & 103 \\
\hline Fine Fe & 44 & 362 & 34 & 39 & 11 & 12 & 512 \\
\hline Fine $\mathrm{Cu}$ & 6 & 81 & 4 & 10 & 10 & 17 & 97 \\
\hline Fine Zn & 21 & 365 & 13 & 34 & 11 & 16 & 398 \\
\hline $\begin{array}{c}\text { Coarse } \\
\mathrm{Al}\end{array}$ & 146 & 1963 & 116 & 192 & 46 & 68 & 506 \\
\hline $\begin{array}{c}\text { Coarse } \\
\text { Si }\end{array}$ & 453 & 1823 & 415 & 176 & 41 & 48 & 517 \\
\hline $\begin{array}{c}\text { Coarse } \\
\mathrm{S}\end{array}$ & 37 & 814 & 31 & 49 & 26 & 37 & 334 \\
\hline $\begin{array}{c}\text { Coarse } \\
\mathrm{Cl}\end{array}$ & 125 & 1209 & 70 & 158 & 21 & 29 & 488 \\
\hline $\begin{array}{c}\text { Coarse } \\
\text { K }\end{array}$ & 26 & 99 & 24 & 21 & 20 & 31 & 322 \\
\hline $\begin{array}{c}\text { Coarse } \\
\mathrm{Ca}\end{array}$ & 202 & 1100 & 187 & 74 & 25 & 29 & 434 \\
\hline $\begin{array}{c}\text { Coarse } \\
\text { Fe }\end{array}$ & 56 & 573 & 38 & 64 & 13 & 17 & 477 \\
\hline $\begin{array}{c}\text { Coarse } \\
\mathrm{Cu}\end{array}$ & 7 & 742 & 0 & 34 & 11 & 15 & 128 \\
\hline $\begin{array}{c}\text { Coarse } \\
\mathrm{Zn}\end{array}$ & 18 & 865 & 5 & 178 & 11 & 14 & 113 \\
\hline
\end{tabular}

The application of PMF to hourly elemental data from each of the sites revealed five $\mathrm{PM}_{10}$ sources at each of the sites. The source profiles obtained for NCC are presented in Figure 10.6, while the source profiles for NIS, NFS and Aloft are presented as Figures 10.7, 10.8 and 10.9, respectively. The error bars shown in Figures 10.6, 10.7, 10.8 and 10.9 indicate standard deviations determined from bootstrapping in the EPAPMF program. Limitations in the bootstrapping technique have previously been discussed (Ancelet et al. 2012b). 


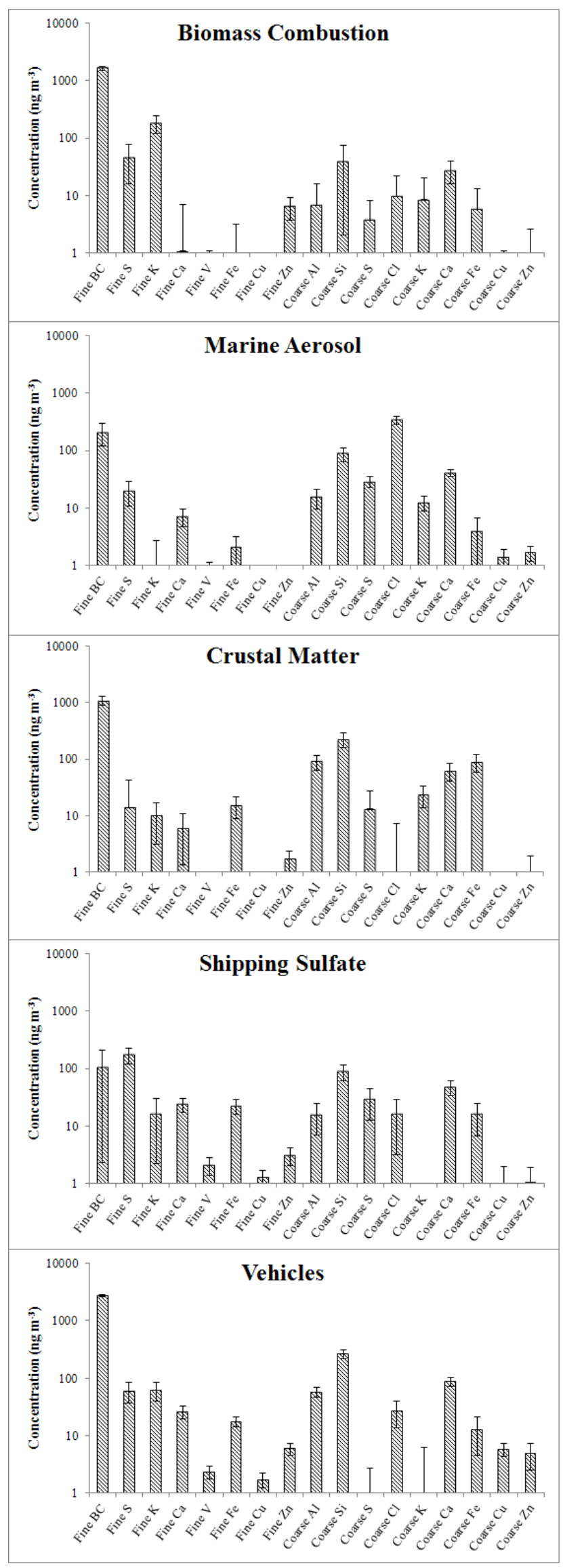

Figure 10.6 Source profiles obtained at the Nelson City Council site. 


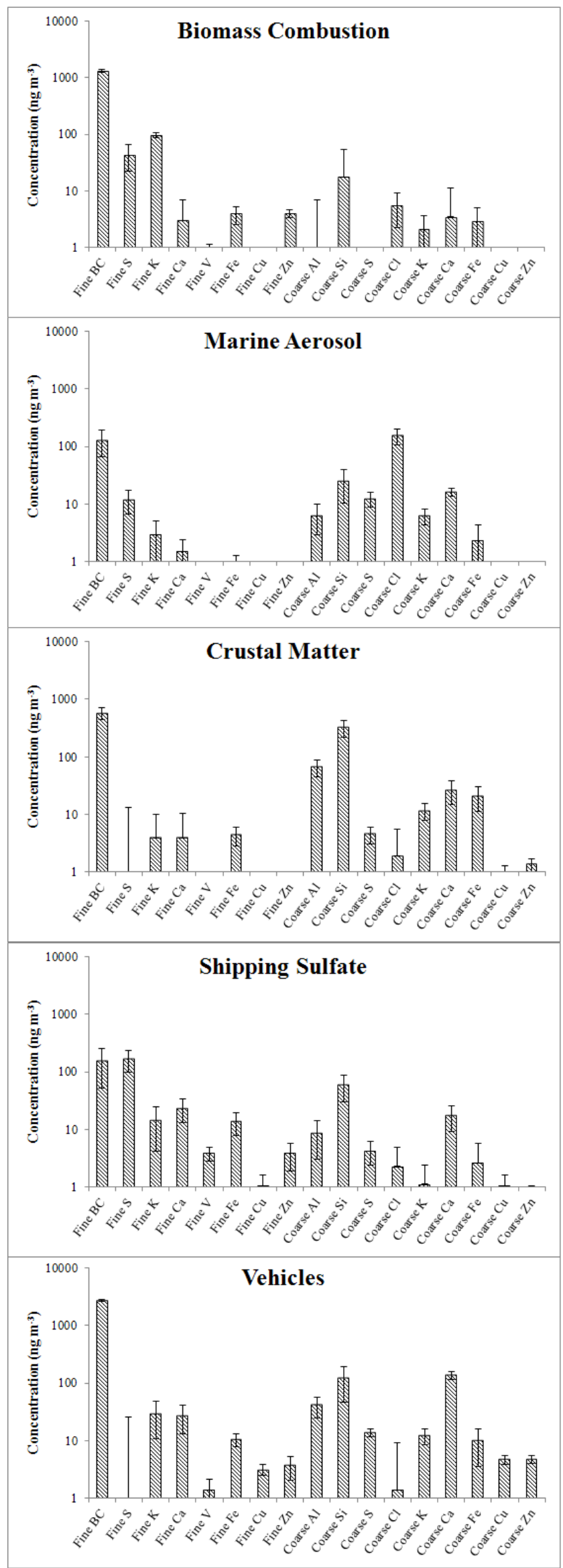

Figure 10.7 Source profiles obtained at the Nelson Intermediate School site. 


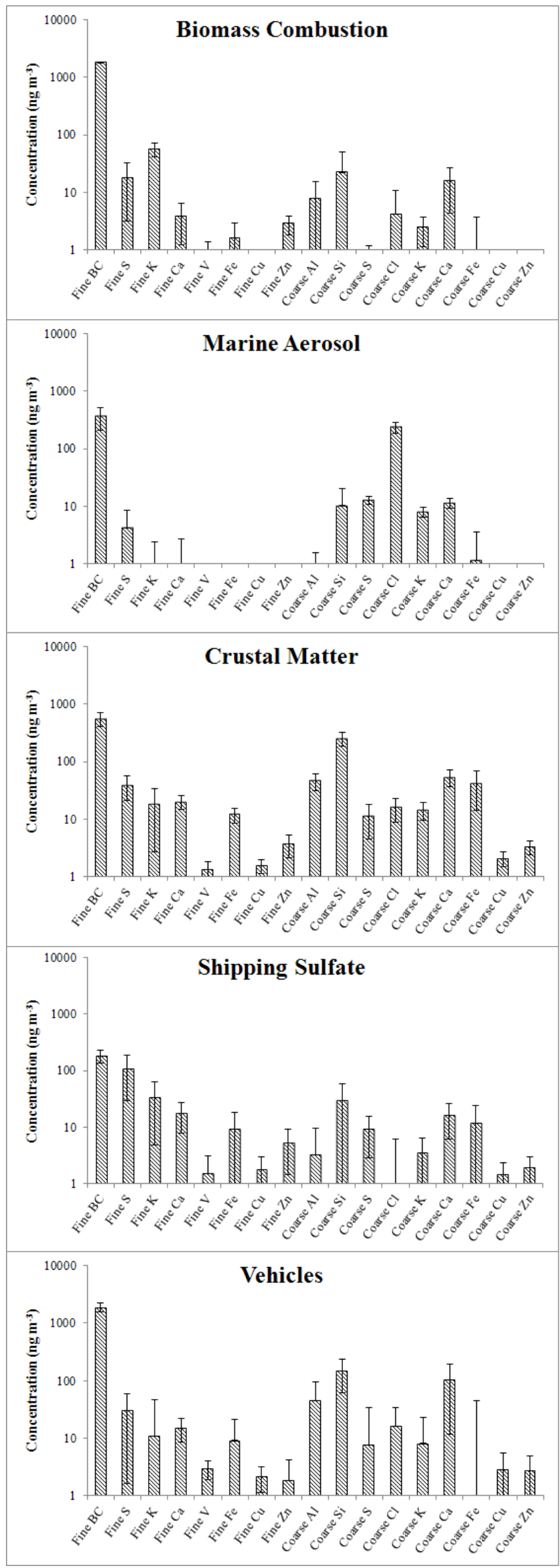

Figure 10.8 Source profiles obtained at the Nelson Fire Station site. 


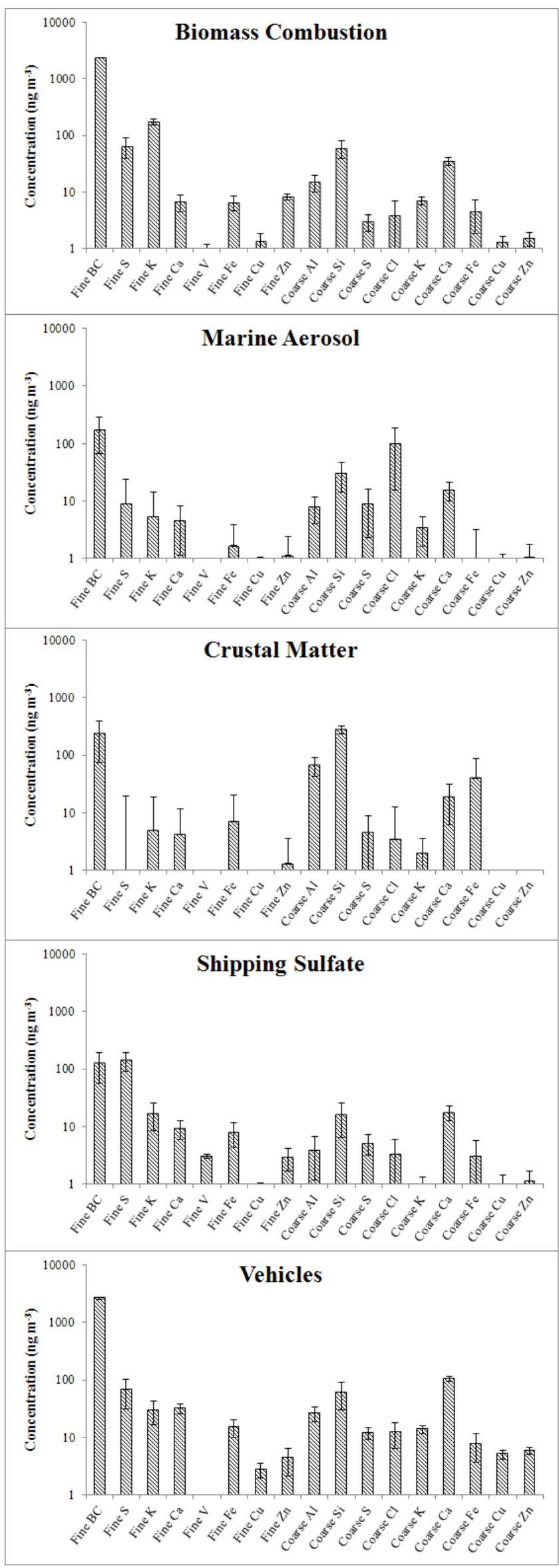

Figure 10.9 Source profiles obtained at the Aloft site. 
The sources presented in Figures 10.6, 10.7, 10.8 and 10.9 were found to explain 97, 96, 98 and 98\% of the $\mathrm{PM}_{10}$ mass measured by the E-BAMS at NIS, NCC, NFS and Aloft, respectively, after regression using $\mathrm{PM}_{10}$ concentrations. Factor one contributed to $86,87,89$ and $85 \%$ of the $\mathrm{PM}_{10}$ mass at NIS, NCC, NFS and Aloft, respectively. It was identified as a biomass combustion source because of high BC and fine K loadings. Potassium is usually used alongside BC as a marker for biomass burning, and wood combustion in particular (Fine et al. 2002; Khalil and Rasmussen 2003).

Factor two accounted for 9, 3, 5 and $8 \%$ of the PM 10 mass at NIS, NCC, NFS and Aloft, respectively. This factor was characterized as a vehicular source, which included vehicular exhaust and non-exhaust emissions, such as road dust and brake wear. Road dust is generated by the turbulent passage of vehicles over local roads and the source profiles feature crustal elements ( $\mathrm{Al}$ and $\mathrm{Si}$ ) enriched with $\mathrm{BC}$, $\mathrm{Ca}$ and $\mathrm{Fe}$. The vehicle source profiles reported here are consistent with those reported previously (Garg et al. 2000; Schauer et al. 2006). Black carbon in the vehicle profiles can be associated with exhaust emissions, deposited tailpipe emissions and the abrasion of tar-sealed surfaces. Iron and copper are typically present in brake wear dust (Thorpe and Harrison 2008).

The third factor was characterized as marine aerosol because of high $\mathrm{Cl}$ concentrations. The marine aerosol contribution to $\mathrm{PM}_{10}$ concentrations was $1,2,1$ and $2 \%$ at NIS, NCC, NFS and Aloft, respectively. Marine aerosol is a common component in $\mathrm{PM}_{10}$ throughout New Zealand. The fourth factor was characterized as residual oil combustion based on the presence of $\mathrm{S}$ and $\mathrm{V}$ in the source profile (Qin et al. 2006; Kim and Hopke 2008; Ault et al. 2009). This source contribution likely resulted from shipping traffic at the Port of Nelson (discussed further in Chapter 10.4.3), and was therefore termed shipping sulfate. Shipping sulfate contributed 1,3 , 4 and $4 \%$ to $\mathrm{PM}_{10}$ concentrations at NIS, NCC, NFS and Aloft, respectively. The low shipping sulfate contribution at NIS is not surprising, since it was the furthest away from the port. Prior to this study, shipping had not been identified as a potential source of $\mathrm{PM}_{10}$, and is therefore never accounted for in local emission inventories. These results indicate that emissions from ship traffic at the port constitute a significant portion of measured $\mathrm{PM}_{10}$ and should be accounted for in future emissions inventories. 
The fifth factor was identified as crustal matter based on the presence of $\mathrm{Al}$, $\mathrm{Si}, \mathrm{K}, \mathrm{Ca}$ and $\mathrm{Fe}$ in the source profile. Crustal matter accounted for 3, 5, 1 and 1\% of the $\mathrm{PM}_{10}$ mass at NIS, NCC, NFS and Aloft, respectively.

The average hourly source contributions at each site were calculated to assess variations in source contributions on an hourly time-scale. Figure 10.10 presents the average hourly source contributions at NCC and Figures 10.11, 10.12 and 10.13 present the average hourly source contributions at NIS, NFS and Aloft, respectively.

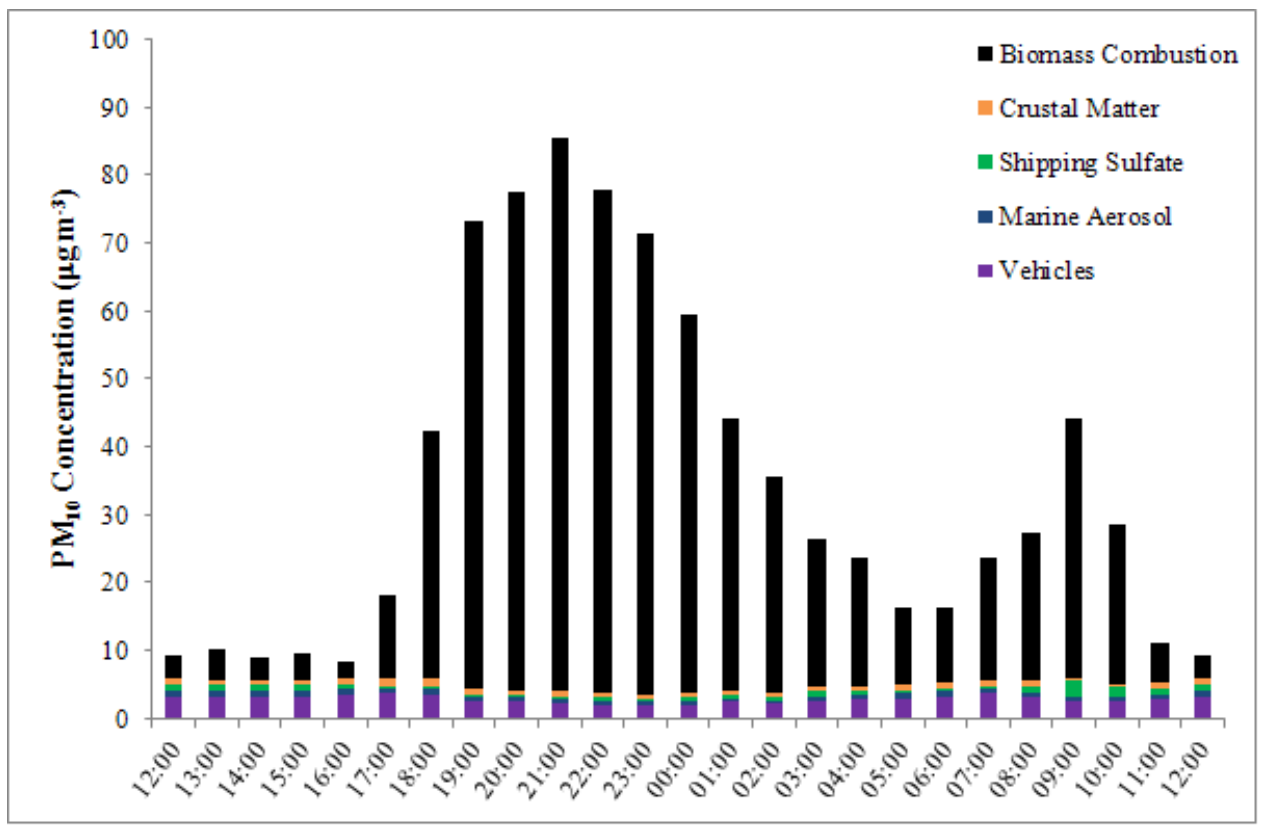

Figure 10.10 Average hourly source contributions at the Nelson City Council site.

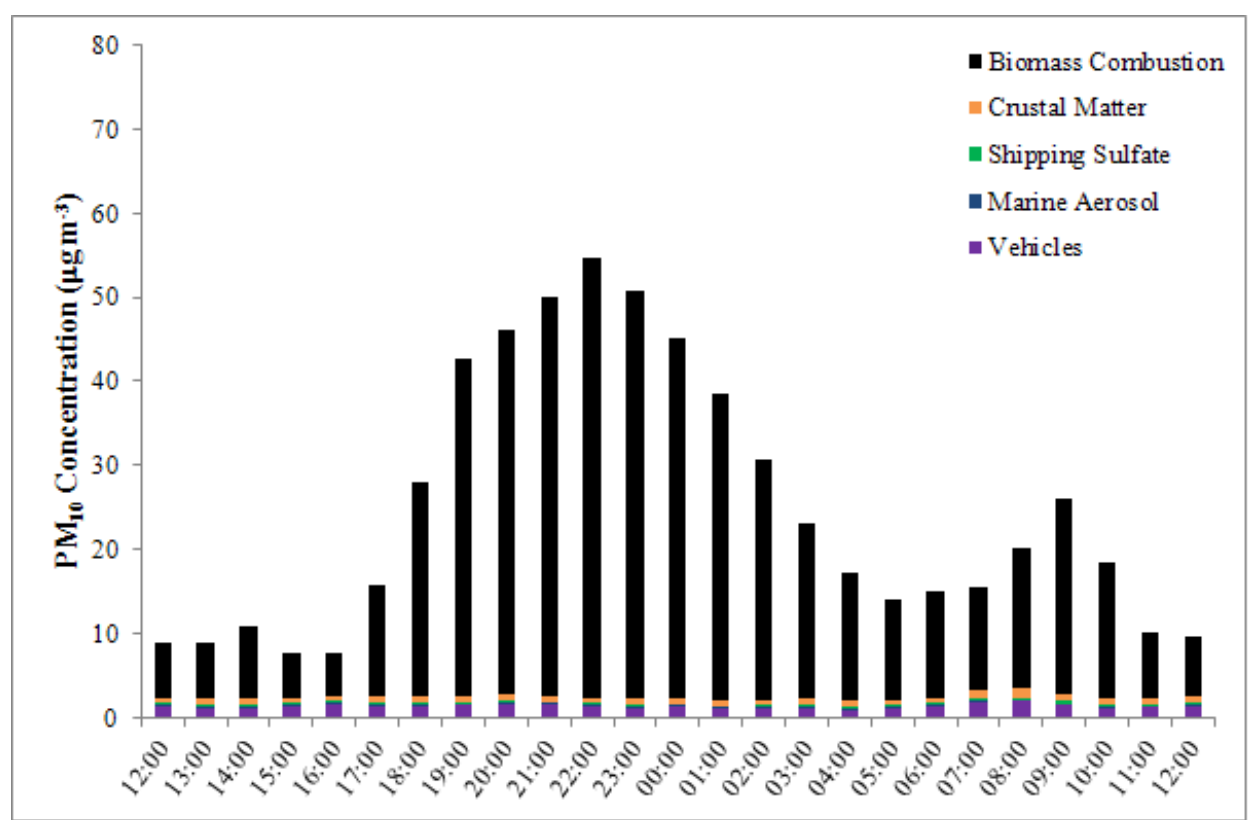

Figure 10.11 Average hourly source contributions at the Nelson Intermediate School site. 


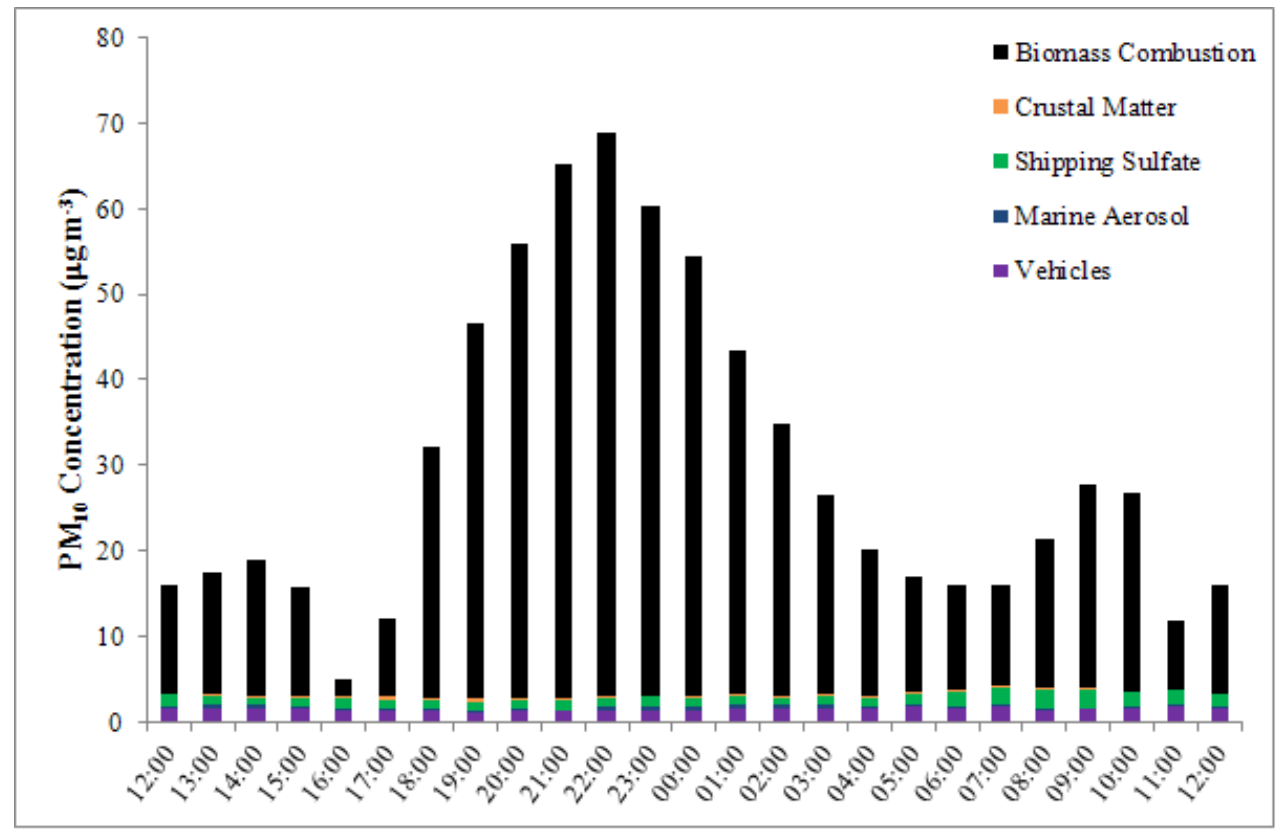

Figure 10.12 Average hourly source contributions at the Nelson Fire Station site.

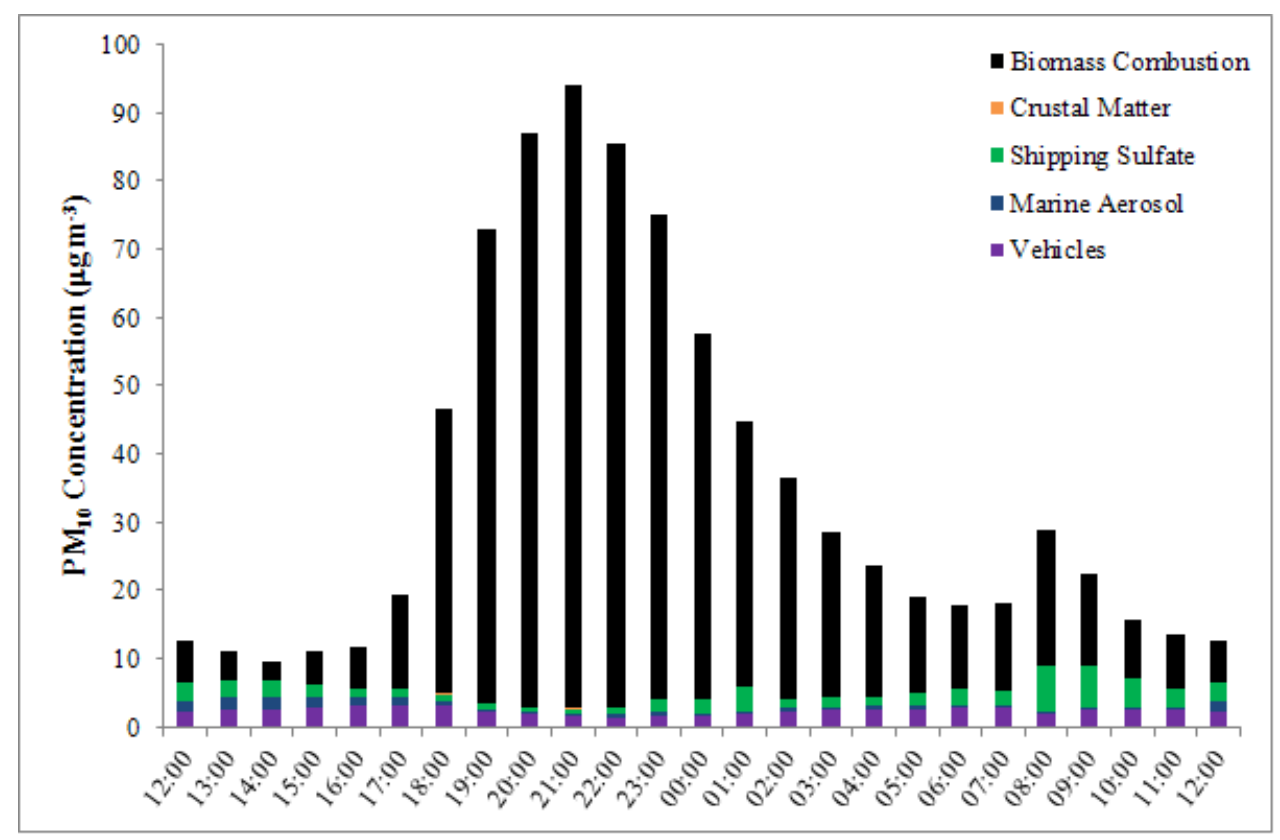

Figure 10.13 Average hourly source contributions at the Aloft site.

A number of notable features are apparent from Figures 10.10, 10.11, 10.12 and 10.13. First, biomass combustion is a significant PM source almost every hour during the winter. Biomass combustion was responsible for both peaks (evening and morning) observed in the $\mathrm{PM}_{10}$ diurnal cycle. It is not surprising that biomass combustion dominated the evening peak, since on cold winter evenings home heating is necessary and in Nelson, many households are reliant on wood burners as their main heating source. Interestingly, biomass combustion also dominated the morning 
PM peak at each of the sites. This phenomenon was also observed in Masterton, New Zealand (Ancelet et al. 2012b), and suggests that Nelson residents are relighting their fires when they rise in the morning. It was suggested by Trompetter et al. (2010) that the morning peak could also result from built-up $\mathrm{PM}_{10}$ above the inversion layer being re-entrained to ground level by atmospheric mixing upon the break-up of the inversion, which has been reported previously (Aryal et al. 2009). Based on measurements from the Aloft site and ground observations (clear visibility during the early morning), this mechanism was ruled out. We therefore suggest that katabatic flows result in dispersion and a consequent decrease in $\mathrm{PM}_{10}$ concentrations during the early morning when there are fewer new particle emissions from biomass combustion. The morning peak then arises from fires that are lit or re-stoked in the morning.

Vehicle contributions at each of the sites increased during peak traffic hours. Marine aerosol and crustal matter contributions were generally highest when hourly wind speeds were high. Shipping sulfate contributions decreased during the evening and early morning, probably because of limited up-valley transport. Large increases in shipping sulfate contributions were apparent at the Aloft site during a number of hours and we suggest this was the result of the Aloft site being more exposed to shipping plumes than the ground level sites.

\subsubsection{Source Transport}

Polar plots using the hourly source contributions were prepared to further investigate the transport mechanism and potential source locations (Carslaw and Ropkins 2011; R Development Core Team 2011). Using polar plots, the source contributions can be plotted as a function of both wind speed and direction, making them more effective than pollution roses that can only indicate directionality. To provide insight into the observed diurnal variations in source contributions, the hourly source contribution data were divided into night (6 pm-8 am) and day (9 am-5 pm). The source contributions (in $\mu \mathrm{g} \mathrm{m}^{-3}$ ) shown on the polar plots should not be taken as actual concentrations. The values actually indicate the average concentration for each wind speed/direction bin.

Figure 10.14 (a) and (b) presents biomass combustion polar plots obtained using data from the night and day, respectively, at the NCC site. Figure 10.14 
indicates that biomass combustion contributions were highest under low wind speeds and southwesterly winds, consistent with the suggestion in Chapter 10.4.1 that katabatic flows northeast along the downslope contour of the valley were responsible for the elevated $\mathrm{PM}_{10}$ concentrations observed at the NCC site.

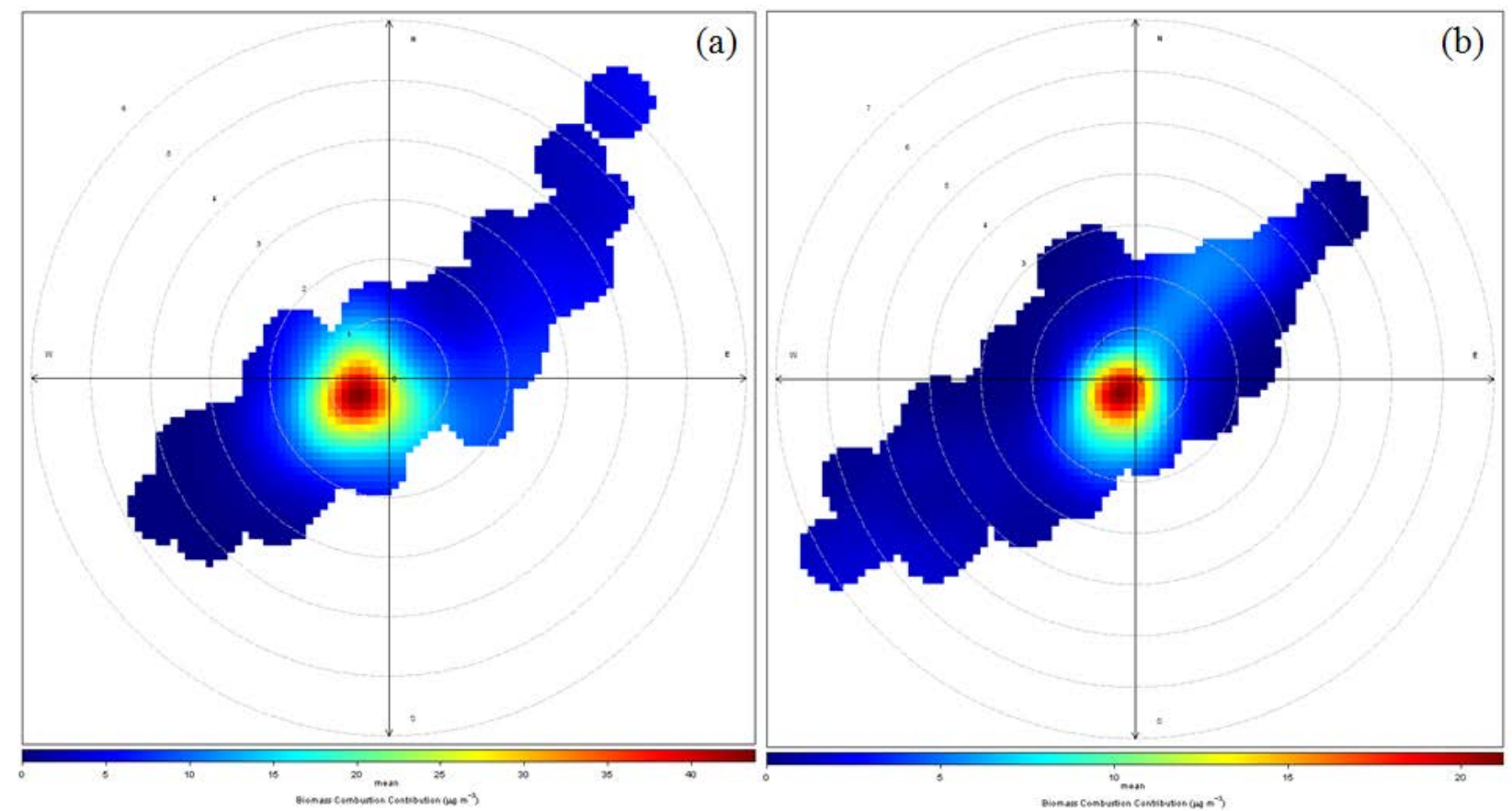

Figure 10.14 Polar plots of biomass combustion contributions during the night (a) and day (b) at the NCC site. The radial dimensions indicate the wind speed in $1 \mathrm{~ms}^{-1}$ increments and the color contours indicate the average contribution to each wind direction/speed bin.

Contributions from vehicular sources at each of the sites increased during peak traffic hours, but vehicle contributions were also apparent during hours when traffic flows would be expected to be minimal. Since Nelson is a small city, it is likely that vehicle traffic (heavy-duty diesel vehicles in particular) provides a consistent $\mathrm{PM}_{10}$ source throughout the day. Figure 10.15 (a) and (b) presents polar plots of vehicle contributions during the night and day, respectively. During the night, vehicle contributions at NCC were transported to the site by the predominantly southwesterly flows experienced within the valley. During the day (Figure 10.15 (b)), vehicle contributions were largely from the southwest and high contributions from the city center located northeast of the site were also apparent. 


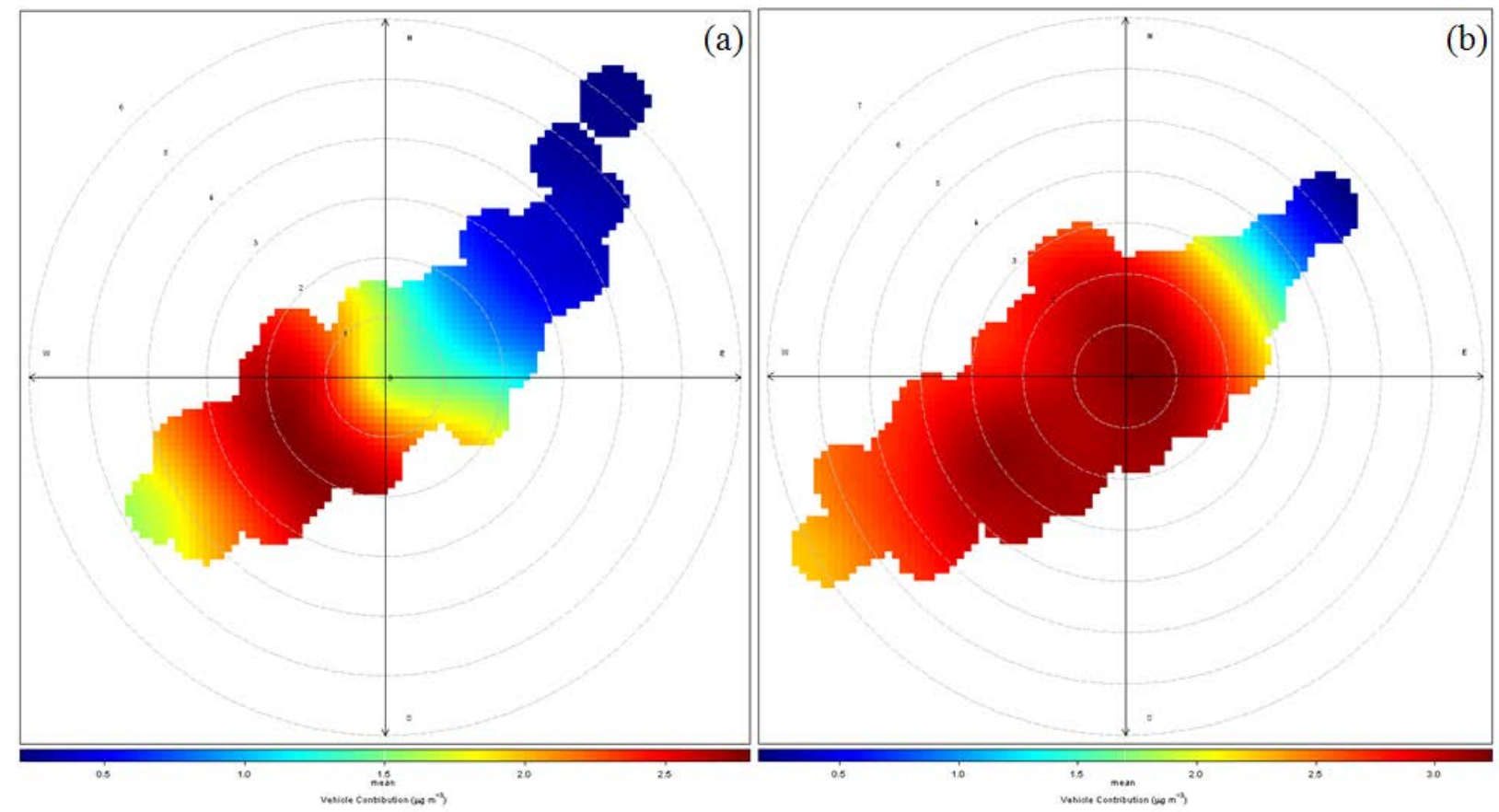

Figure 10.15 Polar plots of vehicle contributions during the night (a) and day (b) at the NCC site.

The radial dimensions indicate the wind speed in $1 \mathrm{~ms}^{-1}$ increments and the color contours indicate the average contribution to each wind direction/speed bin.

Marine aerosol contributions were more pronounced when hourly wind speeds were highest. It is well-known that marine aerosol concentrations increase under increased wind speeds (Fitzgerald 1991). Night and day polar plots of marine aerosol contributions at NCC were in agreement with this and are presented in Figure 10.16 (a) and (b). Figure 10.16 indicates that marine aerosol contributions were highest under elevated wind speeds from the northeast, in the direction of the Pacific Ocean. 


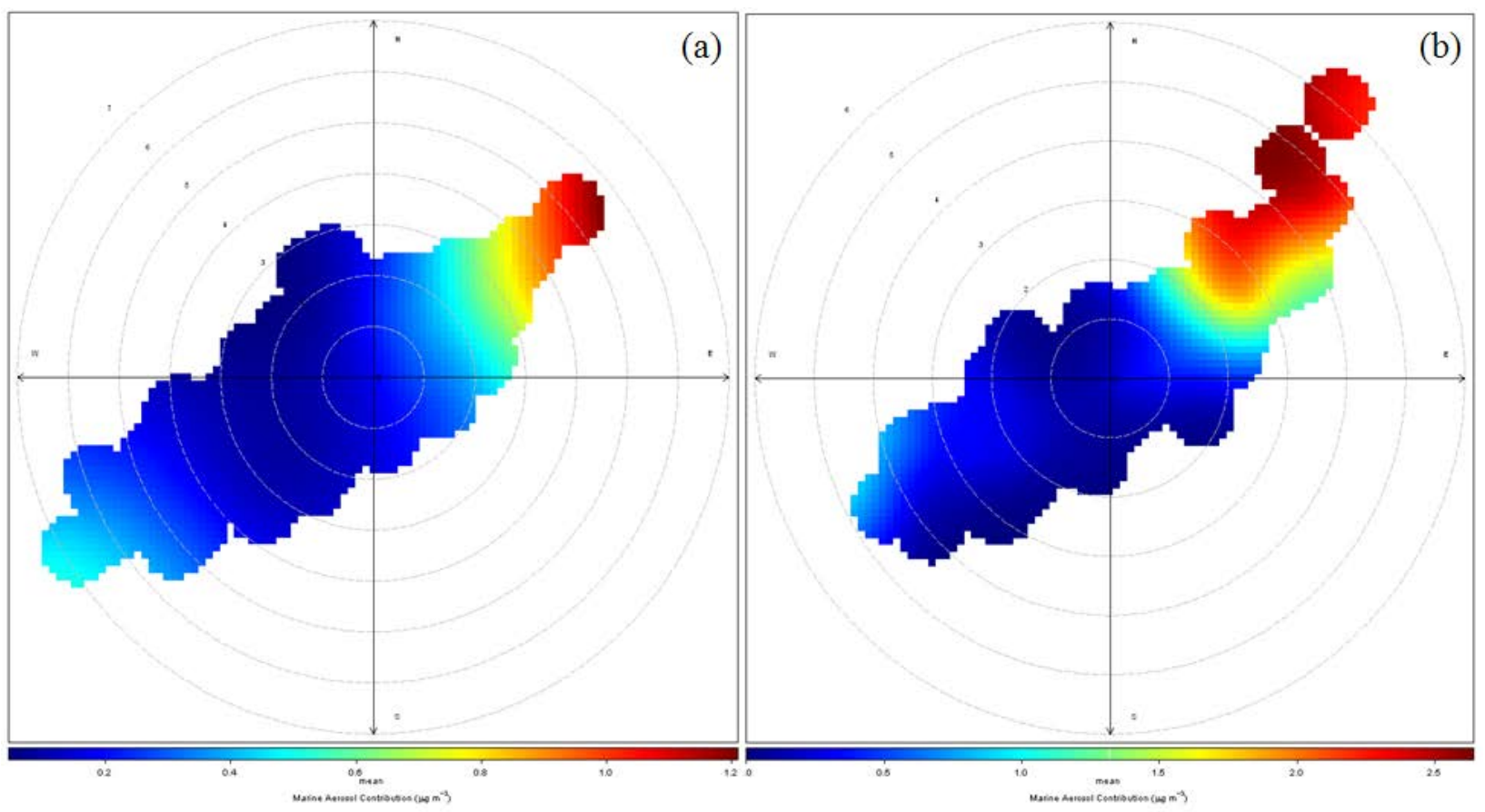

Figure 10.16 Polar plots of marine aerosol contributions during the night (a) and day (b) at the NCC site. The radial dimensions indicate the wind speed in $1 \mathrm{~ms}^{-1}$ increments and the color contours indicate the average contribution to each wind direction/speed bin.

Figure 10.17 (a) and (b) present night and day polar plots, respectively, of shipping sulfate contributions. The polar plots confirm that this source is the result of shipping emissions and not another residual oil combustion source because shipping sulfate contributions during the day and night were the result of transport from the Port of Nelson area. 


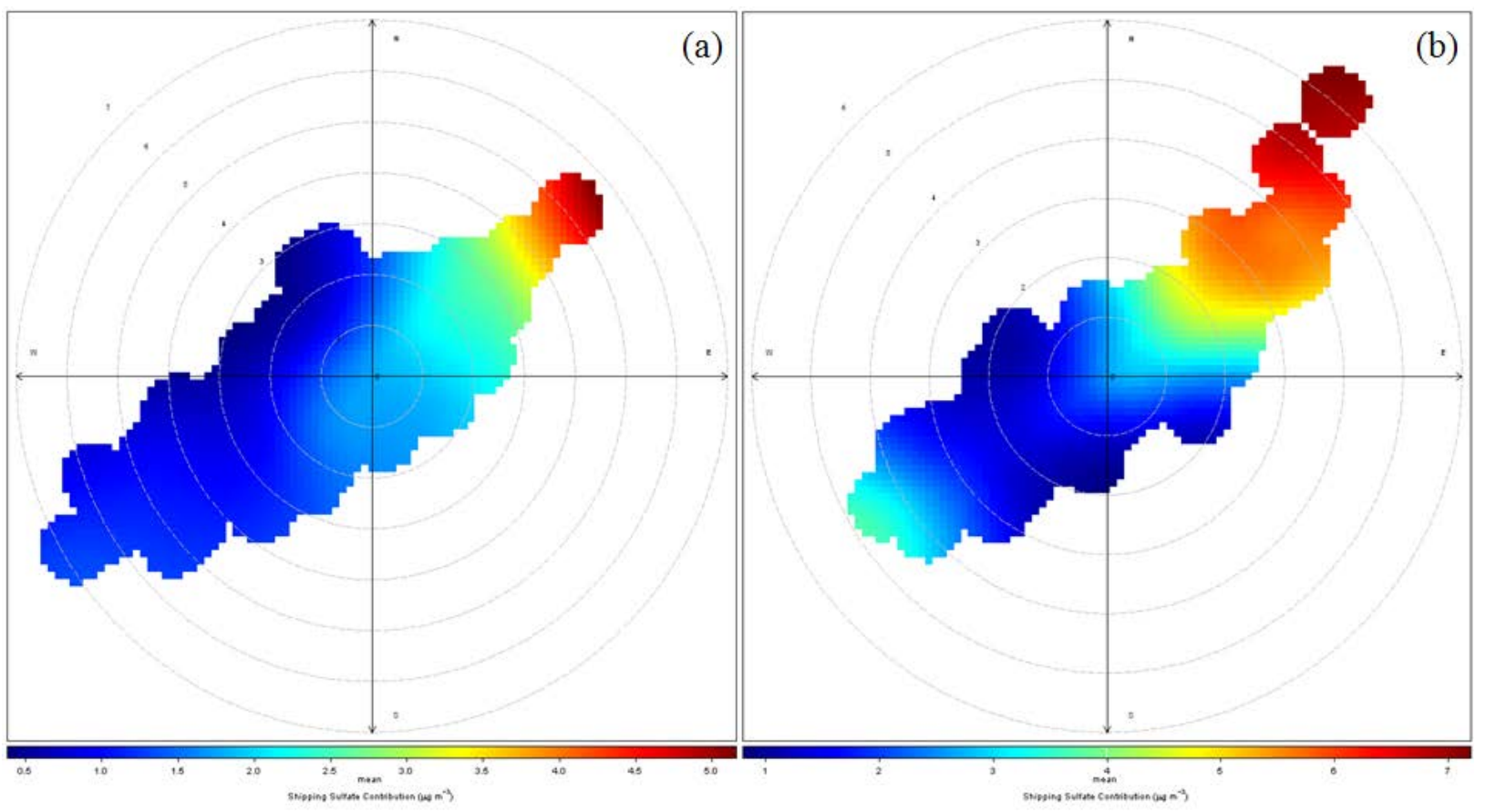

Figure 10.17 Polar plots of shipping sulfate contributions during the night (a) and day (b) at the NCC site. The radial dimensions indicate the wind speed in $1 \mathrm{~ms}^{-1}$ increments and the color contours indicate the average contribution to each wind direction/speed bin.

Night and day polar plots of crustal matter contributions at NCC are presented in Figure 10.18 (a) and (b), respectively. Crustal matter contributions were greatest under high wind speeds from the southwest. Low wind speed crustal matter contributions from the north, particularly during the day, were likely from land adjacent to NCC where construction activities were taking place. 


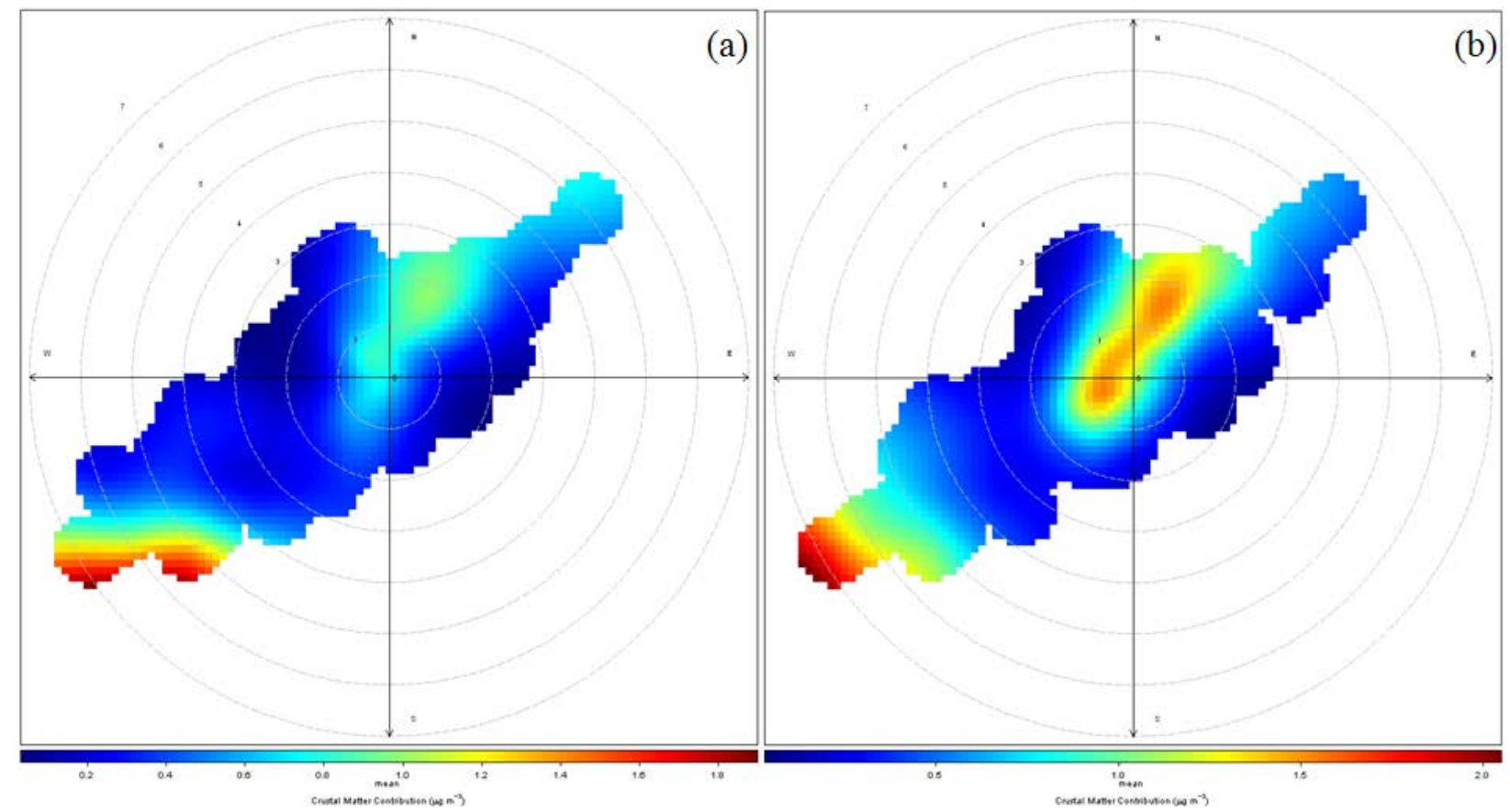

Figure 10.18 Polar plots of crustal matter contributions during the night (a) and day (b) at the NCC site. The radial dimensions indicate the wind speed in $1 \mathrm{~ms}^{-1}$ increments and the color contours indicate the average contribution to each wind direction/speed bin.

\subsection{Acknowledgments}

The support of Nelson City Council (Paul Sheldon) was greatly appreciated. We thank Bruce Crothers and Ed Hutchinson for their support in maintaining the sampling equipment, Chris Purcell for setting up and maintaining the $3 \mathrm{MeV}$ accelerator used for IBA and Stuart Grange for his support with the sampling. This work was funded by the Ministry of Science and Innovation under contract C05X0903. 


\section{Alexandra: Particulate matter sources on an hourly time-scale in a rural community during the winter}

\subsection{Synopsis}

This chapter has been reproduced in full, as prepared for submission to Environmental Science and Technology, 2012. Ancelet, T., Davy, P. K., Trompetter, W. J., Markwitz, A. and Weatherburn, D. C., 2012. Particulate matter sources on an hourly time-scale in a rural community during the winter. Environmental Science and Technology, in preparation.

This study was performed in Alexandra, a small community located in an inland sub-alpine basin on New Zealand's South Island. The same experimental design that was used in Nelson (described in Chapter 10) was used, with three sites located on a horizontal transect across the town and a fourth site located centrally, but at a height of $26 \mathrm{~m}$. While $\mathrm{PM}_{10}$ concentrations in Masterton and Nelson were shown to display similar diurnal patterns, regardless of location, average hourly $\mathrm{PM}_{10}$ concentrations in Alexandra showed slightly different diurnal profiles depending on the sampling site location. Each location did, however, feature a large evening peak and smaller morning peak in $\mathrm{PM}_{10}$ concentrations. The central site in Alexandra experienced the highest $\mathrm{PM}_{10}$ concentrations and the convergence of numerous katabatic flows was identified as the principal reason for this. A significant difference in $\mathrm{PM}_{10}$ concentrations between the central and elevated sites indicated that a shallow inversion layer formed below the elevated site, limiting the vertical dispersion of pollutants.

Four $\mathrm{PM}_{10}$ sources were identified at each of the sites: biomass combustion, vehicles, crustal matter and marine aerosol. Biomass combustion was identified as the most significant source of $\mathrm{PM}_{10}$, contributing up to $91 \%$ of the measured $\mathrm{PM}_{10}$. Plots of the average hourly source contributions to each site revealed that biomass combustion was responsible for both the evening and morning peaks in $\mathrm{PM}_{10}$ concentrations observed at each of the sites. This suggests that Alexandra residents are re-lighting their fires when they rise in the morning. 


\subsection{Introduction}

Particulate matter (PM) is well-known to have adverse effects on human health and a range of environmental effects, including local reductions in visibility and effects on the Earth's radiative balance (Dockery et al. 1993; Tsai and Cheng 1999; Nel 2005; Russell and Brunekreef 2009; Pósfai and Buseck 2010). Therefore, the benefits of understanding the sources and factors contributing to elevated PM concentrations are direct and tangible. In New Zealand, PM concentrations have been shown to have distinct diurnal cycles, with peak concentrations occurring during the evening and morning (Trompetter et al. 2010). Currently, very little is known about the sources and factors responsible for the observed diurnal cycles.

We have recently reported the first PM source apportionment study using hourly data obtained from two sampling sites in the rural community of Masterton, New Zealand (Ancelet et al. 2012b). In that study, biomass combustion was identified to be the dominant PM source during both the evening and the morning. To identify whether this situation was unique to Masterton, an intensive high-temporal resolution monitoring campaign was undertaken in Alexandra, New Zealand (latitude $-45.16^{\circ}$, longitude $169.22^{\circ}$ ) during the 2011 winter. Alexandra is a rural town located in an inland basin with a population of approximately 4 800. Despite its small population, Alexandra suffers from poor air quality during the winter when domestic combustion for home heating and strong temperature inversions that limit the dispersion of pollutants are common.

In this study, positive matrix factorization (PMF) was used to identify the major $\mathrm{PM}_{10}(\mathrm{PM}<10 \mu \mathrm{m}$ in aerodynamic diameter) sources at four sites in Alexandra on an hourly time-scale. PMF is a powerful and commonly used multivariate method capable of resolving factors contributing to PM samples without prior source knowledge and the results of the analysis are directly interpretable as mass contributions from each factor (source) (Paatero and Tapper 1994; Paatero 1997; Song et al. 2001). Two receptor models are available to perform PMF, PMF2 (Paatero 1997) and EPA PMF (USEPA 2008). Despite some differences in model operation (Paatero 1999), PMF2 and EPA PMF have been shown to provide similar results with some minor differences in the final solutions (Kim and Hopke 2007).

Using hourly source contributions and meteorological data from each of the sites, potential PM transport mechanisms were also identified. High-temporal 
resolution source apportionment studies can provide unique and highly relevant information for the implementation of PM mitigation strategies.

\subsection{Experimental Section}

\subsubsection{Sample Collection}

Ambient air monitoring was conducted at four locations in Alexandra. Three of the sites were located along the general katabatic flow pathway (upwind, central and downwind) (Manins and Sawford 1979). The fourth site (Aloft) was also located centrally, but was raised to a height of $26 \mathrm{~m}$ above ground level using a knuckleboom when wind conditions permitted $\left(<3 \mathrm{~m} \mathrm{~s}^{-1}\right)$. Under higher wind speeds, the knuckleboom was kept at ground level. We have recently reported this type of experimental setup and Figure 11.1 presents a schematic illustration of the sampling site locations (Ancelet et al. 2012a). The sampling site locations were designed to provide an indication of PM transport horizontally and vertically within the Alexandra airshed.

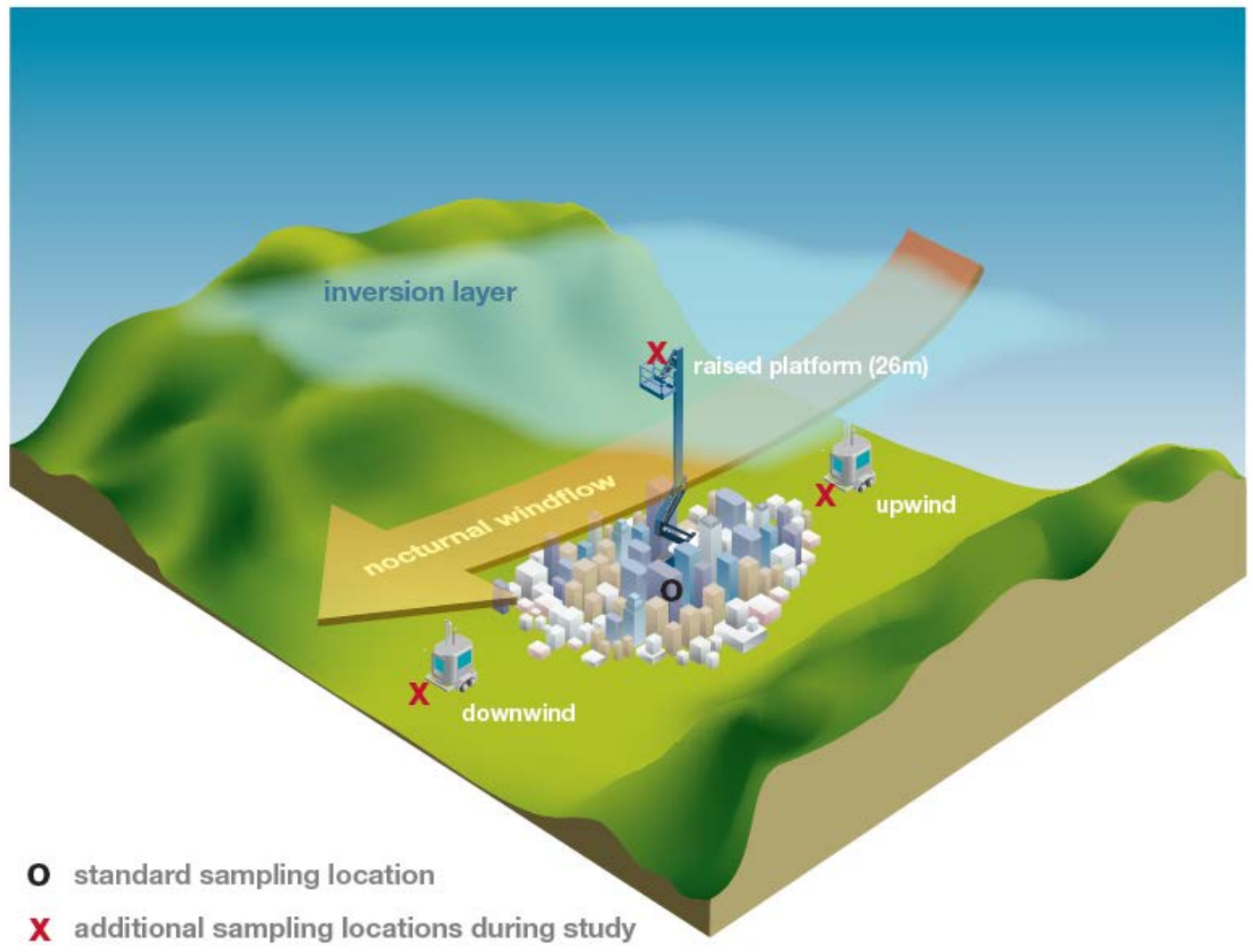

Figure 11.1 Schematic illustration of the sampling site locations in Alexandra. 
The upwind site was located on the grounds of the Alexandra Bowling Club (ABC) (latitude $-45.143957^{\circ}$, longitude $169.225102^{\circ}$ ). The ABC site was situated approximately $600 \mathrm{~m}$ northwest of the central site and $70 \mathrm{~m}$ from the nearest road. The central and elevated sites were located at the Alexandra Girl Guides center (GG) (latitude $-45.145753^{\circ}$, latitude $169.230385^{\circ}$ ) and were approximately $50 \mathrm{~m}$ from the nearest road. The GG site was co-located with an ambient air quality monitoring station used for compliance monitoring by the Otago Regional Council. The downwind site was located in an open area next to the offices of the Central Otago District Council (CODC) (latitude $-45.152455^{\circ}$, longitude $169.232152^{\circ}$ ). The CODC site was approximately $1 \mathrm{~km}$ southeast of the GG site and $100 \mathrm{~m}$ from the Clutha River. The land around each of the sites was flat and surrounded by open space or buildings no more than two stories high. Figure 11.2 presents the locations of each of the sampling sites.

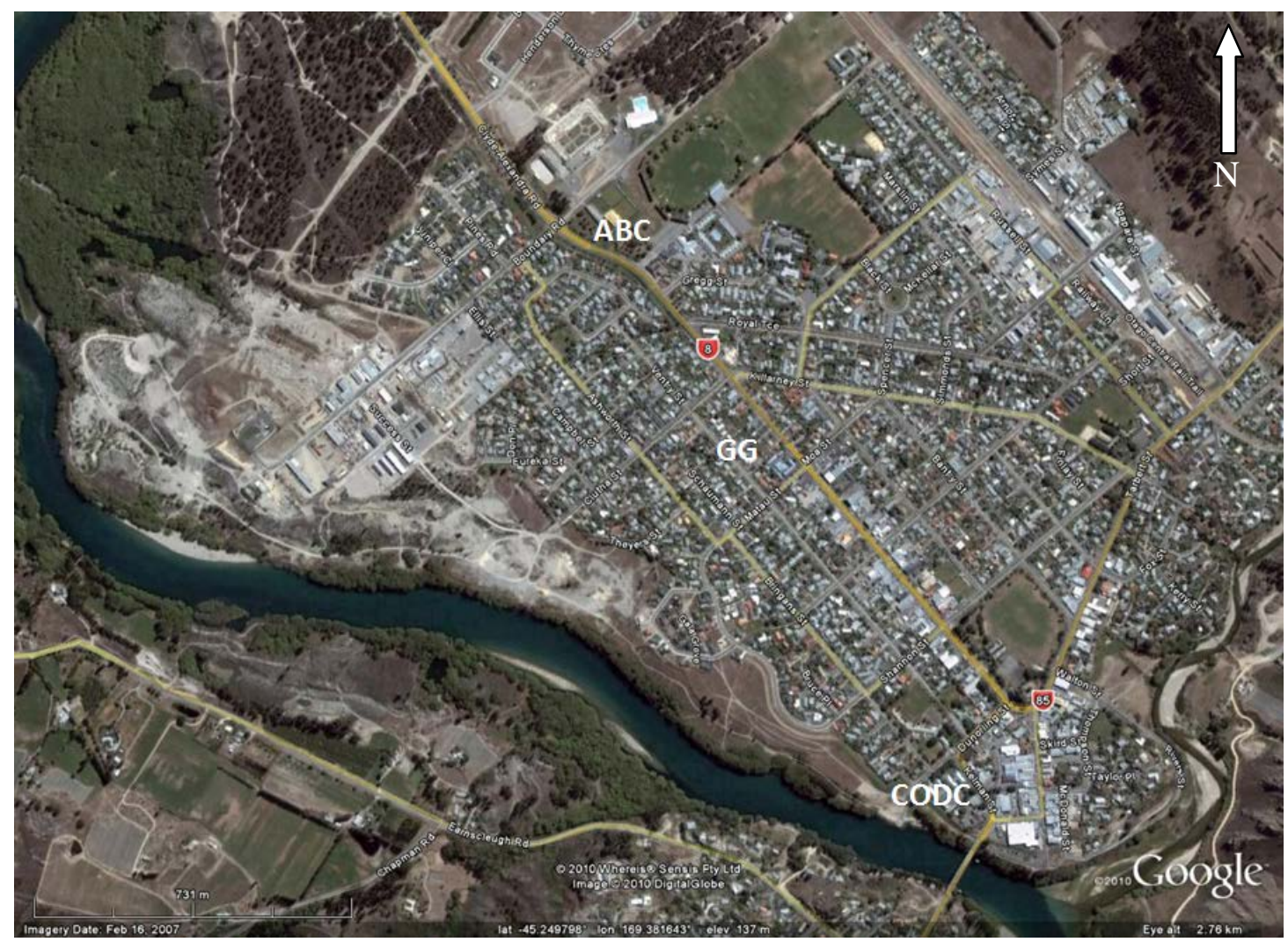

Figure 11.2 Locations of the sampling sites within Alexandra (ABC: Alexandra bowling club; GG: Girl Guides center; CODC: Central Otago District Council offices). The Aloft site was co-located with the GG site. The Clutha River flows from left to right in this figure. 
Each site was equipped with a Streaker sampler (PIXE International Corporation, USA), an E-BAM (Met One Instruments, Inc.) and a meteorological station (Vaisala WXT520) in the same fashion as previously reported (Ancelet et al. 2012b). In this study, a total of 47 samples, or 47 hours, were collected on each set of size-resolved ( $\mathrm{PM}_{10-2.5}$ and $\left.\mathrm{PM}_{2.5}\right)$ filters. The monitoring program ran from AprilJune 2011 and a total of 7406 samples were collected among the four sites (1784 from ABC, 2054 from GG, 1880 from CODC and 1690 (522 when raised to $26 \mathrm{~m}$ ) from the Aloft site).

\subsubsection{Elemental Analysis}

Ion beam analysis (IBA) was used to measure the concentrations of elements with atomic numbers above neon in the PM collected. IBA measurements were carried out at the New Zealand Ion Beam Analysis Facility operated by the Institute of Geological and Nuclear Sciences (GNS) in Gracefield, Lower Hutt, New Zealand (Trompetter et al. 2005). Further details on the IBA techniques used, analytical uncertainties and limits of detection have been reported previously (Ancelet et al. 2012b). Black carbon was measured using a M43D Digital Smoke Stain Reflectometer (Ancelet et al. 2011b). Prior to the PMF analyses, data and uncertainty matrices were prepared for each site in the same manner as previous studies (Polissar et al. 1998; Song et al. 2001).

\subsubsection{Receptor Modeling}

Receptor modeling and apportionment of PM mass by PMF was performed using the EPAPMF version 3.0.2.2 program in accordance with the User's Guide (11). With PMF, sources are constrained to have non-negative species concentrations, no sample can have a negative source contribution and error estimates for each observed point are used as point-by-point weights. This is a distinct advantage of PMF, since it can accommodate missing or below detection limit data that is a common feature of environmental monitoring (Song et al. 2001). Data screening and the source apportionment were performed in the same manner as previously reported (Ancelet et al. 2012b). Table 11.1 provides a summary of the parameters used for the PMF analyses and the diagnostics obtained for each of the sites. 
Table 11.1 Parameters used for PMF analyses and diagnostics obtained from the analyses for each of the sites

\begin{tabular}{|c|c|c|c|c|}
\hline & $\begin{array}{c}\text { Alexandra Bowling } \\
\text { Club }\end{array}$ & Girl Guides Center & $\begin{array}{c}\text { Central Otago } \\
\text { District Council } \\
\text { offices } \\
\end{array}$ & Aloft \\
\hline $\begin{array}{l}\text { Extra modeling } \\
\text { uncertainty (\%) }\end{array}$ & 5 & 5 & 5 & 5 \\
\hline $\begin{array}{c}\text { Number of base } \\
\text { runs }\end{array}$ & 20 & 20 & 20 & 20 \\
\hline Base run seed & Random & Random & Random & Random \\
\hline $\begin{array}{l}\text { Number of } \\
\text { bootstraps }\end{array}$ & 200 & 200 & 200 & 200 \\
\hline $\begin{array}{c}\text { Minimum } \\
\text { correlation R-value }\end{array}$ & 0.6 & 0.6 & 0.6 & 0.6 \\
\hline Bootstrap seed & Random & Random & Random & Random \\
\hline Block size & 24 & 24 & 24 & 24 \\
\hline Theoretical Q & 4460 & 8657 & 5953 & 4225 \\
\hline Robust Q & 3634 & 7000 & 3231 & 2011 \\
\hline True Q & 3853 & 7446 & 3294 & 2101 \\
\hline $\begin{array}{c}\text { Number of } \\
\text { bootstrapped factors } \\
\text { mapped to original } \\
\text { factor } 1 \\
\end{array}$ & 200 & 199 & 200 & 200 \\
\hline $\begin{array}{c}\text { Number of } \\
\text { bootstrapped factors } \\
\text { mapped to original } \\
\text { factor } 2\end{array}$ & 200 & 200 & 200 & 200 \\
\hline $\begin{array}{c}\text { Number of } \\
\text { bootstrapped factors } \\
\text { mapped to original } \\
\text { factor } 3 \\
\end{array}$ & 200 & 200 & 200 & 191 \\
\hline $\begin{array}{c}\text { Number of } \\
\text { bootstrapped factors } \\
\text { mapped to original } \\
\text { factor } 4 \\
\end{array}$ & 194 & 200 & 200 & 200 \\
\hline $\begin{array}{c}\text { Number of } \\
\text { bootstrapped factors } \\
\text { mapped to no } \\
\text { original factor }\end{array}$ & 5 & 1 & 0 & 7 \\
\hline Maximum D value & $<0.1$ & $<0.1$ & $<0.1$ & $<0.1$ \\
\hline
\end{tabular}

\subsection{Results and Discussion}

\subsection{1 $\mathrm{PM}_{10}$ Concentrations}

$\mathrm{PM}_{10}$ concentrations at all four of the sites displayed distinct diurnal cycles. Figure 11.3 (a) presents the average hourly $\mathrm{PM}_{10}$ concentrations at the $\mathrm{ABC}, \mathrm{GG}$ and CODC sites over the entire sampling period. From Figure 11.3 (a) it is apparent that measured $\mathrm{PM}_{10}$ concentrations in Alexandra are strongly dependent on the sampling location. While each of the sites had peak $\mathrm{PM}_{10}$ concentrations during the evening, the GG site generally experienced higher $\mathrm{PM}_{10}$ concentrations than the $\mathrm{ABC}$ and CODC sites and peak $\mathrm{PM}_{10}$ concentrations at the GG site (7 pm-midnight) occurred earlier than at the other sites (9 pm-2 am). This is probably because the GG site was located 
in a more densely populated area than the ABC and CODC sites, which were near the outskirts of Alexandra and had fewer immediate PM sources. A morning peak in $\mathrm{PM}_{10}$ concentrations ( $8 \mathrm{am}-10 \mathrm{am}$ ) was also apparent at each of the sites, but was much more pronounced at GG.

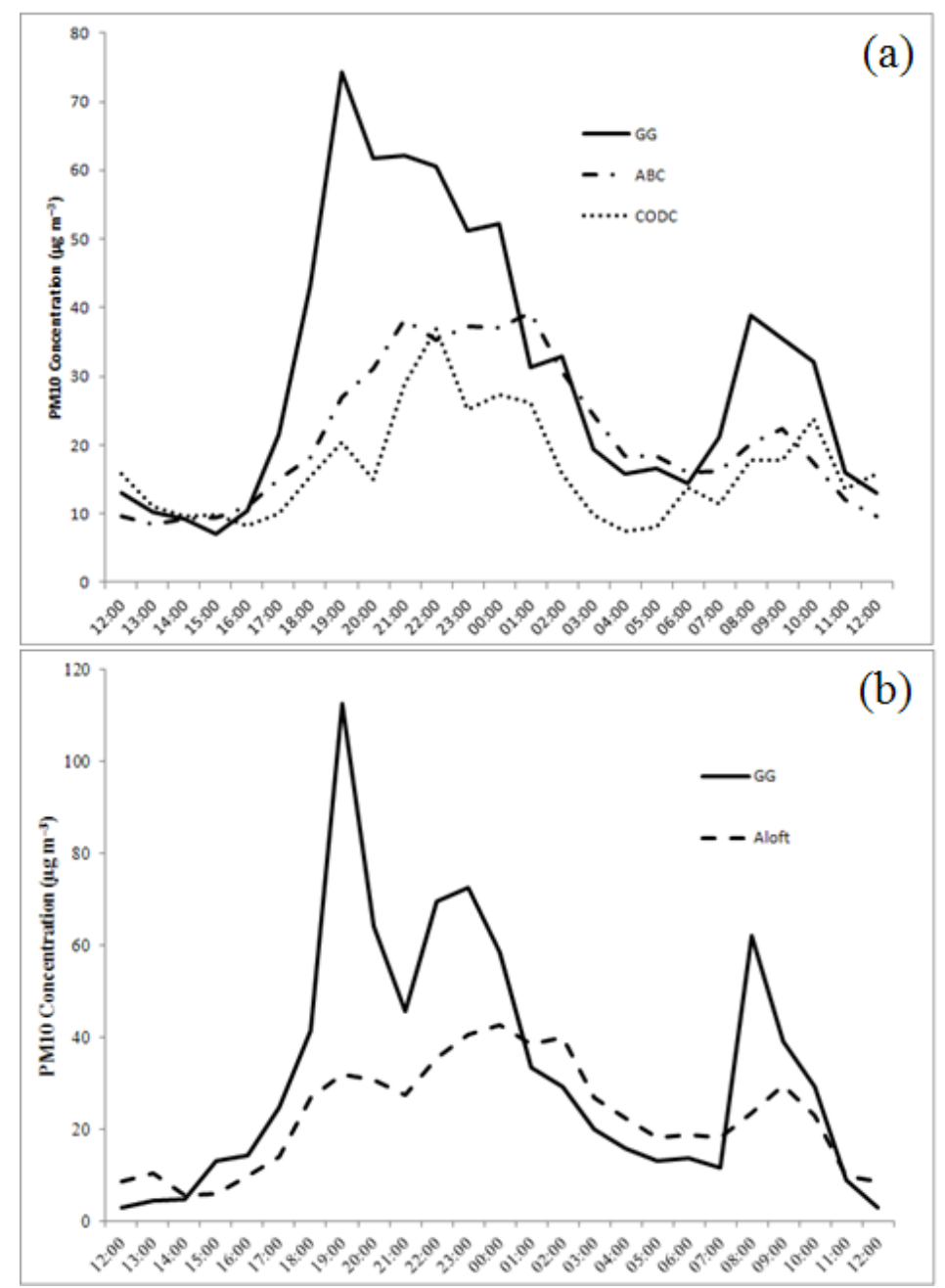

Figure 11.3 Average hourly $\mathrm{PM}_{10}$ concentrations at (a) the Bowling Club, Girl Guides and Central Otago District Council office sites and (b) at the Girl Guides and Aloft sites during periods when the knuckleboom was raised.

Figure 11.3 (b) presents average hourly $\mathrm{PM}_{10}$ concentrations at the GG and Aloft sites during periods when the knuckleboom was raised (261 hours total). Since the knuckleboom was only raised when wind speeds were low (during anticyclonic atmospheric conditions), $\mathrm{PM}_{10}$ concentrations measured at the GG site were higher than during periods when the knuckleboom was not raised because of the formation of strong temperature inversions that limited the transport of PM. Average hourly $\mathrm{PM}_{10}$ concentrations at the GG site during periods when the knuckleboom was raised 
showed a trimodal diurnal pattern, with peak $\mathrm{PM}_{10}$ concentrations occurring at $7 \mathrm{pm}$, from $10-11 \mathrm{pm}$ and at $8 \mathrm{am}$. In contrast to the ground level site, average hourly $\mathrm{PM}_{10}$ concentrations at the Aloft site (26 m height) had a bimodal diurnal pattern, with a broad evening peak (6 pm-2 am) and a morning peak at $9 \mathrm{am}$. It is also evident from Figure 11.3 (b) that average hourly $\mathrm{PM}_{10}$ concentrations at the ground level GG site were significantly higher than those aloft from 6 pm-midnight and 8-10 am, suggesting the formation of a stable inversion layer below the height of the Aloft site. Further supporting this conclusion, average wind speeds and temperatures were higher at the Aloft site than those at GG between 7-11 pm and 8-9 am, indicating that at $26 \mathrm{~m}$ the air was more well-mixed than at ground level. Between 1 and $7 \mathrm{am}$, average hourly $\mathrm{PM}_{10}$ concentrations at the Aloft site were slightly higher than at the GG site, indicating that shallow drainage winds were likely responsible for the large decrease in $\mathrm{PM}_{10}$ concentrations measured during the early morning.

Comparisons of daily average $\mathrm{PM}_{10}$ concentrations among the four sites revealed that $A B C$ and CODC did not exceed the New Zealand National Environmental Standard for $\mathrm{PM}_{10}$ of $50 \mu \mathrm{g} \mathrm{m}^{-3}$ (24-hour average) on any day during the study period, while the Aloft and GG sites had 1 and 12 exceedances, respectively, during this study. It is important to note that E-BAMs are not certified for air quality compliance monitoring, but it is clear that the sampling location can have a significant impact on measured $\mathrm{PM}_{10}$ concentrations in Alexandra.

The local meteorology in Alexandra was investigated on an hourly basis to gain a better understanding of PM transport. Figure 11.4 presents a wind rose plot over the entire sampling period from GG. Wind rose plots for ABC, CODC and Aloft were similar to that from GG. 


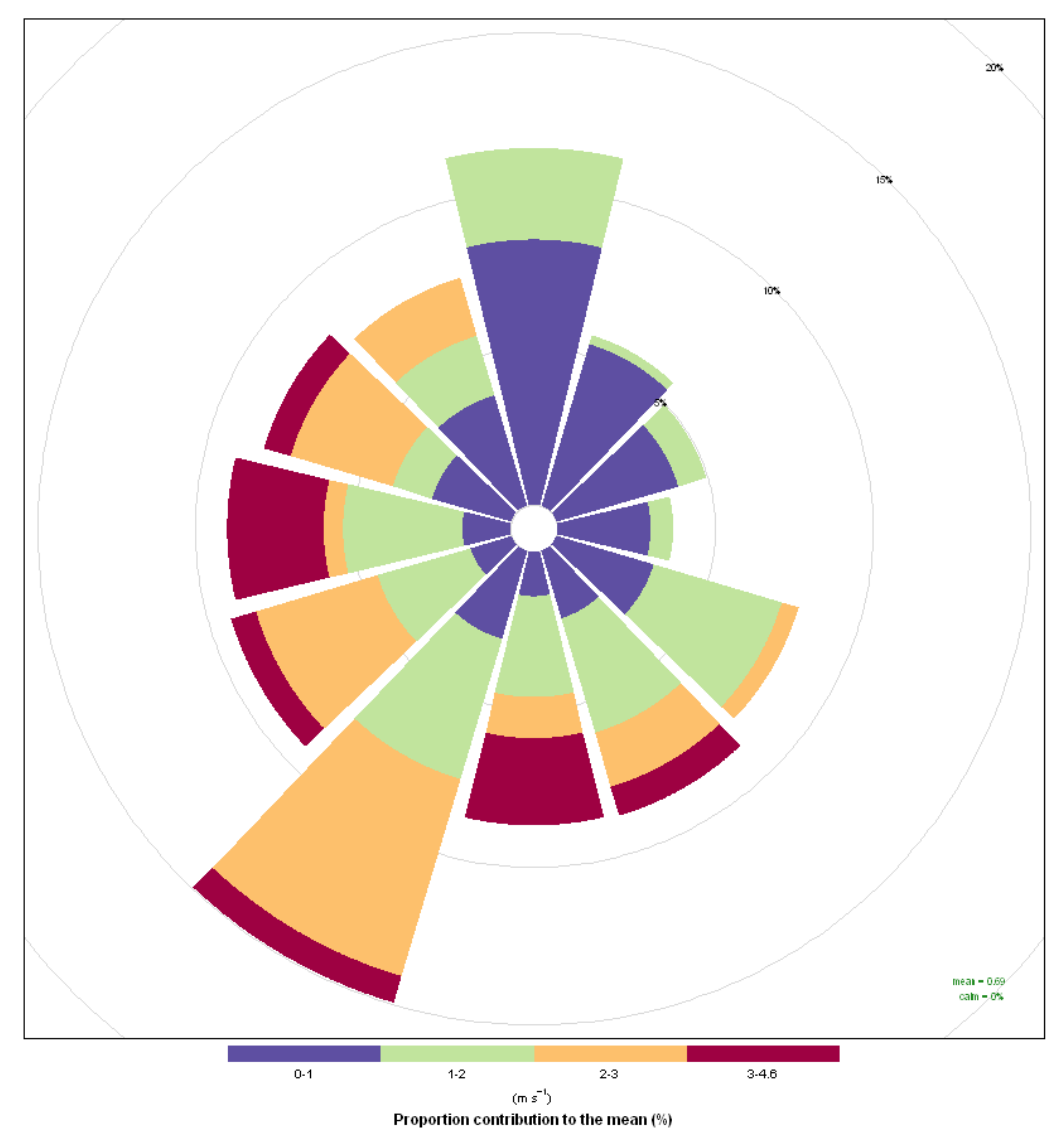

Figure 11.4 Wind rose plot over the entire sampling period from the Girl Guides site. The radial dimensions indicate the frequency (\%) of winds from each direction.

A number of features are apparent from Figure 11.4. First, winds during the sampling period were predominantly from the southwest and north, with westerly and southerly components also strongly contributing. Northerly winds were characterized by low wind speeds $\left(<2 \mathrm{~m} \mathrm{~s}^{-1}\right)$. Using the high temporal resolution data available in this study, hourly pollution roses for each of the sites were developed using the $\mathrm{R}$ statistical and Openair packages (Carslaw and Ropkins 2011; R Development Core Team 2011). Figure 11.5 presents hourly $\mathrm{PM}_{10}$ pollution roses for the GG site. Figure 11.5 suggests that the elevated $\mathrm{PM}_{10}$ concentrations measured at GG were the result of katabatic flows, generally from the north. Interestingly, the pollution roses also suggest that the GG site is situated at the convergence point of katabatic flows from different directions. Since Alexandra is located in a basin, it seems likely that PM emitted during the evening is being transported through katabatic flows to a central location along numerous downslope contours. 


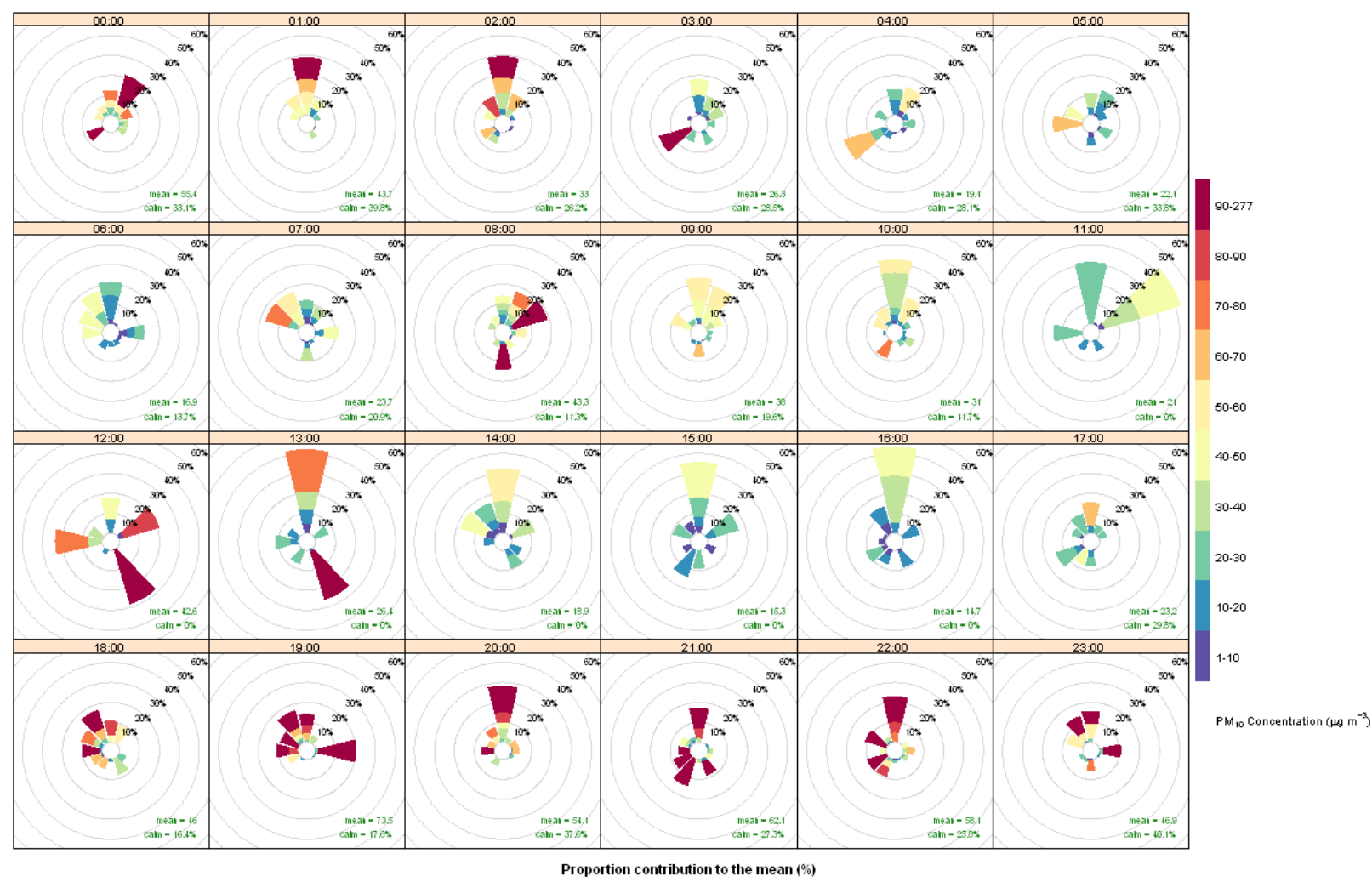

Figure 11.5 Hourly pollution roses from the Girl Guides site indicating wind directions contributing the most to average hourly $\mathrm{PM}_{10}$ concentrations. The radial dimensions indicate the percentage of the total pollution that arrives from each wind sector during each one-hour period.

In contrast to the GG site, hourly pollution roses for CODC (Figure 11.6) indicated that, during the morning and evening, $\mathrm{PM}_{10}$ was transported from the north by katabatic flows. This result demonstrates that, despite their close proximity, different areas within an airshed can be impacted by PM in different manners. 


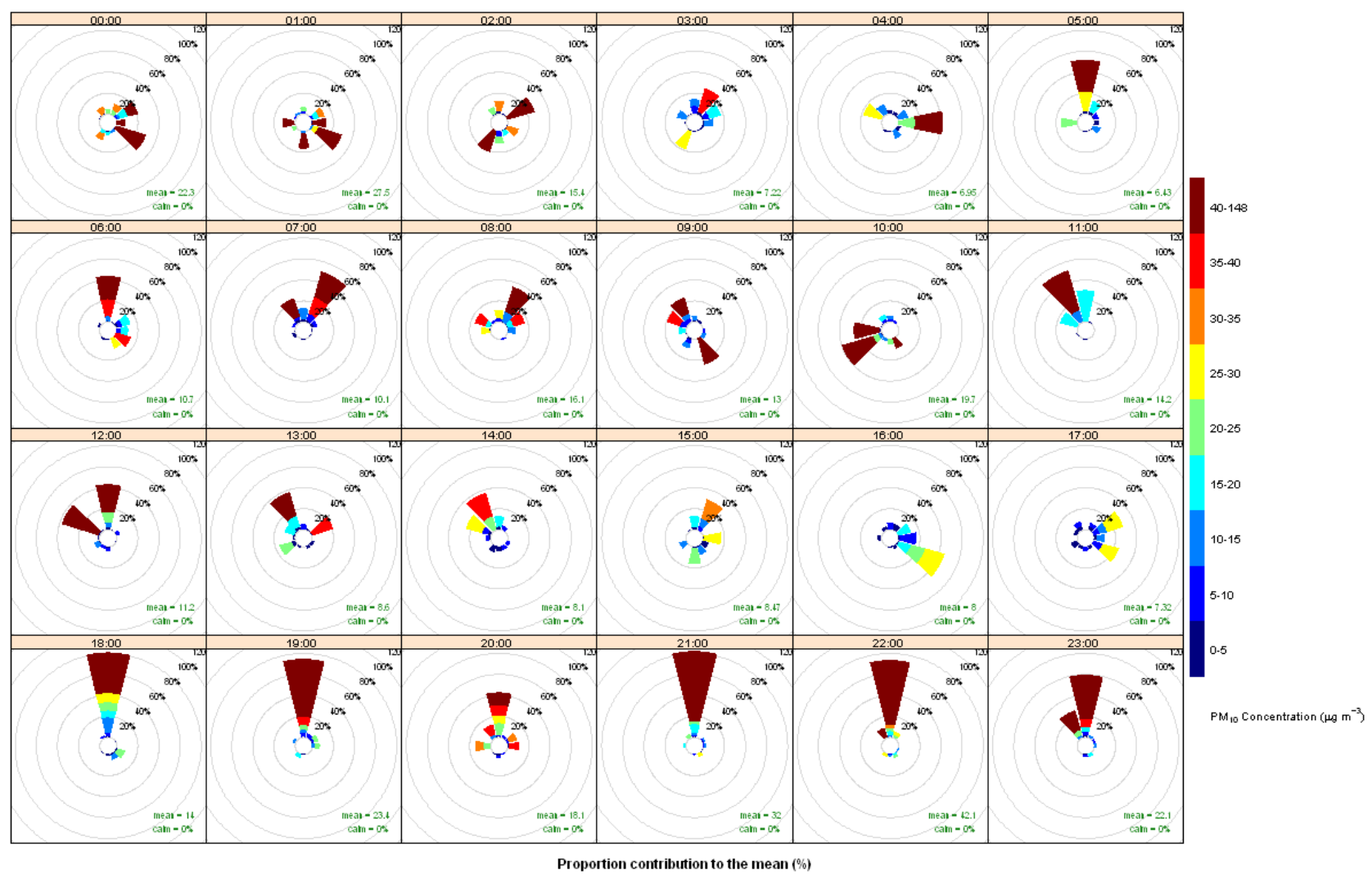

Figure 11.6 Hourly pollution roses from the Central Otago District Council site indicating wind directions contributing the most to average hourly $\mathrm{PM}_{10}$ concentrations. The radial dimensions indicate the percentage of the total pollution that arrives from each wind sector during each one-hour period.

\subsubsection{Sources of Ambient $P M_{10}$}

Tables 11.2, 11.3, 11.4 and 11.5 present the average, maximum and median hourly concentrations of species used for source apportionment at the ABC, GG, CODC and Aloft sites, respectively, along with standard deviations, average uncertainties, average limits of detection and the number of samples above the limit of detection for each species. 
Table 11.2 $\quad \mathrm{PM}_{10}\left(\mu \mathrm{g} \mathrm{m}^{-3}\right)$, BC $\left(\mathrm{ng} \mathrm{m}^{-3}\right)$ and elemental $\left(\mathrm{ng} \mathrm{m}^{-3}\right)$ data from the Alexandra Bowling Club site (892 samples)

\begin{tabular}{|c|c|c|c|c|c|c|c|}
\hline & Average & Maximum & Median & $\begin{array}{l}\text { Standard } \\
\text { Deviation }\end{array}$ & $\begin{array}{c}\text { Average } \\
\text { Uncertainty } \\
\end{array}$ & $\begin{array}{c}\text { Average } \\
\text { LOD } \\
\end{array}$ & $\begin{array}{c}\text { Number }> \\
\text { LOD }\end{array}$ \\
\hline $\mathrm{PM}_{10}$ & 19 & 120 & 11 & 21 & & & \\
\hline Fine BC & 4888 & 16202 & 4206 & 2555 & 543 & 800 & 889 \\
\hline Fine Al & 48 & 193 & 45 & 34 & 31 & 63 & 322 \\
\hline Fine Si & 180 & 1480 & 122 & 226 & 25 & 39 & 886 \\
\hline Fine S & 366 & 5753 & 120 & 891 & 23 & 26 & 892 \\
\hline Fine $\mathrm{K}$ & 109 & 1010 & 61 & 136 & 16 & 26 & 646 \\
\hline Fine Ca & 46 & 434 & 38 & 40 & 14 & 22 & 694 \\
\hline Fine $\mathrm{Fe}$ & 26 & 220 & 21 & 21 & 7 & 8 & 809 \\
\hline Fine $\mathrm{Cu}$ & 5 & 131 & 3 & 9 & 4 & 12 & 118 \\
\hline Fine $\mathrm{Zn}$ & 8 & 73 & 5 & 11 & 6 & 15 & 163 \\
\hline $\begin{array}{c}\text { Coarse } \\
\mathrm{Al}\end{array}$ & 79 & 1971 & 64 & 116 & 36 & 69 & 440 \\
\hline $\begin{array}{c}\text { Coarse } \\
\mathrm{Si} \\
\end{array}$ & 245 & 1616 & 196 & 155 & 30 & 45 & 892 \\
\hline $\begin{array}{c}\text { Coarse } \\
\text { S }\end{array}$ & 38 & 8209 & 16 & 289 & 15 & 37 & 213 \\
\hline $\begin{array}{c}\text { Coarse } \\
\mathrm{Cl} \\
\end{array}$ & 159 & 31804 & 29 & 1187 & 19 & 34 & 428 \\
\hline $\begin{array}{c}\text { Coarse } \\
\mathrm{K}\end{array}$ & 48 & 7353 & 26 & 259 & 17 & 33 & 389 \\
\hline $\begin{array}{c}\text { Coarse } \\
\mathrm{Ca}\end{array}$ & 167 & 3600 & 147 & 159 & 22 & 27 & 892 \\
\hline $\begin{array}{c}\text { Coarse } \\
\text { Fe }\end{array}$ & 44 & 416 & 30 & 44 & 9 & 10 & 790 \\
\hline $\begin{array}{c}\text { Coarse } \\
\mathrm{Cu}\end{array}$ & 8 & 1177 & 3 & 45 & 4 & 14 & 113 \\
\hline
\end{tabular}


Table 11.3 $\quad \mathrm{PM}_{10}\left(\mu \mathrm{g} \mathrm{m}^{-3}\right)$, BC $\left(\mathrm{ng} \mathrm{m}^{-3}\right)$ and elemental $\left(\mathrm{ng} \mathrm{m}^{-3}\right)$ data from the Girl Guides site (1027 samples)

\begin{tabular}{|c|c|c|c|c|c|c|c|}
\hline & Average & Maximum & Median & $\begin{array}{l}\text { Standard } \\
\text { Deviation }\end{array}$ & $\begin{array}{c}\text { Average } \\
\text { Uncertainty }\end{array}$ & $\begin{array}{c}\text { Average } \\
\text { LOD }\end{array}$ & $\begin{array}{c}\text { Number }> \\
\text { LOD }\end{array}$ \\
\hline $\mathrm{PM}_{10}$ & 33 & 277 & 22 & 38 & & & \\
\hline Fine BC & 6553 & 19421 & 5476 & 3425 & 591 & 800 & 1027 \\
\hline Fine Al & 84 & 625 & 79 & 56 & 37 & 64 & 675 \\
\hline Fine Si & 205 & 733 & 173 & 117 & 28 & 42 & 1027 \\
\hline Fine S & 188 & 559 & 172 & 77 & 21 & 29 & 1027 \\
\hline Fine $\mathrm{K}$ & 254 & 1328 & 172 & 256 & 22 & 29 & 870 \\
\hline Fine Ca & 72 & 1443 & 62 & 74 & 18 & 26 & 907 \\
\hline Fine Fe & 36 & 178 & 26 & 30 & 8 & 9 & 918 \\
\hline Fine $\mathrm{Cu}$ & 3 & 159 & 0 & 8 & 3 & 13 & 110 \\
\hline Fine Zn & 14 & 131 & 10 & 16 & 8 & 16 & 360 \\
\hline $\begin{array}{c}\text { Coarse } \\
\mathrm{Al}\end{array}$ & 124 & 1309 & 104 & 107 & 41 & 68 & 773 \\
\hline $\begin{array}{c}\text { Coarse } \\
\text { Si }\end{array}$ & 334 & 2400 & 244 & 258 & 33 & 46 & 1027 \\
\hline $\begin{array}{c}\text { Coarse } \\
\mathrm{S}\end{array}$ & 50 & 669 & 35 & 67 & 18 & 35 & 535 \\
\hline $\begin{array}{c}\text { Coarse } \\
\mathrm{Cl}\end{array}$ & 380 & 12420 & 51 & 1044 & 26 & 33 & 674 \\
\hline $\begin{array}{c}\text { Coarse } \\
\mathrm{K}\end{array}$ & 67 & 642 & 49 & 70 & 19 & 32 & 677 \\
\hline $\begin{array}{c}\text { Coarse } \\
\mathrm{Ca} \\
\end{array}$ & 251 & 813 & 230 & 100 & 25 & 27 & 1027 \\
\hline $\begin{array}{c}\text { Coarse } \\
\text { Fe }\end{array}$ & 67 & 533 & 44 & 69 & 10 & 10 & 948 \\
\hline $\begin{array}{c}\text { Coarse } \\
\mathrm{Cu}\end{array}$ & 6 & 525 & 0 & 28 & 4 & 14 & 112 \\
\hline
\end{tabular}


Table 11.4 $\mathrm{PM}_{10}\left(\mu \mathrm{g} \mathrm{m}^{-3}\right)$, BC $\left(\mathrm{ng} \mathrm{m}^{-3}\right)$ and elemental $\left(\mathrm{ng} \mathrm{m}^{-3}\right)$ data from the Central Otago District Council site (940 samples)

\begin{tabular}{|c|c|c|c|c|c|c|c|}
\hline & Average & Maximum & Median & $\begin{array}{l}\text { Standard } \\
\text { Deviation }\end{array}$ & $\begin{array}{c}\text { Average } \\
\text { Uncertainty } \\
\end{array}$ & $\begin{array}{c}\text { Average } \\
\text { LOD } \\
\end{array}$ & $\begin{array}{c}\text { Number }> \\
\text { LOD }\end{array}$ \\
\hline $\mathrm{PM}_{10}$ & 17 & 136 & 9 & 22 & & & \\
\hline Fine BC & 4385 & 15563 & 3999 & 2065 & 540 & 800 & 940 \\
\hline Fine $\mathrm{Al}$ & 71 & 1536 & 64 & 79 & 33 & 61 & 520 \\
\hline Fine Si & 162 & 2266 & 130 & 136 & 25 & 38 & 937 \\
\hline Fine $S$ & 136 & 578 & 126 & 50 & 18 & 26 & 938 \\
\hline Fine K & 107 & 1917 & 60 & 149 & 16 & 25 & 730 \\
\hline Fine Ca & 53 & 848 & 46 & 45 & 15 & 22 & 794 \\
\hline Fine $\mathrm{Fe}$ & 30 & 328 & 24 & 27 & 7 & 9 & 820 \\
\hline Fine $\mathrm{Cu}$ & 3 & 328 & 1 & 12 & 3 & 12 & 149 \\
\hline Fine $\mathrm{Zn}$ & 8 & 168 & 5 & 12 & 5 & 15 & 163 \\
\hline $\begin{array}{c}\text { Coarse } \\
\mathrm{Al}\end{array}$ & 105 & 2387 & 86 & 136 & 39 & 69 & 606 \\
\hline $\begin{array}{c}\text { Coarse } \\
\text { Si }\end{array}$ & 247 & 4662 & 189 & 236 & 31 & 46 & 935 \\
\hline $\begin{array}{c}\text { Coarse } \\
\mathrm{S}\end{array}$ & 29 & 376 & 21 & 37 & 16 & 36 & 324 \\
\hline $\begin{array}{c}\text { Coarse } \\
\mathrm{Cl}\end{array}$ & 278 & 9212 & 37 & 817 & 22 & 35 & 510 \\
\hline $\begin{array}{c}\text { Coarse } \\
\mathrm{K}\end{array}$ & 37 & 546 & 28 & 42 & 16 & 35 & 443 \\
\hline $\begin{array}{c}\text { Coarse } \\
\mathrm{Ca} \\
\end{array}$ & 143 & 680 & 152 & 90 & 19 & 31 & 765 \\
\hline $\begin{array}{c}\text { Coarse } \\
\text { Fe }\end{array}$ & 42 & 1034 & 24 & 71 & 8 & 13 & 657 \\
\hline $\begin{array}{c}\text { Coarse } \\
\mathrm{Cu}\end{array}$ & 4 & 72 & 2 & 6 & 3 & 16 & 154 \\
\hline
\end{tabular}


Table 11.5 $\quad \mathrm{PM}_{10}\left(\mu \mathrm{g} \mathrm{m}^{-3}\right)$, BC $\left(\mathrm{ng} \mathrm{m}^{-3}\right)$ and elemental $\left(\mathrm{ng} \mathrm{m} \mathrm{m}^{-3}\right)$ data from the Aloft site (845 samples)

\begin{tabular}{|c|c|c|c|c|c|c|c|}
\hline & Average & Maximum & Median & $\begin{array}{l}\text { Standard } \\
\text { Deviation } \\
\end{array}$ & $\begin{array}{c}\text { Average } \\
\text { Uncertainty }\end{array}$ & $\begin{array}{c}\text { Average } \\
\text { LOD }\end{array}$ & $\begin{array}{c}\text { Number }> \\
\text { LOD } \\
\end{array}$ \\
\hline $\mathrm{PM}_{10}$ & 29 & 209 & 21 & 30 & & & \\
\hline Fine BC & 5545 & 18242 & 4840 & 2682 & 538 & 800 & 845 \\
\hline Fine Al & 80 & 1789 & 72 & 98 & 31 & 62 & 621 \\
\hline Fine Si & 156 & 1199 & 137 & 78 & 24 & 37 & 842 \\
\hline Fine S & 146 & 434 & 134 & 62 & 20 & 24 & 843 \\
\hline Fine K & 179 & 1330 & 107 & 203 & 15 & 27 & 733 \\
\hline Fine Ca & 24 & 471 & 19 & 28 & 15 & 23 & 797 \\
\hline Fine Fe & 31 & 294 & 25 & 25 & 6 & 8 & 821 \\
\hline Fine $\mathrm{Cu}$ & 3 & 252 & 0 & 11 & 3 & 13 & 123 \\
\hline Fine Zn & 12 & 388 & 7 & 26 & 4 & 15 & 184 \\
\hline $\begin{array}{c}\text { Coarse } \\
\mathrm{Al}\end{array}$ & 98 & 685 & 88 & 66 & 37 & 71 & 672 \\
\hline $\begin{array}{c}\text { Coarse } \\
\text { Si }\end{array}$ & 271 & 1202 & 225 & 147 & 32 & 44 & 845 \\
\hline $\begin{array}{c}\text { Coarse } \\
\text { S }\end{array}$ & 27 & 266 & 22 & 26 & 17 & 35 & 314 \\
\hline $\begin{array}{c}\text { Coarse } \\
\mathrm{Cl}\end{array}$ & 159 & 4097 & 49 & 346 & 23 & 33 & 534 \\
\hline $\begin{array}{c}\text { Coarse } \\
\text { K }\end{array}$ & 38 & 157 & 31 & 30 & 14 & 35 & 421 \\
\hline $\begin{array}{c}\text { Coarse } \\
\text { Ca }\end{array}$ & 158 & 1792 & 146 & 80 & 14 & 32 & 782 \\
\hline $\begin{array}{c}\text { Coarse } \\
\text { Fe }\end{array}$ & 45 & 429 & 32 & 42 & 7 & 12 & 699 \\
\hline $\begin{array}{c}\text { Coarse } \\
\mathrm{Cu}\end{array}$ & 4 & 72 & 0 & 6 & 3 & 15 & 121 \\
\hline
\end{tabular}

Reconstructed masses (RCMs) determined using the elemental data accounted for 26, 23, 27 and $21 \%$ of the $\mathrm{PM}_{10}$ mass at the ABC, GG, Aloft and CODC sites, respectively (Malm et al. 1994). Because numerous species, including organic carbon, were not quantified, the relatively low RCMs are not surprising. Sources that featured little contribution from unmeasured species (marine aerosol and crustal matter) had RCMs close to those identified from PMF. The application of PMF to hourly elemental data from each of the sites revealed four $\mathrm{PM}_{10}$ sources at each of the sites. The source profiles obtained for GG are presented in Figure 11.7, while the source profiles for ABC, CODC and Aloft are presented as Figures 11.8, 11.9 and 11.10, respectively. The error bars shown in Figures 11.7-11.10 indicate standard deviations 
determined from bootstrapping in the EPAPMF program. Limitations in the bootstrapping technique have previously been discussed (Ancelet et al. 2012b).

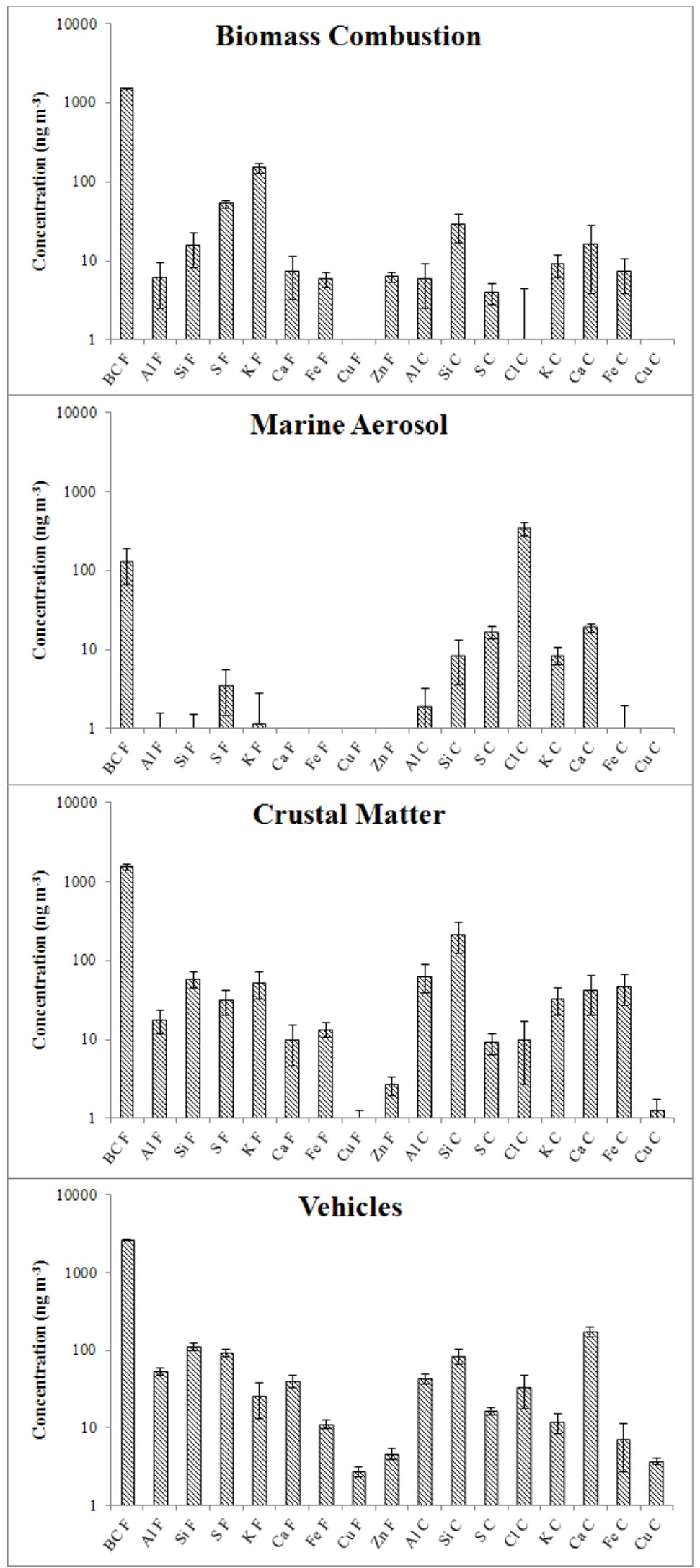

Figure 11.7 Source profiles obtained at the Alexandra Girl Guides site. 


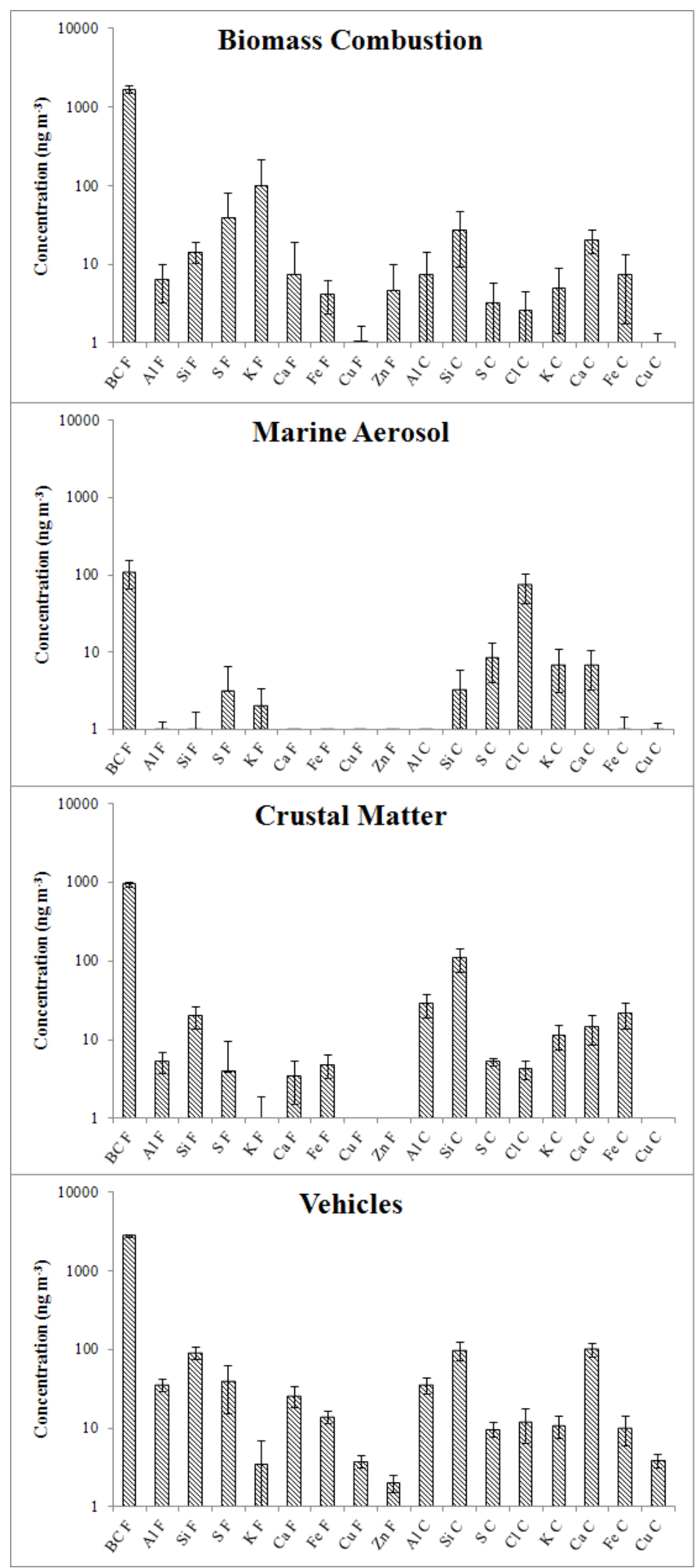

Figure 11.8 Source profiles obtained at the Alexandra Bowling Club site 


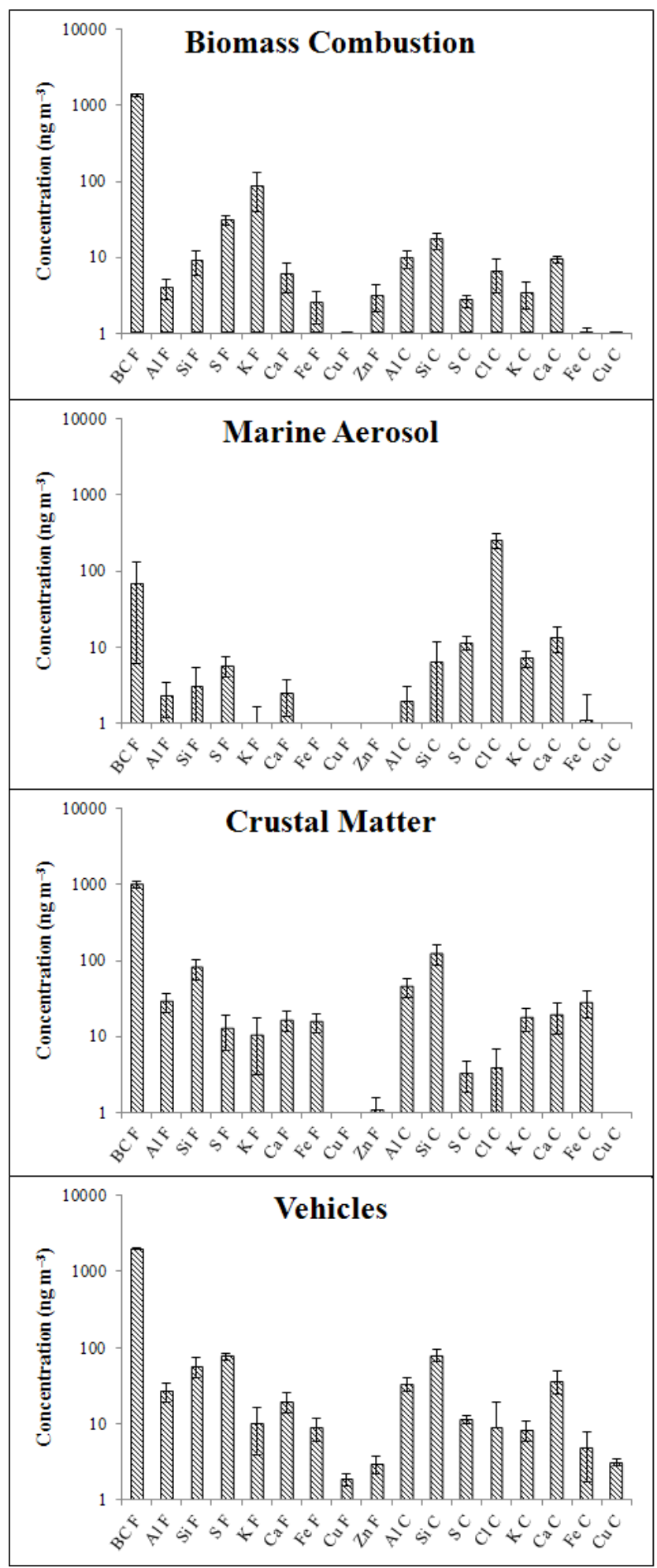

Figure 11.9 Source profiles obtained at the Central Otago District Council offices site 


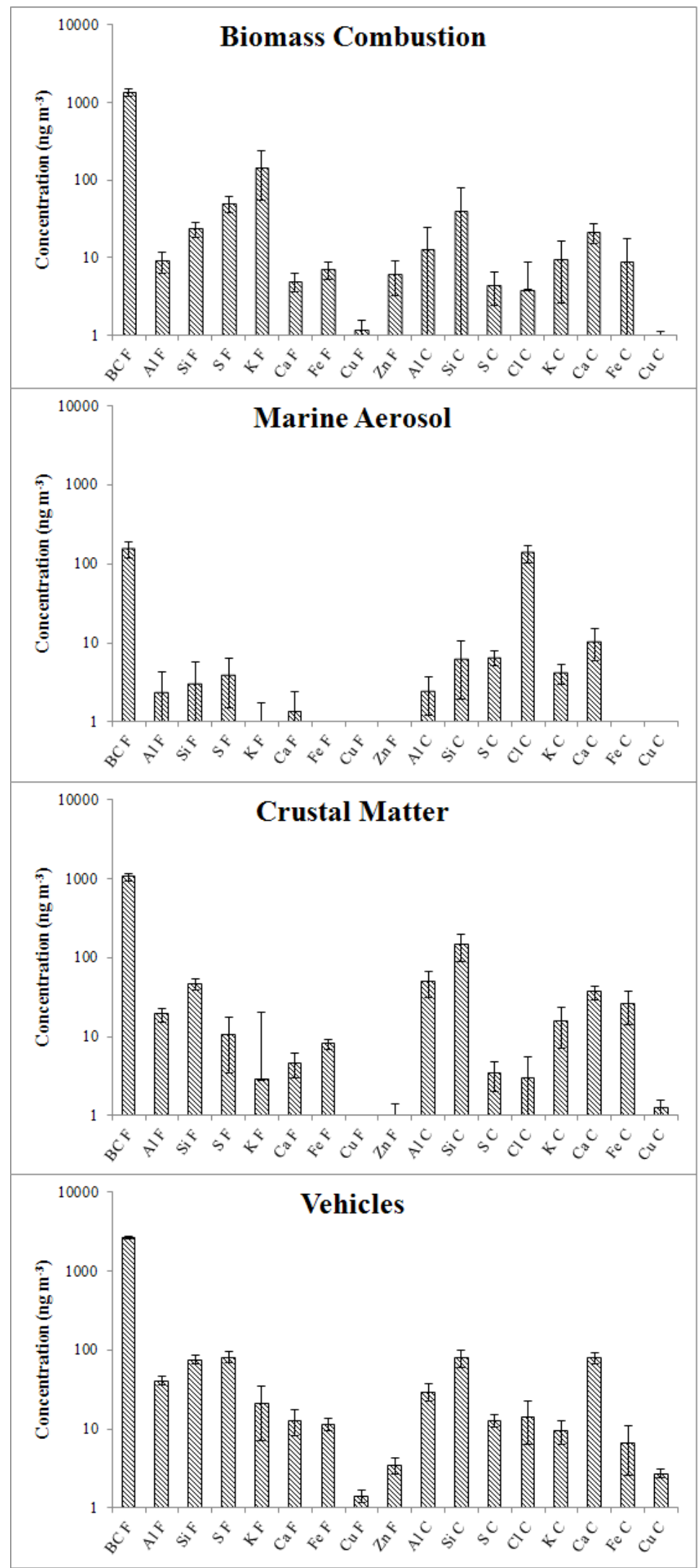

Figure 11.10 Source profiles obtained at the Aloft site 
The sources presented in Figures 11.7-11.10 were found to explain 96, 95, 98 and $99 \%$ of the $\mathrm{PM}_{10}$ mass measured by the E-BAMS at ABC, GG, Aloft and CODC, respectively, after regression using $\mathrm{PM}_{10}$ concentrations. Factor one contributed to 86, 90, 91 and $88 \%$ of the $\mathrm{PM}_{10}$ mass at ABC, GG, Aloft and CODC, respectively. It was identified as a biomass combustion source because of high $\mathrm{BC}$ and fine $\mathrm{K}$ loadings. Potassium is usually used alongside BC as a marker for biomass burning, and wood combustion in particular (Fine et al. 2002; Khalil and Rasmussen 2003).

The second factor was characterized as marine aerosol because of high $\mathrm{Cl}$ concentrations. The marine aerosol contribution to $\mathrm{PM}_{10}$ concentrations was 2, 1, 4 and $1 \%$ at $\mathrm{ABC}$, GG, Aloft and CODC, respectively. Marine aerosol is a common component in $\mathrm{PM}_{10}$ throughout New Zealand. The third factor was identified as soil, or crustal matter, and contributed to $1,1,1$ and $3 \%$ of the measured $\mathrm{PM}_{10}$ concentrations at ABC, GG, Aloft and CODC, respectively. The crustal matter source profile was characterized based on the presence of $\mathrm{Al}, \mathrm{Si}, \mathrm{K}, \mathrm{Ca}$ and $\mathrm{Fe}$. The higher contribution of crustal matter at CODC was likely the result of local roadworks that were taking place near the site.

Factor four accounted for $11,8,4$ and $8 \%$ of the $\mathrm{PM}_{10}$ mass at ABC, GG, Aloft and CODC, respectively. This factor was characterized as a vehicular source, which included vehicular exhaust and non-exhaust emissions (road dust). Road dust is generated by the turbulent passage of vehicles over local roads and the source profiles feature crustal elements ( $\mathrm{Al}$ and $\mathrm{Si}$ ) enriched with $\mathrm{BC}, \mathrm{Ca}$ and $\mathrm{Fe}$. The vehicle source profiles reported here are consistent with those reported previously (Garg et al. 2000; Schauer et al. 2006). Black carbon in the vehicle profiles can be associated with exhaust emissions, deposited tailpipe emissions and the abrasion of tar-sealed surfaces. Iron and copper are typically present in brake wear dust (Thorpe and Harrison 2008). The higher average contribution from vehicles at $A B C$ is not surprising, since the site was located close to the main road passing through Alexandra.

The average hourly source contributions at each site were calculated to assess variations in source contributions on an hourly time-scale. Figure 11.11 presents the average hourly source contributions at GG and Figures 11.12, 11.13 and 11.14 present the average hourly source contributions at ABC, CODC and Aloft, respectively. A number of notable features are apparent from Figures 11.11-11.14. First, biomass combustion is a significant PM source every hour during the winter and was 
responsible for both peaks (evening and morning) observed in the $\mathrm{PM}_{10}$ diurnal cycle. This phenomenon was also observed in Masterton, New Zealand (Ancelet et al. 2012b), and suggests that Alexandra residents are relighting their fires when they rise in the morning. It was suggested by Trompetter et al. (2010) that the morning peak could also result from built-up $\mathrm{PM}_{10}$ above the inversion layer being re-entrained to ground level by atmospheric mixing upon the break-up of the inversion, which has been reported previously (Aryal et al. 2009). Based on measurements from the Aloft site and ground observations (no decreased visibility during the early morning), this mechanism was ruled out. We therefore suggest that katabatic flows result in dispersion and a consequent decrease in $\mathrm{PM}_{10}$ concentrations during the early morning when there are fewer new particle emissions from biomass combustion. The morning peak then arises from fires that are lit or restoked in the morning.

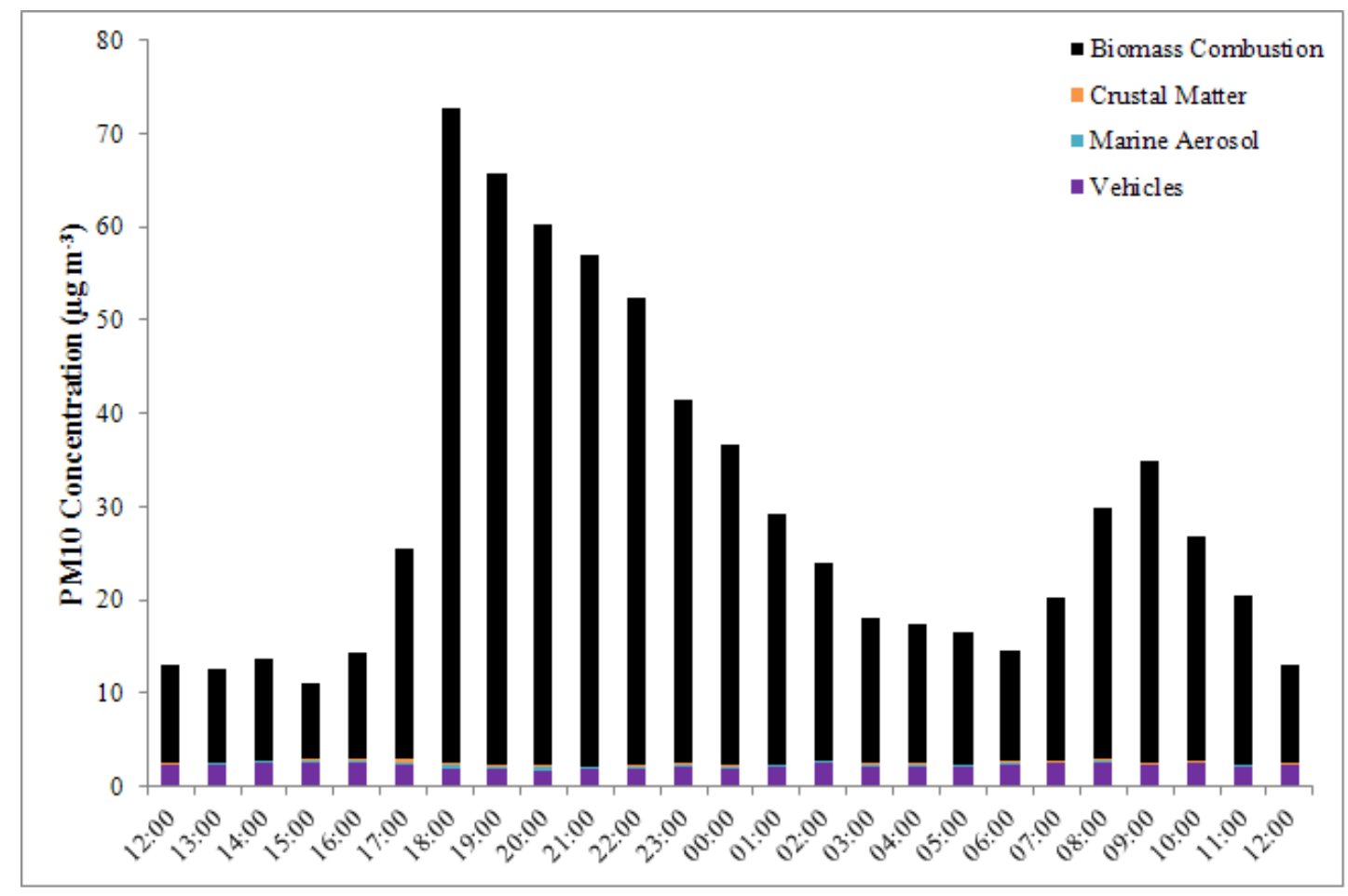

Figure 11.11 Average hourly source contributions at the Alexandra Girl Guides site. 


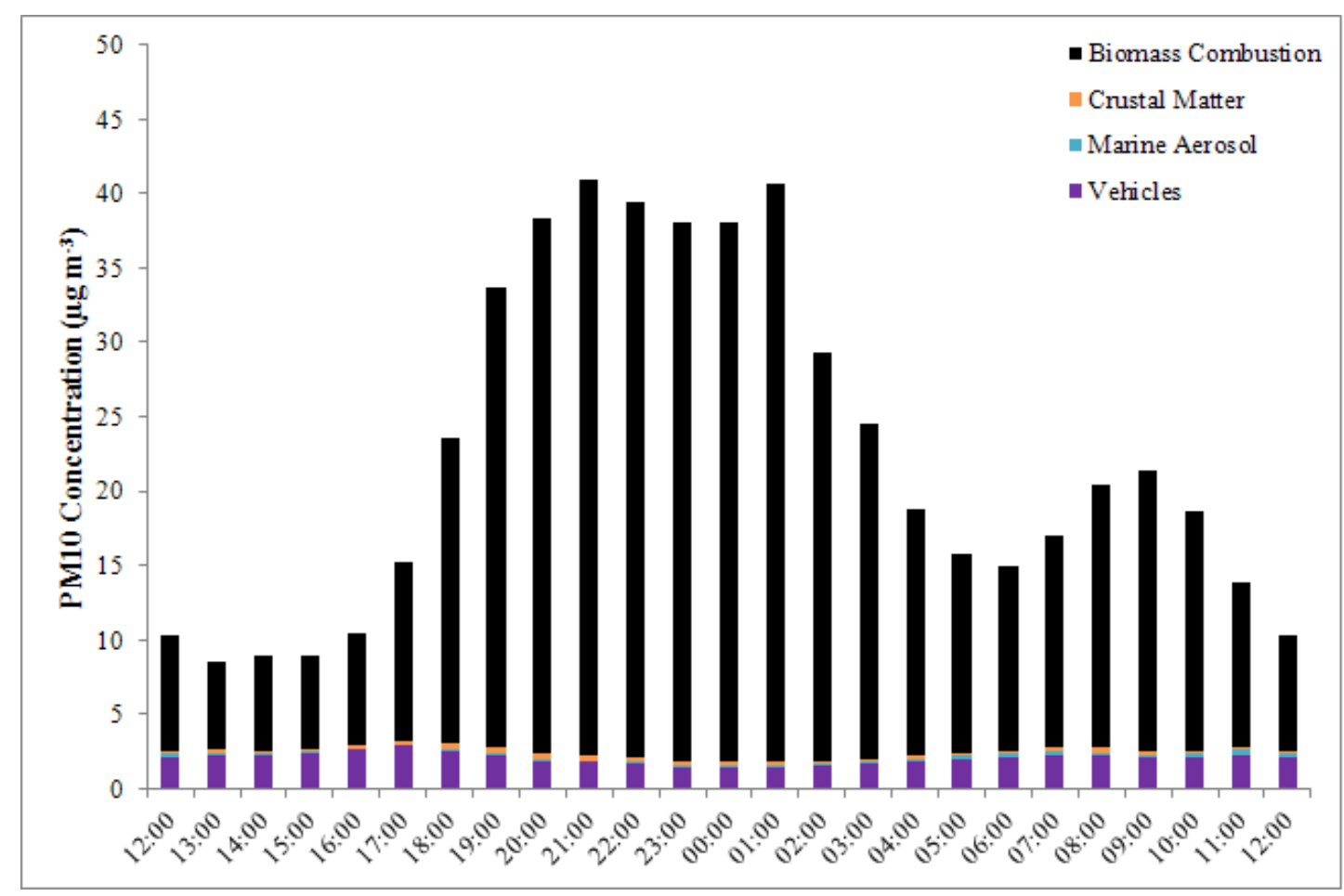

Figure 11.12 Average hourly source contributions at the Alexandra Bowling Club site.

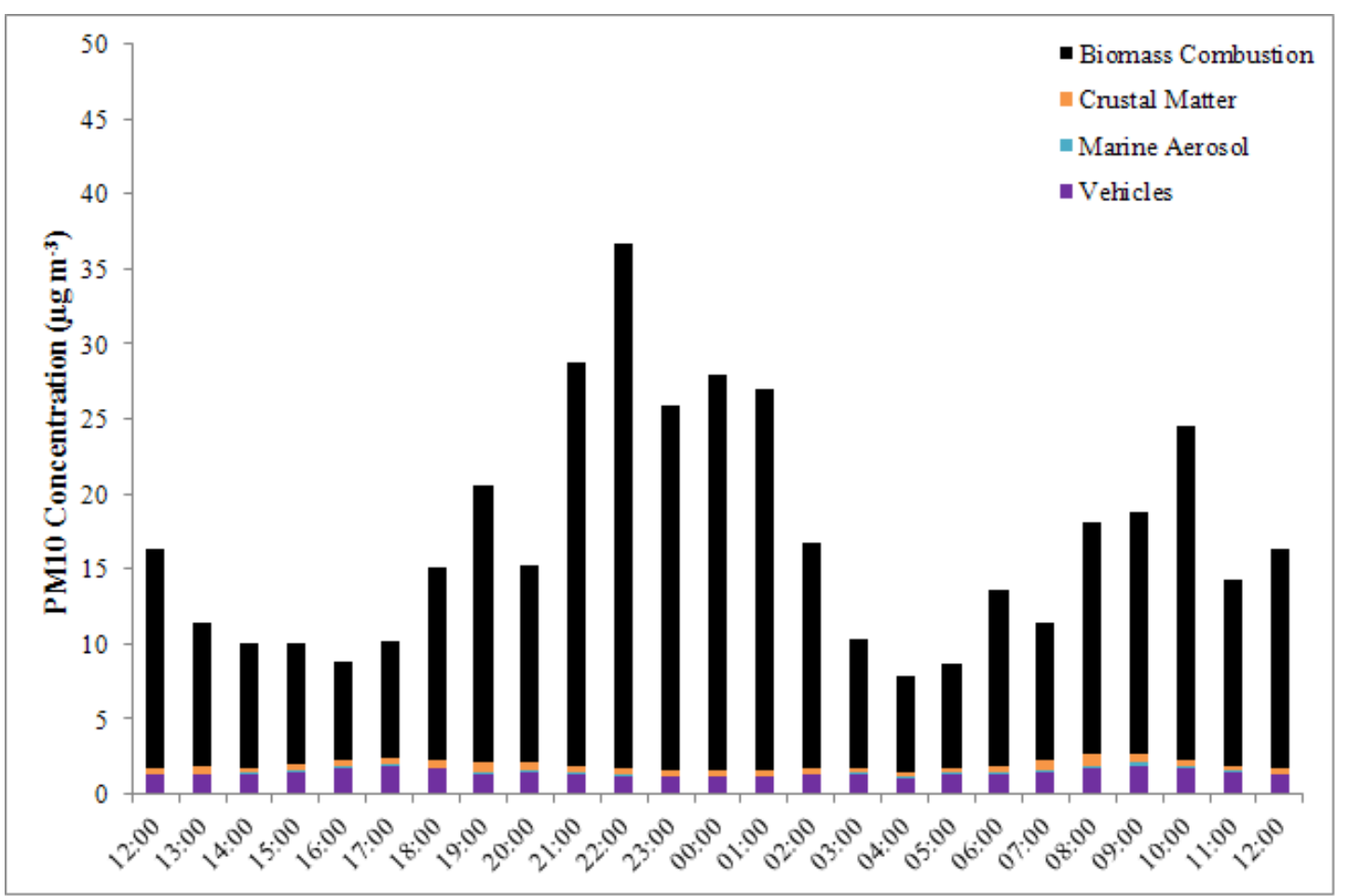

Figure 11.13 Average hourly source contributions at the Central Otago District Council site. 


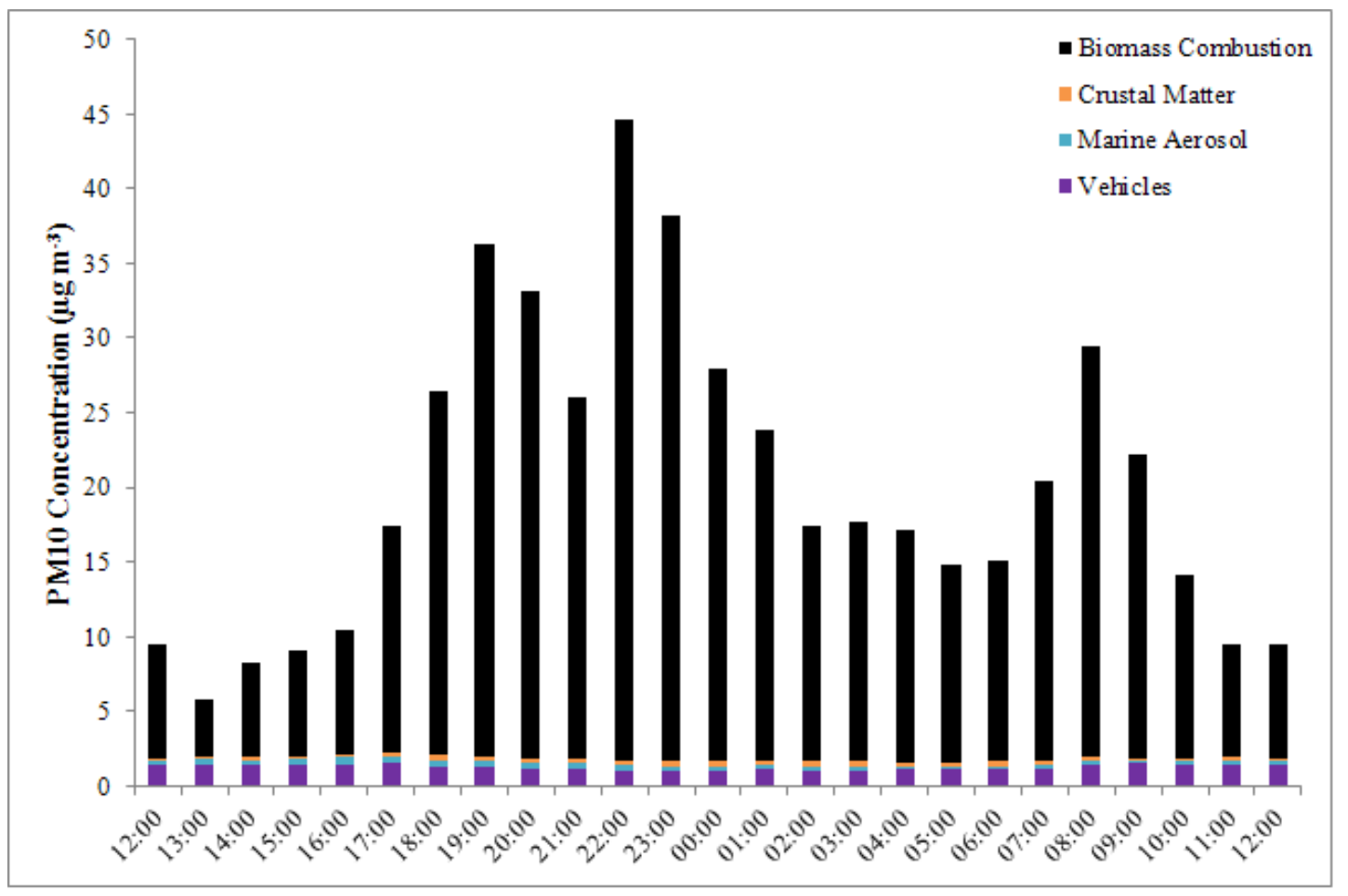

Figure 11.14 Average hourly source contributions at the Aloft site.

\subsubsection{Source Transport}

Polar plots using the hourly biomass combustion contributions were prepared to further investigate the transport mechanism and potential biomass combustion source locations (Carslaw and Ropkins 2011; R Development Core Team 2011). Using polar plots, the biomass combustion contributions can be plotted as a function of both wind speed and direction. To gain insight into the observed diurnal variations, the source contribution data were divided into night ( $6 \mathrm{pm}-8 \mathrm{am})$ and day (9 am-5 $\mathrm{pm}$ ). It is important to note is that the biomass combustion contributions (in $\mu \mathrm{g} \mathrm{m}^{-3}$ ) should not be taken as the actual concentration. The values actually indicate the average concentration for each wind speed/direction bin. Figure 11.15 (a) and (b) presents biomass combustion polar plots obtained using data from the night and day at the GG site. 

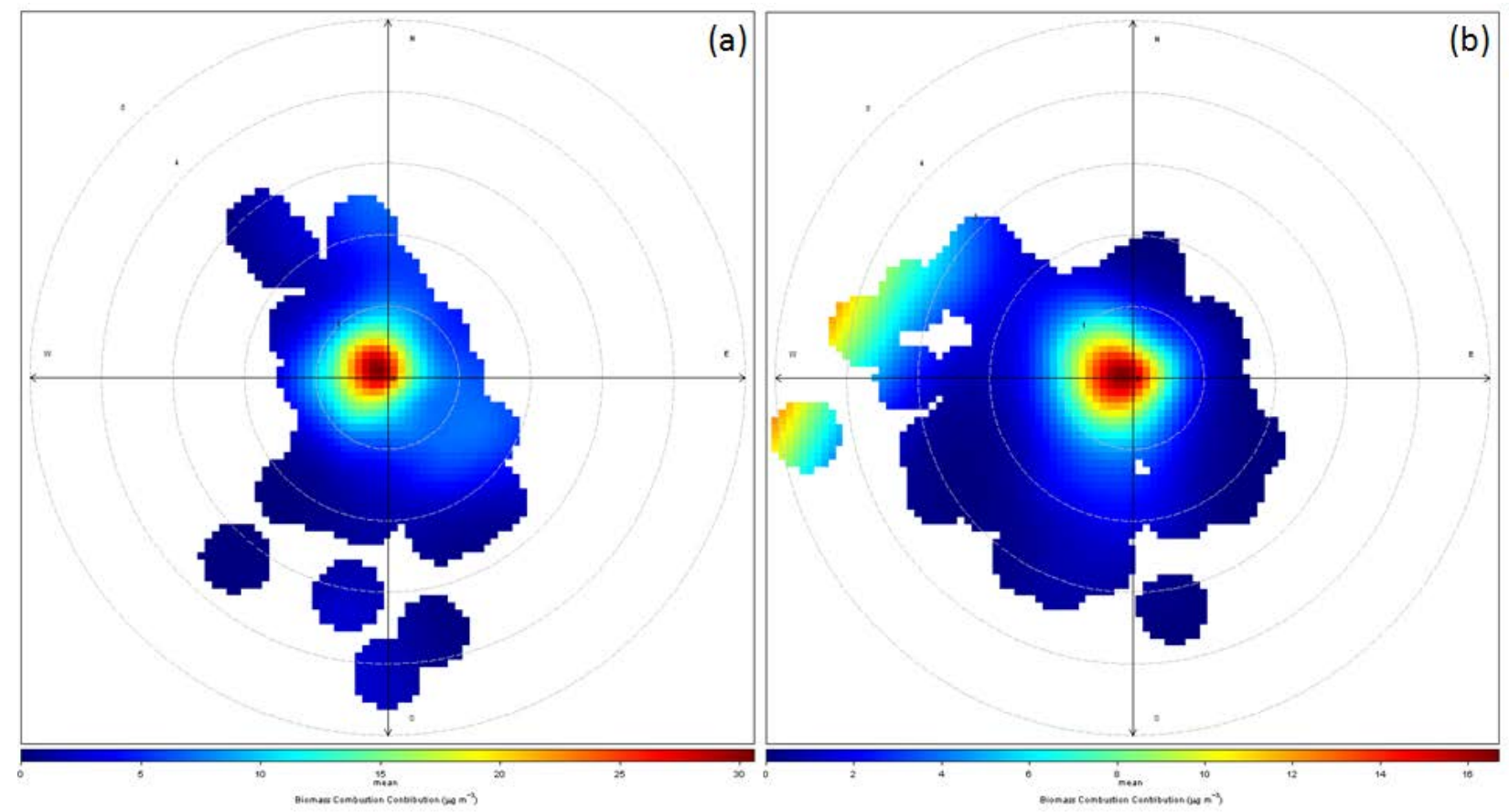

Figure 11.15 Polar plots of biomass combustion contributions during the night (a) and day (b) at the GG site. The radial dimensions indicate the wind speed in $1 \mathrm{~ms}^{-1}$ increments and the color contours indicate the average contribution to each wind direction/speed bin.

Figure 11.15 (a) shows that during the night the highest biomass combustion contributions were the result of transport from the northwest when wind speeds were greater than $1 \mathrm{~m} \mathrm{~s}^{-1}$, and, when wind speeds were very low $\left(<1 \mathrm{~m} \mathrm{~s}^{-1}\right)$, little directionality was apparent, supporting the suggestion from Chapter 11.4.1 that GG was located at the convergence point of a number of different katabatic flows. Figure 11.15 (b) also indicates that peak biomass combustion contributions showed little directionality. Under high wind speeds from the west, moderate biomass combustion contributions were also evident during the day. An older residential area with a high concentration of wood burners was west of the GG site, suggesting that at least some homes continued to burn wood throughout the day.

Contributions from vehicular sources at each of the sites increased during peak traffic hours. Vehicle contributions were also apparent during hours when traffic flows would be expected to be minimal. Although Alexandra is a small community, a highway runs through the town and it is likely that, heavy-duty diesel vehicles in particular, provide a consistent $\mathrm{PM}_{10}$ source throughout the day. Further support for this conclusion was provided by polar plots of vehicle contributions during the day and night. Figure 11.16 (a) and (b) presents polar plots of vehicle contributions during 
the night and day, respectively. During the day, peak vehicle contributions at GG were transported from the direction of the town center, where local traffic movements during the day would be highest. Overall, vehicle contributions during the day are indicative of local traffic movements along main roads within the town. During the night (Figure 11.16 (a)), vehicle contributions were largely from the east and northwest. This result suggests that traffic along the highway passing through Alexandra was largely responsible for vehicle contributions during the evening.

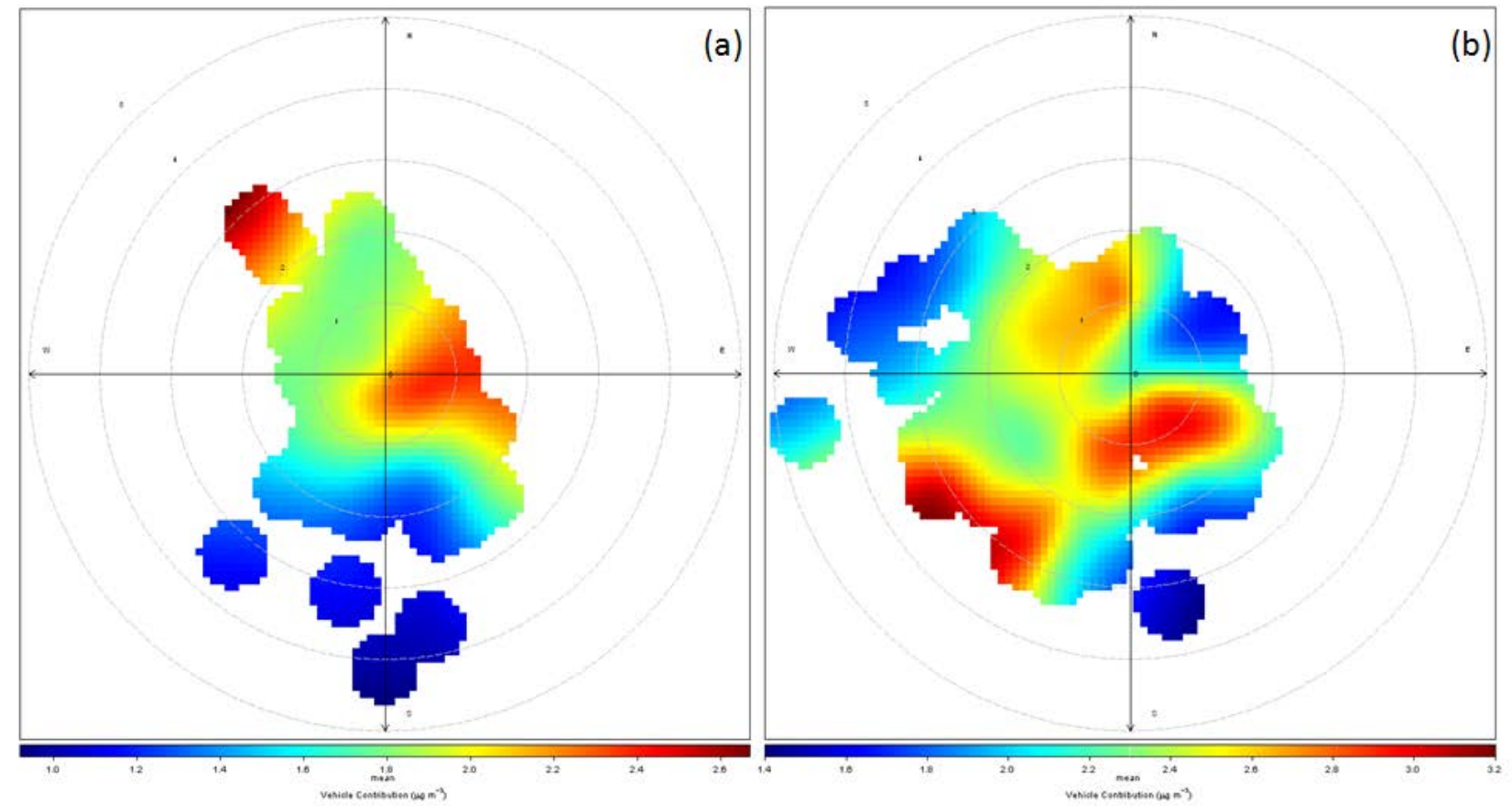

Figure 11.16 Polar plots of vehicle contributions during the night (a) and day (b) at the GG site. The radial dimensions indicate the wind speed in $1 \mathrm{~ms}^{-1}$ increments and the color contours indicate the average contribution to each wind direction/speed bin.

Marine aerosol contributions were more pronounced when hourly wind speeds were highest and originated from the south. It is well-known that marine aerosol contributions increase under increased wind speeds (Fitzgerald 1991). Day and night polar plots of marine aerosol and crustal matter contributions are presented in Figures 11.17 and 11.18, respectively. 

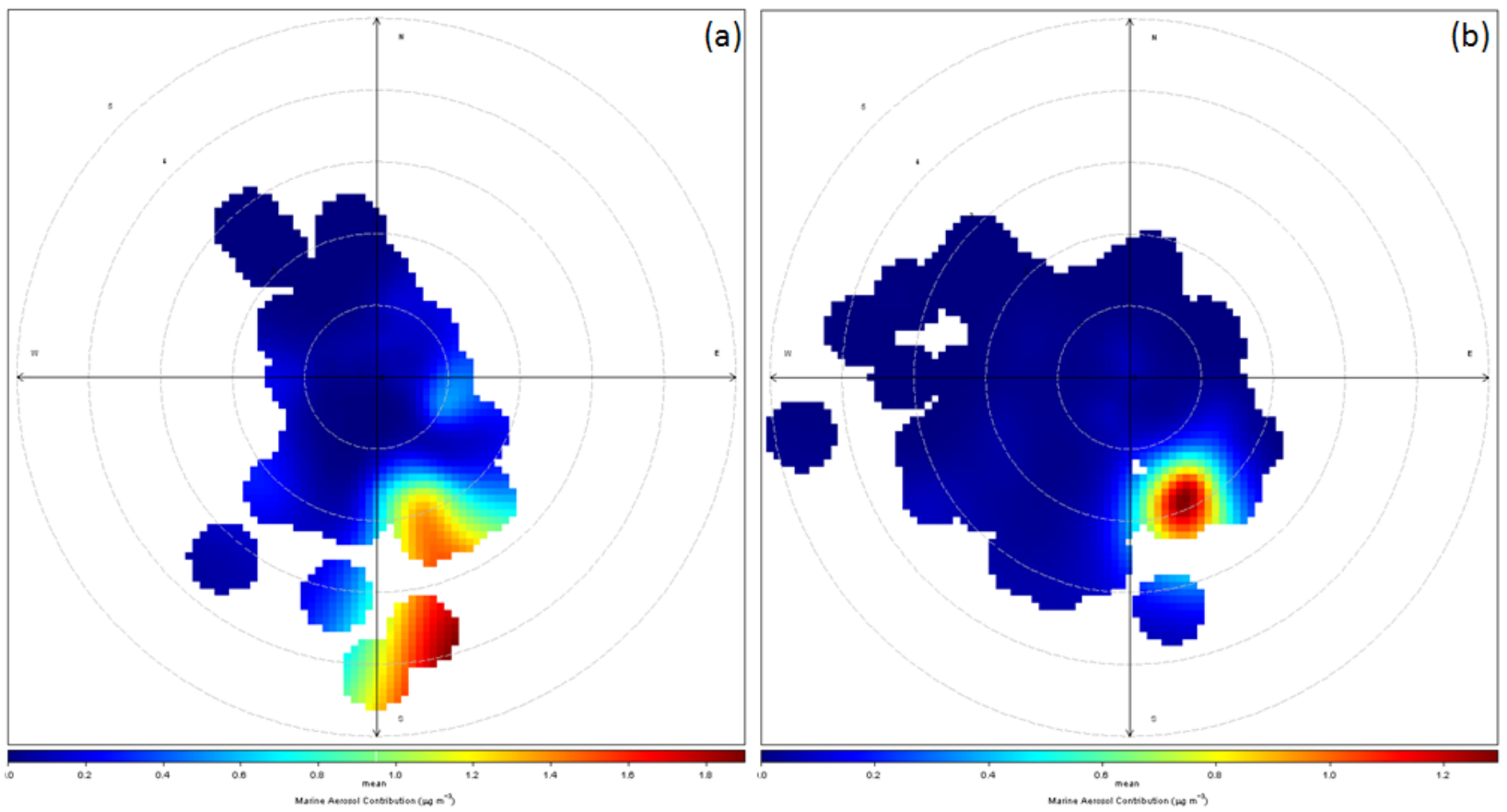

Figure 11.17 Polar plots of marine aerosol contributions during the night (a) and day (b) at the GG site. The radial dimensions indicate the wind speed in $1 \mathrm{~ms}^{-1}$ increments and the color contours indicate the average contribution to each wind direction/speed bin.
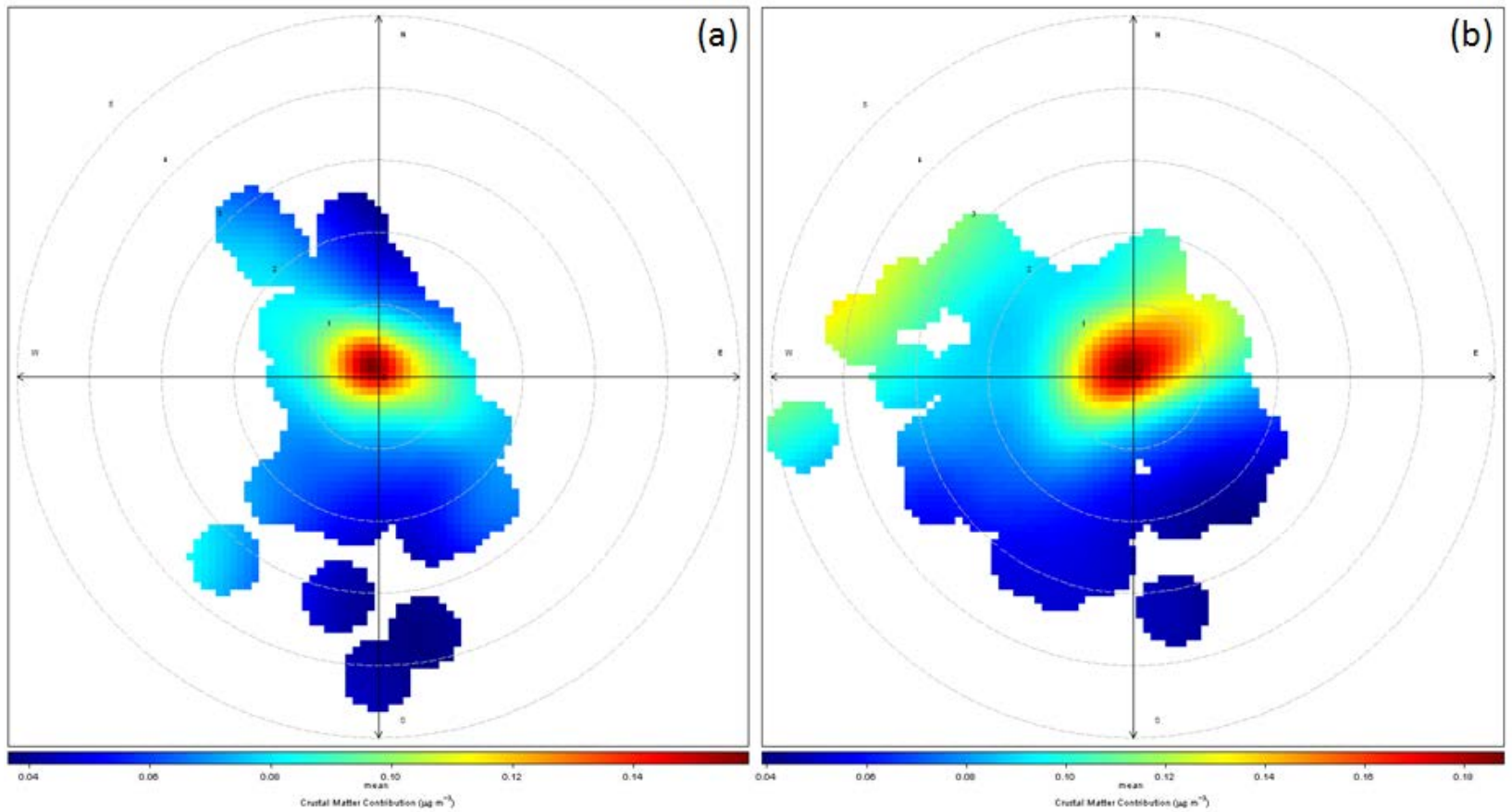

Figure 11.18 Polar plots of crustal matter contributions during the night (a) and day (b) at the GG site. The radial dimensions indicate the wind speed in $1 \mathrm{~ms}^{-1}$ increments and the color contours indicate the average contribution to each wind direction/speed bin.

Typically crustal matter contributions also increase under increased wind speeds, but Figure 11.18 indicates that the highest crustal matter contributions 
occured during low wind speeds. This suggests that the suspended crustal matter probably originated locally, likely as a result of re-suspension by vehicles.

\subsection{Acknowledgments}

The support of Otago Regional Council (Deborah Mills) was greatly appreciated. We thank Bruce Crothers and Ed Hutchinson for their support in maintaining the sampling equipment and Chris Purcell for setting up and maintaining the $3 \mathrm{MeV}$ accelerator used for IBA. Stuart Grange is also thanked for his support during sampling. This work was funded by the Ministry of Science and Innovation under contract C05X0903. 


\section{Concluding Remarks}

The overarching goal of this thesis was to identify the PM sources responsible for the observed wintertime diurnal $\mathrm{PM}_{10}$ cycles in New Zealand's urban areas. $\mathrm{PM}_{10}$ in three different urban areas (Masterton, Alexandra and Nelson) that featured varying topographies and population densities was studied at an hourly time-scale. The sources contributing to the observed diurnal cycles at a number of locations within each urban area were successfully identified, making this work the first of its kind. Using multiple site locations within each urban area also allowed for investigations into how $\mathrm{PM}_{10}$ concentrations varied horizontally and vertically and insight into the transport mechanisms responsible for those variations.

\subsection{Key Findings}

- It is possible to combine Streaker samplers and E-BAMs for the identification of PM sources and their contributions on an hourly timescale.

- Overall, residential wood combustion for home heating was identified to be a dominant contributor to $\mathrm{PM}_{10}$ during every hour and was the principal source responsible for the observed evening and morning peaks in $\mathrm{PM}_{10}$ concentrations. This result is important because it has two major implications. First, it suggests that residents of these urban areas are re-lighting their fires in the morning. Second, since these peak $\mathrm{PM}_{10}$ concentrations are the main contributors to exceedances of the NES, dramatic reductions in emissions from solid fuel combustion should significantly improve air quality in the urban areas studied.

- A number of PM sources were common among Masterton, Alexandra and Nelson. These included: biomass burning, vehicles, crustal matter and marine aerosol. Shipping sulfate was only identified in Nelson.

- In each urban area katabatic flows along downslope contours were found to be responsible for elevated $\mathrm{PM}_{10}$ concentrations measured downwind. In Masterton, the downwind site located at Chanel College experienced higher 
$\mathrm{PM}_{10}$ concentrations than the Wairarapa College site which is used for compliance monitoring by the Greater Wellington Regional Council, suggesting that the council is not monitoring in a location that experiences maximum $\mathrm{PM}_{10}$ concentrations. $\mathrm{PM}$ transport in Alexandra was unique among the urban areas studied. Numerous katabatic flows converging from different directions were found to be responsible for peak $\mathrm{PM}_{10}$ concentrations occurring at the central (Girl Guides) site.

- In each urban area it was also clear that during the early morning the boundary layer had been effectively cleared. As a result, the morning peak in PM concentrations was the result of new emissions.

- $\mathrm{PM}_{10}$ concentrations measured aloft (at $26 \mathrm{~m}$ ) in Alexandra and Nelson were lower than those measured at ground level. Combined with higher wind speeds and temperatures, it was apparent that these elevated sites were generally above low-lying inversions that resulted in high $\mathrm{PM}_{10}$ concentrations at ground level.

- Marine aerosol contributions among the locations increased with decreasing distance from the ocean (Nelson $>$ Masterton > Alexandra).

- Vehicle contributions among the locations increased with increasing population (Nelson > Masterton > Alexandra).

\subsection{Future Directions}

This work has highlighted that it is possible to identify the sources of PM at an hourly resolution. It is envisioned that more studies like those reported in this thesis in other urban areas will provide invaluable information for air quality management. In particular, hourly monitoring over the course of a year would allow for the identification of seasonal differences in PM concentrations, sources and source contributions. Combining the Streaker sampler and E-BAM information with a realtime instrument, like an aerosol mass spectrometer, would provide important information about organic species and secondary organic aerosols that are not 
identified using IBA techniques. This information would not only allow for a better mass closure, but would significantly improve the ability of PMF to identify minor sources of PM.

Hourly PM sampling can also be targeted to specific source emissions, for example, vehicular emissions in a tunnel to better understand non-exhaust particle emissions. 


\section{References}

Ackerman, A. S., Toon, O. B., Stevens, D. E., Heymsfield, A. J., Ramanathan, V. and Welton, E. J., 2000, Reduction of tropical cloudiness by soot. Science, 288, 1042-1047.

Albrecht, B. A., 1989, Aerosols, cloud microphysics, and fractional cloudiness. Science, 245, 1227-1230.

Allen, J. O., Mayo, P. R., Hughes, L. S., Salmon, L. G. and Cass, G. R., 2001, Emissions of size-segregated aerosols from on-road vehicles in the Caldecott tunnel. Environmental Science and Technology, 35(21), 4189-97.

Ancelet, T., Davy, P. K., Trompetter, W. J., Markwitz, A. and Weatherburn, D. C., 2010, A comparison of particulate and particle-phase PAH emissions from a modern wood burner with those of an old wood burner. Air Quality and Climate Change, 44, 21-24.

Ancelet, T., Davy, P., K., Trompetter, W. J., Markwitz, A. and Weatherburn, D. C., 2011a, Characterisation of particulate matter emissions from a modern wood burner under varying burner conditions. Air Quality and Climate Change, 45, 21-27.

Ancelet, T., Davy, P. K., Trompetter, W. J., Markwitz, A. and Weatherburn, D. C., 2011a, Carbonaceous aerosols in an urban tunnel. Atmospheric Environment, 45(26), 4463-4469.

Ancelet, T., Davy, P. K., Mitchell, T., Trompetter, W. J., Markwitz, A. and Weatherburn, D. C., 2012b, Identification of particulate matter sources on an hourly time-scale in a wood burning community. Environmental Science and Technology, 46(9), 4767-4774.

Ancelet, T., Davy, P., K., Trompetter, W. J., Markwitz, A. and Weatherburn, D. C., 2012b, Sources of particulate matter on an hourly time-scale during the winter in a small city. Environmental Science and Technology, submitted.

Andreae, M. O. and Gelencśer, A., 2006, Black carbon or brown carbon? The nature of light-absorbing carbonaceous aerosols. Atmospheric Chemistry and Physics, 6(10), 3131-3148. 
Annegarn, H. J., Cahill, T. A., Sellschop, J. F. P. and Zucchiatti, A., 1988, Time sequence particulate sampling and nuclear analysis. Physics Scripta, 37, 282290.

Annegarn, H. J., Marcazzan, G. M. B., Cereda, E., Marchionni, M. and Zucchiatti, A., 1992, Source profiles by unique ratios (spur) analysis: Determination of source profiles from receptor-site streaker samples. Atmospheric Environment, 26A, 333-343.

Annegarn, H. J., Flanz, M., Kenntner, T., Kneen, M. A., Helas, G. and Piketh, S. J., 1996, Airborne streaker sampling for PIXE analysis. Nuclear Instruments and Methods in Physics Research, Section B: Beam Interactions with Materials and Atoms, 109-110, 548-550.

Appel, B. R., Tokiwa, Y., Hsu, J., Kothny, E. L. and Hahn, E., 1985, Visibility as related to atmospheric aerosol constituents. Atmospheric Environment, 19(9), $1525-1534$.

Aryal, R. K., Lee, B.-K., Karki, R., Gurung, A., Baral, B. and Byeon, S.-H., 2009, Dynamics of PM2.5 concentrations in Kathmandu Valley, Nepal. Journal of Hazardous Materials, 168(2-3), 732-738.

Ault, A. P., Moore, M. J., Furutani, H. and Prather, K. A., 2009, Impact of emissions from the Los Angeles Port region on San Diego air quality during regional transport events. Environmental Science and Technology, 43(10), 3500-3506.

Bae, M-S., Schauer, J. J., Turner, J. R. and Hopke, P. K., 2009, Seasonal variations of elemental carbon in urban aerosols as measured by two common thermaloptical carbon methods. Science of the Total Environment, 407, 5176-5183.

Bachmann, J. D., 2007, Will the circle be unbroken: A history of the US national ambient air quality standards - 2007 Critical Review. Journal of the Air and Waste Management Association, 57(6), 652-697.

Baldwin, D. P., Zamzow, D. S. and D'Silva, A. P., 1994, Aerosol mass measurement and solution standard additions for quantitation in laser ablation-inductively coupled plasma atomic emission spectrometry. Analytical Chemistry, 66, 1911-1917.

Bates, M., Bruno, P., Caputi, M., Caselli, M., de Gennaro, G. and Tutino, M., 2008, Analysis of polycyclic aromatic hydrocarbons (PAHs) in airborne particles by direct sample introduction thermal desorption GC/MS. Atmospheric Environment, 42(24), 6144-6151. 
Berube, K., Jones, T., Williamson, B. J., Winters, C., Morgan, A. J. and Richards, R. J., 1999, Physiochemical characterisation of diesel exhaust particles: Factors for assessing biological activity. Atmospheric Environment, 33, 1599-1614.

Birch, M. E. and Cary, R. A., 1996, Elemental carbon-based method for monitoring occupational exposures to particulate diesel exhaust. Aerosol Science and Technology, 25, 221-241.

Bond, T. C. and Bergstrom, R. W., 2006, Light absorption by carbonaceous particles: An investigative review. Aerosol Science and Technology, 40(1), 27-67.

Breed, C. A., Arocena, J. M. and Sutherland, D., 2002, Possible sources of PM10 in Prince George (Canada) as revealed by morphology and in situ chemical composition of particulate. Atmospheric Environment, 36(10), 1721-1731.

Broadbent, A., Cullen, N. J. and Zawar-Reza, P., 2010, Wintertime numerical modelling of PM10 air pollution in Milton, Otago, New Zealand: Boundary layer structure, effects of data assimilation, and reaching national environmental standards. Air Quality and Climate Change, 44, 22-28.

Brown, L. E., Trought, K. R., Bailey, C. I. and Clemons, J. H., 2005, 2,3,7,8-TCDD equivalence and mutagenic activity associated with PM10 from three urban locations in New Zealand. Science of the Total Environment, 349, 161-174.

Cadle, S. H., Mulawa, P. A., Hunsanger, E. C., Nelson, K., Ragazzi, R. A., Barrett, R., Gallagher, G. L., Lawson, D. R., Knapp, K. T. and Snow, R., 1999, Composition of Light-Duty Motor Vehicle Exhaust Particulate Matter in the Denver, Colorado Area. Environmental Science and Technology, 33(14), 2328-2339.

Cao, J. J., Lee, S. C., Ho, K. F., Zhang, X. Y., Zou, S. C., Fung, K., Chow, J. C. and Watson, J. G., 2003, Characteristics of carbonaceous aerosol in Pearl River Delta Region, China during 2001 winter period. Atmospheric Environment, 37(11), 1451-1460.

Carslaw, D. C. and Ropkins, K. (2011). Open-source tools for analysing air pollution data, Environmental Research Group, King's College London, 11th July 2011.

Cass, G. R., 1998, Organic molecular tracers for particulate air pollution sources. Trends in Analytical Chemistry, 17(6), 356-366.

Cavanagh, J.-A. E., Brown, L., Trought, K., Kingham, S. and Epton, M. J., 2007, Elevated concentrations of 1-hydroxypyrene in schoolchildren during winter 
in Christchurch, New Zealand. Science of the Total Environment, 374(1), 5159.

Cavanagh, J.-A. E., Trought, K., Brown, L. and Duggan, S., 2009, Exploratory investigation of the chemical characteristics and relative toxicity of ambient air particulates from two New Zealand cities. Science of the Total Environment, 407(18), 5007-5018.

Celis, J., Morales, R., Zaror, C., Inzunza, J., Flocchini, R. and Carvacho, O., 2003, Chemical characterization of the inhalable particulate matter in the city of Chillan, Chile. Journal of the Chilean Chemical Society, 48(2), 49-55.

Chellam, S., Kulkarni, P. and Fraser, M. P., 2005, Emissions of organic compounds and trace metals in fine particulate matter from motor vehicles: a tunnel study in Houston, Texas. Journal of the Air and Waste Management Association, 55, 60-72.

Cheng, Y., Zheng, M., He, K-B., Chen, Y., Yan, B., Russell, A. G., Shi, W., Jiao, Z., Sheng, G., Fu, J. and Edgerton, E. S., 2011, Comparison of two thermaloptical methods for the determination of organic carbon and elemental carbon: results from the Southeastern United States. Atmospheric Environment, 45, 1913-1918.

Chow, J. C., Watson, J. G., Pritchett, L. C., Pierson, W. R., Frazier, C. A. and Purcell, R. G., 1993, The DRI thermal/optical reflectance carbon analysis system: description, evaluation and applications in U.S. air quality studies. Atmospheric Environment, 27A(8), 1185-1201.

Chow, J. C. and Watson, J. G., 1999, Ion chromatography in elemental analysis of airborne particles. Advances in Environmental, Industrial and Process Control Technologies, 1: Elemental Analysis of Airborne Particles, 97-137.

Chow, J. C., Watson, J. G., Crow, D., Lowenthal, D. H. and Merrifield, T., 2001, Comparison of IMPROVE and NIOSH carbon measurements. Aerosol Science and Technology, 34(1), 23-34.

Chow, J. C., Watson, J. G., Chen, L-W. A., Chang, M. C. O., Robinson, N. F., Trimble, D. L. and Kohl, S. D., 2007a, The IMPROVE_A temperature protocol for thermal/optical carbon analysis: Maintaining consistency with a long-term database. Journal of the Air and Waste Management Association, 57(9), 1014-1023. 
Chow, J. C., Watson, J. G., Ho, S. S. H., Bohannan, T. L., Hays, M. D. and Fung, K. K., 2007b, The application of thermal methods for determining chemical composition of carbonaceous aerosols: A review. Journal of Environmental Science and Health - Part A, 42(11), 1521-1541.

Cohen, D. (1999). Accelerator based ion beam techniques for trace element aerosol analysis. Elemental Analysis of Airborne Particles. Landsberger, S. and Creatchman, M. eds. Amsterdam, Gordon and Breach Science Publishers. 139-196.

Colbeck, I. and Lazaridis, M., 2010, Aerosols and environmental pollution. Naturwissenschaften, 97(2), 117-131.

Conway, J., Spronken-Smith, R. A. and Zawar-Reza, P., 2007, The clean air down there? Spatial patterns of particulate matter pollution in Invercargill, New Zealand. Air Quality and Climate Change, 41, 19-25.

Cretney, J. R., Lee, H. K., Wright, G. J., Swallow, W. H. and Taylor, M. C., 1985, Analysis of polycyclic aromatic hydrocarbons in air particulate matter from a lightly industrialized urban area. Environmental Science and Technology, 19(5), 397-404.

D'Alessandro, A., Lucarelli, F., Manda, P. A., Marcazzan, G., Nava, S., Prati, P., Valli, G., Vecchi, R. and Zucchiatti, A., 2003, Hourly elemental composition and sources identification of fine and coarse PM10 particulate matter in four Italian towns. Journal of Aerosol Science, 34(2), 243-259.

D'Alessandro, A., Nava, S., Van Ham, R., Adriaens, A., Lucarelli, F., Marcazzan, G., Prati, P., Valli, G., Vecchi, R. and Zucchiatti, A., 2004, PIXE and ToF-SIMS analysis of streaker samplers filters. Nuclear Instruments and Methods in Physics Research, Section B: Beam Interactions with Materials and Atoms, 222(1-2), 261-269.

Davy, P. K., Markwitz, A., Trompetter, W. J. and Weatherburn, D. C., 2005, Elemental analysis and source apportionment of ambient particulate matter at Masterton, New Zealand. International Journal of PIXE, 15(3/4), 225-231.

Davy, P., K. (2007). Composition and Sources of Aerosol in the Wellington Region of New Zealand. PhD Thesis, School of Chemical and Physical Sciences, Victoria University of Wellington, Wellington. 
Davy, P. K., Trompetter, W. J. and Markwitz, A. (2010). Source apportionment of airborne particles in the Auckland region: 2010 Analysis. Wellington, GNS Science Client Report 2010/262.

Davy, P., K., Trompetter, W. and Markwitz, A. (2011). Concentration, composition and sources of particulate matter in the Johnstone Hills Tunnel, Auckland, GNS Science Client Report 2010/296.

Davy, P. K., Ancelet, T., Trompetter, W. J., Markwitz, A. and Weatherburn, D. C., 2012, Composition and source contributions of air particulate matter pollution in a New Zealand suburban town. Atmospheric Pollution Research, 3, 143147.

Dockery, D. W., Pope III, C. A., Xu, X., Spengler, J. D., Ware, J. H., Fay, M. E., Ferris, B. G., Jr. and Speizer, F. E., 1993, An association between air pollution and mortality in six U.S. cities. New England Journal of Medicine, 329(24), 1753-1759.

Dye, A. L., Rhead, M. M. and Trier, C. J., 2000, The quantitative morphology of roadside and background urban aerosol in Plymouth, UK. Atmospheric Environment, 34(19), 3139-3148.

El Haddad, I., Marchand, N., Dron, J., Temime-Roussel, B., Quivet, E., Wortham, H., Jaffrezo, J. L., Baduel, C., Voisin, D., Besombes, J. L. and Gille, G., 2009, Comprehensive primary particulate organic characterization of vehicular exhaust emissions in France. Atmospheric Environment, 43(39), 6190-6198.

Epton, M. J., Dawson, R. D., Brooks, W. M., Kingham, S., Aberkane, T., Cavanagh, J.-A. E., Frampton, C. M., Hewitt, T., Cook, J. M., McLeod, S., McCartin, F., Trought, K. and Brown, L., 2008, The effect of ambient air pollution on respiratory health of school children: a panel study. Environmental Health, 7, DOI: 10.1186/1476-069X-7-16.

Falkovich, A. H. and Rudich, Y., 2001, Analysis of Semivolatile Organic Compounds in Atmospheric Aerosols by Direct Sample Introduction Thermal Desorption GC/MS. Environmental Science and Technology, 35(11), 2326-2333.

Fassel, V. A. and Kinseley, R. N., 1974, Inductively coupled plasma-optical emission spectroscopy. Analytical Chemistry, 46, 1110A-1120A.

Filippi, E., Prati, P., Zucchiatti, A., Lucarelli, F., Ariola, V. and Corvisiero, P., 1999, Hourly measurement of particulate concentrations with streaker samplers and 
optical methods. Nuclear Instruments and Methods in Physics Research, Section B: Beam Interactions with Materials and Atoms, 150(1-4), 370-374.

Fine, P. M., Cass, G. R. and Simoneit, B. R. T., 2002, Chemical characterzation of fine particle emissions from the fireplace combustion of woods grown in the Southern United States. Environmental Science and Technology, 36(7), 14421451.

Fisher, G. W., Rolfe, K. A., Kjellstrom, T., Woodward, A., Hales, S., Sturman, A. P., Kingham, S., Petersen, J., Shrestha, R. and King, D. (2002). Health effects due to motor vehicle air pollution in New Zealand, Report for the Ministry of Transport. URL: http://www.transport.govt.nz/research/Documents/healtheffects-of-vehicle-emissions.pdf.

Fisher, G. W., Kjellstrom, T., Kingham, S., Hales, S., Shrestha, R., Sturman, A., Sherman, M., O'Fallon, C., Cavanagh, J.-A. E. and Durand, M. (2007). Health and Air Pollution in New Zealand, Report for the Ministry for the Environment, the Ministry of Transport and the Health Research Council of New Zealand. URL: http://www.hapinz.org.nz.

Fisher, G., Barclay, J., Graham, B., Iremonger, S. and Thronton, D., 2008, Targeted air pollution reduction strategies using inventories and advanced modelling: Part 2 airshed modelling. Air Quality and Climate Change, 42, 25-29.

Fitzgerald, J. E., Skam, A. W. and Thom, N. G., 1973, Air pollution in Auckland: Now and in the future. Clean Air, 6-9.

Fitzgerald, J. W., 1991, Marine aerosols: A review. Atmospheric Environment, 25A (3-4), 533-545.

Fraser, M. P., Cass, G. R. and Simoneit, B. R. T., 1999, Particulate organic compounds emitted from motor vehicle exhaust and in the urban atmosphere. Atmospheric Environment, 33(17), 2715-2724.

Fraser, M. P., Lakshmanan, K., Fritz, S. G. and Ubanwa, B., 2002, Variation in composition of fine particulate emissions from heavy-duty diesel vehicles. Journal of Geophysical Research: Atmospheres, 107(D21), Article number 8346.

Friedlander, S. K., 1973, Chemical element balances and identification of air pollution sources. Environmental Science and Technology, 7, 235-240.

Fukuda, K., Hider, P. N., Epton, M. J., Jennings, L. C. and Kingham, S., 2011, Including viral infection data supports an association between particulate 
pollution and respiratory admissions. Australian and New Zealand Journal of Public Health, 35, 163-169.

Fung, K. K., 1990, Particulate carbon speciation by $\mathrm{MnO}_{2}$ oxidation. Aerosol Science and Technology, 12, 122-127.

Garg, B. D., Cadle, S. H., Mulawa, P. A., Groblicki, P. J., Laroo, C. and Parr, G. A., 2000, Brake wear particulate matter emissions. Environmental Science and Technology, 34, 4463-4469.

George, I. J. and Abbatt, J. P. D., 2010, Heterogeneous oxidation of atmospheric aerosol particles by gas-phase radicals. Nature Chemistry, 2, 713-722.

Gibbs, W. E. (1924). Clouds and smokes: The properties of disperse systems in gases and their practical applications. P. Blakiston's Son \& Co.: Philadelphia, PA.

Gillies, J. A. and Gertler, A. W., 2000, Comparison and evaluation of chemically speciated mobile source $\mathrm{PM}_{2.5}$ particulate matter profiles. Journal of the Air and Waste Management Association, 50(8), 1459-1480.

Gogou, A., Stratigakis, N., Kanakidou, M. and Stephanou, E. G., 1996, Organic aerosols in the Eastern Mediterranean: components source reconciliation by using molecular markers and atmospheric back trajectories. Organic Geochemistry, 25, 79-96.

Graham, B. and Iremonger, S., 2008, Targeted air pollution reduction strategies using inventories and advanced modelling: Part 1 emissions inventory. Air Quality and Climate Change, 42, 19-24.

Grahame, T. J. and Schlesinger, R. B., 2007, Do we know enough to consider regulating specific particle types or sources? Inhalation Toxicology, 19, 457481.

Gras, J., Meyer, C., Weeks, R., Gillett, R., Galbally, I., Todd, J., Carnovale, F., Joynt, R., Hinwood, A., Berko, H. and Brown, S., 2002, Emissions from domestic solid fuel burning appliances (wood-heaters, open fireplaces). Technical Report No. 5, Environment Australia.

Grieshop, A. P., Lipsky, E. M., Pekney, N. J., Takahama, S. and Robinson, A. L., 2006, Fine particle emission factors from vehicles in a highway tunnel: Effects of fleet composition and season. Atmospheric Environment, 40(Supplement 2), 287-298.

Gwinn, M. R. and Vallyathan, V., 2006, Nanoparticles: Health effects - Pros and cons. Environmental Health Perspectives, 114, 1818-1825. 
Hales, S., Blakely, T. and Woodward, A., 2010, Air pollution and mortality in New Zealand: cohort study. Journal of Epidemiology and Community Health, DOI: 10.1136/jech.2010.112490.

Hamilton, B., Spronken-Smith, R. and Zawar-Reza, P., 2004, Spatial patterns of particulate episodes in Rangiora and Kaiapoi, Canterbury, New Zealand. Air Quality and Climate Change, 38, 26-32.

Hannigan, M. P., Busby, W. F., Jr. and Cass, G. R., 2005, Source contributions to the mutagenicity of urban particulate air pollution. Journal of the Air and Waste Management Association, 55(4), 399-410.

Hays, M. D. and Lavrich, R. J., 2007, Developments in direct thermal extraction gas chromatography-mass spectrometry of fine aerosols. Trac-Trends in Analytical Chemistry, 26(2), 88-102.

Haywood, J. and Boucher, O., 2000, Estimates of the direct and indirect radiative forcing due to tropospheric aerosols: a review. Reviews of Geophysics, 38(4), 513-543.

Hedberg, E., Kristensson, A., Ohlsson, M., Johansson, C., Johansson, P.-A., Swietlicki, E., Vesely, V., Wideqvist, U. and Westerholm, R., 2002, Chemical and physical characterization of emissions from birch wood combustion in a wood stove. Atmospheric Environment, 36(30), 4823-4837.

Hildemann, L. M., Markowski, G. R. and Cass, G. R., 1991, Chemical composition of emissions from urban sources of fine organic aerosol. Environmental Science and Technology, 25(4), 744-759.

Hinds, W. C. 1999. Aerosol Technology. New York, Wiley.

Ho, K. F., Ho, S. S. H., Lee, S. C., Cheng, Y., Chow, J. C., Watson, J. G., Louie, P. K. K. and Tian, L., 2009, Emissions of gas- and particle-phase polycyclic aromatic hydrocarbons (PAHs) in the Shing Mun Tunnel, Hong Kong. Atmospheric Environment, 43(40), 6343-6351.

Hopke, P. K. (1999a). An introduction to source receptor modeling. Elemental analysis of airborne particles. Landsberger, S. and Creatchman, M., eds. Amsterdam, Gordon Breach Science Publications. 1: 273-316.

Hopke, P. K. (1999b). Quality assurance, quality control and data validation in environmental analysis of airborne particles. Elemental analysis of airborne particles. Landsberger, S. and Creatchman, M., eds. Amsterdam, Gordon and Breach Science Publishers. 1: 235-254. 
Horvath, H., 1993, Atmospheric Light Absorption - A Review. Atmospheric Environment, 27A, 293-317.

Horvath, H., 1997, Experimental calibration for aerosol light absorption measurements using the integrating plate method - Summary of the data. Aerosol Science, 28, 2885-2887.

Howden-Chapman, P., Pierse, N., Nicholls, S., Gillespie-Bennett, J., Viggers, H., Cunningham, M., Phipps, R., Boulic, M., Fjällström, P., Free, S., Chapman, R., Lloyd, B., Wickens, K., Shields, D., Baker, M., Cunningham, C., Woodward, A., Bullen, C. and Crane, J., 2008, Effects of improved home heating on asthma in community dwelling children: randomised controlled trial. BMJ, 337, DOI: 10.1163/bmj.a1411.

Huang, X. F., Yu, J. Z., He, L. Y. and Hu, M., 2006, Size distribution characteristics of elemental carbon emitted from Chinese vehicles: results of a tunnel study and atmospheric implications. Environmental Science and Technology, 40, 5355-5360.

Hung-Lung, C. and Yao-Sheng, H., 2009, Particulate matter emissions from on-road vehicles in a freeway tunnel study. Atmospheric Environment, 43(26), 40144022.

Hunt, A., Abraham, J. L., Judson, B. and Berry, C. L., 2003, Toxicologic and epidemiologic clues from the characterization of the 1952 London smog fine particulate matter in archival autopsy lung tissues. Environmental Health Perspectives, 111(9), 1209-1214.

Huntzicker, J. J., Johnson, R. L., Shah, J. J. and Cary, R. A. (1982). Analysis of organic and elemental carbon in ambient aerosol by a thermal-optical method. Atmospheric Life Cycle. Wolff, G. T. and Klimisch, R. L., eds. New York, NY, Plenum Press: 79-88.

IARC (1998). Polynuclear Aromatic Compounds, Part 1: Chemical, Environmental and Experimental Data. Air Pollution, Part 1, Vol. 92.

IPCC 2007a. Climate Change 2007: Synthesis Report. Geneva, Switzerland, IPCC.

IPCC 2007b. Contribution of Working Group I to the Fourth Assessment Report of the Intergovernmental Panel on Climate Change, 2007. Cambridge, United Kingdom and New York, NY, USA, Cambridge University Press. 
Jacobson, M. C., Hansson, H. C., Noone, K. J. and Charlson, R. J., 2000, Organic atmospheric aerosols: review and state of the science. Reviews of Geophysics, 38(2), 267-294.

Jimenez, J. L., Canagaratna, M. R., Donahue, N. M., Prevot, A. S. H., Zhang, Q., Kroll, J. H., DeCarlo, P. F., Allan, J. D., Coe, H., Ng, N. L., Aiken, A. C., Docherty, K. S., Ulbrich, I. M., Grieshop, A. P., Robinson, A. L., Duplissy, J., Smith, J. D., Wilson, K. R., Lanz, V. A., Hueglin, C., Sun, Y. L., Tian, J., Laaksonen, A., Raatikainen, T., Rautiainen, J., Vaattovaara, P., Ehn, M., Kulmala, M., Tomlinson, J. M., Collins, D. R., Cubison, M. J., Dunlea, E. J., Huffman, J. A., Onasch, T. B., Alfarra, M. R., Williams, P. I., Bower, K., Kondo, Y., Schneider, J., Drewnick, F., Borrmann, S., Weimer, S., Demerjian, K., Salcedo, D., Cottrell, L., Griffin, R., Takami, A., Miyoshi, T., Hatakeyama, S., Shimono, A., Sun, J. Y., Zhang, Y. M., Dzepina, K., Kimmel, J. R., Sueper, D., Jayne, J. T., Herndon, S. C., Trimborn, A. M., Williams, L. R., Wood, E. C., Middlebrook, A. M., Kolb, C. E., Baltensperger, U. and Worsnop, D. R., 2009, Evolution of organic aerosols in the atmosphere. Science, 326(5959), 1525-1529.

Jordan, T. B., Seen, A. J., Jacobsen, G. E. and Gras, J. L., 2006, Radiocarbon determination of woodsmoke contribution to air particulate matter in Launceston, Tasmania. Atmospheric Environment, 40, 2575-2582.

Junninen, H., Mønster, J., Rey, M., Cancelinha, J., Douglas, K., Duane, M., Forcina, V., Müller, A., Lagler, F., Marelli, L., Borowiak, A., Niedzialek, J., Paradiz, B., Mira-Salama, D., Jimenez, J., Hansen, U., Astorga, C., Stanczyk, K., Viana, M., Querol, X., Duvall, R. M., Norris, G. A., Tsakovski, S., Wåhlin, P., Horák, J. and Larsen, B. R., 2009, Quantifying the Impact of Residential Heating on the Urban Air Quality in a Typical European Coal Combustion Region. Environmental Science and Technology, 43(20), 7964-7970.

Kelly, C., Mues, S. and Webley, W., 2007, Real-life Emissions Testing of Wood Burners in Tokoroa. Ministry for the Environment Warm Homes report number ME814.

Keywood, M. D., Ayers, G. P., Gras, J. L., Gillett, R. W. and Cohen, D. D., 2000, Size distribution and sources of aerosol in Launceston, Australia, during winter 1997. Journal of the Air and Waste Management Association, 50(3), 418-427. 
Khalil, M. A. K. and Rasmussen, R. A., 2003, Tracers of wood smoke. Atmospheric Environment, 37(9-10), 1211-1222.

Khanal, O. R. and Shooter, D., 2004, Benzo(a)pyrene in atmospheric particles from three New Zealand cities: Variations between cities and seasons. Air Quality and Climate Change, 38, 28-31.

Kim, E. and Hopke, P. K., 2007, Source identification of airborne fine particles using PMF2 and EPA PMF. Journal of the Air and Waste Management Association, 57, 811-819.

Kim, E. and Hopke, P. K., 2008, Source characterization of ambient fine particles at multiple sites in the Seattle area. Atmospheric Environment, 42(24), 60476056.

Kleeman, M. J., Schauer, J. J. and Cass, G. R., 1999, Size and Composition Distribution of Fine Particulate Matter Emitted from Wood Burning, Meat Charbroiling, and Cigarettes. Environmental Science and Technology, 33(20), 3516-3523.

Klemm, R., Mason, R., Heilig, C. and Cowan, D., 2000, The effect of imputation of exposure estimates on the association between fine particulate matter and mortality. Annals of Epidemiology, 10(7), 477-478.

Kocbach, A., Johansen, B. V., Schwarze, P. E. and Namork, E., 2005, Analytical electron microscopy of combustion particles: a comparison of vehicle exhaust and residential wood smoke. Science of the Total Environment, 346(1-3), 231243.

Kossmann, M. and Sturman, A., 2004, The surface wind field during winter smog nights in Christchurch and coastal Canterbury, New Zealand. International Journal of Climatology, 24, 93-108.

Kristensson, A., Johansson, C., Westerholm, R., Swietlicki, E., Gidhagen, L., Wideqvist, U. and Vesely, V., 2004, Real-world traffic emission factors of gases and particles measured in a road tunnel in Stockholm, Sweden. Atmospheric Environment, 38(5), 657-673.

Krivácsy, Z., Blazsó, M. and Shooter, D., 2006, Primary organic pollutants in New Zealand urban aerosol in winter during high PM10 episodes. Environmental Pollution, 139(2), 195-205. 
Kuschel, G. and Mahon, K. (2010). A Review and Update of HAPiNZ for the Auckland Region. Internal report no. 2010/004 to Auckland Regional Council. Auckland.

Landsberger, S. and Creatchman, M. 1999. Elemental Analysis of Airborne Particles. [In: Advances in Environmental, Industrial and Process Control Technologies, 1999; 1$]$.

Lavin, K. S., Hageman, K. J., Marx, S. K., Dillingham, P. W. and Kamber, B. S., 2012, Using trace elements in particulate matter to identify the sources of semivolatile orgnaic contaminants in air at an alpine site. Environmental Science and Technology, 46, 268-276.

Lee, R. G. M., Coleman, P., Jones, J. L., Jones, K. C. and Lohmann, R., 2005, Emission factors and importance of PCDD/Fs, PCBs, PCNs, PAHs and PM10 from the domestic burning of coal and wood in the UK. Environmental Science and Technology, 39(6), 1436-1447.

Lewtas, J., 1993, Complex mixtures of air pollutants: characterizing the cancer risk of polycyclic organic matter. Environmental Health Perspectives, 100, 211-218.

Li, J., Posfai, M., Hobbs, P. V. and Buseck, P. R., 2003, Individual aerosol particles from biomass burning in southern Africa: 2. Compositions and aging of inorganic particles. J. Geophys. Res. Atmos., 108(D13), Article number 8484.

Lighty, J. S., Veranth, J. M. and Sarofim, A. F., 2000, Combustion aerosols: factors governing their size and composition and implications to human health. Journal of the Air and Waste Management Association, 50(9), 1565-1618; discussion 1619-1622.

Lima, A. L. C., Farrington, J. W. and Reddy, C. M., 2005, Combustion-derived polycyclic aromatic hydrocarbons in the environment - A review. Environmental Forensics, 6(2), 109-131.

Longley, I. and Gadd, J., 2011, Preliminary exploration of high resolution PM10 measured inside wood-burning New Zealand homes. Air Quality and Climate Change, 45, 21-26.

Longley, I. D. and Harper, S., 2010, Interpreting the influence of an urban valley micro-climate on PM10 concentrations from monitoring data. Air Quality and Climate Change, 44, 15-21. 
Lucarelli, F., Nava, S., Calzolai, G., Chiari, M., Udisti, R. and Marino, F., 2011, Is PIXE still a useful technique for the analysis of atmospheric aerosols? The LABEC experience. X-Ray Spectrometry, 40, 162-167.

MacCarthy, J., Thomas, J., Choudrie, S., Passant, N., Thistlewaite, G., Murrells, T., Watterson, J., Cardenas, L. and Thomson, A. (2010). UK Greenhouse Gas Inventory, 1990 to 2008. Oxfordshire, UK.

Mader, B. T., Flagan, R. C. and Seinfeld, J. H., 2001, Sampling atmospheric carbonaceous aerosols using a particle trap impactor/denuder sampler. Environmental Science and Technology, 35, 4857-4867.

Maenhaut, W., Francois, F. and Cafmeyer, J. (1993). The “Gent” Stacked Filter Unit Sampler for the Collection of Atmospheric Aerosols in Two Size Fractions: Description and Instructions for Installation and Use. Co-ordinated Research Programme: CRP E4.10.08. Vienna, International Atomic Energy Agency.

Maenhaut, W. and Malmqvist, K., G. (2001). Particle Induced X-ray Emission Analysis. Handbook of x-ray spectrometry. Grieken, R. V. ed. Antwerp, Marcel Dekker Inc.

Malm, W. C. and Day, D. E., 2000, Optical properties of aerosols at Grand Canyon National Park. Atmospheric Environment, 34(20), 3373-3391.

Malm, W. C., Sisler, J. F., Huffman, D., Eldred, R. A. and Cahill, T. A., 1994, Spatial and seasonal trends in particle concentration and optical extinction in the United States. Journal of Geophysical Research: Atmospheres, 99(D1), 13471370.

Manins, P. C. and Sawford, B. L., 1979, A model of katabatic winds. Journal of the Atmospheric Sciences, 36, 619-630.

Mauderly, J. L., Chow, J. C., 2008, Health effects of organic aerosols. Inhalation Toxicology, 20(3), 257-288.

Mazzei, F., Lucarelli, F., Nava, S., Prati, P., Valli, G. and Vecchi, R., 2007, A new methodological approach: The combined use of two-stage streaker samplers and optical particle counters for the characterization of airborne particulate matter. Atmospheric Environment, 41(26), 5525-5535.

McConnell, R., Berhane, K., Yao, L., Jerrett, M., Lurmannn, F., Gilliland, F., Kunzli, N., Gauderman, J., Avol, E., Thomas, D. and Peters, J., 2006, Traffic, susceptibility, and childhood asthma. Environmental Health Perspectives, 114, 766-772. 
McDonald, J. D., Zielinska, B., Fujita, E. M., Sagebiel, J. C., Chow, J. C. and Watson, J. G., 2000, Fine Particle and Gaseous Emission Rates from Residential Wood Combustion. Environmental Science and Technology, 34(11), 2080-2091.

McGowan, J. A., Hider, R. N., Chacko, E. and Town, G. I., 2002, Particulate air pollution and hospital admissions in Christchurch, New Zealand. Australian and New Zealand Journal of Public Health, 26(1), 23-29.

McKendry, I. G., Sturman, A. P. and Vergeiner, J., 2004, Vertical profiles of particulate matter size distributions during winter domestic burning in Christchurch, New Zealand. Atmospheric Environment, 38(29), 4805-4813.

Menichini, E., 1992, Urban air pollution by polycyclic aromatic hydrocarbons: levels and sources of variability. Science of the Total Environment, 116(1-2), 109135.

Ministry for the Environment (2001). Emissions Inventories for New Zealand. Wellington: Ministry for the Environment.

Ministry for the Environment (2002a), Ambient Air Quality Guidlines. Air Quality Report No. 32, Ministry for the Environment.

Ministry for the Environment (2002b). New Zealand Ambient Air Quality Guidelines. Wellington: Ministry for the Environment.

Ministry for the Environment (2011). 2011 User's Guide to the revised National Environmental Standards for Air Quality 2011. Wellington: Ministry for the Environment.

Mkoma, S. L., Chi, X. and Maenhaut, W., 2010, Characteristics of carbonaceous aerosols in ambient PM10 and PM2.5 particles in Dar es Salaam, Tanzania. Science of the Total Environment, 408, 1308-1314.

Mobley, J., D. and Cadle, S., 2004, Innovative methods for emissions inventory development and evaluation: workshop summary. Journal of the Air and Waste Management Association, 54, 1422-1439.

Moreno, T., Gibbons, W., Jones, T. and Richards, R., 2003, The geology of ambient aerosols: characterising urban and rural/coastal silicate PM10-2.5 and PM2..5 using high-volume cascade collection and scanning electron microscopy. Atmospheric Environment, 37(30), 4265-4276.

Murdoch, D. R. and Jennings, L. C., 2009, Association of respiratory virus activity and environmental factors with the incidence of pneumococcal disease. Journal of Infection, 58, 37-46. 
Nel, A., 2005, Air pollution-related illness: effects of particles. Science, 308(5723), 804-806.

Okuda, T., Kumata, H., Naraoka, H. and Takada, H., 2002a, Origin of atmospheric polycyclic aromatic hydrocarbons (PAHs) in Chinese cities solved by compound-specific stable carbon isotopic analyses. Organic Geochemistry, 33(12), 1737-1745.

Okuda, T., Kumata, H., Zakaria, M. P., Naraoka, H., Ishiwatari, R. and Takada, H., 2002b, Source identification of Malaysian atmospheric polycyclic aromatic hydrocarbons nearby forest fires using molecular and isotopic compositions. Atmospheric Environment, 36, 611-618.

O'Malley, V. P., Abrajano Jr., T. A. and Hellou, J., 1994, Determination of the ${ }^{13} \mathrm{C} /{ }^{12} \mathrm{C}$ of individual PAH from environmental samples: can PAH sources be apportioned? Organic Geochemistry, 21, 809-822.

Paatero, P. and Tapper, U., 1993, Analysis of different modes of factor analysis as least squares fit problems. Chemometrics and Intelligent Laboratory Systems, 18, 183-194.

Paatero, P. and Tapper, U., 1994, Positive matrix factorization: a non-negative factor model with optimal utilization of error estimates of data values. Environmetrics, 5(2), 111-126.

Paatero, P., 1997, Least squares formulation of robust non-negative factor analysis. Chemometrics and Intelligent Laboratory Systems, 18, 183-194.

Paatero, P., 1999, The multilinear engine - a table driven, least square program for solving multilinear problems, including the n-way parallel factor analysis model. Journal of Computational and Graphical Statistics, 8, 854-888.

Paatero, P., Hopke, P. K., Song, X. H. and Ramadan, Z., 2002, Understanding and controlling rotations in factor analytic models. Chemometrics and Intelligent Laboratory Systems, 60(1-2), 253-264.

Paatero, P. and Hopke, P. K., 2003, Discarding or downweighting high-noise variables in factor analytic models. Analytica Chimica Acta, 490(1-2), 277289.

Paatero, P., Hopke, P. K., Begum, B. A. and Biswas, S. K., 2005, A graphical diagnostic method for assessing the rotation in factor analytical models of atmospheric pollution. Atmospheric Environment, 39(1), 193-201. 
Paoletti, L., Diociaiuti, M., De Berardis, B., Santucci, S., Lozzi, L. and Picozzi, P., 1999, Characterisation of aerosol individual particles in a controlled underground area. Atmospheric Environment, 33(22), 3603-3611.

Pearce, J. and Kingham, S., 2008, Environmental inequalities in New Zealand: A national study of air pollution and environmental justice. Geoforum, 39, 980993.

Peré-Trepat, E., Kim, E., Paatero, P. and Hopke, P. K., 2007, Source apportionment of time and size resolved ambient particulate matter measured with a rotating DRUM impactor. Atmospheric Environment, 41(28), 5921-5933.

Petersen, J. and Fisher, G. (1999). Elemental compositions of particulates in Hamilton-Preliminary study. NIWA Report AK98113.

Polissar, A. V., Hopke, P. K., Paatero, P., Malm, W. C. and Sisler, J. F., 1998, Atmospheric aerosol over Alaska 2. Elemental composition and sources. Journal of Geophysical Research: Atmospheres, 103(D15), 19045-19057.

Pope, C. A., 3rd, 1989, Respiratory disease associated with community air pollution and a steel mill, Utah Valley. American Journal of Public Health, 79(5), 623628.

Pope, C. A., 3rd, 1996, Adverse health effects of air pollutants in a nonsmoking population. Toxicology, 111(1-3), 149-155.

Pope, C. A., 3rd, Dockery, D. W., 2006, Critical review: Health effects of fine particulate air pollution: Lines that connect. Journal of the Air and Waste Management Association, 56(6), 709-742.

Pöschl, U., 2005, Atmospheric Aerosols: Composition, Transformation, Climate and Health Effects. Angewandte Chemie International Edition, 44(46), 7520-7540.

Pósfai, M. and Buseck, P. R., 2010, Nature and climate effects of individual tropospheric aerosol particles. Annual Reviews of Earth and Planetary Sciences, 38, 17-43.

Prati, P., Zucchiatti, A., Lucarelli, F. and Mando, P. A., 2000, Source apportionment near a steel plant in Genoa (Italy) by continuous aerosol sampling and PIXE analysis. Atmospheric Environment, 34(19), 3149-3157.

Qin, Y., Kim, E. and Hopke, P. K., 2006, The concentrations and sources of PM2.5 in metropolitan New York City. Atmospheric Environment, 40 (Supplement 2), 312-332. 
R Development Core Team (2011). R: A language and environment for statistical computing. R Foundation for Statistical Computing, Vienna, Austria.

Raes, F., Dingenen, R. V., Vignati, E., Wilson, J., Putaud, J.-P., Seinfeld, J. H. and Adams, P., 2000, Formation and cycling of aerosols in the global troposphere. Atmospheric Environment, 34(25), 4215-4240.

Ramdahl, T., 1983, Retene - a molecular marker of wood combustion in ambient air. Nature, 306(5943), 580-582.

Reisinger, A. R., 2000, Observations of $\mathrm{HNO}_{2}$ in the polluted winter atmosphere: possible heterogeneous production on aerosols. Atmospheric Environment, 34(23), 3865-3874.

Rogge, W. F., Hildemann, L. M., Mazurek, M. A., Cass, G. R. and Simoneit, B. R. T., 1998, Sources of Fine Organic Aerosol. 9. Pine, Oak, and Synthetic Log Combustion in Residential Fireplaces. Environmental Science and Technology, 32(1), 13-22.

Russell, A. G. and Brunekreef, B., 2009, A focus on particulate matter and health. Environmental Science and Technology, 43(13), 4620-4625.

Rybicki, B. A., Nock, N. L., Savera, A. T., Tang, D. and Rundle, A., 2006, Polycyclic aromatic hydrocarbon-DNA adduct formation in prostate carcinogenesis. Cancer Letters, 239(2), 157-167.

Salma, I., Chi, X. and Maenhaut, W., 2004, Elemental and organic carbon in urban canyon and background environments in Budapest, Hungary. Atmospheric Environment, 38(1), 27-36.

Schauer, J. J., Kleeman, M. J., Cass, G. R. and Simoneit, B. R. T., 1999, Measurement of Emissions from Air Pollution Sources. 2. C1 through C30 Organic Compounds from Medium Duty Diesel Trucks. Environmental Science and Technology, 33(10), 1578-1587.

Schauer, J. J., Kleeman, M. J., Cass, G. R. and Simoneit, B. R., 2001, Measurement of emissions from air pollution sources. 3. C1-C29 organic compounds from fireplace combustion of wood. Environmental Science and Technology, 35(9), 1716-1728.

Schauer, J. J., Kleeman, M. J., Cass, G. R. and Simoneit, B. R. T., 2002, Measurement of Emissions from Air Pollution Sources. 5. C1-C32 Organic Compounds from Gasoline-Powered Motor Vehicles. Environmental Science and Technology, 36(6), 1169-1180. 
Schauer, J. J., Lough, G. C., Shafer, M. M., Christensen, W. F., Arndt, M. F., Deminter, J. T. and Park, J.-S., 2006, Characterisation of metals emitted from motor vehicles. Research Report 133. Health Effects Institute, Boston.

Schwartz, J., 1991, Particulate air pollution and daily mortality: a synthesis. Public Health Reviews, 19(1-4), 39-60.

Schwartz, J., 1996, Air pollution and hospital admissions for respiratory disease. Epidemiology, 7(1), 20-28.

Scott, A. J. (2005). Real-life emissions from residential wood burning appliances in New Zealand. Christchurch, Ministry for the Environment.

Scott, A. J. (2006). Source Apportionment and Chemical Characterisation of Airborne Fine Particulate Matter in Christchurch, New Zealand. PhD Thesis, University of Canterbury. Christchurch.

Scott, A. J. and Scarrott, C., 2011, Impacts of residential heating intervention measures on air quality and progress towards targets in Christchurch and Timaru, New Zealand. Atmospheric Environment, 45(17), 2972-2980.

Seinfeld, J. H., 2004, Air pollution: A half century of progress. Aiche Journal, 50, 1096-1108.

Seinfeld, J. H. and Pandis, S. N. 2006. Atmospheric Chemistry and Physics: From Air Pollution to Climate Change. New York, John Wiley \& Sons, Inc.

Senaratne, I. and Shooter, D., 2004, Elemental composition in source identification of brown haze in Auckland, New Zealand. Atmospheric Environment, 38(19), 3049-3059.

Senaratne, I., Kelliher, F. M. and Triggs, C. M., 2005, Source apportionment of PM10 during cold, calm weather in Christchurch, New Zealand: Preliminary results from a receptor model. Air Quality and Climate Change, 39, 47-54.

Simoneit, B. R. T., Schauer, J. J., Nolte, C. G., Oros, D. R., Elias, V. O., Fraser, M. P., Rogge, W. F. and Cass, G. R., 1998, Levoglucosan, a tracer for cellulose in biomass burning and atmospheric particles. Atmospheric Environment, 33(2), 173-182.

Simoneit, B. R. T., 1999, A review of biomarker compounds as source indicators and tracers for air pollution. Environmental Science and Pollution Research International, 6(3), 159-169.

Simoneit, B. R. T., 2002, Biomass burning - A review of organic tracers for smoke from incomplete combustion. Applied Geochemistry, 17(3), 129-162. 
Smith, J., Bluett, J., Wilton, E. and Mallet, T., 2008, In home testing of particulate emissions from NES-authorised woodburners: Nelson, Rotorua and Taumaranui 2007. NIWA Client Report: CHC2008-092.

Solomon, S., 1999, Stratospheric ozone depletion: A review of concepts and history. Reviews of Geophysics, 37, 275-316.

Song, X. H., Polissar, A. V. and Hopke, P. K., 2001, Sources of fine particle composition in the northeastern US. Atmospheric Environment, 35(31), 52775286.

Sparrow, C. J., 1968, Some geographical aspects of air pollution in Auckland. Clean Air, 22, 4.

Sparrow, C. J., Skam, A. W. and Thom, N. G., 1969, The growth and work of the Auckland Air Pollution Reseach Committee. Clean Air, 3-12.

Spronken-Smith, R. A., Sturman, A. P. and Wilton, E., 2001, The air pollution problem in Christchurch, New Zealand - Progress and prospects. Clean Air and Environmental Quality, 36, 23-28.

Staniswalis, J. G., Parks, N. J., Bader, J. O. and Maldonado, Y. M., 2005, Temporal analysis of airborne particulate matter reveals a dose-rate effect on mortality in El Paso: indications of differential toxicity for different particle mixtures. Journal of the Air and Waste Management Association, 55(7), 893-902.

Statistics New Zealand, 2006, Census 2006.

Stone, R., 2002, Air pollution - Counting the cost of London's killer smog. Science, 298, 2106-2107.

Sturman, A. P. and Zawar-Reza, P., 2002, Application of back trajectory techniques to the delimitation of urban clean air zones. Atmospheric Environment, 36, 3339-3350.

Tate, A. and Spronken-Smith, R. A., 2008, A preliminary investigation of the air pollution meteorology of Alexandra, central Otago, New Zealand. Air Quality and Climate Change, 42, 15-21.

Tate, A., Cullen, N. J. and Spronken-Smith, R. A., 2011, Wintertime PM10 measurements and modelling in Alexandra, central Otago, New Zealand: Understanding the bi-modal peak in evening air pollution. Air Quality and Climate Change, 45, 19-25. 
Thorpe, A. and Harrison, R. M., 2008, Sources and properties of non-exhaust particulate matter from road traffic: A review. Science of the Total Environment, 400(1-3), 270-282.

Todd, J. J., 2008, Emission auditing of new woodheaters in Australia and New Zealand. Air Quality and Climate Change, 42, 15-17.

Trompetter, W., Markwitz, A. and Davy, P., K., 2005, Air particulate research capability at the New Zealand Ion Beam Analysis Facility using PIXE and IBA Techniques. International Journal of PIXE, 15(3\&4), 249-255.

Trompetter, W. J., Davy, P. K. and Markwitz, A., 2010, Influence of environmental conditions on carbonaceous particle concentrations within New Zealand. Journal of Aerosol Science, 41(1), 134-142.

Tsai, S. S., Huang, C. H., Goggins, W. B., Wu, T. N. and Yang, C. Y., 2003, Relationship between air pollution and daily mortality in a tropical city: Kaohsiung, Taiwan. Journal of Toxicology and Environmental Health: A, 66(14), 1341-1349.

Tsai, Y. I. and Cheng, M. T., 1999, Visibility and aerosol chemical compositions near the coastal area in Central Taiwan. Science of the Total Environment, 231(1), 37-51.

Turpin, B. J., Saxena, P. and Andrews, E., 2000, Measuring and simulating particulate organics in the atmosphere: problems and prospects. Atmospheric Environment, 34, 2983-3013.

Twomey, S., 1974, Pollution and planetary albedo. Atmospheric Environment, 8, 1251-1256.

Twomey, S., 1977, Influence of pollution on shortwave albedo of clouds. Journal of the Atmospheric Sciences, 34, 1149-1152.

USEPA (1999). Compendium of Methods for the Determination of Inorganic Compounds in Ambient Air. United States Environment Protection Agency.

USEPA (2008). EPA Positive Matrix Factorization (PMF) 3.0 Fundamentals and User Guide. National Exposure Research Laboratory, Research Triangle Park, NC.

USEPA. (2009). "National Emissions Inventory (NEI) Air Pollutant Emissions Trend Data. URL: http://www.epa.gov/ttnchie1/trends/.

Viana, M., Chi, X., Maenhaut, W., Querol, X., Alastuey, A., Mikuska, P. and Vecera, Z., 2006, Organic and elemental carbon concentrations in carbonaceous 
aerosols during summer and winter sampling campaigns in Barcelona, Spain. Atmospheric Environment, 40(12), 2180-2193.

Viana, M., Maenhaut, W., ten Brink, H. M., Chi, X., Weijers, E., Querol, X., Alastuey, A., Mikuska, P. and Vecera, Z., 2007, Comparative analysis of organic and elemental carbon concentrations in carbonaceous aerosols in three European cities. Atmospheric Environment, 41(28), 5972-5983.

Vedal, S., 1997, Critical review - Ambient particles and health: Lines that divide. Journal of the Air and Waste Management Association, 47(5), 551-581.

Wang, H. and Shooter, D., 2001, Water soluble ions of atmospheric aerosols in three New Zealand cities: seasonal changes and sources. Atmospheric Environment, 35(34), 6031-6040.

Wang, H. and Shooter, D., 2002, Coarse-fine and day-night differences of watersoluble ions in atmospheric aerosols collected in Christchurch and Auckland, New Zealand. Atmospheric Environment, 36(21), 3519-3529.

Wang, H. and Shooter, D., 2005, Source apportionment of fine and coarse atmospheric particles in Auckland, New Zealand. Science of the Total Environment, 340(1-3), 189-198.

Wang, H., Kawamura, K. and Shooter, D., 2005a, Carbonaceous and ionic components in wintertime atmospheric aerosols from two New Zealand cities: Implications for solid fuel combustion. Atmospheric Environment, 39, 58655875.

Wang, H., Kawamura, K. and Shooter, D., 2005b, Carbonaceous and ionic components in wintertime atmospheric aerosols from two New Zealand cities: Implications for solid fuel combustion. Atmospheric Environment, 39(32), 5865-5875.

Wang, H., Kawamura, K. and Shooter, D., 2006, Wintertime organic aerosols in Christchurch and Auckland, New Zealand: Contributions of residential wood and coal burning and petroleum utilization. Environmental Science and Technology, 40(17), 5257-5262.

Ward, T. and Lange, T., 2010, The impact of wood smoke on ambient PM2.5 in northern Rocky Mountain valley communities. Environmental Pollution, 158(3), 723-729.

Waterman, D., Horsfield, B., Leistner, F., Hall, K. and Smith, S., 2000, Quantification of Polycyclic Aromatic Hydrocarbons in the NIST Standard Reference 
Material (SRM1649A) Urban Dust Using Thermal Desorption GC/MS. Analytical Chemistry, 72(15), 3563-3567.

Watson, J. G., 1984, Overview of receptor model principles. Journal of the Air Pollution Control Organization, 34(6), 619-623.

Watson, J. G., Cooper, J. A. and Huntzicker, J. J., 1984, The effective variance weighting for least squares calculations applied to the mass balance receptor model. Atmospheric Environment, 18(7), 1347-1355.

Watson, J., G., Chow, J., C. and Frazier, C. (1999). X-ray Fluorescence Analysis of Ambient Air Samples. Elemental analysis of airborne particles. Landsberger, S. and Creatchman, M, eds. Amsterdam, Gordon and Breach Science Publishers. 1: 67-96.

Watson, J. G., Chow, J. C. and Houck, J. E., 2001, PM2.5 chemical source profiles for vehicle exhaust, vegetative burning, geological material, and coal burning in Northwestern Colorado during 1995. Chemosphere, 43(8), 1141-1151.

Watson, J. G., 2002a, Visibility: Science and regulation - 2002 Critical review. Journal of the Air and Waste Management Association, 52(6), 628-713.

Watson, J. G., Zhu, T., Chow, J. C., Engelbrecht, J., Fujita, E. M. and Wilson, W. E., 2002b, Receptor modeling application framework for particle source apportionment. Chemosphere, 49(9), 1093-1136.

Whitby, K. T., Husar, R. B., Liu, B. Y. H. (1972a). The aerosol size distribution of Los Angeles smog. In Aerosols and Atmospheric Chemistry, Hidy, G. M., Ed.; Academic Press: New York, 237-264.

Whitby, K. T., Liu, B. Y. H., Husar, R. B., Barsic, N. J. (1972b). The Minnesota aerosol-analyzing system used in the Los Angeles smog project. In Aerosols and Atmospheric Chemistry, Hidy, G. M., Ed.; Academic Press: New York, 189-217.

Whitby, K. T., 1978, The physical characteristics of sulfur aerosols. Atmospheric Environment, 12, 135-139.

Whytlaw-Gray, R., Speakman, J. B., Campbell, J. H. P., 1923, Smokes part I - a study of their behaviour and a method of determining the number of particles they contain. Proceedings of the Royal Society London: A, 102, 600-615.

Widory, D., 2006, Combustibles, fuels and their combustion products: a view through carbon isotopes. Combustion Theory and Modelling, 10, 831-841. 
Williams, J., de Reus, M., Krejci, R., Fischer, H. and Ström, J., 2002, Application of the variability-size relationship to atmospheric aerosol studies: estimating aerosol lifetimes and ages. Atmospheric Chemistry and Physics, 2, 133-145.

Wilson, J. G., Kingham, S. and Sturman, A. P., 2006, Intraurban variations of PM10 air pollution in Christchurch, New Zealand: Implications for epidemiological studies. Science of the Total Environment, 367(2-3), 559-572.

Wilson, W. E., Chow, J. C., Claiborn, C., Fusheng, W., Engelbrecht, J. and Watson, J. G., 2002, Monitoring of particulate matter outdoors. Chemosphere, 49(9), 1009-43.

Wilton, E. (2003). Air Quality in Christchurch: An assessment of factors contirbuting to visibility degradation. PhD Thesis, University of Canterbury. Christchurch.

Wilton, E., Smith, J., Gimson, N. and Phillips, J., 2009, Source apportionment and modelling of PM10 in Hastings, New Zealand. Air Quality and Climate Change, 43, 31-39.

Xie, S. and Mahon, K. (2010). Effects of Fuel and Operation on Particulate Emissions from Woodburners. Auckland Regional Council Technical Repot 2010/048.

Zhu, C.-S., Chen, C.-C., Cao, J.-J., Tsai, C.-J., Chou, C. C. K., Liu, S.-C. and Roam, G.-D., 2010, Characterization of carbon fractions for atmospheric fine particles and nanoparticles in a highway tunnel. Atmospheric Environment, 44(23), 2668-2673.

Zielinska, B., Sagebiel, J., McDonald, J. D., Whitney, K. and Lawson, D. R., 2004, Emission rates and comparative chemical composition from selected in-use diesel and gasoline-fueled vehicles. Journal of the Air and Waste Management Association, 9, 1138-1150. 


\section{Appendix A. Analysis of Streaker filters by IBA}

The Ion Beam Technologies Department at the National Isotope Centre of GNS Science (Institute of Geological and Nuclear Sciences) has a long history of performing IBA on PM samples (Trompetter et al. 2005). Building on established IBA capabilities, a new protocol for analyzing Streaker filters was developed. This Appendix provides an overview of the developed protocol.

The main analysis technique used was PIXE using a $2.5 \mathrm{MV}$ proton beam produced by the GNS Science 3MV Ven de Graaff accelerator. A Si(Li) detector with a $25 \mu \mathrm{m}$ Be filter was used to measure X-rays produced as a result of the proton bombardment. The X-ray spectra produced were analyzed using the Gupix program (Maxwell et al. 1995), which de-convolves the overlapping X-ray peaks and background radiation. For light elements (eg. Na), PIGE analysis was used. The $\gamma-$ rays emitted during this analysis were detected with a $75 \mathrm{~mm}$ diameter HPGe detector mounted at $45^{\circ}$ in the forward direction from the sample. Figure A1 presents a schematic diagram of the measurement chamber detector layout and a schematic illustration of the beam line.

The procedures described in this Appendix were optimized to ensure that the filters were not damaged by the proton beam (eg. repeat measurements on the same spot showed no differences), the LODs obtained from the analyses were as low as possible and the sensitivity was as high as possible. Importantly, the process was streamlined as much as possible to ensure effective time management.

Prior to analysis of the Streaker filters, a number of analytical standards were measured. These standards were: $\mathrm{Al}\left(40.0 \mathrm{~g} \mathrm{~cm}^{-2}\right), \mathrm{SiO}\left(44.1 \mathrm{~g} \mathrm{~cm}^{-2}\right), \mathrm{KCl}(44.7 \mathrm{~g}$ $\mathrm{cm}^{-2}$ ), $\mathrm{Cr}\left(44.8 \mathrm{~g} \mathrm{~cm}^{-2}\right)$, Ni (39.0 and $\left.47.3 \mathrm{~g} \mathrm{~cm}^{-2}\right), \mathrm{SrF}_{2}\left(47.5 \mathrm{~g} \mathrm{~cm}^{-2}\right), \mathrm{NaCl}(10.5$, 15.8, 41.9 and $46 \mathrm{~g} \mathrm{~cm}^{-2}$ ). Each standard was run a number of times (6-7) to ensure consistency and standards were also analyzed periodically throughout the analysis of the Streaker filters. 


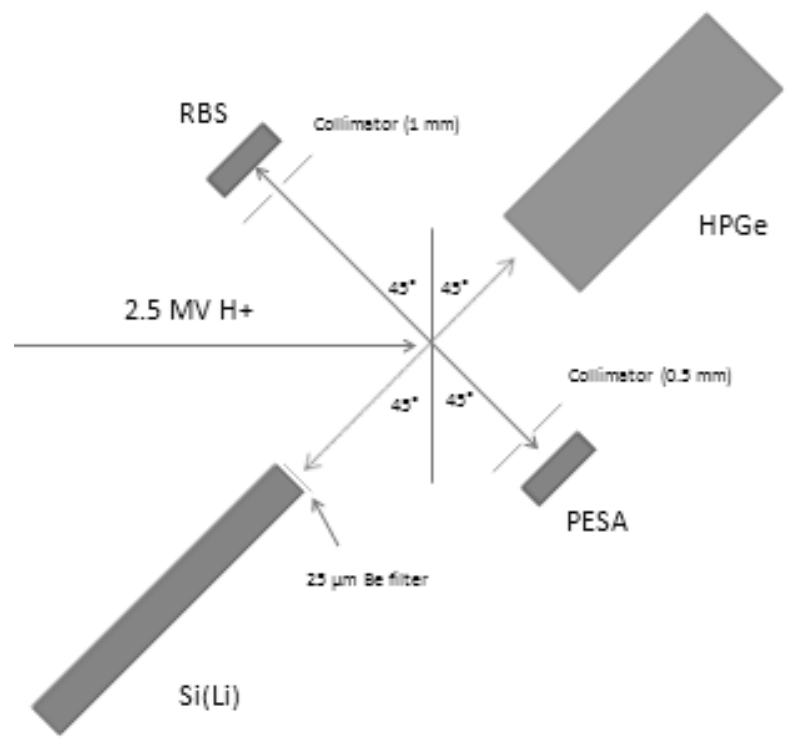

(a)

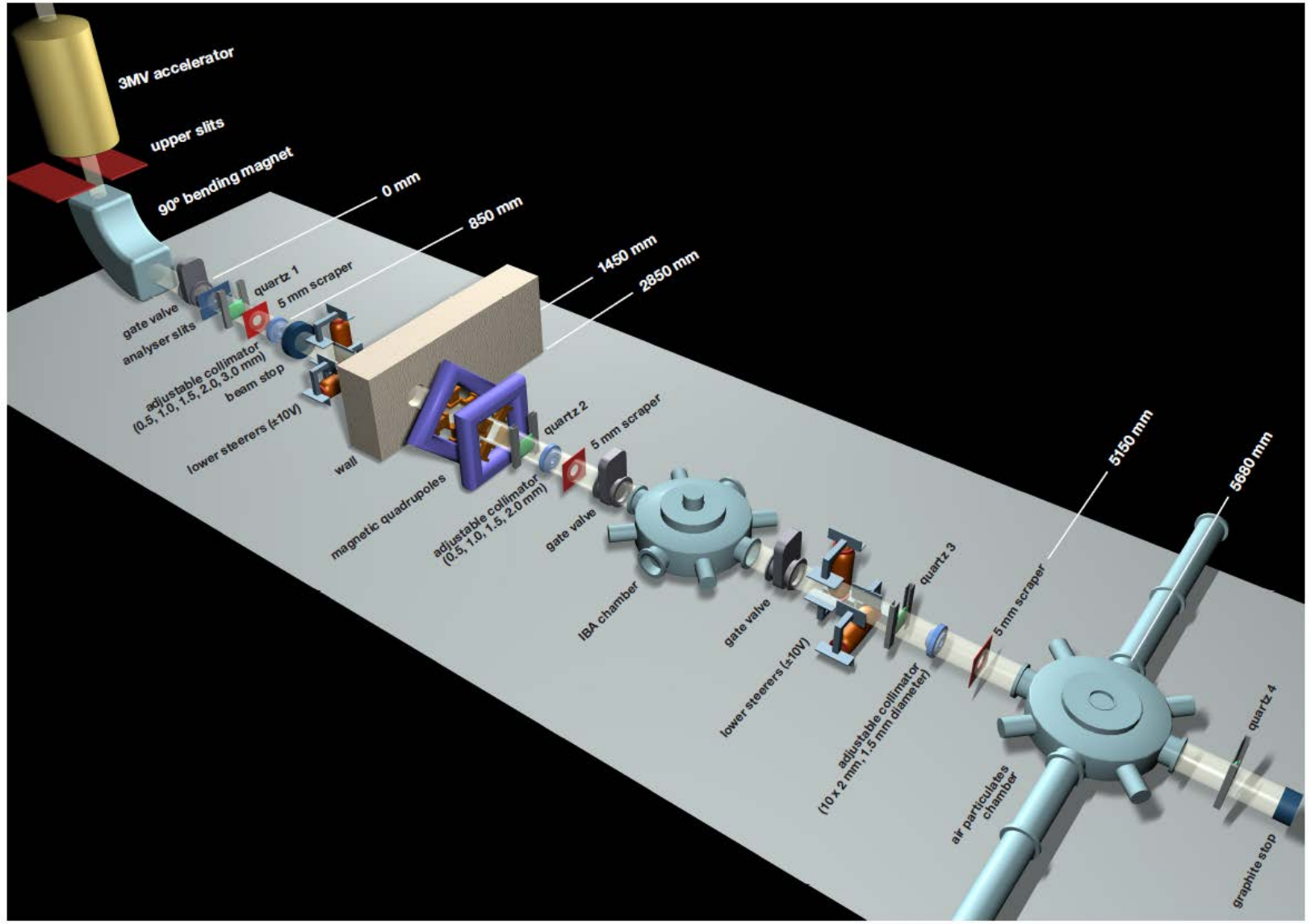

(b)

Figure A.1. Schematic illustration of the air particulate chamber detector layout (PIXE: particleinduced x-ray emission; PESA: particle elastic scattering; HPGE(PIGE): particle-induced gamma ray emission; RBS: Rutherford backscattering) (a) and a schematic illustration of the beam line (b).

To analyze the Streaker filters, the same stepper motor used in the Streaker sampler was mounted inside the air particulates chamber. This allowed exposed 
Streaker filters to be loaded and stepped for IBA in the same way that the samples were collected. An LED light was attached to the lid of the chamber and a video camera was focused on the filter so that the filter could be seen on a screen in the control room. Prior to analysis, the proton beam was collimated to a size of approximately $7 \times 1 \mathrm{~mm}$ to match the size of each hourly PM sample, or spot. Using a computer program developed in-house, each spot was manually located and recorded in an electronic file that would later be used to automatically perform IBA measurements on each spot. Table A1 presents a sample program worksheet used for the analysis of Streaker filters. The number of steps is related to how many degrees the filter is rotated $\left(0.25^{\circ}\right.$ per one step).

Table A.1. Sample program worksheet for the automated analysis of Streaker filters

\begin{tabular}{|c|c|}
\hline Number of steps from origin & Sample identifier \\
\hline 92 & S225_01 \\
\hline 102 & S225_02 \\
\hline 123 & S225_03 \\
\hline 144 & S225_04 \\
\hline 165 & S225_05 \\
\hline 186 & S225_06 \\
\hline 207 & S225_07 \\
\hline 227 & S225_08 \\
\hline$\ldots$ & $\ldots$ \\
\hline
\end{tabular}

After the stepping program was created for a filter, each spot on that filter was analyzed automatically under a beam current of $23 \mathrm{nA}$ and received a total irradiation of $4 \mu \mathrm{C}$. The process of creating the stepping program and running the IBA was repeated for each of the exposed Streaker filters collected throughout this thesis. 